\title{
Modules de Hodge Polarisables
}

\author{
Par \\ Morihiko SAITo*,**
}

\section{Introduction}

Dans [7], Deligne a introduit la notion de complexe pur, et démontré la stabilité par image directe par morphismes propres (i.e. la version relative de la conjecture de Weil). Cette théorie, combinée avec celle de faisceaux pervers, a été ensuite développée par Beilinson-Bernstein-Deligne-Gabber[1]: ils ont démontré le théorème de décomposition, et la stabilité des faisceaux pervers purs par images directes intermédiaires (en particulier, la pureté des complexes d'intersection) [loc. cit].

D'après la philosophie de Deligne [6,7], on a conjecturé qu'il existerait des objets en car. 0 , correspondant aux complexes purs, cf. [2,3,4] etc. Le but de cet article est de donner une réponse positive: en utilisant la théorie de $\mathscr{D}$-Modules filtrés, la théorie de la filtration V $[17,22]$ et la théorie de faisceaux pervers, on définit la notion de Module de Hodge polarisable (cf. 5.1-2), qui doit correspondre à celle de faisceau pervers pur, et on démontre la stabilité par images directes (perverses) par un morphisme projectif avec un théorème de Lefschetz fort relatif et une polarisation induite sur les parties primitives (i.e. une théorie de Hodge-Lefschetz relative), cf. 5.3.1.

Soient $X$ une variété analytique complexe lisse, $\mathscr{D}_{X}$ le faisceau des opérateurs différentiels analytiques muni de la filtration $F$ par degré d'opérateur, et $\operatorname{MF}\left(\mathscr{D}_{X}\right)$ la catégorie des $\mathscr{D}_{X}$-Modules filtrés. On dit que $(M, F) \in M F\left(\mathscr{D}_{X}\right)$ est holonome, si $M$ est un $\mathscr{D}_{X}$-Module holonome $[16,18]$ et $G r^{F} M$ est un $G r^{F} \mathscr{D}_{X}$-Module cohérent (i.e. $F$ est une bonne filtration de $M$ [loc. cit]). Soit $M F_{h}\left(\mathscr{D}_{X}\right)$ la sous-catégorie pleine de $M F\left(\mathscr{D}_{X}\right)$ des $\mathscr{D}_{X}$-Modules filtrés holonomes. D'après Kashiwara [16] on a le foncteur

$$
D R: M F_{h}\left(\mathscr{D}_{X}\right) \rightarrow \operatorname{Perv}\left(\boldsymbol{C}_{X}\right)\left(\subset D_{c}^{b}\left(\boldsymbol{C}_{X}\right)\right)
$$

Reçu le 28 janvier, 1988.

* Institute for Advanced Study, Princeton, U.S.A.

RIMS Kyoto University, Kyoto 606, Japan.

** Supporté par Sloan Foundation. 
induit par $D R(M, F)=\left[M \rightarrow \Omega_{X}^{1} \otimes M \rightarrow \cdots \rightarrow \Omega_{X}^{n} \otimes M\right][n]$, où $n=\operatorname{dim} X$. Ici, pour $R$ un sous-corps de $C, \operatorname{Perv}\left(R_{X}\right)$ est la sous-catégorie pleine de $D_{c}^{b}\left(R_{X}\right)$ (la catégorie dérivée des $R_{X}$-Modules à cohomologies constructibles) définie par les conditions [1]:

$\operatorname{dim} \operatorname{supp} \mathcal{H}^{i} K \leqq-i, \quad \operatorname{dim} \operatorname{supp} \mathcal{H}^{i} D K \leqq-i$ pour $K \in D_{c}^{b}\left(R_{X}\right)$, où $D$ est le foncteur dual de Verdier [32]. D'après [1] $\operatorname{Perv}\left(R_{X}\right)$ est une catégorie abélienne, et $D R$ est exact et fidèle, car $D R$ induit le foncteur exact de catégories triangulées $D R: D_{h}^{b}\left(\mathscr{D}_{X}\right) \rightarrow D_{c}^{b}\left(C_{X}\right)$, et $M=0$ si $D R(M)=0$ pour un module holonome $\mathrm{M}$.

Soit $i: X \rightarrow Y$ une immersion fermée de variétés analytiques lisses. On a alors le foncteur image directe (cf. [2,21]):

$$
\int_{i}: M F_{h}\left(\mathscr{D}_{X}\right) \rightarrow M F_{h}\left(\mathscr{D}_{Y}\right)
$$

tel que $\int_{i}(M, F)=\left(\int_{i} M, F\right)$ avec $\int_{i} M=i_{*}\left(\mathscr{D}_{Y<X} \otimes \mathscr{D}_{X} M\right)$ (cf. [18]), où la filtration $F$ est la convolution de celle de $D_{Y \leftarrow X}=\Omega_{X}^{n} \otimes_{i^{-1} \mathcal{O}_{Y}} i^{-1}\left(\mathscr{D}_{Y} \otimes\left(\Omega_{Y}^{m}\right)^{-1}\right)$ et de $M$, i. e. si on choisit des coordonnées locales $\left(y_{1}, \cdots, y_{m}\right)$ de $Y$ telles que $X=\left\{y_{n+1}\right.$ $\left.=\cdots=y_{m}=0\right\}$, on a

$$
\int_{i} M=i_{*} M[\partial], \quad F_{p} \int_{i} M=\sum_{q+|\nu| \leq p-\gamma} i_{*} F_{q} M \otimes \partial^{v}
$$

où $\partial=\left(\partial_{n+1}, \cdots, \partial_{m}\right), \nu=\left(\nu_{n+1}, \cdots, \nu_{m}\right) \in N^{r}$ et $r=m-n=\operatorname{codim}_{Y} X$. On vérifie facilement $D R \circ \int_{i}=i_{*} \circ D R, c f$. $\S 2$. Ici on utilise les $\mathscr{D}$-Modules à gauche seulement dans cette introduction. La définition de l'image directe et la vérification de la compatibilité ci-dessus sont plus faciles dans le cas de $\mathscr{D}$-Modules à droite. Pour la transformation de $\mathscr{D}_{X}$-Modules à gauche dans ceux à droite on utilise le foncteur $\left(\Omega_{X}^{n}, F\right) \otimes \mathcal{O}_{X}$ avec $G r_{p}^{F} \Omega_{X}^{n}=0$ pour $p \neq-n$. Le décalage de la filtration $F$ de l'image directe vient de cette transformation.

On définit $\operatorname{MF}_{h}\left(\mathscr{D}_{X}, \boldsymbol{Q}\right)$ la catégorie des $\mathscr{D}_{X}$-Modules filtrés holonomes munis de $\boldsymbol{Q}$-structure par le produit de $M F_{h}\left(\mathscr{D}_{X}\right)$ et $\operatorname{Perv}\left(\boldsymbol{Q}_{X}\right)$ sur $\operatorname{Perv}\left(\boldsymbol{C}_{X}\right)$, i.e. les objets sont les couples d'objets $(M, F, K) \in M F_{h}\left(\mathscr{D}_{X}\right) \times \operatorname{Perv}\left(\boldsymbol{Q}_{X}\right)$ munis d'un isomorphisme $\alpha: D R(M, F) \cong C \otimes_{Q} K$ dans $\operatorname{Perv}\left(C_{X}\right)$, et les morphismes sont les couples de morphismes compatibles avec $\alpha$. Alors on a l'image directe

$$
i_{*}: M F_{h}\left(\mathscr{D}_{X}, \boldsymbol{Q}\right) \rightarrow M F_{h}\left(\mathscr{D}_{Y}, \boldsymbol{Q}\right)
$$

par $i_{*}(M, F, K)=\left(\int_{i}(M, F), i_{*} K\right)$ pour une immersion fermée $i$ comme cidessus. On définira $M H(X, w)$ la catégorie des Modules de Hodge de poids $w$ 
comme une sous-catégorie pleine de $M F_{h}\left(\mathscr{D}_{X}, \boldsymbol{Q}\right)$ en utilisant les foncteurs cycles évanescents.

Soit $g$ une fonction holomorphe sur $X$. On définit l'immersion par graphe $i_{g}: X \rightarrow X \times \boldsymbol{C}$ par $i_{g}(x)=(x, g(x))$. Posons $(\tilde{M}, F)=\int_{i_{g}}(M, F)$ pour $(M, F) \in$ $M F_{h}\left(\mathscr{D}_{X}\right)$. Si $M$ est régulier au sens de [18], on a la filtration $V$ sur $\tilde{M}$ dont les gradués associés calculent les cycles évanescents de $D R(M)$, cf. [17]. Si de plus la monodromie $T$ de $\psi_{g} D R(M)$ est quasi-unipotente, la filtration $V$ est indexée par $\boldsymbol{Q}$ et on a les isomorphismes canoniques

$$
D R\left(\oplus_{-1<\alpha \leqq 0} G r_{V}^{\alpha} \tilde{M}\right)=\psi_{g} D R(M)[-1], D R\left(G r_{V}^{-1} \tilde{M}\right)=\phi_{g, 1} D R(M)[-1]
$$

tels que $\exp \left(-2 \pi i t \partial_{t}\right)$ correspond à la monodromie $T$, cf. [loc. cit] (voir aussi 3.4.12), où $\phi_{g, 1}$ est la partie à monodromie unipotente de $\phi_{g}$ (cf. [10] pour la définition de cycles évanescents $\psi_{g}, \phi_{g}$ ). Ici $V$ est décroissante et $t \partial_{t}-\alpha$ est nilpotent sur $G r_{V}^{\alpha} \tilde{M}$, où $t$ est la coordonnée de $\boldsymbol{C}$. Si les conditions (cf. $(3.2 .1 .2-3))$ :

$$
\begin{array}{lll}
t: F_{p} V^{\alpha} \tilde{M} \stackrel{F_{p}}{\rightarrow} V^{\alpha+1} \tilde{M} & \text { pour } & \alpha>-1 \\
\partial_{t}: F_{p} G r_{V}^{\alpha} \tilde{M} \stackrel{\rightarrow}{\rightarrow} F_{p+1} G r_{V}^{\alpha-1} \tilde{M} & \text { pour } & \alpha<0
\end{array}
$$

sont satisfaites pour $(M, F, K) \in M F_{h}\left(\mathscr{D}_{X}, \boldsymbol{Q}\right)$, on définit les cycles évanescents de $(M, F, K)$ le long de $g$ par:

$$
\begin{aligned}
& \psi_{g}(M, F, K)=\left(\oplus_{-1<\alpha \leqq 0} G r_{V}^{\alpha}(\tilde{M}, F), \quad \psi_{g} K[-1]\right) \\
& \phi_{g, 1}(M, F, K)=\left(G r_{V}^{-1}(\tilde{M}, F[-1]), \quad \phi_{g, 1} K[-1]\right) .
\end{aligned}
$$

Ici on peut construire les isomorphismes dans (0.2) en utilisant la condition (0.3) et la condition $\operatorname{Gr}_{V}^{\alpha}(\tilde{M}, F) \in M F_{h}\left(\mathscr{D}_{X}\right)$ pour $\alpha>-1$, s'il existe la filtration $V$ indexée par $\boldsymbol{Q}$ et satisfaisant les conditions (3.1.1.1-3), cf. 3.4.12. Mais pour simplifier l'exposé, on suppose que $M$ soit régulier et que la monodromie $T$ soit quasi-unipotente, lorsqu'on dit que $(M, F, K)$ satisfait les conditions $(0.3-4)$, cf. 5.1.18.

On dit que $(M, F, K) \in M F_{h}\left(\mathscr{D}_{X}, \boldsymbol{Q}\right)$ est à support strict $Z$, si supp $M=Z$, $Z$ est irréductible et $M$ (ou $K$ ) n'a ni un sous-objet ni un objet quotient nontrivial dont le support est plus petit que $Z$. Soit $M F_{h}\left(\mathscr{D}_{X}, \boldsymbol{Q}\right)_{Z}$ la sous-catégorie pleine de $M F_{h}\left(\mathscr{D}_{X}, \boldsymbol{Q}\right)$ des objets à support strict $Z$ ou $\emptyset$. On dit que $(M, F, K)$ $\in M F_{h}\left(\mathscr{D}_{X}, \boldsymbol{Q}\right)$ est localement décomposable par support strict, si pour tout ouvert $U$ de $X$, la restriction de $(M, F, K)$ à $U$ est la somme directe de $\left(M_{Z}, F, K_{Z}\right) \in M F_{h}\left(\mathscr{D}_{U}, \boldsymbol{Q}\right)_{Z}$ pour $Z$ les sous-variétés fermées irréductibles de $U$, 
où $\left(M_{Z}, F, K_{Z}\right)$ sera dit la $Z$-composante de $\left.(M, F, K)\right|_{U}$. Cette propriété est de nature locale, car la décomposition est unique. Si $(M, F, K)$ satisfait les conditions (0.3-4), $(M, F, K)$ est localement décomposable par support strict, si on a

$$
G r_{V}^{-1}(\tilde{M}, F)=\operatorname{Im} \text { can } \oplus \operatorname{Ker} \operatorname{Var} \quad \text { dans } M F\left(\mathscr{D}_{U}\right)
$$

pour toute $g$ définie sur un ouvert $U$ de $X$, où can: $G r_{V}^{0}(M, F[1]) \rightarrow G r_{V}^{-1}(M, F)$ et $\operatorname{Var}: G r_{V}^{-1}(M, F) \rightarrow G r_{V}^{0}(M, F)$ sont définis par $-\partial_{t}$ et $t$, cf. 5.1.4-5.

Soit $M F_{h}\left(\mathscr{D}_{X}, \mathbb{Q}_{\text {ldss }}\right.$ la sous-catégorie pleine de $M F_{h}\left(\mathscr{D}_{X}, \mathbb{Q}\right)$ des objets satisfaisant les conditions (0.3-5) pour toute $g$ localement définie. Alors $M H(X, w)$ est la plus grande sous-catégorie pleine de $M F_{h}\left(\mathscr{D}_{X}, \mathscr{Q}_{l d s s}\right.$ satisfaisant les conditions (cf. 5.1.6):

(0.6) Si supp $(M, F, K)=\{x\}$, il existe $\left(H_{C}, F, H_{Q}\right)$ une $Q$-structure de Hodge de poids $w$ telle que $(M, F, K)=\left(i_{x}\right)_{*}\left(H_{C}, F, H_{Q}\right)$, où $i_{x}:\{x\} \rightarrow X$ et $F_{p}=$ $F^{-p}$ sur $H_{C}$ de sorte que $\left(H_{C}, F\right) \in M F_{h}\left(\mathscr{D}_{\{x\}}\right)$.

(0.7) Pour toute $g$ définie sur un ouvert $U$ de $X$ et pout toute sous-variété fermée irréductible $Z$ de $U$ telle que $g^{-1}(0) \nsupseteq Z$, on a $G r_{i-w+1}^{W} \psi_{g}$ $\left(M_{Z}, F, K_{Z}\right), G r_{i-w}^{W} \phi_{g, 1}\left(M_{Z}, F, K_{Z}\right) \in M H(U, i)$, où $\left(M_{Z}, F, K_{Z}\right)$ est la $Z$ composante de $\left.(M, F, K)\right|_{U}$.

Ici, $W$ est la filtration de monodromie, i.e. $N\left(W_{i}\right) \subset W_{i-2}$ et $N^{j}: G r_{j}^{W} \stackrel{\rightarrow}{\rightarrow} G r_{-j}^{W}$ $(j>0)$, cf. [7], où $N$ est définie par $-\left(t \partial_{t}-\alpha\right)$ sur $G r_{V}^{\alpha} \tilde{M}$ et par $(2 \pi i)^{-1} \otimes \log T_{u}$ sur $\psi_{g} K$ et $\phi_{g, 1} K$ avec $T=T_{u} T_{s}$ la décomposition de Jordan de la monodromie. Notons que ces conditions sont bien-définies par récurrence sur la dimension $\mathrm{du}$ support, et qu'elles sont locales. Posons $M H_{Z}(X, w)=M H(X, w) \cap M F_{h}$ $\left(\mathscr{D}_{X}, \mathbb{Q}\right)_{Z}$. On a alors la décomposition localement finie

$$
M H(X, w)=\bigoplus_{Z} M H_{Z}(X, w) .
$$

Une polarisation de $(M, F, K) \in M H(X, w)$ est un accouplement $S: K \otimes K \rightarrow$ $a_{X}^{\prime} Q(-w)\left(=Q_{X}(n-w)[2 n]\right)$ satisfaisant les conditions (cf. 5.2.10):

(0.8) Si supp $M=\{x\}$, il existe une polarisation $S^{\prime}$ de $\left(H_{C}, F, H_{Q}\right)$ au sens de [6] telle que $S=\left(i_{x}\right)_{*} S^{\prime}$, où $i_{x}$ et $\left(H_{C}, F, H_{Q}\right)$ sont comme dans la condition (0.6).

(0.9) $S$ est compatible avec la filtration de Hodge $F$, i.e. le morphisme $K \rightarrow$ $(\mathbb{D} K)(-w)$ correspondant à $S$ se prolonge en un morphisme $(M, F, K)$ $\rightarrow \mathbb{D}(M, F, K)(-w)$ de $M F_{h}\left(\mathscr{D}_{X}, Q\right)$.

(0.10) Pour toute $g$ et $Z$ comme dans (0.7), l'accouplement induit $G r^{W h} \psi_{g} S \circ$ 
$\left(i d \otimes N^{i}\right): P_{N} G r_{i}^{W} \psi_{g} K_{Z}[-1] \otimes P_{N} G r_{i}^{W} \psi_{g} K_{Z}[-1] \rightarrow a_{U}^{!} \boldsymbol{Q}(1-w-i)$ est une polarisation de la partie primitive $P_{N} G r_{i}^{W} \psi_{g}\left(M_{Z}, F, K_{Z}\right)$,

cf. 5.2.3 pour la définition de ${ }^{p} \psi_{g} S$, où les conditions sont encore par récurrence sur la dimension du support. Ici $\boldsymbol{D}(M, F, K)=(\boldsymbol{D}(M, F), \boldsymbol{D} K)$ est définie par la compatibilité du foncteur dual analytique et topologique, cf. 2.4 .12 et 2.4.15 (voir aussi $[18,23]$ ), et $\boldsymbol{D}(M, F)$ appartient à $M F_{h}\left(\mathscr{D}_{X}\right)$, i.e. la filtration $F$ de $\boldsymbol{D}(M, F)$ est strictement compatible à la différentielle du complexe, sii $G r^{F} M$ est un $G r^{F} \mathscr{D}_{X}$-Module de Cohen-Macaulay. Mais la dernière condition est satisfaite pour les Modules de Hodge, cf. 5.1.13. Noton qu'une polarisation est compatible à la décomposition par support strict, i.e. $S=\oplus S_{Z}$ avec $S_{Z}$ une polarisation de la $Z$-composante, $\operatorname{car} \operatorname{Hom}\left(K_{Z}, K_{Z^{\prime}}\right)=0$ pour $Z \neq Z^{\prime}$. On dit qu'un Module de Hodge est polarisable, s'il a une polarisation. On notera $M H(X, w)^{p}$ (resp. $\left.M H_{Z}(X, w)^{p}\right)$ la sous-catégorie pleine de $M H(X, w)$ (resp. $M H_{Z}$ $(X, w))$ des Modules de Hodge polarisables.

Soient $f: X \rightarrow Y$ un morphisme propre de variétés analytiques lisses, et $(M, F, K) \in M F_{h}\left(\mathscr{D}_{X}, \boldsymbol{Q}\right)$. Soient $i: X \rightarrow X \times Y$ la immersion par graphe de $f$, et $p: X \times Y \rightarrow Y$ la projection. On définit l'image directe $f_{*}(M, F, K)=\left(\int_{f}(M, F)\right.$, $\left.f_{*} K\right) \operatorname{par} \int_{f}(M, F)=\boldsymbol{R} p_{*} D R_{X \times Y / Y}(\tilde{M}, F)$ avec $(\tilde{M}, F)=\int_{i}(M, F)$ et

$$
F_{i} D R_{X \times Y / Y} \tilde{M}=\left[F_{i} \tilde{M} \rightarrow \Omega_{X}^{1} \otimes F_{i+1} \tilde{M} \rightarrow \cdots \rightarrow \Omega_{X}^{n} \otimes F_{i+n} \tilde{M}\right][n],
$$

cf. $[2,21]$ (voir aussi $[6,36]$ pour le cas $Y=p t$ ). On vérifie facilement $D R \circ \int_{f}=$ $f_{*} \circ D R$, cf. $\S 2$ pour la définition de l'image directe plus intrinsèque en utilisant les Modules induits. Si $\int_{f}(M, F)$ est strict, i.e. la différentielle du complexe est strictement compatible à la filtration $F$, on définit

$$
\mathscr{H}^{j} f_{*}(M, F, K)=\left(\mathcal{H}^{j} \int_{f}(M, F),{ }^{p} \mathcal{H}^{j} f_{*} K\right) \in M F_{h}\left(\mathscr{D}_{Y}, \boldsymbol{Q}\right)
$$

car $D R \circ \mathscr{H}^{j}={ }^{p} \mathcal{H}^{j} \circ D R$, où ${ }^{p} \mathscr{H}^{j}$ est le foncteur cohomologie perverse, cf. [1]. Le théorème principal de cet article est (cf. 5.3.1):

Théorème 1. Soient $f: X \rightarrow Y$ un morphisme projectif de variétés analytiques lisses, et $(M, F, K) \in M H(X, w)$ muni d'une polarisation $S$. Alors
i) $\int_{f}(M, F)$ est strict, et $\mathcal{H}^{j} f_{*}(M, F, K) \in M H(Y, w+j)^{p}$.
ii) $l^{j}: \mathscr{H}^{-j} f_{*}(M, F, K) \stackrel{\sim}{\rightarrow} \mathscr{H}^{j} f_{*}(M, F, K)(j)$ pour $j>0$.
iii) $(-1)^{j(j-i) / 2 p} \mathcal{H} f_{*} S \circ\left(i d \otimes l^{j}\right): P_{l}^{p} \mathscr{H}^{-j} f_{*} K \otimes P_{l}^{p} \mathscr{H}^{-j} f_{*} K \rightarrow a_{Y}^{!} \boldsymbol{Q}(j-w)$ est une polarisation de la partie l-primitive $P_{l} \mathscr{H}^{-j} f_{*}(M, F, K)$ pour $j \geq 0$. 
Dans ii), ( $j)$ est le twist de Tate pour $j \in \mathbb{Z}$, i.e. $(M, F)(j)=(M, F[j])$ avec $F[j]_{p}=F_{p-j}$, cf. (2.0.2), et $l$ est la première classe de Chern d'un faisceau inversible $f$-ample. Pour la démonstration du théorème 1 , on vérifie d'abord

Lemme 1. $(M, F, K) \in M H_{Z}(X, w)$ (resp. $\left.M H_{Z}(X, w)^{p}\right)$ est génériquement (sur Z) une variation de $\mathbf{Q}$-structure de Hodge (resp. Q-structure de Hodge polarisable) de poids $w$-dim $Z$.

En effet, cela résulte des deux lemmes suivants (cf. 5.1.8-10, 5.2.11-12):

Lemme 2. $M H_{Z}(X, w)$ (resp. $\left.M H_{Z}(X, w)^{p}\right)$ ne dépend que de $Z$, i.e. elle est indépendante de l'immersion de $Z$ dans une variété lisse $X$.

Lemme 3. $(M, F, K) \in M H_{X}(X, w)$ est une variation de Q-structures de Hodge de poids $w-\operatorname{dim} X$, si $K[-\operatorname{dim} X]$ est un système local sur $X$. De plus une polarisation de $(M, F, K)$ est une polarisation de la variation (au sens de [6]).

Ici on a utilisé aussi

Lemme 4. Pour $(M, F, K) \in M H_{Z}(X, w), K$ est un complexe d'intersection $g_{Z} L$ pour $L$ un système local défini sur un ouvert de Zariski lisse $U$ de $Z$.

La dernière assertion résulte immédiatement de la définition de support strict. Puisque les cycles évanescents d'un Module de Hodge sont 'mixtes' (i.e. Gr sont purs), il faut traiter un peu le cas mixte.

Une filtration $W$ de $(M, F, K) \in M F_{h}\left(\mathscr{D}_{X}, \boldsymbol{Q}\right)$ est un couple de filtrations sur $M$ et $K$ compatibles avec l'isomorphisme $\alpha$. Soit $M F_{h} W\left(\mathscr{D}_{X}, \mathbb{Q}\right)$ la catégorie des objets de $M F_{h}\left(\mathscr{D}_{X}, \mathbb{Q}\right)$ munis d'une filtration croissante $W$ localement finie sur $X$. Soit $M H W(X)$ (resp. $\left.M H W(X)^{p}\right)$ la sous-catégorie pleine de $M F_{h} W$ $\left(\mathscr{D}_{X}, Q\right)$ définie par la condition:

$$
G r_{i}^{W}(M, F, K) \in M H(X, i)\left(\text { resp. } M H(X, i)^{p}\right) \text { pour tout } i,
$$

i.e. les objets de $M H W(X)$ (resp. $\left.M H W(X)^{p}\right)$ sont les extensions (arbitraires) des objets de $M H(X, i)$ (resp. $\left.M H(X, i)^{p}\right)$, et ils sont appelés les Modules de Hodge $W$-filtrés (resp. Modules de Hodge polarisables $W$-filtrés). On démontre par récurrence sur la dimension du support (cf. 5.1.14, 5.2.13):

Lemme 5. $M H_{Z}(X, w), M H(X, w), M H W(X), M H_{Z}(X, w)^{p}, M H(X, w)^{p}$ et $M H W(X)^{p}$ sont des catégories abéliennes dont les morphismes sont strictement compatibles avec $F$ (et strictement bi-compatibles avec $F, W$ dans le cas de $M H W(X)$ et $M H W(X)^{p}, c f .1 .1 .15,1.2 .2,1.2 .6$ pour la définition de morphisme bi- (ou I-) strict), et $M_{Z}(X, w)^{p}$ et $M H(X, w)^{p}$ sont semi-simples. 
On définit le twist de Tate par

$$
(M, F, K ; W)(j)=(M, F[j], K(j) ; W[-2 j]), \text { cf. (2.0.2). }
$$

Soit $(M, F, K ; W)$ un Module de Hodge $W$-filtré muni d'un morphisme

$$
N:(M, F, K ; W) \rightarrow(M, F, K ; W)(-1)
$$

et un accouplement $S: K \otimes K \rightarrow a_{X}^{!} \boldsymbol{Q}(-w)=\boldsymbol{Q}_{X}(\operatorname{dim} X-w)[2 \operatorname{dim} X]$ tel que

$$
\begin{gathered}
S(i d \otimes N)+S(N \otimes i d)=0, \\
N^{j}: G r_{w+j}^{W}(M, F, K) \stackrel{\text { a }}{\rightarrow} G r_{w-j}^{W}(M, F, K)(-j) \quad \text { pour } \quad j>0,
\end{gathered}
$$

et que $G r^{W} S \circ\left(i d \otimes N^{j}\right): P_{N} G r_{w+j}^{W} K \otimes P_{N} G r_{w+j}^{W} K \rightarrow a_{X}^{1} \boldsymbol{Q}(-w-j)$ est une polarisation de la partie $N$-primitive $P_{N} G r_{w+j}^{W}(M, F, K)\left(:=\operatorname{Ker} N^{j+1}\right)$ pour $j \geqq 0$. On dit que $(M, F, K ; W)$ est fortement polarisé par $S, N$ avec poids $w$, si les conditions ci-dessus sont satisfaites. On démontre le théorème 1 et la proposition 1 ci-dessous par récurrence sur la dimension du support.

Proposition 1. Soient $f: X \rightarrow Y$ comme dans le théorème 1 , et $(M, F, K ; W)$ un Module de Hodge $W$-filtré fortement polarisé par $N, S$ avec poids и. Alors

i) $\int_{f}(M, F)$ est strict et $\left(\mathcal{H}^{j} f_{*}(M, F, K), W[j]\right) \in M H W(Y)^{p}$

ii) $l^{j}:\left(\mathscr{H}^{-j} f_{*}(M, F, K), W[-j]\right) \stackrel{\sim}{\rightarrow}\left(\mathscr{H}^{j} f_{*}(M, F, K), W[j]\right)(j)$ pour $j>0$.

iii) La partie l-primitive $\left(P_{l} \mathscr{H}^{-j} f_{*}(M, F, K), W[-j]\right)$ est fortement polarisée par $N,(-1)^{j(j-1) / 2}$ p $\mathcal{H} f_{*} S \circ\left(i d \otimes l^{j}\right)$ avec poids $w-j$ pour $j \geq 0$.

Ici $W \operatorname{sur} \int_{f} M, f_{*} K$ n'est pas strict; la filtration $W$ sur $\mathscr{H}^{j} \int_{f} M,{ }^{p} \mathscr{H}^{j} f_{*} K$ est définie par l'image de $\mathscr{H}^{j} \int_{f} W_{p} M$ dans $\mathscr{H}^{j} \int_{f} M$, etc.

D'après le lemme 1 et le théorème 1 , la preuve de la proposition 1 est réduite à un lemme de structure de Hodge bi-graduée polarisée (cf. 5.3.6) en utilisant la suite spectrale:

$$
E_{1}^{p q}=\mathcal{H}^{p+q} f_{*} G r_{-p}^{W}(M, F, K) \Rightarrow \mathcal{H}^{p+q} f_{*}(M, F, K)
$$

qui dégénère en $E_{2}$, cf. 5.3.5.

Pour la preuve du théorème 1 on peut supposer que $(M, F, K) \in M H_{Z}$ $(X, w)$. Si $\operatorname{dim} f(Z) \neq 0$, on prend toutes les fonctions $g$ localement définies sur $Y$ telles que $f^{-1} g^{-1}(0)$ ne contient pas $Z$. On applique la proposition 1 à $\left(\psi_{g f}(M, F, K), W\right),\left(\phi_{g f, 1}(M, F, K), W\right)$, et on utilise 3.3.17, cf. 5.3.4. Si dim $f(Z)=0$, on peut supposer que $X=\boldsymbol{P}^{m}, Y=p t$ d'après le lemme 2, et on prend un pinceau de Lefschetz en sorte que l'assertion est réduite au cas où $f$ se factorise par un morphisme plat $X \rightarrow \boldsymbol{P}^{1}$. Alors on applique le même argument 
que ci-dessus à $X \rightarrow \mathbb{P}^{1}$, et un résultat de Zucker [36] à $\mathbb{P}^{1} \rightarrow p t$ pour démontrer l'assertion i). Pour ii) et iii) on utilise le théorème de Lefschetz faible (cf. [1]; voir aussi 2.1.18) et aussi le pinceau de Lefschetz ci-dessus, cf. 5.3.8.

Pour appliquer le théorème 1 , il faut démontrer une réciproque du lemme 3 (cf. 5.4.3):

Théorème 2. Une variation de Q-structure de Hodge polarisée de poids $w$ sur une variété lisse $X$ est un Module de Hodge polarisé de poids $w+\operatorname{dim} X$.

Pour la démonstration il faut vérifier les conditions $(0.3-5),(0.7)$ et $(0.10)$ pour toutes les fonctions holomorphes localement définies sur $X$. D'après Hironaka [13] on a une désingularisation de $g$ (car $X$ est locale), et on se ramène au cas où $g^{-1}(0)$ est à croisements normaux (et à multiplicité constante) par le même argument que dans la preuve du théorème 1 dans le cas $\operatorname{dim} f(Z) \neq 0$. Alors on peut vérifier les assertions directement, où on utilise les trois filtrations compatibles (cf. 1.1.13) pour calculer les cycles évanescents, cf. 3.6.9.

Soit $Z$ une variété analytique irréductible. Alors on peut définir $M H_{Z}(w)$ (resp. $\left.M H_{z}(w)^{p}\right)$ la catégorie des Modules de Hodge (resp. Modules de Hodge polarisables) de poids $w$ à support strict $Z$ en utilisant les immersions locales de $X$ dans des variétés lisses d'après le lemme 2 (et 3.2.6), cf. 2.1.20, 5.3.12. On dit qu'une variation de structures de Hodge sur une variété lisse est géométrique, si elle est un facteur direct de l'image directe par un morphisme projectif lisse d'une variation de structures de Hodge dont le système local sous-jacent est constant de rang un, i.e. chaque fibre de la variation est le facteur direct de la cohomologie du fibre du morphisme au twist de Tate près. On dit qu'une variation géométrique sur un ouvert de Zariski lisse $U$ d'une variété analytique irréductible $Z$ est prolongeable rel. à $Z$, si le morphisme projectif lisse ci-dessus sur $U$ se prolonge en un morphisme projectif sur $Z$. On dit qu'une variation géométrique est algébriquement géométrique, si la variété et le morphisme projectif lisse ci-dessus sont algébrique. Notons qu'une variation algébriquement géométrique est prolongeable pour toutes les compactifications (partielles) algébriques d'après Nagata, Chow et Hironaka. Comme corollaire des théorèmes 1 et 2 , on obtient:

Corollaire 1. Si une variation de structures de Hodge géométrique définie sur un ouvert de Zariski lisse $U$ d'une variété analytique irréductible $Z$ est prolongeable rel. à $Z$, elle se prolonge canoniquement en un Module de Hodge polarisable a support strict $Z$. En particuler il existe uniquement un Module de Hodge polarisable à support strict $Z$ de poids $\operatorname{dim} Z$, dont le système local sous-jacent 
est le complexe d'intersection $\mathfrak{S C}_{Z} \mathbf{Q}$.

Alors le théorème 1 implique:

Corollaire 2. Pour une variété projective irréductible $Z$ et un système local $L$ sous-jacent à une variation de structures de Hodge algébriquement géométrique définie sur un ouvert de Zariski lisse de $Z$ (e.g. $\left.L=Q_{U}\right)$, il existe canoniquement une structure de Hodge pure polarisable sur la cohomologie d'intersection IH $^{*}$ $(Z, L:)=H^{\circ}\left(Z, g C_{Z} L\right)$

Corollaire 3. Soient $f: X \rightarrow Y$ un morphisme projectif de variétés analytiques irréductibles, et $L$ un systéme local sous-jacent à une variation de structures de Hodge géométrique sur un ouvert de Zariski de $X$. Si la variation géométrique est prolongeable rel à $X$, on a le théorème de décomposition de Beilinson-BernsteinDeligne-Gabber pour $f_{*} \mathcal{G} \mathcal{C}_{X} L$ l'image directe du complexe d'intersection à coèfficient $L$, i.e.

$$
\begin{gathered}
f_{*} \mathcal{G} \mathcal{C}_{X} L \cong \oplus_{j}\left({ }^{p} \mathcal{H}^{j} f_{*} \mathcal{G} \mathcal{C}_{X} L\right)[-j] \text { dans } D_{c}^{b}\left(\boldsymbol{Q}_{Y}\right), \\
{ }^{p} \mathcal{H}^{j} f_{*} \mathcal{G} \mathcal{C}_{X} L=\oplus_{Z^{\prime}} \mathcal{G} \mathcal{C}_{Z^{\prime}} L_{Z^{\prime}}^{l} \text { dans } \operatorname{Perv}\left(\boldsymbol{Q}_{Y}\right),
\end{gathered}
$$

où les $Z^{\prime}$ sont les sous-variétés fermées irréductibles de $Y$ et les $L_{Z^{\prime}}^{j}$ sont des systèmes locaux sur des ouverts de Zariski lisses de Z', cf. [1] pour le cas algébrique.

En effet, on a (0.11) d'après l'assertion ii) du théorème 1 et [8], et $(0.12)$ d'après l'assertion i) du théorème 1 et le lemme 4 . Notons que les systèmes locaux $L_{Z}^{j}$, sont semi-simples d'après [6] dans le cas algébrique.

Conjecture 1. Les corollaires ci-dessus restent valables (sans condition de prolongeabilité), si on remplace la condition "géométrique" par "polarisable".

Cette conjecture sera démontrée dans l'article suivant. En effet elle résulte de l'équivalence de catégories:

Conjecture 2. $M H_{Z}(w+\operatorname{dim} Z)^{p} \cong V S H_{\text {gén }}(Z, w)^{p}$.

Ici le terme à droite est la catégorie des variations de $\boldsymbol{Q}$-structures de Hodge polarisables sur des ouverts de Zariski lisses de $Z$ dont les monodromies locales sont quasi-unipotentes. Notons que la conjecture 2 est équivalente à la stabilité des Modules de Hodge polarisables par image directe intermédiaire par un morphisme ouvert tel que l'image directe du faisceau pervers sous-jacent par ce morphisme est à cohomologies constructibles.

La motivation originale de ce travail était de généraliser la démonstration 
analytique du théorème de décomposition (0.11-12) dans le cas $\operatorname{dim} Y=1$ et $\mathcal{I C}_{X} L=Q_{X}$, où $X, Y$ sont lisses, cf. [26, 27]. Notons que la proposition 4.2 .2 corrige la preuve de Steenbrink du théorème 5.9 dans [29], cf. 4.2.5. Par définition les Modules de Hodge polarisables sont stables par les foncteurs cycles évanescents gradués $G r^{W} \psi_{g}, G r^{W} \phi_{g, 1}$. Cette stabilité pour $\left(\mathcal{O}_{X}, F, \mathbb{Q}_{X}[\operatorname{dim} X]\right)$ est une généralisation d'un résultat de Varchenko [35] au cas singularités nonisolées, cf. [28, (4.1)]. Notons que la motivation originale de [26,27] était de donner une démonstration (et formule) naturelle du résultat de Varchenko.

Dans [2], Brylinski a proposé une autre définition de filtration de Hodge sur un $\mathscr{D}$-Module holonome régulier correspondant à un complexe d'intersection qui est génériquement sous-jacent à une variation de structures de Hodge polarisable, en utilisant l'ordre de Kashiwara-Kawai [18], cf. 5.4.9. Malheureusement il n'y a pas de méthode qui la relie à la théorie de Hodge classique sauf le cas où les singularité de la variation sont à croisements normaux, ni de stabilité de l'ordre de Kashiwara-Kawai par un morphisme projectif. Notons que, dans la théorie de Hodge, l'usage de $\mathscr{D}$-Modules filtrés remonte à Deligne: la généralisation d'un résultat de Griffiths sur l'intégral des formes rationnelles (i.e. la filtration de Hodge d'une variété ouverte est donnée par l'ordre de pôle de formes différentielles rationnelles, cf. [6, (3.1.11)]), car

$$
\mathbb{R} \Gamma\left(X,\left(j_{*}^{m} \Omega_{X^{*}}^{\circ}, F\right)\right)[\operatorname{dim} X]=\int_{X \rightarrow p t}\left(\mathcal{O}_{X}(* Y), F\right)
$$

par définition, où $X$ est une variété complexe lisse propre et $Y=X \backslash X^{*}$ est une hypersurface à croisements normaux. Une raison par laquelle $\mathscr{D}$-Modules filtrés sont essentiels, est qu'on obtient des complexes des $\mathscr{D}$-Modules filtrés avec une filtration stricte, et que ceci exprime des analogues relatifs de la dégénérescence de la suite spectrale: Hodge $\Rightarrow$ De Rham.

Dans cet article on n'utilise pas la notion de $\mathscr{D}$-Module holonome régulier; ceci pour répondre une question de Mebkhout. On peut, si on veut, supposer que tous les $\mathscr{D}$-Modules holonomes considérés dans cet article sont réguliers, car on peut démontrer que les conditions imposées aux Modules de Hodge entraînent leur régularité, cf. 5.1.18.

Le plan de cet article est comme suit:

En $\S 1$ on résout le problème de trois (ou plusieurs) filtrations, présenté dans [6]. On définit la notion de filtrations compatibles, celle de complexe (ou morphisme) strict, et on donne quelques critères, cf. 1.2.9, 1.2.12.

Pour deux sous-objets $A_{1}, A_{2}$ d'un objet $A$ d'une catégorie abélienne (e.g. la catégorie des espaces vectoriels), il existe toujours un diagramme carré de 
suites exactes courtes $C^{i, j}$ tel que $C^{-1,0}=A_{1}, C^{0,-1}=A_{2}$ et $C^{0,0}=A$ d'après le lemme des neuf (ou le lemme de Zassenhaus [loc. cit]). C'est pourquoi on n'a pas de problème pour deux filtrations. Mais pour trois sous-objets $A_{i}$ ( $j=$ $1,2,3$ ), un tel diagramme cubique n'existe plus en général (e.g. $A=\boldsymbol{R}^{2}, \operatorname{dim}$ $A_{j}=1$ et la position de $A_{j}$ est générique, i.e. $A_{i} \neq A_{j}$ pour $\left.i \neq j\right)$. On dit que trois filtrations $F_{j}(j=1,2,3)$ sont compatibles, si le diagramme cubique ci-dessus existe pour tous $F_{1}^{p}, F_{2}^{q}, F_{3}^{r}(p, q, r \in \mathbb{Z})$. Dans ce cas on peut vérifier que $G r_{F_{1}}^{p} G r_{F_{2}}^{q} G r_{F_{3}}^{r}$ ne dépend pas de l'ordre de filtrations. Les résultats principals de ce paragraphe sont les théorèmes 1.2.9 et 1.2.12 qui donnent des critères par récurrence pour que des filtrations soient compatibles et qu'un complexe multifiltré soit strict. En 1.3 on interprète les résultats de 1.1-2 du point de vue de la catégorie exacte au sens de Quillen [24]. On donne aussi un formalisme de la filtration décalée (cf. [6]) et de la filtration de monodromie (cf. [7]) dans la catégorie exacte. Ces résultats seront utilisés pour étudier le cas mixte dans l'article suivant.

En §2, on donne quelque formalisme de $\mathscr{D}$-Modules filtrés, i.e. la définition d'image directe et de dual, la dualité pour un morphisme propre avec la compatibilité de la dualité analytique et topologique par le foncteur $D R$. Le point clé est l'usage systématique de Modules induits $\operatorname{sur}(\mathcal{O}, \mathscr{D})$, i.e. de la forme $(L, F) \otimes \mathcal{O}(\mathscr{D}, F)$ pour un $\mathcal{O}$-Module filtré $(L, F)$, et l'introduction de complexes filtrés différentiels; ce qui simplifie considérablement les définitions ci-dessus et les vérifications des propriétés fonctorielles. Ici on utilise les $\mathscr{D}$ Modules à droite car c'est plus commode pour les images directes, pour la dualité et pour le foncteur $D R^{-1}$ qui associe un complexe de $\mathscr{D}$-Modules filtrés à un complexe filtré différentiel. Les lemmes clés sont 2.2.2 et 2.4.2.

En $\S 3$, on donne un formalisme de la filtration $V$ (comparer à [17] [22]). En 3.2 on introduit la notion de compatibilité entre la filtration de Hodge $F$ et la filtration $V$, et on démontre que la filtration $F$ est déterminée par sa restriction au complément d'un diviseur dans quelque cas, cf. 3.2.2-3. En 3.3 on définit l'image directe d'un $\mathscr{D}$-Module muni des filtrations $F$ et $V$ par un morphisme propre. On démontre que l'image directe est bi-strict au sens de $\S 1$, et que l'image directe de la filtration $V$ induit la filtration $V$ sur les cohomologies de l'image directe, si l'image directe de $G r_{a}^{V}(M, F)$ est strict pour tout $\alpha$, cf. 3.3.17. En 3.4 on construit un isomorphisme fonctoriel entre $D R G r^{V}$ et $\psi D R, \phi_{1} D R$, en généralisant la construction de Katz (voir [29]) et de Malgrange [22], où on utilise un résultat récent de Kashiwara-Schapira [19] (voir aussi [15]) pour démontrer la finitude de l'image directe par un morphisme 
non-propre associé à une fibration de Milnor, cf. 3.4.9. En 3.5, on définit les morphismes de restriction et de Gysin pour les $\mathscr{D}$-Modules filtrés holonomes dans le cas non-caractéristique de codimension un. On calcule aussi les cycles évanescents dans quelque cas; ceci est utilisé dans la preuve de 5.3.1, cf. 5.3.8. En 3.6 , on calcule les cycles évanescents $\psi_{g}(\mathcal{O}, F)$ dans le cas où $g^{-1}(0)$ est à croisements normaux et à multiplicité constante; ceci est utilisé dans la preuve de 5.4.3.

En $\S 4$, on démontre le lemme clé (cf. 4.2.2) pour le passage de $E_{1}$ à $E_{2}$ de la suite spectrale associée à $W$ (cf. 5.3 .5 et la proposition 1 dans cette introduction). Une nouvelle et simple démonstration de 4.2 .2 avec les signes exacts a été obtenue par Deligne, cf. [11].

En $\$ 5$, on définit les Modules de Hodge polarisables (cf. 5.1-2), et on démontre les théorèmes principals et leurs corollaires. Les points clés sont 5.1.14 (cf. lemme 5), 5.2.14 et 5.3.4 (cf. proposition 1 avec 3.3.17). On démontre aussi que $G r^{F} M$ est de Cohen-Macaulay pour $(M, F, K)$ un Module de Hodge, cf. 5.1.13, et on définit les accouplements ${ }^{p} \psi_{g} S,{ }^{p} \phi_{g, 1} S$ induits par le foncteur cycle évanescent, cf. 5.2.3. En 5.2.17-21, on donne un formalisme de suite spectrale associée à un objet quasi-filtré (ou spectral) qui a été peut-être connu par Verdier, cf. [1].

Je remercie P. Deligne, M. Kashiwara et B. Malgrange pour leurs mathématiques (et philosophies) qui m'ont introduit à ce sujet, et pour les conversations avec eux, qui m'ont donné une orientation correcte pour résoudre les difficultés techniques. Ce travail a été fait pendant mon séjour à l'Institut Fourier (CNRS), l'Université de Leiden (ZWO), l'IHES, ETH-Zürich, l'Université de Nice (CNRS), MPI Bonn et IAS Princeton. Je remercie les membres de ces instituts pour hospitalité.

Convention. On définit le décalage de filtration pour $n \in \mathbb{Z}$ par

$$
(F[n])^{i}=F^{i+n}, \quad(F[n])_{i}=F_{i-n}
$$

en sorte que ces définitions soient compatibles avec la convention usuelle: $F^{i}=F_{-i}$.

$\S 1$. I-filtrations compatibles et morphismes $I$-stricts

1.1. I-filtrations compatibles

1.2. Catégories dérivées et morphismes stricts

1.3. Complément

§2. DD-Modules filtrés 
2.1. $\mathscr{D}$-Modules filtrés et $\mathscr{B}$-Modules gradués

2.2. Foncteur de de Rham et complexes filtrés différentiels

2.3. Foncteur image directe

2.4. Foncteur dual

2.5. Dualité pour un morphisme propre

§3. Filtration de Malgrange-Kashiwara et cycles évanescents

3.1. Filtration rationnelle de Malgrange-Kashiwara

3.2. Compatibilité avec la filtration $F$

3.3. Catégories dérivées et images directes

3.4. Cycles évanescents

3.5. Le cas non-caractéristique

3.6. Le cas croisements normaux

\$4. Structures de Hodge graduées

4.1. Structures de Hodge graduées polarisées

4.2. Structures de Hodge bi-graduées polarisées

§5. Modules de Hodge polarisables

5.1. Modules de Hodge

5.2. Polarisation

5.3. Stabilité par image directe par un morphisme projectif

5.4. Variations de structures de Hodge polarisables

\section{§1. I-filtrations compatibles et morphismes $I$-stricts}

Soient $I$ un ensemble fini (non vide) et $\mathcal{A}$ une catégorie abélienne.

\subsection{I-filtrations compatibles}

1.1.1. Définition. Soit $C^{I}(\mathcal{A})$ la catégorie des complexes $I^{\text {uple }}$ naifs (cf. [9]). On dit que un objet $K$ de $C^{I}(\mathcal{A})$ est court si $K^{\nu}=0$ pour les éléments $\nu$ de $\boldsymbol{Z}^{I}$ tels que $\max _{i \in I}|\nu(i)|>1$, et exact si $K^{\nu-1_{i}} \stackrel{d_{i}}{\rightarrow} K^{\nu} \stackrel{d_{i}}{\rightarrow} K^{\nu+1_{i}}$ est exact pour tout $\nu$ de $\boldsymbol{Z}^{I}$ et pour tout $i$ de $I$.

On notera $C_{e c}^{I}(\mathcal{A})$ la sous-catégorie pleine de $C^{I}(\mathcal{A})$ des complexes exacts courts.

\subsubsection{Remarques.}

(1) $C_{e c}^{I}\left(\mathcal{A}^{\circ}\right)=C_{e c}^{I}(\mathcal{A})^{\circ}$

(2) $C_{e c}^{I}(\mathcal{A})$ est stable par extensions dans $C^{I}(\mathcal{A})$, donc c'est une catégorie exacte dans la catégorie abélienne $C^{I}(\mathcal{A})$. (Pour la définition de catégorie exacte, voir [21], [24].) 
1.1.3. Définition. Soit $2^{I}$ la catégorie des sous-ensembles de $I$, dont les morphismes sont les inclusions naturelles, soit $S_{I}(\mathscr{A})$ (resp. $\breve{S}_{I}(\mathcal{A})$ ) la catégorie des foncteurs contravariants (resp. covariants) de $2^{I}$ dans $\mathcal{A}$.

On définit un foncteur $S$ (resp. $\stackrel{\llcorner}{S})$ de $C^{I}(\mathcal{A})$ dans $S_{I}(\mathscr{A})$ (resp. $\stackrel{\vee}{S}_{I}(\mathscr{A})$ ) par

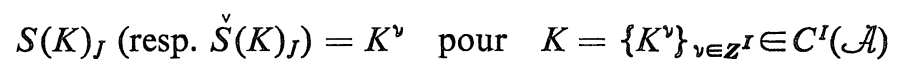

où $J \in 2^{I}$ et $\nu \in \mathbb{Z}^{I}$ satisfont la relation:

$$
\nu(i)=-1 \text { (resp. 1) si } i \in J \text {, et } 0 \text { sinon. }
$$

Soit $\stackrel{(v)}{S}{ }_{I}^{c}(\mathcal{A})$ l'image essentielle du foncteur $\stackrel{(v)}{S}: C_{e c}^{I}(\mathcal{A}) \rightarrow \stackrel{(v)}{S}(\mathcal{A})$, i.e. $\stackrel{(\sqrt[v]{ })}{S_{I}^{c}}(\mathcal{A})$ est la sous-catégorie pleine de $\stackrel{(v)}{S}(\mathcal{A})$ définie par:

$$
E \in \stackrel{(v)}{S}_{I}^{c}(\mathcal{A}) \text { sii } E \simeq \stackrel{(v)}{S}(K) \text { pour } K \in C_{e c}^{I}(\mathcal{A})
$$

Désormais le symbole ${ }^{(v)}$ signifie qu'ily a deux énoncés l'un pour $S$, $S_{I}^{c}(\mathcal{A})$, etc. et l'autre pour $\stackrel{\vee}{S}, \check{S}_{I}^{c}(\mathcal{A})$, etc.

Un objet de $S_{I}^{c}(\mathcal{A})\left(\operatorname{resp} . S_{I}^{c}(\mathcal{A})\right.$ ) sera dit un système compatible de sousobjets (resp. d'objets quotients).

1.1.4. Remarque. $S_{I}\left(\mathcal{A}^{\circ}\right)=\stackrel{\vee}{S}_{I}(\mathcal{A})^{\circ}, S_{I}^{c}\left(\mathcal{A}^{\circ}\right)=\check{S}_{I}^{c}(\mathcal{A})^{\circ}$.

1.1.5. Proposition. Le foncteur $\stackrel{(v)}{S}: C_{e c}^{I}(\mathcal{A}) \rightarrow \stackrel{(v)}{\rightarrow} S^{c}(\mathcal{A})$ est une équivalence de catégories; une suite

$$
0 \rightarrow E^{\prime} \rightarrow E \rightarrow E^{\prime \prime} \rightarrow 0
$$

dans $C_{e c}^{I}(\mathcal{A})$ est exacte dans la catégorie exacte $C_{e c}^{I}(\mathcal{A})$, sii l'image de (1.1.5.1) par $\stackrel{(v)}{S}$ est exacte dans la catégorie abélienne $\stackrel{(v)}{S_{I}}(\mathcal{A})$.

Preuve. On procède par récurrence sur $|I| . \quad$ Si $|I|=1$, l'assertion résulte du "lemme des 9 ". Si $|I|>1$, on a $I=I^{\prime} \cup\{*\}$ et la catégorie $S_{I}(\mathscr{A})$ est équivalente à la catégorie des morphismes de $S_{I^{\prime}}(\mathcal{A})$, donc, pour $E^{1}, E^{2} \in$ $C_{e c}^{I}(\mathcal{A})$, un morphisme $f: S\left(E^{1}\right) \rightarrow S\left(E^{2}\right)$ dans $S_{I}(\mathcal{A})$ correspond à un diagramme de $S_{I^{\prime}}^{c}(\mathcal{A})$ :

$$
\begin{aligned}
F_{-1}^{1} \stackrel{a_{1}}{\longrightarrow} & F_{0}^{1} \\
f_{-1} \downarrow & a_{2} \\
F_{-1}^{2} \stackrel{\downarrow}{\longrightarrow} & F_{0}^{2}
\end{aligned}
$$

où $S\left(E^{i}\right)$ correspond à $\left[a_{i}: F_{-1}^{i} \rightarrow F_{0}^{i}\right] \in \operatorname{Mor}\left(S_{I}^{c}(\mathcal{A})\right)$. Par hypothèse de récurrence, le diagramme (1.1.5.2) équivaut au diagramme de $C_{e c}^{I^{\prime}}(\mathcal{A})$ : 


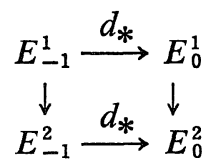

où $E_{j}^{i} \in C_{e c}^{I^{\prime}}(\mathcal{A})$ est défini par $\left(E_{j}^{i}\right)^{\mu}=\left(E^{i}\right)^{\nu}$ pour $\mu \in Z^{I^{\prime}}, \nu \in \boldsymbol{Z}^{I}$ tels que $\mu=$ $\left.\nu\right|_{I^{\prime}}, \nu(*)=j$. Puisque $d_{*}: E_{-1}^{i} \rightarrow E_{0}^{i}$ est strictement injectif par la définition de $F_{j}^{i}, E_{j}^{i}$, le diagramme (1.1.5.3) se prolonge en un diagramme:

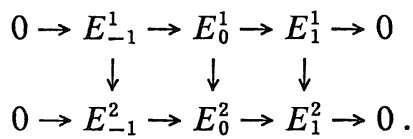

Un tel diagramme équivaut à se donner $g: E^{1} \rightarrow E^{2}$ tel que $S(g)=f$; d'où la première assertion. La deuxième assertion résulte du "lemme des 9". Même démonstration pour $\stackrel{S}{S}$.

1.1.6. Corollaire. $\stackrel{(v)}{S}(\mathcal{L}(\mathcal{A})$ est une catégorie exacte, dont une suite est exacte sii elle l'est dans $\stackrel{(\mathfrak{s})}{S_{I}}(\mathcal{A})$.

C'est clair.

1.1.7. Remarque. Pour $\phi \in \operatorname{Mor} C_{e c}^{I}(\mathcal{A}), \phi$ est un monomorphisme dans la catégorie abélienne $C^{I}(\mathcal{A})$ sii $\phi$ est un monomorphisme strict dans la catégorie exacte $C_{e c}^{I}(\mathcal{A})$. Mais $\phi \in$ Mor $S_{I}^{c}(\mathcal{A})$ n'est pas nécessairement un monomorphisme strict dans la catégorie exacte, même si c'est un monomorphisme dans la catégorie abélienne $S_{I}(\mathcal{A})$.

1.1.8. Définition. Soit $\phi$ un morphisme dans $\stackrel{(v)}{S}_{I}(\mathcal{A})$, alors on dit que $\phi$ est strict si Ker $\phi$ et Coker $\phi$ dans la catégorie abélienne $\stackrel{(v)}{S}_{I}(\mathcal{A})$ appartiennent à $\stackrel{(v)}{S_{I}^{c}}(\mathcal{A})$.

1.1.9. Proposition. Soit $\phi: E^{\prime} \rightarrow E$ un monomorphisme strict dans $S_{I}(\mathcal{A})$, i.e. on a une suite exacte dans la catégorie abélienne $S_{I}(\mathcal{A})$ :

$$
0 \rightarrow E^{\prime} \stackrel{\phi}{\rightarrow} E \rightarrow E^{\prime \prime} \rightarrow 0,
$$

telle que Coker $\phi=E^{\prime \prime} \in S_{I}^{c}(\mathcal{A})$, alors $E^{\prime} \in S_{I}^{c}(\mathcal{A})$ sii $E \in S_{I}^{c}(\mathcal{A})$.

Preuve. Par récurrence sur $|I|$. Si $|I|=1$, l'assertion résulte du lemme des cinq.

Si $|I|<1$, posons $I=I^{\prime} \cup\{*\}$, alors on a un diagramme

$$
\begin{gathered}
0 \rightarrow E_{0}^{\prime} \rightarrow E_{0} \rightarrow E_{0}^{\prime \prime} \rightarrow 0 \\
a^{\prime} \uparrow a \uparrow \quad a^{\prime \prime} \uparrow \\
0 \rightarrow E_{-1}^{\prime} \rightarrow E_{-1} \rightarrow E_{-1}^{\prime \prime} \rightarrow 0
\end{gathered}
$$


dans $S_{I^{\prime}}(\mathcal{A})$, qui correspond à (1.1.9.1). Si $E^{\prime}$ ou $E$ appartient à $S_{I}^{c}(\mathcal{A})$, on a $E_{i}^{\prime}, E_{i}, E_{i}^{\prime \prime} \in S_{I}^{c}(\mathcal{A})$ par hypothèse de récurrence; de plus, $a^{\prime}$ et $a^{\prime \prime}$ ou $a$ et $a^{\prime \prime}$ sont des monomorphismes stricts, donc $a^{\prime}$, $a$ et $a^{\prime \prime}$ sont des monomorphismes stricts par le lemme des cinq et d'après 1.1.5. L'assertion résulte du fait que $E \in S_{I}^{c}(\mathcal{A})$ sii $E_{-1}, E_{0} \in S_{I}^{c}(\mathcal{A})$ et $E_{-1} \rightarrow E_{0}$ est un monomorphisme strict (de même pour $E^{\prime}$ ).

1.1.10. Corollaire. $S_{I}^{c}(\mathcal{A})$ est stable par extensions dans $S_{I}(\mathcal{A})$.

1.1.11. Corollaire. Pour $\phi \in M o r S_{I}^{c}(\mathcal{A})$, $\phi$ est strict dans la catégorie exacte $S_{I}^{c}(\mathcal{A})$ sii il l'est dans $S_{I}(\mathcal{A})(c f .1 .1 .8)$.

1.1.12. Proposition. Soient $\phi: E \rightarrow F$ et $\psi: F \rightarrow G$ des morphismes dans $S_{I}(\mathcal{A})$ tels que $\psi$ soit un monomorphisme strict, alors $\phi$ est un monomorphisme strict sii $\psi \phi$ l'est.

Preuve. Si $\phi$ ou $\psi \phi$ est un monomoprhisme, $\phi, \psi$ et $\psi \phi$ sont des monomorphismes dans la catégorie abélienne, donc on applique la proposition 1.1.9 à la suite exacte:

$$
0 \rightarrow \text { Coker } \phi \rightarrow \text { Coker } \psi \phi \rightarrow \text { Coker } \psi \rightarrow 0 ;
$$

d'où l'assertion.

1.1.13. Définition. Soit $F_{I}(\mathcal{A})$ la catégorie additive des objet de $\mathcal{A}$ munis de $I$-filtrations, i.e. $O b F_{I}(\mathcal{A})=\left\{\left(A, F_{I}\right)\right\}$ où $A \in O b \mathcal{A}$ et $F_{I}=\left\{\left\{F_{i}^{p}\right\}_{p \in Z}\right\}_{i \in I}$ est une famille de filtrations décroissantes de $A$. Pour $\nu \in \mathbb{Z}^{I}$, on définit un foncteur

$$
F^{\nu}: F_{I}(\mathcal{A}) \rightarrow S_{I}(\mathcal{A}) \quad\left(\text { resp. } \stackrel{\vee}{F}^{\nu}: F_{I}(\mathcal{A}) \rightarrow \stackrel{\check{S}_{I}}{ }(\mathcal{A})\right)
$$

par

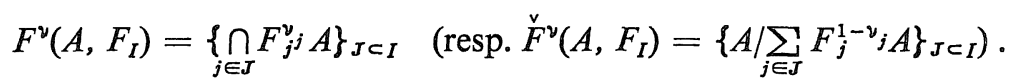

Soit $\stackrel{(\vee)}{F_{I}^{c}}(\mathcal{A})$ la sous-catégorie pleine de $F_{I}(\mathcal{A})$, définie par:

$$
\left(A, F_{I}\right) \in \stackrel{(v)}{F}{ }_{I}^{c}(\mathcal{A}) \operatorname{sii} \stackrel{(v)}{F}^{v}\left(A, F_{I}\right) \in \stackrel{(v)}{S}_{I}^{c}(\mathcal{A}) \text { pour tout } \nu \in \mathbb{Z}^{I} .
$$

Si $\left(A, F_{I}\right) \in F_{I}^{c}(\mathcal{A})$, on dit que $F_{I}$ sont des I-filtrations compatibles de $A$.

\subsubsection{Lemme.}

(1) $F_{I}^{c}(\mathcal{A})=\check{F}_{I}^{c}(\mathcal{A})\left(\operatorname{donc} F_{I}^{c}\left(\mathcal{A}^{\circ}\right)=F_{I}^{c}(\mathcal{A})^{\circ}\right)$

(2) Pour une suite

$$
0 \rightarrow E^{\prime} \rightarrow E \rightarrow E^{\prime \prime} \rightarrow 0
$$


de $F_{I}^{c}(\mathcal{A})$, l'image de (1.1.14.1) par $F^{\nu}$ est exacte sii celle de (1.1.14.1) par $\check{F}^{1-\nu}$ l'est. Pour un morphisme $\phi$ de $F_{I}^{c}(A), F^{\nu}(\phi)$ est strict sii $F^{1-\nu}(\phi)$ l'est

Ccla résulte de 1.1 .5 , car $S^{-1} F^{\nu}=S^{\nu}{ }^{\nu} F^{1-\nu} \operatorname{sur} F_{I}^{c}(\AA)$.

1.1.15. Définition. Une suite (1.1.14.1) de $F_{I}^{c}(\mathcal{A})$ est exacte, sii son image par $F^{\nu}$ l'est pour tout $\nu \in Z^{I}$. Un morphisme $\phi$ de $F_{I}^{c}(\mathcal{A})$ est strict, si $F^{\nu}(\phi)$ l'est pour tout $\nu \in \boldsymbol{Z}^{I}$.

1.1.16. Remarque. $F_{I}^{c}(\mathscr{A})$ est une catégorie exacte munie des suites exactes définies dans 1.1.15, cf. 1.3.3. Si un morphisme $\phi$ de $F_{I}^{c}(\mathcal{A})$ est strict, il existe $\operatorname{Ker} \phi$, Coker $\phi, \operatorname{Im} \phi$ et Coim $\phi$ dans $F_{I}^{c}(\mathcal{A})$ et Coim $\phi \Im \operatorname{Im} \phi$, car c'est valable pour tout $F^{\nu}(\phi)$ (ou $S^{-1} F^{\nu}(\phi)$ ).

\subsection{Catégories dérivées et morphismes stricts}

Soient $\stackrel{(v)}{S_{I}}(\mathcal{A}), \stackrel{(v)}{S_{I}^{c}}(\mathcal{A}), F_{I}(\mathcal{A})$ et $F_{I}^{c}(\mathcal{A})$ comme dans 1.1 .

1.2.1. Définition. Soient $C F_{I}(\mathcal{A}), C F_{I}^{c}(\mathcal{A})$ et $C S_{I}^{(v)}(\mathscr{A})$ respectivement la catégorie des complexes de $F_{I}(\mathcal{A}), F_{I}^{c}(\mathcal{A})$ et $\stackrel{(v)}{S}_{I}(\mathcal{A})$, soient $K F_{I}(\mathcal{A}), K F_{I}^{c}(\mathcal{A})$ et $K S_{I}(\mathcal{A})$ comme dans [31], i.e. les morphismes sont les classes d'équivalence homotope. Soit $H^{k}: C \stackrel{(v)}{S_{I}}(\mathcal{A}) \rightarrow \stackrel{(v)}{S_{I}}(\mathcal{A})\left(\operatorname{resp} . H^{k}: K S_{I}^{(v)}(\mathscr{A}) \rightarrow \stackrel{(v)}{S}_{I}(\mathcal{A})\right.$ ) le foncteur cohomologique usuel $(k \in Z)$. On notera encore $\stackrel{(v)}{F^{\nu}}: C F_{I}(\mathcal{A}) \rightarrow C S_{I}(\mathcal{A})$ (resp. $\left.\stackrel{(v)}{F^{\nu}}: K F_{I}(\mathcal{A}) \rightarrow K \stackrel{(v)}{S}_{I}(\mathcal{A})\right)$ le foncteur induit $\operatorname{par} \stackrel{(v)}{F^{\nu}}\left(\nu \in Z^{I}\right)$.

Soit $K F_{I}^{(v)}(\mathcal{A})^{\emptyset}$ (resp. $\left.K F_{I}^{c}(\stackrel{(}{\mathscr{A}})^{\emptyset}\right)$ la sous-catégorie pleine de $K F_{I}(\mathcal{A})$ (resp. $K F_{I}^{c}(\mathcal{A})$ ) définie par:

$$
\begin{aligned}
& E^{\cdot} \in K F_{I}^{(v)}(\mathcal{A})^{\emptyset}\left(\operatorname{resp} . K^{(v)} F_{I}^{c}(\mathcal{A})^{\emptyset}\right) \text { sii } H^{k} F^{\nu}\left(E^{*}\right)^{(v)}=0 \\
& \text { pour tout } \nu \in Z^{I}, k \in \boldsymbol{Z} .
\end{aligned}
$$

On définit

$$
\stackrel{(v)}{D F_{I}}(\mathcal{A})=K F_{I}(\mathcal{A}) / K F_{I}^{(v)}(\mathcal{A})^{\emptyset}\left(\operatorname{resp} . \stackrel{(v)}{(v)}_{I}^{c}(\mathcal{A})=K F_{I}^{c}(\mathcal{A}) / K F_{I}^{(v)}(\mathcal{A})^{\emptyset}\right)
$$

(Notons que $K F_{I}^{(v)}(\mathcal{A})^{\emptyset}$ (resp. $\left.K F_{I}^{c}(\mathcal{A})^{\emptyset}\right)$ est une sous-catégorie épaisse [31].)

Soit $C^{s} F_{I}^{(v)}(\mathcal{A})\left(\right.$ resp. $C^{s} F_{I}^{c}(\mathcal{A})$ ) la sous-catégorie pleine de $C F_{I}(\mathcal{A})$ (resp. $\left.C F_{I}^{c}(\mathcal{A})\right)$ définie par:

$$
\begin{aligned}
& E^{\cdot} \in C^{s^{(v)}} F_{I}(\mathcal{A})\left(\text { resp. } C^{s} F_{I}^{(v)}(\mathcal{A})\right) \text { sii } H^{k} F^{(v)}\left(E^{*}\right) \in \stackrel{(v)}{S^{c}}(\mathcal{A}) \\
& \text { pour tout } \nu, k \text {. }
\end{aligned}
$$

Similairement on définit la sous-catégorie pleine $D^{s(v)} F_{I}(\mathcal{A})\left(\operatorname{resp} . D^{s^{(v)}} F_{I}^{c}(\mathcal{A})\right)$ de $D F_{I}^{(v)}(\mathcal{A})$ (resp. $D F_{I}^{c}(\mathcal{A})$ ) en utilisant $H^{k} F^{(v)}$ (mais $D^{s} F_{I}^{(v)}(\mathcal{A})$ et $D^{s} F_{I}^{c}(\mathcal{A})$ ne sont pas des catégories triangulées en général.) 
1.2.2. Définition. On dit qu'un complexe $E^{\bullet}$ de $F_{I}(\mathscr{A})$ est strict (resp. costrict) si $E^{\bullet} \in C^{s} F_{I}(\mathcal{A})\left(\right.$ resp. $C^{s} F_{I}(\mathcal{A})$ ). Pour un morphisme $\phi$ dans $F_{I}(\mathcal{A})$, on dit que $\phi$ est strict (resp. costrict), si le complexe défini par $\phi$ l'est.

1.2.3. Proposition. Soient $E^{\bullet} \in C F_{I}^{c}(\mathcal{A})$ et $\nu \in \mathbb{Z}^{I}$, alors:

(i) $H^{k} F^{\nu}\left(E^{\circ}\right) \in S_{I}^{c}(\mathcal{A})$ pour tout $k$, sii $H^{k} \stackrel{v}{F}^{1-\nu}\left(E^{\circ}\right) \in \stackrel{v}{S}_{I}^{c}(\mathcal{A})$ pour tout $k$.

(ii) $H^{k} F^{\nu}\left(E^{\circ}\right)=0$ pour tout $k$, sii $H^{k} \stackrel{F}{1-\nu}^{1-\nu}\left(E^{\circ}\right)=0$ pour tout $k_{\text {。 }}$

(iii) $E^{\cdot} \in C^{s} F_{I}^{c}(\mathcal{A})$, sii $d^{k}: E^{k} \rightarrow E^{k+1}$ est un morphisme strict au sens de 1.1.15 pour tout $k$.

(iv) $H^{k} F^{\nu}\left(E^{\bullet}\right)=0$ pour tout $k, \nu$, sii $d^{k}: E^{k} \rightarrow E^{k+1}$ est un morphisme strict au sens de 1.1 .15 avec $\operatorname{Im} d^{k-1}=\operatorname{Ker} d^{k}$ pour tout $k$.

Il suffit de vérifier les assertion pour tout $S^{-1} F^{\nu} E^{\bullet}$, donc cette proposition résulte du lemme suivant:

1.2.4. Lemme. Soit $E^{\bullet}$ un complexe de $C_{e c}^{I}(\mathcal{A})$, et soit $d^{k}: E^{k} \rightarrow E^{k+1}$ la différentielle de $E^{\bullet}$. Posons

$$
\begin{aligned}
& Z^{k}=\operatorname{Ker} d^{k}, \quad B^{k}=\operatorname{Im} d^{k-1}, \quad C^{k}=\text { Coker } d^{k-1} \\
& H^{k}=Z^{k} / B^{k} \quad \text { dans } \quad C^{I}(\mathscr{A})
\end{aligned}
$$

alors les conditions suivantes sont équivalentes:
(a) $H^{k} \in C_{e c}^{I}(\mathcal{A}), \forall k$
(b) $B^{k} \in C_{e c}^{I}(\mathcal{A}), \forall k$
(c) $C^{k} \in C_{e c}^{I}(\mathcal{A}), \forall k$
(d) $Z^{k} \in C_{e c}^{I}(\mathcal{A}), \forall k$
(e) $S\left(H^{k}\right) \in S_{I}^{c}(\mathcal{A}), \forall k$

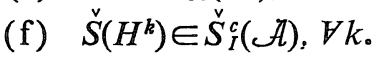

Preuve. On démontre l'équivalence de (a), (b), (d) et (e) par récurrence sur $|I|$; un argument dual montre celle de (a), (b), (c) et (f).

Si $|I|=1$, on a $H^{j}\left(Z^{k}\right)=0$ dour $j \neq 1$ par le lemme de serpent pour $d^{k}$ : $E^{k} \rightarrow E^{k+1}$, où $H^{j}: C^{I}(\mathcal{A}) \rightarrow \mathcal{A}$ est le foncteur cohomologique usuel. D'après la suite exacte

$$
0 \rightarrow Z^{k} \rightarrow E^{k} \rightarrow B^{k+1} \rightarrow 0
$$

on a $H^{j}\left(B^{k+1}\right)=H^{j+1}\left(Z^{k}\right)$, donc $H^{j}\left(B^{k}\right)=0$ pour $j \neq 0$. De la suite exacte

$$
0 \rightarrow B^{k} \rightarrow Z^{k} \rightarrow H^{k} \rightarrow 0,
$$

on déduit:

$$
H^{-1}\left(H^{k}\right)=H^{0}\left(B^{k}\right), \quad H^{0}\left(H^{k}\right)=0, \quad H^{1}\left(H^{k}\right)=H^{1}\left(Z^{k}\right)
$$

ce qui montre l'assertion pour $|I|=1$. 
Si $|I|>1$, posons $I=I^{\prime} \amalg\{*\}$, alors $E \in C_{e c}^{I}(\mathcal{A})$ est équivalent à une suite exacte courte de complexes de $C_{e c}^{I^{\prime}}(\mathcal{A})$

$$
0 \rightarrow E_{-1} \rightarrow E_{0} \rightarrow E_{1} \rightarrow 0 \text {. }
$$

Si une des conditions (a), (b), (d) et (e) est satisfaite, on a

$$
H^{k}\left(E_{i}\right) \cdot B^{k}\left(E_{i}\right), Z^{k}\left(E_{i}\right) \in C_{e c}^{I^{\prime}}(\mathcal{A}) \quad \text { pour } i=-1,0
$$

par hypothèse de récurrence; de plus, dans le cas où la condition (e) est satisfaite, $H^{k}\left(E_{-1}\right) \rightarrow H^{k}\left(E_{0}\right)$ est un monomosphisme strict dans $C_{e c}^{I^{\prime}}(\mathcal{A})$ (par la définition de $S_{I}^{c}(\mathcal{A})$ ), donc on peut appliquer l'assertion pour $|I|=1$ aux complexes de suites exactes courtes de $A$ :

$$
0 \rightarrow E_{-1}^{\nu} \rightarrow E_{0}^{\nu} \rightarrow E_{1}^{\nu} \rightarrow 0, \quad \nu \in Z^{I^{\prime}} ;
$$

d'où l'assertion.

1.2.5. Corollaire. $D F_{I}^{c}(\mathcal{A})=D \check{F}_{I}^{c}(\mathcal{A}), \quad C^{s} F_{I}^{c}(\mathcal{A})=C^{s} \check{F}_{I}^{c}(\mathcal{A})$,

$$
D^{s} F_{I}^{c}(\mathscr{A})=D^{s} \stackrel{\vee}{F}_{I}^{c}(\mathcal{A}) \text {. }
$$

1.2.6. Corollaire. Soit $\phi$ un morphisme de $F_{I}^{c}(\mathcal{A})$, alors les conditions suivantes sont equivalentes:

(a) $\phi$ est strict dans $F_{I}(\mathcal{A})$ (cf. 1.2.2),

(b) $\phi$ est costrict dans $F_{I}(\mathcal{A})$,

(c) $\phi$ est strict au sens de 1.1.15.

1.2.7. Définition. Pour $i \in I$, posons $I^{\prime}=I \backslash\{i\}$. Soient $p, q$ des entiers ou $\pm \infty$ tels que $p \leqslant q$; on définit un foncteur

$$
G_{I^{\prime}}^{p q}: F_{I}(\AA) \rightarrow F_{I^{\prime}}(\mathcal{A})
$$

par

$$
G_{I^{\prime}}^{p q}\left(A, F_{I}\right)=\left(F_{i}^{p} A / F_{i}^{q} A, F_{I^{\prime}}\right),
$$

où $F_{i}^{\infty} A=0, F_{i}^{-\infty} A=A$ et $F_{I^{\prime}}=\left\{F_{j}\right\}_{j \in I^{\prime}}$ est une famille des filtrations induites sur $F_{i}^{p} A / F_{i}^{q} A$. On a alors des morphismes naturels de foncteurs $G_{I^{\prime}}^{p q} \rightarrow G_{I^{\prime}}^{p^{\prime} q^{\prime}}$ pour $p \geqslant p^{\prime}, q \geqslant q^{\prime}$. Posons $G r_{F_{i}}^{p}=G_{I^{\prime}}^{p, p+1}, G_{I^{\prime}}^{p}=G_{I^{\prime}}^{p,+\infty}$ et $G_{I^{\prime}}=G_{I^{\prime}}^{-\infty,+\infty}$

1.2.8. Définition. Soit $\phi: E^{\prime} \rightarrow E$ un morphisme de $C F_{I}(\mathcal{A})$, alors on dit que $\phi$ est un monomorphisme strict cohomologique (m.s.c.), si $\phi: H^{k} F^{\nu} E^{\prime} \rightarrow H^{k} F^{\nu} E$ est un monomorphisme strict dans $S_{I}(\mathcal{A})$ pour tout $k, \nu$, cf. 1.1.8.

1.2.9. Théorème. Soit $E^{\bullet}=\left(A^{\bullet}, F_{I}\right) \in C F_{I}(\mathcal{A})$, soient $i \in I, I^{\prime}=I \backslash\{i\}$. Considérons les conditions suivantes: 
(0) $E^{\bullet} \in C^{s} F_{I}(\mathcal{A})$,

(i) $G_{I^{\prime}}^{p}\left(E^{\circ}\right) \in C^{s} F_{I^{\prime}}(\mathcal{A})$ pour tout $p \in \mathbb{Z}$,

(ii) $G_{I^{\prime}}\left(E^{\circ}\right) \in C^{s} F_{I^{\prime}}(\mathscr{A})$,

(iii) $G_{I^{\prime}}^{p}\left(E^{\circ}\right) \rightarrow G_{I^{\prime}}\left(E^{*}\right)$ est un m.s.c. pour tout $p \in \mathbb{Z}$,

(iv) $G_{I^{\prime}}^{p}\left(E^{\circ}\right) \rightarrow G_{I^{\prime}}^{q}\left(E^{\circ}\right)$ est un m.s.c. pour tout $p, q \in \mathbb{Z}$ tels que $p \geqslant q$,

(v) $G_{I^{\prime}}^{p+1}\left(E^{*}\right) \rightarrow G_{I^{\prime}}^{p}\left(E^{\circ}\right)$ est un m.s.c. pour tout $p \in \mathbb{Z}$;

alors:

(a) on $a$ : (0) $\Leftrightarrow$ (i) + (iii) $\Leftrightarrow$ (ii) + (iii) $\Rightarrow$ (iv) $\Leftrightarrow$ (v)

(b) si la condition:

(I) les limites inductives filtrantes existent dans $\mathcal{A}$ et sont exactes, est satisfaite, et si $\bigcup_{p} F_{i}^{p} A^{\circ}=A^{\circ}$, on $a$ :

$$
\text { (iii) } \Leftrightarrow \text { (iv) } \Leftrightarrow \text { (v) }
$$

(c) si la condition (I) et les conditions $\bigcup_{p} F_{i}^{p} A^{\circ}=A^{*} F_{i}^{p} A^{\circ}=0(p \gg 0)$ sont satisfaites, alors $(0) \Leftrightarrow(\mathrm{v})$

(d) si le morphisme $F^{v} G_{I^{\prime}}^{p} A^{k} \rightarrow F^{v} G r_{F_{i}}^{p} A^{k}$ est un épimprohisme dans $S_{I^{\prime}}(\mathcal{A})$ pour tout $\nu, p, k$, et si $F_{i}^{p} A^{\bullet}=0$ pour $p \gg 0$, alors la condition (v) est équivalente à la condition:

(vi) $\left(F_{i}^{p} A^{0}, F_{i}\right)$ est strict et $G r_{F_{i}}^{p}\left(E^{\circ}\right) \in C^{s} F_{I^{\prime}}(\mathcal{A})$ pour tout $p$.

Preuve. (a) résulte de 1.1 .9 et 1.1 .12 , (b) et (c) sont évidentes; prouvons maintenant $(\mathrm{d})$. Par hypothèse on a des suites exactes:

$$
0 \rightarrow F^{\nu} G_{I^{\prime}}^{p+1} A^{0} \rightarrow F^{\nu} G_{I^{\prime}}^{p} A^{\bullet} \rightarrow F^{\nu} G r_{F_{i}}^{p} A^{\bullet} \rightarrow 0 .
$$

Si (v) est satisfaite, on obtient des suites exactes:

$$
0 \rightarrow H^{k} F^{\nu} G_{I^{\prime}}^{p+1} A^{\bullet} \rightarrow H^{k} F^{\nu} G_{I^{\prime}}^{p} A^{\bullet} \rightarrow H^{k} F^{\nu} G r_{F_{i}}^{p} A^{\bullet} \rightarrow 0
$$

donc $G r_{F_{i}}^{p} E^{\bullet} \in C^{s} F_{I^{\prime}}(\mathcal{A})$ et $H^{k} F_{i}^{p+1} A^{0} \rightarrow H^{k} F_{i}^{p} A^{0}$ est un monomorphisme, i.e. $\left(F_{i}^{p} A^{0}, F_{i}\right) \in C^{s} F_{\{i\}}(\mathcal{A})$. Si (vi) est satisfaite, on vérifie par récurrence sur $p$ que (1.2.9.1) est exacte et que $H^{k} F^{\nu} G_{I^{\prime}}^{p} A^{\bullet} \in S_{I^{\prime}}^{c}(\mathcal{A})$; en effet, d'après 1.1.9, il suffit de montrer que $H^{k} F^{\nu} G_{I^{\prime}}^{p+1} A^{\bullet} \rightarrow H^{k} F^{\nu} G_{I^{\prime}}^{p} A^{\circ}$ est un monomorphisme dans $S_{I^{\prime}}(\mathcal{A})$, mais cela résulte de l'injectivité de $H^{k} F_{i}^{p+1} A \rightarrow H^{k} F_{i}^{p} A$ et de $H^{k} F^{\nu} G_{I^{\prime}}^{p+1} A \in S_{I^{\prime}}^{c}(\mathcal{A})$; d'où 1'assertion.

1.2.10. Corollaire. Soient $E^{\circ}=\left(A^{\circ}, F_{I}\right) \in C F_{I}^{c}(\AA)$ et $I=I^{\prime} \Perp\{i\}$. Si la condition (I) et les conditions $\bigcup_{p} F_{i}^{p} A=A, F_{i}^{p} A=0(p \gg 0)$ sont satisfaites, alors on a $E^{\bullet} \in C^{s} F_{I}^{c}(\mathcal{A})$, sii $\left(A^{\circ}, F_{i}\right)$ est strict et $G r_{F_{i}}^{p}\left(E^{\circ}\right) \in C^{s} F_{I}^{c}(\mathcal{A})$ pour tout $p$. 
En effet, l'hypothèse dans 1.2 .9 (d) est satisfaite d'après $E^{*} \in C F_{I}^{c}(\mathcal{A})$, cf. 1.2.11(2).

\subsubsection{Remarques.}

(1) On a $F_{I}^{c}(\mathcal{A})=F_{I}(\mathcal{A})$ si $|I| \leqslant 2$.

(2) On a $\left(F_{i}^{p} A^{\bullet}, F_{I}\right) \in C^{s} F_{I}(\mathcal{A})$ si $\left(A^{*}, F_{I}\right) \in C^{s} F_{I}(\mathcal{A})$; en particulier $\left(F_{i}^{p} A^{*}, F_{I}\right)$ $\in C F_{I}^{c}(\mathcal{A})$ si $\left(A^{\circ}, F_{I}\right) \in C F_{I}^{c}(\mathcal{A})$.

1.2.12. Théorème. Soient $E=\left(A, F_{I}\right) \in F_{I}(\mathcal{A})$ et $I=I^{\prime} \cup\{i\}$. Considérons les conditions suivantes:

(0) $E \in F_{I}^{c}(\mathcal{A})$

(i) $G_{I^{\prime}}^{p}(E) \in F_{I^{\prime}}^{c}(\mathcal{A})$ pour tout $p \in Z$

(ii) $G_{I^{\prime}}(E) \in F_{I^{\prime}}^{c}(\mathcal{A})$

(iii) $G_{I^{\prime}}^{p}(E) \rightarrow G_{I^{\prime}}(E)$ est strict pour tout $p \in \boldsymbol{Z}$

(iv) $G_{I^{\prime}}^{p}(E) \rightarrow G_{I^{\prime}}^{q}(E)$ est strict pour tout $p \geqslant q$

(v) $G_{I^{\prime}}^{p+1}(E) \rightarrow G_{I^{\prime}}^{p}(E)$ est strict pour tout $p \in \mathbb{Z}$,

(vi) $F^{\nu} G_{i^{\prime}}^{p}(E) \rightarrow F^{\nu} G r_{F_{i}}^{p}(E)$ est un épimorphisme dans $S_{I^{\prime}}(\mathcal{A})$ pour tout $p$, et $\operatorname{Gr}_{F}^{p}(E) \in F_{I}^{c},(\mathcal{A})$ pour tout $p \in Z$;

alors:

(a) on $a(0) \Leftrightarrow($ i $)+$ (iii) $\Leftrightarrow$ (ii) + (iii) $\Rightarrow$ (iv) $\Leftrightarrow(\mathrm{v}) \Leftrightarrow$ (vi)

(b) si la condition (I) et la condition $\bigcup_{p} F_{i}^{p} A=A$ sont satisfaites, on a (iii) $\Leftrightarrow$ (iv) $\Leftrightarrow(\mathrm{v}) \Leftrightarrow$ (vi)

(c) si la condition (I) et les conditions $\bigcup_{p} F_{i}^{p} A=A, F_{i}^{p} A=0(p \gg 0)$ sont satisfaites, on $a(0) \Leftrightarrow(\mathrm{vi})$.

Preuve. C'est un cas particulier du théorème 1.2.9 sauf $(\mathrm{v}) \Leftrightarrow(\mathrm{vi})$, mais cela résulte de la définition 1.2.2.

1.2.13. Corollaire. Soit $\left(A^{\bullet}, F_{I}\right) \in C^{s} F_{I}^{c}(\mathcal{A})$, on a alors $\left(F_{i}^{p} A^{*}, F_{I}\right) \in$ $C^{s} F_{I}^{c}(\mathcal{A})$ pour tout $i, p \in Z$, où $F_{I}$ est la famille de filtrations induites sur $F_{i}^{p} A$; donc les foncteurs $G r_{F}^{p_{i}}(i \in I), H^{j}$ commutent pour tout $p_{i}, j \in \boldsymbol{Z}$; en particulier, $\operatorname{pour}\left(A, F_{I}\right) \in F_{I}^{c}(\mathcal{A})$,

$$
G r_{F_{i}}^{p_{i}} G r_{F_{j}}^{p_{j}} \cdots G r_{F_{k}}^{p_{k}} A
$$

ne dépend pas de choix d'ordre de I.

Cela résulte de $1.2 .3,1.2 .9$ et 1.2.12.

1.2.14. Corollaire. Soient $I=\{i, j, k\}$ et $E=\left(A, F_{I}\right) \in F_{I}(\mathcal{A})$. Si la condition (I) et la condition $\bigcup_{p} F_{i}^{p} A=A$ sont satisfaites, alors $E \in F_{I}^{c}(\mathcal{A})$ sii $F_{i}^{p} F_{j}^{q} F_{k}^{r} A \rightarrow F_{j}^{q} F_{k}^{r} G r_{F_{i}}^{p} A$ est surjectif pour tout $p, q, r$. 
1.2.15. Remarque. Soient $A_{i}(i \in I)$ des sous-objets de $A \in \mathcal{A}$. On dit que les $A_{i}$ sont compatibles, s'il existe $S \in S_{I}^{c}(\mathcal{A})$ tel que $S_{\emptyset}=A$ et $S_{\{i\}}=A_{i}$. Pour des sous-objets $A_{i}(i \in I)$ de $A$, on définit des filtrations $F_{i} \operatorname{par} F_{i}^{-1} A=A$, $F_{i}^{0} A=A_{i}$ et $F_{i}^{1} A=0$. Alors les $A_{i}$ sont des sous-objets compatibles de $A$, sii $\left(A, F_{I}\right) \in F_{I}^{c}(\mathcal{A})$ par définition.

Pour $\left(A, F_{I}\right) \in F_{I}^{c}(\mathcal{A})$ et $j \in I, p \in \mathbb{Z}$, on définit une filtration $F_{*}$ de $A$ par $F_{*}^{-1} A=A, F_{*}^{0} A=F_{j}^{p} A$ et $F_{*}^{1} A=0$. Posons $\tilde{I}=I \Perp\{*\}$. Alors

$$
\left(A, F_{\tilde{I}}\right) \in F_{\tilde{I}}^{c}(\mathcal{A})
$$

d'après 1.2.12 en utilisant les suites exactes dans $F_{I^{\prime}}^{c}(\mathcal{A})$ (où $I^{\prime}=I \backslash\{i\}$ ):

$$
0 \rightarrow G_{I^{\prime}}^{q p} \rightarrow G_{I^{\prime}}^{r p} \rightarrow G_{I^{\prime}}^{r q} \rightarrow 0 \quad \text { pour } p>q>r, \text { cf. 1.2.9. }
$$

Pour $\left(A, F_{I}\right) \in F_{I}^{c}(\mathcal{A})$ et $j, k \in I, p, q \in Z$, on définit $F_{*}$ par $F_{*}^{0} A=F_{j}^{p} A \cap$ $F_{k}^{q} A$ ou $F_{j}^{p} A+F_{k}^{q} A$ avec $F_{*}^{-1} A=A$ et $F_{*}^{1} A=0$. Alors

$$
\left(A, F_{\tilde{I}}\right) \in F_{\tilde{I}}^{c}(\mathscr{A})
$$

d'après 1.2.12 et la stabilité des mono- (resp. épi-) morphismes stricts par compositions. On en déduit que pour $\left(A, F_{I}\right) \in F_{I}^{c}(\mathcal{A})$ tous éléments (de nombre fini) du sous-lattis du lattis des sous-objets de $A$, engendré par $F_{i}^{p} A$ pour tout $i, p$ sont des sous-objets compatibles de $A$; en particulier, le sous-lattis ci-dessus est distributif. La dernière assertion avec sa réciproque a été démontrée par Deligne en utilisant la théorie de lattis.

\subsection{Complément}

1.3.1. Soit $\mathcal{C}$ une catégorie exacte, alors une filtration (décroissante) d'un objet $E$ de $\mathcal{C}$ est une famille de monomorphismes stricts

$$
u_{i j}: F^{j} E \rightarrow F^{i} E \quad(i, j \in \mathbb{Z} \cup\{ \pm \infty\}, i<j)
$$

telle que $u_{i k}=u_{i j} u_{j k}$ pour $i<j<k$, où $F^{-\infty} E=E, F^{\infty} E=0$.

Soit $F(C)$ la catégorie additive d'objets de $\mathcal{C}$, munis d'une filtration décroissante, où les morphismes sont des familles de morphismes

$$
a_{i}: F^{i} E \rightarrow F^{i} E^{\prime} \quad(i \in \mathbb{Z} \cup\{ \pm \infty\})
$$

compatibles avec $\left\{u_{i j}\right\}$. On dit qu'une suite courte dans $F(\mathcal{C})$

$$
0 \rightarrow\left(E^{\prime}, F\right) \rightarrow(E, F) \rightarrow\left(E^{\prime \prime}, F\right) \rightarrow 0
$$

est exacte, si 


$$
0 \rightarrow F^{i} E^{\prime} \rightarrow F^{i} E \rightarrow F^{i} E^{\prime \prime} \rightarrow 0
$$

l'est pour tout $i \in \boldsymbol{Z} \cup\{ \pm \infty\}$. Notons que cette condition entraîne que

$$
\left.0 \rightarrow\left(F^{i} / F^{j}\right)\right) E^{\prime} \rightarrow\left(F^{i} / F^{j}\right) E \rightarrow\left(F^{i} / F^{j}\right) E^{\prime \prime} \rightarrow 0
$$

est exacte pour $-\infty \leqslant i<j \leqslant \infty$, où $\left(F^{i} / F^{j}\right) E=\operatorname{Coker} u_{i j}$ (cf. 1.3.2).

1.3.2. Soit $\tilde{\mathcal{C}}$ une catégorie abélienne telle que $\mathcal{C}$ soit une sous-catégorie pleine additive de $\tilde{\mathcal{C}}$, stable par extensions, et que'une suite courte de $\tilde{\mathcal{C}}$ soit exacte sii elle l'est dans $\tilde{\mathcal{C}}$ [21], [24].

Soit $\tilde{F}(\tilde{\mathcal{C}})$ la catégorie abélienne des foncteurs contravariants de $\boldsymbol{Z} \cup\{-\infty\}$ dans $\tilde{\mathcal{C}}$, i.e. les objets de $\tilde{F}(\tilde{\mathcal{C}})$ sont des familles de morphismes de $\tilde{\mathcal{C}}$ :

$$
\left\{u_{i j}: E^{j} \rightarrow E^{i} \quad(-\infty \leqslant i<j<\infty)\right\}
$$

tels que $u_{i j} u_{j k}=u_{i k}$ pour $i<j<k$; alors on peut identifier $F(\mathcal{C})$ avec une souscatégorie pleine de $\tilde{F}(\tilde{\mathcal{C}})$, de sorte que

$$
\begin{aligned}
& \left\{u_{i j}: E^{j} \rightarrow E^{i}\right\} \in F(\mathcal{C}) \text { sii } E^{j} \in \mathcal{C}(j \in \boldsymbol{Z} \cup\{-\infty\}) \\
& \text { et } u_{i j} \text { sont des monomorphismes stricts dans } \mathcal{C}(i<j) .
\end{aligned}
$$

On vérifie que $F(\mathcal{C})$ est stable par extensions dans $\tilde{F}(\tilde{C})$ et que une suite courte de $F(\mathcal{C})$ soit exacte sii elle l'est dans $\tilde{F}(\tilde{\mathcal{C}})$; en particulier, $F(\mathcal{C})$ est une catégorie exacte munie des suites exactes définies dans 1.3.1, de plus l'exactitude de (1.3.1.2) pour tout $i$ entraîne celle de (1.3.1.3) pour tout $i<j$.

1.3.3. Soit $I$ un ensemble fini (non vide). Supposons que $I$ soit totalement ordonné. On définit $F_{I}^{c}(\mathcal{C})\left(\right.$ resp. $\left.\widetilde{F}_{I}(\tilde{\mathcal{C}})\right)$ par récurrence sur $|I|$ :

$$
\begin{aligned}
& F_{I}^{c}(\mathcal{C})=F\left(F_{I^{\prime}}^{c}(\mathcal{C})\right)\left(\operatorname{resp} . \widetilde{F}_{I}(\tilde{\mathcal{C}})=\widetilde{F}\left(\widetilde{F}_{I^{\prime}}(\tilde{\mathcal{C}})\right)\right) \text { où } I^{\prime}=I \backslash\{i\} \\
& \text { et } i=\max I .
\end{aligned}
$$

On vérifie que $\widetilde{F}_{I}(\tilde{\mathcal{C}})$ est équivalente à la catégorie des foncteurs contravariants de $(\boldsymbol{Z} \cup\{-\infty\})^{I}$ dans $\tilde{\mathcal{C}}$, donc indépendante de l'ordre choisi de $I$. Si $\mathcal{C}$ est une catégorie abélienne, cette définition de $F_{I}^{c}(\mathcal{C})$ coïncide avec celle de 1.1.13 d'après 1.2.12. En général, $F_{I}^{c}(\mathcal{C})$ est identifiée avec une sous-catégorie pleine de $F_{I}^{c}(\tilde{\mathcal{C}})$ définie par:

$$
\begin{aligned}
& \left(E,\left\{F_{i}\right\}\right) \in F_{I}^{c}(\mathcal{C}) \text { sii }\left(F_{i_{i}}^{p_{i}} / F_{i}^{q_{i}}\right) \cdots\left(F_{k}^{p_{k}} / F_{k^{k}}^{q_{k}}\right) E \in \mathcal{C} \\
& \text { pour tout }-\infty \leqslant p_{i}<q_{i} \leqslant+\infty(i \in I) ;
\end{aligned}
$$

(en particulier, $F_{I}^{c}(\mathcal{C})$ est indépendant de l'ordre de $I$ ), car on a le lemme suivant:

1.3.4. Lemme. Soient $\mathcal{C}$ et $\mathcal{C}^{\prime}$ des catégories exactes, supposons que $\mathcal{C}^{\prime}$ 
est une sous-catégorie pleine de $\mathcal{C}$, stable par extensions et qu'une suite courte de $\mathcal{C}^{\prime}$ est exacte sii elle l'est dans $\mathcal{C}$; alors $F\left(\mathcal{C}^{\prime}\right)$ est une sous-catégorie pleine de $F(C)$ telle que

$$
(E, F) \in F\left(C^{\prime}\right) \text { sii }\left(F^{p} / F^{q}\right) E \in \mathcal{C}^{\prime} \quad(-\infty \leqslant p<q \leqslant+\infty) ;
$$

donc $F\left(\mathcal{C}^{\prime}\right)$ est stable par extensions dans $F(\mathcal{C})$ et une suite courte de $F\left(\mathcal{C}^{\prime}\right)$ est strict sii elle l'est dans $F(C)$.

1.3.5. Soit $(E, d)$ un complexe d'une catégorie exacte $\mathcal{C}$, on dit que $E^{\circ}$ est strict, si $d^{i}: E^{i} \rightarrow E^{i+1}$ est strict $(i \in \mathbb{Z})$ et $\operatorname{Im} d^{i-1} \rightarrow \operatorname{Ker} d^{i}$ est un monomorphisme strict $(i \in \mathbb{Z})$.

Soit $\tilde{\mathcal{C}}$ comme dans 1.3 .2 , on dit que $(E, d)$ est faiblement strict rel. à $\tilde{C}$ si $H^{i} E \in \mathcal{C}(i \in \mathbb{Z})$, où $H^{i} E=\operatorname{Ker} d^{i} / \operatorname{Im} d^{i-1}$ dans $\tilde{\mathcal{C}}$.

Considérons la condition suivante:

(1.3.5.1) Si $v u$ est un monomorphisme strict, alors $u$ l'est.

Si (1.3.5.1) est satisfaite, alors $(E, d)$ est strict sii $d^{i}$ sont strict. La condition (1.3.5.1) est satisfaite pour $\mathcal{C}=F_{I}^{c}(\mathcal{A})$ où $\mathcal{A}$ est une catégorie abélienne, et un complexe de $F_{I}^{c}(\mathcal{A})$ est strict sii il est faiblement strict rel. à $\widetilde{F}_{I}(\mathscr{A})$, d'après 1.2.4.

1.3.6. Soit $\left(E^{\bullet}, W\right)$ un complexe de $F(C)$. Supposons que $W$ est finie sur chaque $E^{i}$. On a alors une suite spectrale dans $\tilde{\mathcal{C}}$ :

$$
E_{0}^{p q}=G r_{W}^{p} E^{p+q} \Rightarrow H^{p+q} E^{\bullet} .
$$

Si la condition:

$S(r):$ les complexes

$$
\left\{\cdots \rightarrow E_{r}^{p-r, q+r-1} \stackrel{d_{r}}{\rightarrow} E_{r}^{p, q} \stackrel{d_{r}}{\rightarrow} E_{r}^{p+r, q-r+1} \rightarrow \cdots\right\}
$$

sont faiblement stricts rel. à $\mathcal{C}\left(\right.$ donc $\left.E_{r+1}^{p q} \in \tilde{\mathcal{C}}\right)$,

est de proche en proche satisfaite pour tout $r \geqq 0$, alors

$$
G r_{W}^{p} H^{p+q} E \simeq E_{\infty}^{p q} \in \mathcal{C}, \quad \text { donc } H^{p+q} E \in \mathcal{C},
$$

i.e. le complexe $E^{\bullet}$ est faiblement strict rel. à $\tilde{\mathcal{C}}$, et $W$ est une filtration de $H^{p+q} E \in \mathcal{C}$ (comparer à ([6; (1.3.16), (7.2.5)]).

1.3.7. Soit $\left(E^{\circ}, W\right)$ un complexe de $F(\mathcal{C})$. Si la condition:

$$
d: G r_{W}^{p} E^{i} \rightarrow G r_{W}^{p} E^{i+1} \text { est strict pour tout } i, p,
$$

est satisfaite, $d: W^{p} E^{i} \rightarrow E^{i+1} / W^{p+1} E^{i+1}$ sont stricts car 


$$
\left.W^{p} E^{i} \rightarrow G r_{W}^{p} E^{i} \quad \text { (resp. } G r_{W}^{p} E^{i+1} \rightarrow E^{i+1} / W^{p+1} E^{i+1}\right)
$$

sont des épi- (resp. mono-) morphismes stricts; on a donc

$$
\operatorname{Dec}(W)^{p} E^{i}:=\operatorname{Ker}\left(d: W^{p+i} E^{i} \rightarrow E^{i+1} / W^{p+i+1} E^{i+1}\right) \in \mathcal{C}
$$

(cf. [6.(1.3.3)]). De plus, $\operatorname{Dec}(W)^{p} E^{i} \rightarrow \operatorname{Dec}(W)^{p-1} E^{i}\left(\operatorname{resp} . \operatorname{Dec}(W)^{p} E^{i} \rightarrow E^{i}\right)$ est un monomorphisme strict, $\operatorname{car} \operatorname{Dec}(W)^{p} E^{i} \rightarrow W^{p+i} E^{i}$ l'est et on a une suite exacte dans $C$ :

$$
0 \rightarrow W^{p+i} E^{i} \rightarrow \operatorname{Dec}(W)^{p-1} E^{i} \rightarrow \operatorname{Ker}\left(G r_{W}^{p+i-1} d^{i}\right) \rightarrow 0 ;
$$

donc on obtient une filtration $\operatorname{Dec}(W)$ de $E^{\circ}$. Notons que "Dec" commute à un foncteur exact, i.e. si $\phi: \mathcal{C} \rightarrow \mathcal{C}^{\prime}$ est un foncteur exact de catégories exactes, on a

$$
(\phi(E), \phi(\operatorname{Dec} W)) \simeq(\phi(E), \operatorname{Dec}(\phi W)) .
$$

$\mathrm{Si}$, de plus, la condition $S(r)$ de 1.3 .6 pour $(E, W)$ est satisfaite pour $r \leqslant 1$, alors les complexes $G r_{\text {Dec } W}^{p} E$ sont faiblement stricts rel. à $\tilde{C}$ d'après [6.(1.3.4)]; en particulier, si $\left(K_{A},\left(L_{A \otimes Q}, W\right),\left(K_{C} ; W, F\right)\right)$ est un complexe de Hodge mixte [6.(8.1.5.)], alors $\left(K_{C} ;\right.$ Dec $\left.W, F\right)$ est strict d'après 1.2.10, car $\left(K_{C}\right.$, Dec $W$ ) et $\left(G r_{\text {Dec } W}^{p} K_{C}, F\right)$ sont stricts (cf. 1.3.5).

1.3.8. Soit $\left(E^{\bullet} L ; W\right)=\left(\left(E^{*}, L\right), W\right)$ un complexe de $F_{I}^{c}(\mathcal{C})=F(F(\mathcal{C}))$ (i.e. $\left.|I|=2\right)$. Supposons que la filtration $L$ est finie sur chaque $E^{i}$, que les complexes filtrés $G r_{W}^{p}(E, L)$ appartiennent à $C^{s} F(\tilde{\mathcal{C}})$ pour tout $p$, et que la condition 1.3.7.1 est satisfaite pour les complexes $\left(G r_{L}^{j} E, W\right)$; alors la condition 1.3.7.1 est satisfaite pour $((E, L), W)$, donc on obtient un complexe de $F_{I}^{c}(C)$ par:

$$
(E ; L, \operatorname{Dec} W)=((E, L), \operatorname{Dec} W) ;
$$

de plus, on a

$$
\operatorname{Dec}(W)^{p}\left(L^{j} E\right)=\operatorname{Dec}(W)^{p} L^{j} E \quad \text { et } \quad \operatorname{Dec}(W)^{p}\left(G r_{L}^{j} E\right)=\operatorname{Dec}(W)^{p} G r_{L}^{j} E
$$

où $\left(L^{j} E, \operatorname{Dec} W\right)$ (resp. $\left(G r_{L}^{j} E, \operatorname{Dec} W\right)$ ) est defini pour $\left(L^{j} E, W\right)$ (resp. $\left.\left(G r_{L}^{j} E, W\right)\right)$.

En effet, $G r_{W} d^{i}: G r_{W}^{p}\left(E^{i}, L\right) \rightarrow G r_{W}^{p}\left(E^{i+1}, L\right)$ sont stricts dans $F(\tilde{\mathcal{C}})$ d'après 1.2.3, donc il suffit de vérifier que $\operatorname{Ker} G r_{L} G r_{W} d^{i}$, Coker $G r_{L} G r_{W} d^{i}$ et $\operatorname{Im} G r_{L}{ }^{\bullet}$ $G r_{W} d^{i}$ appartiennent à $\mathcal{C}$, mais cela résulte de l'hypothèse. La deuxième résulte $\mathrm{du}$ fait que le foncteur

$$
F(\mathcal{C}) \rightarrow \mathcal{C} \text { défini par }(E, L) \mapsto L^{j} E \text { (ou }(E, L) \mapsto G r_{L}^{j} E \text { ) }
$$

est un foncteur exact.

Considérons la suite spectrale dans $\tilde{F}(\tilde{\mathcal{C}})$ : 


$$
E_{0}^{p q}=G r_{L}^{p}\left(E^{p+q}, \operatorname{Dec} W\right) \Rightarrow H^{p+q}(E, \operatorname{Dec} W) .
$$

Si la condition $S(r)$ pour la suite spectrale (1.3.8.1) est satisfaite pour tout $r \geqslant 0$, on a alors $E_{\infty}^{p q}=G r_{L}^{p} H^{p+q}(E$, Dec $W) \in F(C)$, i.e. $L$ est une filtration de $H^{p+q}(E, \operatorname{Dec} W) \in F(\mathcal{C})$ dans $F(\mathcal{C})$. Supposons que $W$ est finie sur chaque $E^{i}$, alors, d'après [6.(1.3.2), (1.3.4)], la condition $S(0)$ est satisfaite pour (1.3.8.1), si la suite spectrale dans $\tilde{C}$ :

$$
E_{0}^{p q}=G r_{W}^{p} G r_{L}^{j} E^{p+q} \Rightarrow H^{p+q} G r_{L}^{j} E
$$

dégénère en $E_{2}$ pour tout $j$, et si la condition $S(r)$ pour la suite spectrale (1.3.8.2) est satisfaite pour $r \leqslant 1, j \in \mathbb{Z}$ (car un complexe $\left(E^{\prime}, F\right)$ de $F(C)$ est faiblement strict rel. à $\tilde{F}(\tilde{\mathcal{C}})$, sii $\left(E^{\prime}, F\right)$ est strict dans $F(\tilde{\mathcal{C}})$ et les $G r_{F}^{i} E^{\prime}$ sont faiblement stricts rel. à $\tilde{C}$, où $E^{\prime}=G r_{L}^{j} E$ et $\left.F=\operatorname{Dec} W\right)$, comparer à [6.(8.1.15)], où $S(r)$ pour (1.3.8.1) est satisfaite pour $r \geqslant 1$ d'après l'existence de structures de Hodge mixtes.

1.3.9. Soient $M$ un objet d'une catégorie abélienne, muni d'une filtration finie croissante $L$, et $N$ un endomorphisme nilpotent de $M$ qui respecte $L$. D'apres [7.(1.6.13)] il existe au plus une filtration finie croissante $W$ de $M$ telle que $N W_{i} M \subset W_{i-2} M$ et que

$$
N^{k}: G r_{i+k}^{W} G r_{i}^{L} M \underset{\rightarrow}{\longrightarrow} G r_{i-k}^{W} G r_{i}^{L} M,
$$

de plus, on a une formule inductive (si elle existe):

$$
\begin{array}{ll}
W_{-i+k} L_{k} M=W_{-i+k} L_{k-1} M+N^{i} W_{i+k} L_{k} M & i>0, \\
W_{i+k} L_{k} M=\operatorname{Ker}\left(N^{i+1}: L_{k} M \rightarrow L_{k} M / W_{-i-2+k} L_{k} M\right) & i \geqslant 0 .
\end{array}
$$

On dit que $W$ est la filtration associée à $N$ relativement à $L$. Si $G r_{i}^{W} M=0$ pour $i \neq n$, on dit que $W$ est la filtration associée à $N$ décalée par $n$.

1.3.10. Soit $E$ un objet d'une catégorie exacte $\mathcal{C}$, muni d'une filtration finie croissante $L$, soit $N$ un endomorphisme nilpotent de $(E, L) \in F(C)$. D'après 1.3.9, il existe au plus une filtration finie croissante $W$ de $(E, L) \in F(C)$ telle que $N$ induit un morphisme $N:\left(E^{\circ} ; L, W\right) \rightarrow\left(E^{\bullet} L ; W[2]\right)$ et que (1.3.9.1) soit satisfaite en remplaçant $M$ par $E$, où $(W[2])_{i}=W_{i-2}$ car $W$ est croissante.

Soit $u:\left(\left(E_{1}, L\right), N\right) \rightarrow\left(\left(E_{2}, L\right), N\right)$ un morphisme de tels deux objets, i.e. $u$ est un morphisme de $F(C)$, compatible avec l'action de $N$; alors $u$ induit un (unique) morphisme $\tilde{u}:\left(E_{1}, L ; W\right) \rightarrow\left(E_{2} ; L, W\right)$ de $F(F(C))$, car c'est valable dans $F(F(\tilde{\mathcal{C}}))$ d'après (1.3.9.2). Si, de plus, $\tilde{u}$ est strict, la filtration $W$ sur $\operatorname{Ker} u, \operatorname{Im} u$, Coker $u$ est la filtration associée à $N$ relativement à $L$.

1.3.11. Soit $\left(\left(E^{\circ}, L\right), W\right)$ un complexe de $F(F(C))$. Supposons que toutes les 
hypothèse de 1.3.8 soient satisfaites, que la suite spectrale (1.3.8.1) dégénère en $E^{\prime}$ et qu'il existe un morphisme $N:\left(E^{\cdot} ; L, W\right) \rightarrow\left(E^{*}, L ; W[2]\right)$ dans la catégorie dérivée, tel que l'action de $N$ sur chaque $H^{i} G r_{j}^{L} E$ soit nilpotente et que la filtration décalée Dec $W$ sur $H^{i} G r_{j}^{L} E$ soit la filtration associée à $N$ décalée par $i+j$, où $L_{j}=L^{-j}, W_{i}=W^{-i}$ et $(\operatorname{Dec} W)_{i}=(\operatorname{Dec} W)^{-i}$. (Notons que le complexe $G_{j}^{L}(E, \operatorname{Dec} W)$ est faiblement strict rel. à $\tilde{\mathcal{C}}$ par la condition $S(0)$ pour (1.3.8.1).) Alors, d'après 1.3.8, $E_{\infty}^{p q}=G r_{-p}^{L} H^{p+q}(E$, Dec $W) \in$ $F(C)$, i.e. on obtient $\left(H^{i} E ; L, \operatorname{Dec} W\right) \in F(F(\mathcal{C}))$, de plus la filtration Dec $W$ sur $H^{i} E$ est la filtration associée à $N$ relativement à $\operatorname{Dec} L$, car le morphisme

$$
d_{1}: H^{i} G r_{j}^{L}(E, \operatorname{Dec} W) \rightarrow H^{i+1} G r_{j-1}^{L}(E, \operatorname{Dec} W)
$$

est compatible à l'action de $N$, et est strict par hypothèse (donc la filtration Dec $W$ sur $E_{\infty}^{p q}=G r_{-p}^{L} H^{p+q} E$ est la filtration associée à $N$ décalée par q.)

1.3.12. Remarque. Soit $\mathcal{C}$ une catégorie exacte, on dit que $\mathcal{C}$ est semi-simple, si toutes les suites exactes de $\mathcal{C}$ scindent. Si $\mathcal{C}$ eșt semi-simple, on vérifie que $F_{f}(C)$ la catégorie des objet de $\mathcal{C}$ munis d'une filtration finie est semi-simple, et que $\phi:\left(E_{1}, F\right) \rightarrow\left(E_{2}, F\right)$ un morphisme de $F_{f}(C)$ est strict sii $\phi$ est isomorphe à la somme directe de morphismes stricts dans $\mathcal{C}: \phi_{i}: E_{1}^{i} \rightarrow E_{2}^{i}$ tels que $F^{p}\left(E_{j}\right) \simeq$ $\bigoplus_{i \geqslant p} E_{j}^{i}(j=1,2)$. En effet, pour une suite exacte de $F_{f}(\mathcal{C})$ :

$$
0 \rightarrow\left(E_{-1}, F\right) \rightarrow\left(E_{0}, F\right) \rightarrow\left(E_{1}, F\right) \rightarrow 0
$$

et pour $\bigoplus_{i} E_{ \pm 1}^{i}$ un scindage de $\left(E_{ \pm 1}, F\right)$, on a $\bigoplus_{i} E_{0}^{i}$ un scindage de $\left(E_{0}, F\right)$ compatible avec $(1.3 .12 .1)$ et avec les scindages de $\left(E_{ \pm 1}, F\right)$, tel que les suites exactes de $C$ :

$$
0 \rightarrow E_{-1}^{i} \rightarrow E_{0}^{i} \rightarrow E_{1}^{i} \rightarrow 0
$$

se scindent.

En particulier, si $\mathcal{A}$ est une catégorie abélienne semi-simple, e.g. celle des espaces vectoriels sur un corps, $F_{I f}^{c}(\mathcal{A})$ la catégorie des objets de $\mathcal{C}$, munis de $I$-filtrations finies compatibles est semi-simple; un objet $\left(A, F_{i}(i \in I)\right)$ de $F_{I f}^{c}(\mathcal{A})$ est isomorphe à $\left(\underset{\nu \in Z^{I}}{\bigoplus^{\nu}} B_{i}^{\nu} F_{i}(i \in I)\right)$ où $F_{i}^{p}\left(\oplus B^{\nu}\right)=\bigoplus_{\nu_{i} \geqslant p} B^{\nu}$, et $\left(A^{\bullet}, F_{i}\right.$ $(i \in I)$ ) un complexe de $F_{I f}^{c}(\mathcal{A})$ est strict sii $A^{*}$ est isomorphe à $\bigoplus_{\nu} B^{\nu^{*}}$ (dans la catégorie de complexes) tel que $F_{i}^{p} A^{*} \cong{ }_{\nu_{i} \geqslant p} B^{\nu^{*}}$.

\section{§2. DD-Modules Filtrés}

(2.0.1) On suppose que toutes les variétés complexes lisses considérées dans 
cet article sont paracompactes et de dimension pure. La dimension de $X$ sera notée $d_{X}$.

(2.0.2) Soit $n \in \mathbb{Z}$, on définit le "twist de Tate" par:

$$
M(n)=M \otimes_{Z} \mathbb{Z}(n) \text { pour } M \text { un } \mathbb{Z} \text {-Module, }
$$

où $\boldsymbol{Z}(n):=(2 \pi i)^{n} \boldsymbol{Z} \subset \boldsymbol{C}$. Si $M$ est un $\boldsymbol{C}$-Module, on a un isomorphisme canonique:

$$
M(n) \stackrel{\sim}{\rightarrow} M
$$

induit par l'isomorphisme: $\boldsymbol{C}(n) \stackrel{\sim}{\rightarrow}$.

\section{1. $\mathscr{D}$-Modules filtrés et $\mathscr{B}$-Modules gradués.}

2.1.1. Soit $X$ une variété complexe lisse. Soit $\mathscr{D}_{X}$ l'Anneau des opérateurs différentiels linéaires sur $X$, muni de la filtration $F$ par l'ordre des opérateurs. On définit un Anneau gradué sur $X$ par $\mathscr{B}=\bigoplus_{p} F_{p} \mathscr{D}_{X}$ (cf. [12]).

Soit $\operatorname{MF}\left(\mathscr{D}_{X}\right)$ la catégorie additive des $\mathscr{D}_{X}$-Modules (à droite) filtrés, dont les objets $(M, F)$ satisfaient les conditions:

(2.1.1.1) $\bigcup_{p} F_{p} M=M$,

(2.1.1.2) $\quad F_{p} M=0$ pour $p \ll 0$ localement sur $X$,

(2.1.1.3) $\left(F_{p} M\right)\left(F_{q} \mathscr{D}_{X}\right) \subset F_{p+q} M$ pour tout $\mathrm{p}, q \in \mathbb{Z}$,

et dont les morphismes sont $\mathscr{D}_{X}$-linéaires et respectent la filtration.

Soit $M G(\mathscr{B})$ la catégorie abélienne des $\mathscr{B}$-Modules (à droite) gradués dont les objets $M$. satisfaient la condition:

$$
M_{p}=0 \text { pour } p \ll 0 \text { localement sur } X .
$$

On a alors un foncteur canonique:

$$
\oplus: M F\left(\mathscr{D}_{X}\right) \rightarrow M G(\mathscr{B}) \quad \text { (resp. } \lim : M G(\mathscr{B}) \rightarrow M F(\mathscr{D}) \text { ) }
$$

défini par:

$$
\bigoplus(M, F)=\bigoplus_{p} F_{p} M \quad\left(\text { resp. } \lim M_{\bullet}=\left(\lim _{\longrightarrow} M_{i}, F\right)\right)
$$

où $F_{p}\left(\lim _{\rightarrow} M_{i}\right)=\operatorname{Im}\left(F_{p} M \rightarrow \underset{\rightarrow}{\rightarrow} M_{i}\right)$. Notons que les morphismes $M_{i} \rightarrow M_{i+1}$ sont induits par l'action de $1 \in \mathscr{B}_{1}=F_{1} \mathscr{D}_{X}$.

2.1.2. Remarque. La catégorie $M F\left(\mathscr{D}_{X}\right)$ s'identifie, par le foncteur $\oplus$, à la sous-catégorie pleine de $M G(\mathscr{B})$ des objets sur lesquels l'action de $1 \in \mathscr{B}_{1}$ est injective.

2.1.3. Soit $(L, F)$ un $\mathcal{O}_{X}$-Module filtré tel que $F_{p} L=0$ pour $p \ll 0$ localement 
sur $X$. On définit un $\mathscr{D}_{X}$-Module filtré $(M, F)$ (resp. un $\mathscr{B}$-Module gradué $M$.), noté $(L, F) \otimes_{\mathcal{O}}\left(\mathscr{D}_{X}, F\right)$ (resp. $\left.(L, F) \otimes_{\mathcal{O}} \mathscr{B}\right)$, par:

$$
M=L \otimes_{\mathcal{O}} \mathscr{D}_{X}, \quad F_{p} M=\sum_{i} F_{p-i} L \otimes F_{i} \mathscr{D}_{X} \quad\left(\text { resp. } M_{p}=\sum_{i} F_{p-i} L \otimes F_{i} \mathscr{D}_{X}\right) .
$$

On dit que $(M, F) \in M F\left(\mathscr{D}_{X}\right)$ (resp. $M_{\bullet} \in M G(\mathscr{B})$ ) est un Module induit s'il est isomorphe à $(L, F) \otimes\left(\mathscr{D}_{X}, F\right)$ (resp. $\left.(L, F) \otimes \mathscr{B}\right)$ pour un $\mathcal{O}_{X}$-Module filtré $(L, F)$ comme ci-dessus. On notera $M F_{i}\left(\mathscr{D}_{X}\right)$ (resp. $M G_{i}(\mathscr{B})$ ) la souscatégorie pleine de $M F\left(\mathscr{D}_{X}\right)$ (resp. $M G(\mathscr{B})$ ) des Modules induits.

On dit que $(M, F)$ (resp. $M$.) est un Module induit de degré $p$, s'il est isomorphe à $(L, F) \otimes\left(\mathscr{D}_{X}, F\right)$ (resp. $\left.(L, F) \otimes \mathscr{B}\right)$ avec $G r_{i}^{F} L=0$ pour $i \neq p$; dans ce cas, celui-ci est noté aussi $L \otimes\left(\mathscr{D}_{X}, F[p]\right)$ (resp. $L \otimes \mathscr{B}(p)$ ).

2.1.4. Remarque. Le foncteur $\oplus$ établit une équivalence de catégories: $M F_{i}\left(\mathscr{D}_{X}\right) \stackrel{\sim}{\rightarrow} M G_{i}(\mathscr{B})$.

2.1.5. Soit $\left(\mathscr{D}_{X} \otimes_{\mathcal{O}} \Lambda^{-\cdot} \Theta_{X}, F\right) \rightarrow\left(\mathcal{O}_{X}, F\right)$ la résolution canonique par des $\mathscr{D}_{X^{-}}$ Modules (à gauche) filtrés (cf. [18]), i.e. le premier complexe est localement isomorphe au complexe de $\operatorname{Koszul} K\left(\mathscr{D}_{X} ; \cdot \partial_{i}\left(1 \leqq i \leqq d_{X}\right)\right)\left[d_{X}\right]$ (où $\cdot \partial_{i}$ signifie la multiplication à droite), dont la filtration s'écrit:

$$
F_{p}\left(\mathscr{D}_{X} \otimes \Lambda^{-i} \Theta_{X}\right)=F_{p+i} \mathscr{D}_{X} \otimes \Lambda^{-i} \Theta_{X},
$$

et la filtration $F$ de $\mathcal{O}_{X}$ est définie par $G r_{p}^{F} \mathcal{O}_{X}=0$ pour $p \neq 0$. Ici, le morphisme entre les complexes est défini par la surjection canonique: $\mathscr{D}_{X} \ni P \mapsto P 1 \in \mathcal{O}_{X}$. Soit $(M, F) \in M F\left(\mathscr{D}_{X}\right)$, on a alors un complexe de $M F\left(\mathscr{D}_{X}\right)$ :

$$
(M, F) \otimes_{\mathcal{O}}\left(\mathscr{D}_{X} \otimes \Lambda^{-\cdot} \Theta_{X}, F\right)
$$

et un morphisme canonique de ce complexe filtré dans $(M, F)$.

2.1.6. Lemme. Les $\mathscr{D}_{X}$-Modules filtrés $(M, F) \otimes_{\mathcal{O}}\left(\mathscr{D}_{X} \otimes \Lambda^{-i} \Theta_{X}, F\right)$ sont des Modules induits, et le morphisme canonique:

$$
(M, F) \otimes_{\mathcal{O}}\left(\mathscr{D}_{X} \otimes \Lambda^{-\cdot \Theta_{X}}, F\right) \rightarrow(M, F)
$$

est un quasi-isomorphisme filtré.

Preuve. Posons $L=\Lambda^{-i} \Theta_{X}$, alors le morphisme canonique

$$
\left(M \otimes_{\mathcal{O}} L, F[i]\right) \hookrightarrow(M, F) \otimes_{\mathcal{O}}\left(\mathscr{D}_{X} \otimes L, F[i]\right)
$$

induit un morphisme $\mathscr{D}_{X}$-linéaire filtré:

$$
\left(M \otimes_{\mathcal{O}} L, F[i]\right) \otimes_{\mathcal{O}}\left(\mathscr{D}_{\mathrm{X}}, F\right) \rightarrow(M, F) \otimes_{\mathcal{O}}\left(\mathscr{D}_{\mathrm{X}} \otimes L, F[i]\right) .
$$

On vérifie facilement que c'est un isomorphisme filtré (on peut aussi utiliser le 
lemme 2.4.2); d'où la première assertion. Par définition, le gradué associé du premier complexe de (2.1.6.1) est localement isomorphe au complexe de Koszul:

$$
K\left(G r^{F} M \otimes G r^{F} \mathscr{D}_{X} ; g r \partial_{i} \otimes 1-1 \otimes g r \partial_{i} \quad\left(1 \leqq i \leqq d_{X}\right)\right)\left[d_{X}\right],
$$

donc, en utilisant la filtration par le degré de $g r \partial_{i} \in G r^{F} \mathscr{D}_{X}$, on voit que le gradué associé de (2.1.6.1) est un quasi-isomorphisme.

2.1.7. Corollaire. Soit $(M, F) \in M F\left(\mathscr{D}_{X}\right)$ (resp. $M_{\bullet} \in M G(\mathscr{B})$ ), alors il existe une résolution finie, canonique et fonctorielle:

$$
\left(L^{\bullet}, F\right) \stackrel{\sim}{\rightarrow}(M, F) \quad\left(\text { resp. } L_{\bullet}: \underset{\rightarrow}{\rightarrow} M_{\bullet}\right)
$$

par des Modules induits.

Preuve. Soit $M_{\bullet} \in M G(\mathscr{B})$. Posons $L_{\bullet}=\oplus_{p} M_{p} \otimes_{\mathcal{O}} \mathcal{B}(p)$. Alors $L_{\text {c }}$ est un Module induit, et on a une suite exacte dans $M G(\mathscr{B})$ :

$$
0 \rightarrow K_{\bullet} \rightarrow L_{\bullet} \rightarrow M_{\bullet} \rightarrow 0 \text { 。 }
$$

Puisque l'action de $1 \in \mathscr{B}_{1}$ est injective sur $K_{\bullet}$, l'assertion est réduite au cas de $M F\left(\mathscr{D}_{X}\right)$, donc cela résulte du lemme 2.1.6.

2.1.8. Soit $\longleftarrow=b,+,-$ ou $\infty$. Soient

$$
C^{\natural} F\left(\mathscr{D}_{X}\right)=C^{\varkappa}\left(M F\left(\mathscr{D}_{X}\right)\right) \text { et } \quad K^{\varkappa} F\left(\mathscr{D}_{X}\right)=K^{\Downarrow}\left(M F\left(\mathscr{D}_{X}\right)\right)
$$

comme dans [31], où $C^{\infty}$ (resp. $K^{\infty}$ ) sera noté aussi $C$ (resp. $K$ ). Ici, on suppose que la condition (2.1.1.2) pour chaque composante $\left(M^{i}, F\right)$ des objets de $C^{\grave{\imath}} F\left(\mathscr{D}_{X}\right)$ (et de $K^{\grave{\imath}} F\left(\mathscr{D}_{X}\right)$ ) est satisfaite uniformément pour $i$. Pour $*=b$, + , - ou $\emptyset$, on notera $K^{\downarrow} F\left(\mathscr{D}_{X}\right)^{*}$ la sous-catégorie pleine de $K^{\downarrow} F\left(\mathscr{D}_{X}\right)$ des objets $\left(M^{\circ}, F\right)$ satisfaisant la condition:

$$
\mathscr{H}^{i} G r^{F} M^{\bullet}=0 \text { pour } \begin{cases}|i| \gg 0 & \text { si } *=b \\ i \ll 0 & \text { si } *=+ \\ i \gg 0 & \text { si } *=- \\ \text { tout } i & \text { si } *=\emptyset\end{cases}
$$

Posons $K^{\text {々 }} F\left(\mathscr{D}_{X}\right)^{\infty}=K^{\varkappa} F\left(\mathscr{D}_{X}\right)$. Pour $\xi, *=b,+,-$ ou $\infty$, on définit

$$
D^{\natural} F\left(\mathscr{D}_{X}\right)^{*}=K^{\varkappa} F\left(\mathscr{D}_{X}\right)^{*} / K^{\natural} F\left(\mathscr{D}_{X}\right)^{\emptyset} \text {. }
$$

Posons $D^{\natural} F\left(\mathscr{D}_{X}\right)=D^{\natural} F\left(\mathscr{D}_{X}\right)^{\infty}$ et $D F\left(\mathscr{D}_{X}\right)^{*}=D^{\infty} F\left(\mathscr{D}_{X}\right)^{*}$. De même, on définit $D^{\star} G(\mathscr{B})^{*}$, etc., en remplaçant $\mathscr{H}^{i} G r^{F}$ par $\mathscr{H}^{i}$ dans la condition (2.1.8.9).

2.1.9. Lemme. Pour $\succsim, *=b,+,-$ ou $\infty, K^{\natural} F\left(\mathscr{D}_{X}\right)^{\emptyset}$ est une sous-caté- 
gorie épaisse de $K^{\Uparrow} F\left(\mathscr{D}_{X}\right)^{*}$ (donc $D^{\natural} F\left(\mathscr{D}_{X}\right)^{*}$ est une catégorie triangulée, cf. [31]). De plus $D^{\Downarrow} F\left(\mathscr{D}_{X}\right)^{*}$ est une sous-catégorie triangulée pleine de $D F\left(\mathscr{D}_{X}\right)$, et le foncteur naturel: $D^{\natural} F\left(\mathscr{D}_{X}\right) \rightarrow D F\left(\mathscr{D}_{X}\right)^{\text {in }}$ est une équivalence de catégories. (De même pour $D G(\mathscr{B})$ ).

Preuve. Par le même argument que dans [loc. cit.], il suffit de définir le triangle de $K^{\text {i }} F\left(\mathscr{D}_{X}\right)$ :

$$
\rightarrow \tau_{\leqq i}\left(M^{*}, F\right) \rightarrow\left(M^{\cdot}, F\right) \rightarrow \tau_{>i}\left(M^{*}, F\right) \rightarrow
$$

tel que $\tau_{\leqq i}\left(M^{*}, F\right)$ (resp. $\left.\tau_{>i}\left(M^{*}, F\right)\right) \in K^{\natural} F\left(\mathscr{D}_{X}\right)^{\emptyset}$ pour $i \ll 0$ (resp. $i \gg 0$ ), si $\left(M^{*}, F\right) \in K^{\natural} F\left(\mathscr{D}_{X}\right)^{+}$(resp. $\left.K^{\natural} F\left(\mathscr{D}_{X}\right)^{-}\right)$. Donc il suffit de vérifier que $d^{i}$ : $\left(M^{i}, F\right) \rightarrow\left(M^{i+1}, F\right)$ est strict pour $i \ll 0$ (resp. $\left.i \gg 0\right)$, si $\left(M^{\bullet}, F\right)$ est comme cidessus; mais c'est évident (cf. la preuve de 1.2.4; voir aussi [21].) L'assertion pour $D G(\mathscr{B})$ est évidente.

2.1.10. Soit $(M, F) \in M F_{i}\left(\mathscr{D}_{X}\right)$, cf. 2.1.3. On définit une filtration $G \operatorname{de}(M, F)$ par:

$$
G_{i}(M, F)=\left(\left(F_{i} M\right) \mathscr{D}_{X}, F\right)
$$

où $F$ est la filtration induite $\operatorname{sur}\left(F_{i} M\right) \mathscr{D}_{X}$. Alors chaque $G_{i}^{G}(M, F)$ est un Module induit de degré $i$. Soit $M F_{i}^{f}\left(\mathscr{D}_{X}\right)$ la sous-catégorie pleine de $M F_{i}\left(\mathscr{D}_{X}\right)$ des objets satisfaisant la condition:

$$
G_{i}(M, F)=(M, F) \text { pour } i \gg 0 \text { localement sur } X .
$$

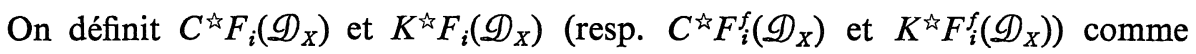
dans 2.1.8 (où la condition (2.1.10.1) est satisfaite uniformément). Alors

$$
K^{i} F_{i}^{(f)}\left(\mathscr{D}_{X}\right)^{\emptyset}=K^{i} F_{i}^{(f)}\left(\mathscr{D}_{X}\right) \cap K F\left(\mathscr{D}_{X}\right)^{\emptyset}
$$

est une sous-catégorie épaisse. Posons

$$
D^{\natural} F_{i}^{(f)}\left(\mathscr{D}_{X}\right)=K^{\natural} F_{i}^{(f)}\left(\mathscr{D}_{X}\right) / K^{\natural} F_{i}^{(f)}\left(\mathscr{D}_{X}\right)^{\emptyset}
$$

On définit similairement $D^{\natural} G_{i}^{(f)}(\mathscr{B})$ (où la filtration $G$ est définie par l'équivalence dans 2.1.4.), alors cette catégorie triangulée est équivalente à $D^{\text {仙 }} F_{i}^{(f)}\left(\mathscr{D}_{X}\right)$ par définition. Ici $(f)$ signifie qu'il y a deux énoncés l'un pour $K^{\text {in }} F_{i}^{f}\left(\mathscr{D}_{X}\right)$ etc. et l'autre pour $K^{\natural} F_{i}\left(\mathscr{D}_{X}\right)$ etc.

2.1.11. Lemme. $S i\left(M^{\bullet}, F\right) \in K^{\text {々 }} F_{i}\left(\mathscr{D}_{X}\right)^{\emptyset}$, on a $G r_{i}^{G}\left(M^{\bullet}, F\right) \in K^{\natural} F_{i}\left(\mathscr{D}_{X}\right)^{\emptyset}$ pour tout i. Donc, si $\left(M^{*}, F\right) \rightarrow\left(N^{*}, F\right)$ est un quasi-isomorphisme filtré dans $K^{\natural} F_{i}\left(\mathscr{D}_{X}\right)$, alors $G r_{i}^{G}\left(M^{\bullet}, F\right) \rightarrow G r_{i}^{G}\left(N^{*}, F\right)$ l'est pour tout $i$. 
Preuve. On a $G r_{i}^{G}\left(M^{\bullet}, F\right) \in K^{\Uparrow} F_{i}\left(\mathscr{D}_{X}\right)^{\emptyset}$ sii $G r_{i}^{F} G r_{i}^{G} M^{\bullet}$ est acyclique. Puisque $G r_{i}^{F} G r_{i}^{G} M^{\circ}=F_{i} M^{\bullet}$ si $i=\min \left\{i: F_{i} M^{\circ} \neq 0\right\}$ (ce qui existe localement), l'assertion résulte par récurrence sur $i$, en remplaçant $\left(M^{\circ}, F\right) \operatorname{par}\left(M^{\circ} / G_{i} M^{\circ}, F\right)$.

2.1.12. Proposition. Soit $\xi^{2}=b,+,-$ ou $\infty$ comme dans 2.1.8.

(1) Le foncteur $\oplus$ (cf. 2.1.1) induit les équivalences de catégories:

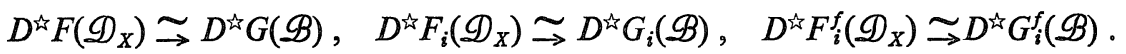

(2) Les foncteurs naturels:

$$
D^{\natural} F_{i}\left(\mathscr{D}_{X}\right) \rightarrow D^{\natural} F\left(\mathscr{D}_{X}\right), \quad D^{\natural} G_{i}(\mathscr{B}) \rightarrow D^{\natural} G(\mathscr{B})
$$

sont des équivalences de catégories.

(3) Les foncteurs naturels:

$$
D^{\natural} F_{i}^{f}\left(\mathscr{D}_{X}\right) \rightarrow D^{\varkappa} F_{i}\left(\mathscr{D}_{X}\right), \quad D^{\varkappa} G_{i}^{f}(\mathscr{B}) \rightarrow D^{\natural} G_{i}(\mathscr{B})
$$

sont pleinement fidèles.

Preuve. Les assertions (1) et (2) résultent de 2.1.4, 2.1.7 et de [31]. Prouvons l'assertion (3). Soit $\left(M_{2}^{\circ}, F\right) \rightarrow\left(M_{1}^{\circ}, F\right)$ un quasi-isomorphisme filtré avec

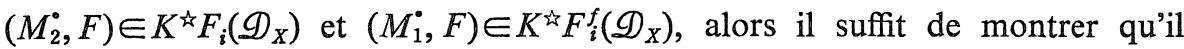
existe un quasi-isomorphisme filtré: $\left(M_{3}^{\circ}, F\right) \rightarrow\left(M_{2}^{\circ}, F\right)$ avec $\left(M_{3}^{\circ}, F\right) \in K^{\text {iे }} F_{i}^{f}\left(\mathscr{D}_{X}\right)$. Soit $X=\bigcup_{i} U_{i}$ un recouvrement localement fini de $X$, tel que

$$
\min \left\{p: G_{p} M_{1}^{\circ}=M_{1}^{\circ}\right\}
$$

existe sur chaque $U_{i}$. Soient $U_{I}=\bigcap_{i \in I} U_{i}, j_{I}: U_{I} \rightarrow X$ l'inclusion naturelle, et

$$
p(I)=\min \left\{p:\left.G_{p} M_{I}^{*}\right|_{U_{I}}=\left.M_{i}^{*}\right|_{U_{I}}\right\} .
$$

On définit $\left(M_{3}^{\circ}, F\right)$ par le complexe simple associé au complexe double de Čech dont la composante de degré $(k, l)$ est

$$
\bigoplus_{1-|I|=k}\left(j_{I}\right)_{!} j_{I}^{-1}\left(G_{p(I)} M_{2}^{l}, F\right)
$$

où la différentielle de Čech est induite par le morphisme naturel:

$$
\left(j_{I}\right)_{!} j_{I}^{-1}\left(G_{p(I)} M_{2}, F\right) \rightarrow\left(j_{I^{\prime}}\right)_{!} j_{I^{\prime}}^{-1}\left(G_{p\left(I^{\prime}\right)} M_{2}, F\right)
$$

pour $I \supset I^{\prime}$. On a alors un morphisme naturel: $\left(M_{3}^{\circ}, F\right) \rightarrow\left(M_{2}^{\circ}, F\right)$, ce qui est un quasi-isomorphisme filtré d'après 2.1.11; d'où l'assertion.

2.1.13. Remarque. La preuve de l'assertion (3) montre que la catégorie $D^{\varkappa} F_{i}^{f}\left(\mathscr{D}_{X}\right)$ est équivalente à la sous-catégorie pleine de $D^{\natural} F_{i}\left(\mathscr{D}_{X}\right)$ des objets 
$\left(M^{*}, F\right)$ satisfaisant la condition:

(2.1.13.1) $G r^{F} G r_{i}^{G}\left(M^{\bullet}, F\right)$ est acyclique pour $i \gg 0$ localement sur $X$.

2.1.14. Lemme. Soit

$$
D F_{i}^{(f)}\left(\mathscr{D}_{X}\right)^{\not{H}}=D F_{i}^{(f)}\left(\mathscr{D}_{X}\right) \cap D F\left(\mathscr{D}_{X}\right)^{\text {is }} \text { dans } \quad D F\left(\mathscr{D}_{X}\right),
$$

cf. 2.1.9, alors le foncteur naturel:

$$
D^{\natural} F_{i}^{(f)}\left(\mathscr{D}_{X}\right) \rightarrow D F_{i}^{(f)}\left(\mathscr{D}_{X}\right)^{\natural)}
$$

est une équivalence de catégories.

Preuve. Il suffit de vérifier que ce foncteur est essentiellement surjectif. D'après le remarque ci-dessus, on peut remplacer la condition (2.1.10.1) par la condition cohomologique (2.1.13.1) dans la définition de $D^{\text {« }} F_{i}^{f}\left(\mathscr{D}_{X}\right)$; donc il suffit de montrer la surjectivité essentielle de $D^{\natural} F_{i}\left(\mathscr{D}_{X}\right) \rightarrow D F_{i}\left(\mathscr{D}_{X}\right)^{\text {tr }}$; mais la première catégorie est équivalente à $D^{\natural} F\left(\mathscr{D}_{X}\right)$ et la deuxième est contenue dans $D F\left(\mathscr{D}_{X}\right)^{\text {i⿱ }}$, donc l'assertion résulte de 2.1.9.

2.1.15. Soit $D_{\mathrm{coh}}^{\grave{\imath}} F_{i}^{f}\left(\mathscr{D}_{X}\right)$ la sous-catégorie triangulée pleine de $D^{\natural} F_{i}^{f}\left(\mathscr{D}_{X}\right)$ des objets $\left(M^{*}, F\right)$ satisfaisant la condition:

(2.1.15.1) $\mathcal{H}^{j} G r_{i}^{F} G r_{i}^{G} M^{\bullet}$ sont des $\mathcal{O}_{X}$-Modules cohérents pour tout $i, j$. Notons que la différentielle de $G r_{i}^{F} G r_{i}^{G} M^{\bullet}$ est $\mathcal{O}_{X}$-linéaire et que $G r_{i}^{G}\left(M^{\bullet}, F\right) \cong$ $G r_{i}^{F} G r_{i}^{G} M^{\bullet} \otimes_{\mathcal{O}_{X}}\left(\mathscr{D}_{X}, F[i]\right)$ comme complexes de $\mathscr{D}_{X}$-Modules filtrés induits.

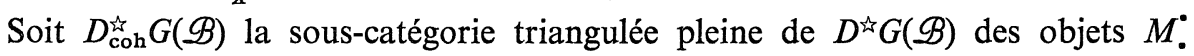
satisfaisant la condition:

(2.1.15.2) $\mathscr{H}^{j} M^{*}$. sont des $\mathscr{B}$-Modules gradués cohérents pour tout $j$. Posons

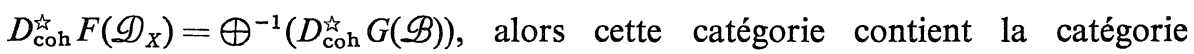
$D_{\text {coh }}^{\text {i⿱ }} F_{i}^{f}\left(\mathscr{D}_{X}\right)$ (par récurrence en utilisant la filtration $G$ ).

2.1.16. Proposition. Soit $\hat{\imath}=b$, alors les foncteurs naturels:

$$
D_{\mathrm{coh}}^{b} F_{i}^{f}\left(\mathscr{D}_{X}\right) \rightarrow D_{\mathrm{coh}}^{b} F\left(\mathscr{D}_{X}\right) \stackrel{\oplus}{\rightarrow} D_{\mathrm{coh}}^{b} G(\mathscr{B})
$$

sont des équivalences de catégories.

Preuve. Puisque toutes les catégories sont des sous-catégories triangulées pleines de $D G(\mathscr{B})$, il suffit de montrer que tout $\mathscr{B}$-Module gradué cohérent $M$. appartient essentiellement à $\oplus\left(D_{\text {coh }}^{b} F_{i}^{f}\left(\mathscr{D}_{X}\right)\right.$. Par la preuve de la proposition 2.1.12, on peut remplacer la condition (2.1.10.1) imposée sur les objets de 
$D_{\text {coh }}^{b} F_{i}^{f}\left(\mathscr{D}_{X}\right)$ par la condition cohomologique (2.1.13.1); donc l'asserion est locale d'après 2.1.11, car on a construit une résolution canonique en 2.1.7. Localement on a une suite exacte: $0 \rightarrow K_{\bullet} \rightarrow L_{\bullet} \rightarrow M_{\bullet} \rightarrow 0$, où $L_{\bullet}=\bigoplus_{p \leq p_{0}} M_{p} \otimes \mathscr{B}(p)$; donc l'assertion est réduite au cas $M_{\bullet}=\oplus(M, F)$ avec $G r^{F} M$ cohérent sur $G r^{F} \mathscr{D}_{X}$, et cela résulte du lemme suivant.

2.1.17. Lemme. Soit $(M, F) \in M F\left(\mathscr{D}_{X}\right)$ tel que $G r^{F} M$ est un $G r^{F} \mathscr{D}_{X^{-}}$ Module cohérent. On a alors localement une résolution: $\left(L^{\circ}, F\right) \rightarrow(M, F)$ de longueur $\leqq 2 d_{X}$ par des Modules induits libres de type fini, i.e. $\left(L^{j}, F\right)=$ $\oplus_{p} L_{p}^{j} \otimes_{\mathcal{O}_{X}}\left(\mathscr{D}_{X}, F[p]\right)$ avec $L_{p}^{j}$ un $\mathcal{O}_{X}$-Module libre de type fini, où $L_{p}^{j}=0$ sauf un nombre fini de $p$.

Preuve. Localement on a une résolution: $\left(L^{\cdot}, F\right) \rightarrow(M, F)$ avec $\left(L^{j}, F\right)$ induit libre de type fini d'après le théorème $\mathrm{A}$ de Cartan. Poson

$$
(K, F)=\operatorname{Ker}\left(\left(L^{-2 d} x^{+1}, F\right) \rightarrow\left(L^{-2 d} x^{+2}, F\right)\right),
$$

alors il suffit de montrer que $(K, F)$ est un Module induit libre de type fini. Puisque $C\left(\left(L^{\bullet}, F\right) \rightarrow(M, F)\right)$ est strict, on a

$$
G r^{F} K=\operatorname{Ker}\left(G r^{F} L^{-2 d_{X}+1} \rightarrow G r^{F} L^{-2 d_{X}+2}\right) \text {. }
$$

D'autre part, $\mathcal{E x t}_{G r \mathscr{D}}^{i}\left(G r^{F} K, N\right)={\mathcal{E} x t_{G r \mathscr{D}}^{i+2 d} x}\left(G r^{F} M, N\right)=0$ pour $i>0$ et pour tout $G r^{F} \mathscr{D}_{X}$-Module gradué $N$. On en conclut que $G r^{F} K$ est un facteur direct gradué de $G r^{F} L^{-2 d} x^{+1}$ (localement), donc par le lemme de Nakayama (gradué), $G r^{F} K$ (et donc $(K, F)$ ) est libre de type fini (quitte à se restreindre à un ouvert); d'où l'assertion. Ce qui achève la démonstration de 2.1.16.

2.1.18. Lemme. Soient $X$ une variété complexe lisse, et $U$ un sous-ouvert de Stein de $X$, tel que $\bar{U}$ soit compact. Soit $(M, F) \in M F\left(\mathscr{D}_{X}\right)$ tel que $\operatorname{Gr}^{F} M$ soit cohérent sur $G r^{F} \mathscr{D}_{X}$. On a alors $H^{i}(U, D R(M))=0$ pour $i>0$, où $D R(M)=$ $M \stackrel{L}{\otimes} \mathscr{D}_{\mathcal{O}}$.

Preuve. Soit $\left(L^{\bullet}, F\right) \rightarrow(M, F)$ la résolution canonique de 2.1.6, alors $G r^{F} G r_{p}^{G} L^{\bullet}$ sont acycliques sur $\bar{U}$ pour $p \gg 0$ d'après 2.1 .11 et 2.1 .17 , donc $\left.G_{p}\left(L^{\circ}, F\right)\right|_{U} \rightarrow$ $\left.(M, F)\right|_{U}$ est un quasi-isomorphisme filtré pour $\mathrm{p} \gg 0$. Puisque $D R\left(G_{p} L^{j}\right) \cong$ $G_{p} L^{j} \otimes \mathscr{D} \mathcal{O}$ sont des $\Theta_{X}$-Modules cohérents, l'assertion résulte du théorème $\mathrm{B}$ de Cartan.

2.1.19. Remarque. Ce lemme correspond à un résultat d'Artin, cf. [1].

2.1.20. Remarque. Si $X$ n'est pas lisse, on peut définir $D F\left(\mathscr{D}_{X}\right)$, etc. comme suit. On prend un recouvrement localement fini $X=\cup U_{i}$ avec des immer- 
sions fermées $U_{i} \rightarrow V_{i}$, où $V_{i}$ sont lisses. Posons $U_{I}=\bigcap_{i \in I} U_{i}$ et $V_{I}=\Pi_{i \in I} V_{i}$. Soient $i_{I}: U_{I} \rightarrow V_{I}$ les inclusions et $p r_{I J}: V_{J} \rightarrow V_{I}$ les projections $(I \subset J)$. Alors les objets de $M F\left(\mathscr{D}_{X}\right)$ sont les familles d'objets $\left(M_{I}, F\right)$ de $M F\left(\mathscr{D}_{V_{I}}\right)$ munis d'isomorphismes:

$$
u_{I J}:\left(p r_{I J}\right)_{*}\left(M_{J}, F\right) \cong\left(M_{I}, F\right) \text { sur } \quad V_{I} \backslash\left(U_{I} \backslash U_{J}\right),
$$

tels que chaque $G r_{p}^{F} M_{I}$ soit annulé par l'Idéal de $U_{I}$, et que $u_{I J}$ satisfassent les relations: $u_{I K}=u_{I J} \circ\left(p r_{I J}\right)_{*} u_{J K}$ sur $V_{I} \backslash\left(U_{I} \backslash U_{K}\right)$. (Notons que cette définition est indépendante du choix de $U_{i}, V_{i}$.) On définit $D F\left(\mathscr{D}_{X}\right)$, etc. comme dans 2.1.8, etc.

\subsection{Foncteur de de Rham et complexes filtrés différentiels}

2.2.1. Soit $M_{i}\left(\mathscr{D}_{X}\right)$ la sous-catégorie pleine de $M\left(\mathscr{D}_{X}\right)$ des Modules induits, i.e. les objets de $M_{i}\left(\mathscr{D}_{X}\right)$ sont les $\mathscr{D}_{X}$-Modules isomorphes à $L \otimes \mathscr{O}_{X} \mathscr{D}_{X}$ pour des $\mathscr{D}_{X}$-Modules $L$. On définit le foncteur de de Rham

$$
D R: M_{i}\left(\mathscr{D}_{X}\right) \rightarrow M\left(\boldsymbol{C}_{X}\right)
$$

par

$$
D R(M)=M \otimes_{\mathscr{D}} \mathcal{O}_{X},
$$

donc on a l'isomorphisme canonique: $D R(M)=L$ si $M=L \otimes_{\mathcal{O}} \mathscr{D}_{X}$. Ici $M(A)$ signifie la catégorie des A-Modules pour $M=\mathscr{D}_{X}, \mathcal{O}_{X}$ ou $\boldsymbol{C}_{X}$, etc.

2.2.2. Lemme. Soit $M_{j} \in M_{i}\left(\mathscr{D}_{X}\right)(j=1,2)$, alors le morphisme:

$$
D R: \operatorname{Ham}_{\mathscr{D}}\left(M_{1}, M_{2}\right) \rightarrow \operatorname{Ham}_{C}\left(D R\left(M_{1}\right), D R\left(M_{2}\right)\right)
$$

est injectif.

Preuve. On peut supposer que $M_{j}=L_{j} \otimes_{\mathcal{O}} \mathscr{D}_{X}$. Soit $\left(x_{1}, \cdots, x_{d_{X}}\right)$ un système de coordonnées locales de $X$. Si $\phi \in \operatorname{Ham}_{\mathcal{O}}\left(L_{1}, M_{2}\right)=\operatorname{Ham}_{\mathscr{D}}\left(M_{1}, M_{2}\right)$ n'est pas nul, il existe $m \in L_{1}$ tel que $\phi(m) \neq 0$. Posons

$$
\phi(m)=\Sigma_{\nu} u_{\nu} \otimes \partial^{\nu} \quad \text { avec } \quad u_{\nu} \in L_{2} \quad\left(\nu \in Z^{d} x\right),
$$

où $\partial^{\nu}=\Pi \partial_{i}^{\nu}$ avec $\partial_{i}=\partial / \partial x_{i}$. Soit

$$
i=\min \left\{|\nu|: u_{\nu} \neq 0\right\} \quad \text { où } \quad|\nu|=\sum \nu_{i},
$$

on prend $\mu \in \boldsymbol{Z}^{d} \boldsymbol{X}$ tel que $|\mu|=i$ et que $u_{\mu} \neq 0$, alors

$$
\phi\left(m x^{\mu}\right) \sum u_{\nu} \otimes \partial^{\nu} x^{\mu} \equiv \mu ! u_{\mu} \quad\left(\bmod . L_{2} \otimes\left(\sum \mathscr{D} \partial_{i}\right)\right)
$$

donc $(D R \phi)\left(m x^{\mu}\right)=\mu ! u_{\mu} \neq 0 ;$ d'où l'assertion. 
2.2.3. Soient $L_{1}$ et $L_{2}$ des $\mathcal{O}_{X}$-Modules, alors Hom $_{\text {Diff }}\left(L_{1}, L_{2}\right)$ le groupe des morphismes différentiels est l'image du monomorphisme (2.2.2.1) pour $M_{j}=$ $L_{j} \otimes \mathscr{D}_{X}$ (donc $\left.L_{j}=D R\left(M_{j}\right)\right)(j=1,2)$. Puisque Hom $\mathscr{D}\left(M_{1}, M_{2}\right)$ est muni de la filtration (pas nécessairement exhaustive):

$$
F_{p}=\operatorname{Ham} \Theta\left(L_{1}, L_{2} \otimes F_{p} \mathscr{D}_{X}\right)
$$

Ham $_{\text {Diff }}\left(L_{1}, L_{2}\right)$ est muni de la filtration $F$ par le foncteur $D R$. On dit qu'un morphisme différentiel est d'ordre $\leq p$ s'il appartient à $F_{p}$ Hom $_{\text {Diff }}\left(L_{1}, L_{2}\right)$.

2.2.4. Soit $\left(L_{j}, F\right)$ un $\mathcal{O}_{X}$-Module filtré tel que $F_{p} L_{j}=0$ pour $p \ll 0$ localement sur $X(j=1,2)$, alors Ham $_{\text {Diff }}\left(\left(L_{1}, F\right),\left(L_{2}, F\right)\right)$ le groupe des morphismes différentiels filtrés est le sous-groupe de $\operatorname{Ham}_{\text {Diff }}\left(L_{1}, L_{2}\right)$, qui consiste en les morphismes $\phi$ satisfaisant la condition:

(2.2.4.1) la composition $F_{p} L_{1} \rightarrow L_{1} \stackrel{\phi}{\rightarrow} L_{2} \rightarrow L_{2} / F_{p-q-1} L_{2}$ est d'ordre $\leq q$ pour tout $p, q$.

Notons que cette condition entraîne que $\phi\left(F_{p} L_{1}\right) \subset F_{p} L_{2}$ (pour $q=-1$ ) et que $G r^{F} \phi: G r_{p}^{F} L_{1} \rightarrow G r_{p}^{F} L_{2}$ est $\mathcal{O}_{X}$-linéaire (pour $q=0$ ).

Soit $M F\left(\mathcal{O}_{X}\right.$, Diff $)$ la catégorie additive dont les objets sont les $\mathcal{O}_{X}$-Modules filtrés $(L, F)$ tels que $F_{p} L=0$ pour $p \ll 0$ localement sur $X$, et dont les morphismes sont les morphismes différentiels filtrés. Soit $\operatorname{MF}^{f}\left(\mathcal{O}_{X}\right.$, Diff $)$ la sous-catégorie pleine de $M F\left(\mathcal{O}_{X}\right.$, Diff $)$ des objets satisfaisant la condition:

$$
F_{p} L=L \text { pour } p \gg 0 \text { localement sur } X \text {. }
$$

2.2.5. Lemme. Soient $\left(L_{j}, F\right) \in M F\left(\mathcal{O}_{X}\right.$, Diff $)$ et $\left(M_{j}, F\right)=\left(L_{j}, F\right) \otimes$ $\left(\mathscr{D}_{X}, F\right)(j=1,2)$, alors le foncteur $D R$ induit l'isomorphisme:

$$
D R: \operatorname{Ham}_{\mathscr{D}}\left(\left(M_{1}, F\right),\left(M_{2}, F\right)\right) \underset{\rightarrow}{\rightarrow} \operatorname{Ham}_{\text {Diff }}\left(\left(L_{1}, F\right),\left(L_{2}, F\right)\right) \text {. }
$$

Preuve. Soient $\phi \in \operatorname{Ham}_{\mathrm{Diff}}\left(L_{1}, L_{2}\right)$ et $\psi \in \operatorname{Ham}_{\mathcal{D}}\left(M_{1}, M_{2}\right)$ tels que $\phi=D R(\psi)$, alors il suffit de vérifier que

$$
\phi \in \operatorname{Ham}_{\mathrm{Diff}}\left(\left(L_{1}, F\right),\left(L_{2}, F\right)\right) \quad \text { sii } \quad \psi\left(F_{i} L_{1}\right) \subset \Sigma_{j} F_{i-j} L_{2} \otimes F_{j} \mathscr{D}_{X} \quad \text { pour tout } i \text {. }
$$

Mais la première condition équivaut à

$$
\psi\left(F_{i} L_{1}\right) \subset L_{2} \otimes F_{j} \mathscr{D}_{X}+F_{i-j-1} L_{2} \otimes \mathscr{D}_{X} \quad \text { pour tout } i, j,
$$

cf. (2.2.4.1); d'où l'assertion.

2.2.6. Corollaire. On a l'équivalence de catégories: 


$$
D R^{-1}: M F^{(f)}\left(\mathcal{O}_{X}, \text { Diff }\right) \underset{\rightarrow}{\longrightarrow} M F_{i}^{(f)}\left(\mathscr{D}_{X}\right)
$$

telle que $D R^{-1}(L, F)=(L, F) \otimes\left(\mathscr{D}_{X}, F\right)$ et que

$$
D R^{-1}: \operatorname{Ham}_{\text {Diff }}\left(\left(L_{1}, F\right),\left(L_{2}, F\right)\right) \rightarrow\left(\operatorname{Hom}_{\mathscr{D}}\left(D R^{-1}\left(L_{1}, F\right), D R^{-1}\left(L_{2}, F\right)\right)\right.
$$

est l'inverse de (2.2.5.1).

Remarque. On a un quasi-inverse $D R$ de $D R^{-1}$, si on choisit un isomorphisme $(M, F) \simeq(L, F) \otimes\left(\mathscr{D}_{X}, F\right)$ pour tout $(M, F) \in M F_{i}\left(\mathscr{D}_{X}\right)$.

2.2.7. Soit $\left(\mathscr{D}_{X} \otimes_{\mathcal{O}} \Lambda^{-*} \Theta_{X}, F\right) \rightarrow\left(\mathcal{O}_{X}, F\right)$ comme dans 2.1.5. Soit $(M, F) \in$ $\operatorname{MF}\left(\mathscr{D}_{X}\right)$. Posons

$$
\widetilde{D R}(M, F)=(M, F) \otimes_{\mathscr{D}}\left(\mathscr{D}_{X} \otimes_{\mathcal{O}} \Lambda^{\Lambda^{-} \Theta_{X}}, F\right) .
$$

Alors par l'isomorphisme canonique:

$$
(\widetilde{D R}(M, F))^{i}=(M, F) \otimes_{\mathcal{O}}\left(\Lambda^{-i} \Theta_{X}, F\right),
$$

(où la filtration $F$ sur $A^{-i} \Theta_{X}$ est définie par $G r_{p}^{F}=0$ pour $\left.p \neq-i\right),(\widetilde{D R}(M, F))^{i}$ est muni d'une structure de $\mathcal{O}_{X}$-Module, donc c'est un objet de $M F\left(\mathcal{O}_{X}\right.$, Diff). Notons que $\widetilde{D R}(M)$ est localement isomorphe au complexe de Koszul

$$
K\left(M ; \partial_{i}\left(1 \leq i \leq d_{X}\right)\right)\left[d_{X}\right] .
$$

2.2.8. Lemme. Avec les notations ci-dessus, $\widetilde{D R}(M, F)$ est un complexe de $M F\left(\mathcal{O}_{X}\right.$, Diff) (par la structure de $\mathcal{O}_{X}$-Module définie ci-dessus), tel que $D R^{-1}(\widetilde{D R}(M, F))$ est canoniquement isomorphe au premier complexe de (2.1.6.1).

Preuve. Soient les notations comme dans la preuve de 2.1.6, e.g. $L=\Lambda^{-i} \Theta_{X}$, alors par l'isomorphisme (2.1.6.2), $(M, F) \otimes \mathcal{O}_{\mathcal{O}}\left(\mathscr{D}_{X} \otimes L, F[-i]\right)$ est un Module induit dont l'image par le foncteur $D R$ est munie d'une structure de $\mathcal{O}_{X}$-Module et isomorphe à $(M, F) \otimes_{\mathcal{O}}(L, F[-i])$. On vérifie facilement que la différentielle de $\widetilde{D R}(M)$ coïncide avec celle de l'image du premier complexe de (2.1.6.1) par le foncteur $D R$ (par les isomorphismes ci-dessus), car ces deux complexs sont localement isomorphes au complexe de $\operatorname{Koszul} K\left(M ; \partial_{i}\left(1 \leq i \leq d_{X}\right)\right)\left[d_{x}\right]$ si on choisit un système de coordonnées locales (l'assertion pour le deuxèime complexe résulte aussi de 2.4.2); ce qui achève la démonstration.

2.2.9. Soit $\hat{\imath}=b,+,-$ ou $\infty$. On définit

$$
\begin{aligned}
& C^{\text {म }} F^{(f)}\left(\mathcal{O}_{X}, \text { Diff }\right), K^{\text {ये }} F^{(f)}\left(\mathcal{O}_{X}, \text { Diff }\right), \\
& D^{\text {मे }} F^{(f)}\left(\mathcal{O}_{X}, \text { Diff }\right), D F^{(f)}\left(\mathcal{O}_{X}, \text { Diff }\right)^{\text {मे }} \text {, }
\end{aligned}
$$


comme dans 2.1.9, en remplaçant $M F\left(\mathscr{D}_{X}\right)$ par $M F\left(\mathcal{O}_{X}\right.$, Diff) (ou $M F^{f}\left(\mathcal{O}_{X}\right.$, Diff)). Notons que

$$
\mathscr{H}^{\bullet} G r_{i}^{F}: K^{\text {is }} F^{(f)}\left(\mathcal{O}_{X}, \text { Diff }\right) \rightarrow M\left(\mathcal{O}_{X}\right)
$$

sont des foncteurs cohomologiques. Soit $D_{\text {coh }}^{\text {茨 }} F^{f}\left(\Theta_{X}\right.$, Diff $)$ la sous-catégorie pleine de $D^{\text {is }} F^{f}\left(\Theta_{X}\right.$, Diff) des objets cohomologiquement cohérents (i.e. l'image par $\mathcal{H}^{j} G r_{i}^{F}$ est un $\mathcal{O}_{X}$-Module cohérent pour tout $i, j$.) Notons que

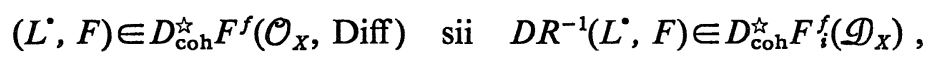

cf. 2.1.15.

2.2.10. Proposition. Avec les notations de 2.1.9, 2.1.10, 2.1.15 et 2.2.9, le foncteur $D R^{-1}$ induit des équivalences de catégories:

(2.2.10.1) $\quad D^{\text {ఓ }} F\left(\mathcal{O}_{X}\right.$, Diff $) \simeq D^{\natural} F\left(\mathscr{D}_{X}\right)$

(2.2.10.2) $D F\left(\mathcal{O}_{X}, \text { Diff }\right)^{\text {और }} \simeq D F\left(\mathscr{D}_{X}\right)^{\text {出 }}$

(2.2.10.3) $\quad D^{\natural} F^{f}\left(\mathcal{O}_{X}\right.$, Diff $) \simeq D^{\natural} F_{i}^{f}\left(\mathscr{D}_{X}\right)$

(2.2.10.4) $D F^{f}\left(\mathcal{O}_{X}, \text { Diff }\right)^{\natural} \simeq D F_{i}^{f}\left(\mathscr{D}_{X}\right)$

(2.2.10.5) $\quad D_{\mathrm{coh}}^{b} F^{f}\left(\mathcal{O}_{X}\right.$, Diff $) \simeq D_{\mathrm{coh}}^{b} F\left(\mathscr{D}_{X}\right)$

avec $\widetilde{D R}$ pour quasi-inverse.

Preuve. D'après 2.1 .6 et $\mathbf{2 . 2 . 8}$, on a le quasi-isomorphisme filtré canonique:

(2.2.10.6) $D R^{-1}\left(\widetilde{D R}\left(M^{*}, F\right)\right) \underset{\rightarrow}{\rightarrow}\left(M^{*}, F\right)$ pour $\left(M^{*}, F\right) \in K F\left(\mathscr{D}_{X}\right)$.

En l'appliquant à $\left(M^{*}, F\right)=D R^{-1}\left(L^{*}, F\right)$ pour $\left(L^{*}, F\right) \in K F\left(\mathcal{O}_{X}\right.$, Diff $)$, on obtient le morphimse canonique dans $K F\left(\mathcal{O}_{X}\right.$, Diff):

(2.2.10.7) $\tilde{D R}\left(D R^{-1}\left(L^{*}, F\right)\right) \rightarrow\left(L^{*}, F\right)$

par la définition de $D R^{-1}$. On vérifie facilement que ce morphisme est un quasi-isomorphisme filtré. (On peut aussi utiliser le lemme 2.1.11.) Puisque

$$
\begin{aligned}
& D R^{-1}\left(K F\left(\mathcal{O}_{X}, \text { Diff }\right)^{*}\right) \subset K F\left(\mathscr{D}_{X}\right)^{*} \\
& \widetilde{D R}\left(K F\left(\mathscr{D}_{X}\right)^{*}\right) \subset K F\left(\mathcal{O}_{X}, \text { Diff }\right)^{*}
\end{aligned}
$$

on obtient les assertions (2.2.10.1-4). La dernière résulte de 2.1.16.

2.2.11. Remarque. On peut définir (comme Du Bois, Bull. Soc. math. France, 109) les catégories:

$$
K^{\natural} F_{1}\left(\Theta_{X}, \text { Diff }\right) \text { et } D^{\natural} F_{1}\left(\Theta_{X}, \text { Diff }\right), \text { etc. }
$$


en supposant que les différentielles (resp. les morphismes et les homotopies) de complexes sont d'ordre $\leq 1$ (resp. 0); alors le foncteur $\widetilde{D R}$ se factorise par $D^{\text {ג }} F_{1}\left(\mathcal{O}_{X}\right.$, Diff $) \rightarrow D^{\text {h }} F\left(\mathcal{O}_{X}\right.$, Diff $)$, mais le morphisme (2.2.10.7) n'est pas défini dans $K^{\text {ฟे }} F_{1}\left(\mathcal{O}_{X}\right.$, Diff $)$. Je ne sais si le foncteur naturel:

$$
D^{\Uparrow} F_{1}\left(\mathcal{O}_{X}, \text { Diff }\right) \rightarrow D^{\star} F\left(\mathcal{O}_{X}, \text { Diff }\right)
$$

est fidèle.

\subsection{Foncteur image directe}

2.3.1. Soit $f: X \rightarrow Y$ un morphisme de variétés complexes lisses. On définit les catégories:

$$
M F\left(\mathscr{D}_{X}\right)_{f}, D^{\natural} F\left(\mathscr{D}_{X}\right)_{f}, D^{\natural} F_{i}^{(f)}\left(\mathscr{D}_{X}\right)_{f}, \text { etc. }
$$

et

$$
M F\left(\mathcal{O}_{X}, \text { Diff }\right)_{f}, D^{\text {i }} F\left(\mathcal{O}_{X}, \text { Diff }\right)_{f} \text {, etc. }
$$

comme dans 2.1 et 2.2, en remplaçant la condition: "localement sur $X$ " par "localement rel. à $Y$ ". Donc $M F\left(\mathscr{D}_{X}\right)_{f}=M F\left(\mathscr{D}_{X}\right)$ etc., si $f$ est propre. En général on peut vérifier que $D F\left(\mathscr{D}_{X}\right)_{f}$ est une sous-catégorie pleine de $D F\left(\mathscr{D}_{X}\right)$ par un argument dual de la preuve de 2.1 .12 (i.e. on utilise $\left(j_{I}\right) * j_{I}^{-1}\left(M_{2}^{*} /\right.$ $\left.F_{p(I)} M_{2}^{*}, F\right)$ avec $p(I)=\max \left\{p: j_{I}^{-1} F_{p} M_{1}=0\right\}$, où $\left.U_{I}=f^{-1} f\left(U_{I}\right)\right)$. Puisque tous les résultats de 2.1 et de 2.2 (sauf 2.1.16 et (2.2.10.5)) restent valable en

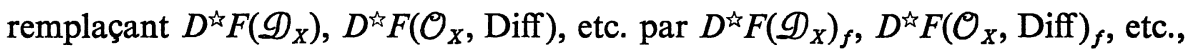
on voit que $D^{\natural} F\left(\mathscr{D}_{X}\right)_{f}, D^{\text {丸 }} F_{i}^{(f)}\left(\mathscr{D}_{X}\right)_{f}$, etc. (resp. $D^{\natural} F\left(\mathcal{O}_{X}, \text { Diff }\right)_{f}$, etc.) sont des sous-catégories pleines de $D F\left(\mathscr{D}_{X}\right)$ (resp. $D F\left(\mathcal{O}_{X}\right.$ Diff)).

2.3.2. Avec les notations de 2.3.1, on définit $D^{\not 4} F\left(f^{-1} \mathscr{D}_{Y}\right)_{f}, D^{\not 4} F_{i}^{(f)}\left(f^{-1} \mathscr{D}_{Y}\right)_{f}$, etc. comme ci-dessus, en remplaçant respectivement $\mathscr{D}_{X}$ et $\mathcal{O}_{X}$ par $f^{-1} \mathscr{D}_{Y}$ et $f^{-1} \mathcal{O}_{Y}$.

Soit $\left(\mathscr{D}_{X \rightarrow Y}, F\right)=\mathcal{O}_{X} \otimes_{f^{-1} \mathcal{O}_{Y}} f^{-1}\left(\mathscr{D}_{Y}, F\right)$. On définit le foncteur $D R_{X / Y}$ : $M F_{i}^{(f)}\left(\mathscr{D}_{X}\right)_{f} \rightarrow M F_{i}^{(f)}\left(f^{-1} \mathscr{D}_{Y}\right)_{f}$ par

$$
D R_{X / Y}(M, F)=(M, F) \otimes_{\mathscr{D}}\left(\mathscr{D}_{X \rightarrow Y}, F\right) .
$$

Donc, si $(M, F) \simeq(L, F) \otimes\left(\mathscr{D}_{X}, F\right)$, on a

$$
\begin{aligned}
D R_{X / Y}(M, F): & =(M, F) \otimes_{\mathscr{D}}\left(\mathscr{D}_{X \rightarrow Y}, F\right) \\
& \simeq(L, F) \otimes_{f^{-1} \mathcal{O}_{Y}}\left(f^{-1} \mathscr{D}_{Y}, F\right) .
\end{aligned}
$$

Le foncteur $D R_{X / Y}$ induit les foncteurs:

$$
D R_{X / Y}: K F_{i}^{(f)}\left(\mathscr{D}_{X}\right)_{f} \rightarrow K F_{i}^{(f)}\left(f^{-1} \mathscr{D}_{Y}\right)_{f}
$$


et

$$
D R_{X / Y}: D F_{i}^{(f)}\left(\mathscr{D}_{X}\right)_{f} \rightarrow D F_{i}^{(f)}\left(f^{-1} \mathscr{D}_{Y}\right)_{f}
$$

d'après 2.1.11.

2.3.3. Avec les notations ci-dessus, soit $(M, F)$ un faisceau filtré sur $X$ tel que $\bigcup_{p} F_{p} M=M$. On définit son image directe topologique par $f_{*}(M, F)=$ $\left(\bigcup_{p} f_{*} F_{p} M, F\right)$ avec $F_{p}\left(f_{*}(M, F)\right)=f_{*} F_{p} M$. Alors ce foncteur image directe topologique filtrée $f_{*}$ induit le foncteur:

$$
f_{*}: M F_{i}^{(f)}\left(f^{-1} \mathscr{D}_{Y}\right)_{f} \rightarrow M F_{i}^{(f)}\left(\mathscr{D}_{Y}\right)
$$

Puisque la dimension topologique stricte de $X$ est finie [33], ce foncteur $f_{*}$ se dérive en

$$
f_{*}: D^{*} F_{i}^{(f)}\left(f^{-1} \mathscr{D}_{Y}\right)_{f} \rightarrow D^{*} F_{i}^{(f)}\left(\mathscr{D}_{Y}\right)
$$

d'après le lemme suivant:

2.3.4. Lemme. Soit $g^{0}$ le foncteur exact qui associe le faisceau des sections discontinues. Soit $(M, F)=(L, F) \otimes\left(f^{-1} \mathscr{D}_{Y}, F\right) \in M F_{i}^{(f)}\left(f^{-1} \mathscr{D}_{Y}\right)$. Alors le morphisme canonique

$$
\left(g^{0}(L, F)\right) \otimes\left(f^{-1} \mathscr{D}_{Y}, F\right) \rightarrow \mathscr{g}^{0}\left((L, F) \otimes\left(f^{-1} \mathscr{D}_{Y}, F\right)\right)
$$

est un isomorphisme filtre, ou $g^{0}(M, F)=\left(\bigcup_{p} g^{0} F_{p} M, F\right)$ pour un faisceau filtré $(M, F)$.

\section{(C'est clair.)}

Remarque. Pour définir le foncteur dérivé $f_{*}$, il suffit de construire une résolution finie, canonique et fonctorielle: $(M, F) \rightarrow\left(L^{*}, F\right)$ pour $(M, F) \in$ $M F_{i}^{(f)}\left(f^{-1} \mathscr{D}_{Y}\right)_{f}$, telle que chaque $G r_{p}^{F} L^{j}$. soit $f_{*}$-acyclique (mais $L^{j}$ n'est pas nécessairement $f_{*}$-acyclique.) De même, on peut définir le foncteur $f_{!}$.

2.3.5. On définit le foncteur image directe $f_{*}$ par la composition:

$$
D F\left(\mathscr{D}_{X}\right)_{f} \stackrel{D R^{-} \circ \widetilde{D R}}{\longrightarrow} D F_{i}\left(\mathscr{D}_{X}\right)_{f} \stackrel{f_{*}}{\rightarrow} D F_{i}\left(\mathscr{D}_{Y}\right) \stackrel{\sim}{\rightarrow} D F\left(\mathscr{D}_{Y}\right) .
$$

Si $f$ est propre ce foncteur induit:

$$
f_{*}: D_{\mathrm{coh}}^{b} F\left(\mathscr{D}_{X}\right) \rightarrow D_{\mathrm{coh}}^{b} F\left(\mathscr{D}_{Y}\right)
$$

d'après 2.1.16 et par le théorème de Grauert.

2.3.6. Lemme. Avec les notations de 2.3.1, le foncteur limage directe 
topologique filtrée $f_{*}$ (cf. 2.3.3) induit le foncteur:

$$
f_{*}: M F^{(f)}\left(\Theta_{X}, \text { Diff }\right)_{f} \rightarrow M F^{(f)}\left(\Theta_{Y}, \text { Diff }\right)
$$

(i.e. les morphismes differentiels filtrés sont stables par image directe topologique), de plus le diagramme suivant commute:

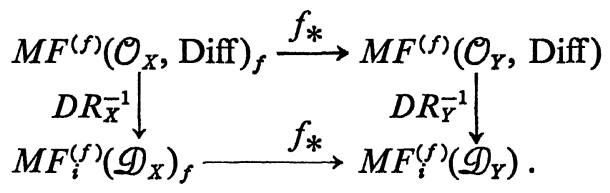

Preuve. Par définition, on a l'isomorphisme canonique:

$$
f_{*}\left(D R_{\bar{X}}^{-1}(L, F)\right) \simeq D R_{\bar{Y}}^{-1}\left(f_{*}(L, F)\right)
$$

pour $(L, F) \in M F^{(f)}\left(\mathcal{O}_{X} \text {, Diff }\right)_{f}$, donc par la définition de $D R_{Y}^{-1}$, il suffit de vérifier que, pour $\phi:\left(L_{1}, F\right) \rightarrow\left(L_{2}, F\right)$ un morphisme de $M F^{(f)}\left(\mathcal{O}_{X}, \text { Diff }\right)_{f}$, on a

$$
D R_{Y}\left(f_{*}\left(D R_{X}^{-1}(\phi)\right)\right)=f_{*}(\phi): f_{*}\left(L_{1}, F\right) \rightarrow f_{*}\left(L_{2}, F\right) .
$$

Soit $\psi=D R_{X}^{-1}(\phi)$, alors

$$
D R_{Y}\left(f_{*}(\psi)\right)=D R_{Y}\left(f_{*}\left(D R_{X / Y}(\psi)\right)\right)=f_{*}\left(f^{-1} D R_{Y}\left(D R_{X / Y}(\psi)\right)\right),
$$

où $f^{-1} D R_{Y}$ est le foncteur défini par $\otimes_{f^{-1} \mathscr{D}_{Y}} f^{-1} \mathcal{O}_{Y}$; donc il suffit de vérifier que

$$
f^{-1} D R_{Y}\left(D R_{X / Y}(\psi)\right)=D R_{X}(\psi):\left(L_{1}, F\right) \rightarrow\left(L_{2}, F\right) .
$$

Soit $1_{X \rightarrow Y}:=1 \otimes 1 \in \mathscr{D}_{X \rightarrow Y}=\mathcal{O}_{X} \otimes_{f^{-1} \mathcal{O}_{Y}} f^{-1} \mathscr{D}_{Y}$. On a des décompositions canoniques:

$$
\begin{aligned}
& L_{2} \otimes_{\mathcal{O}_{X}} \mathscr{D}_{X}=\left(L_{2} \otimes 1\right) \oplus\left(L_{2} \otimes \mathscr{D}_{X} \Theta_{X}\right) \\
& L_{2} \otimes_{f^{-1} \mathcal{O}_{Y}} f^{-1} \mathscr{D}_{Y}=\left(L_{2} \otimes 1\right) \oplus\left(L_{2} \otimes f^{-1} \mathscr{D}_{Y} \Theta_{Y}\right)
\end{aligned}
$$

telles que $\left(L_{2} \otimes 1\right) \otimes 1_{X \rightarrow Y}=L_{2} \otimes 1$ et que $\left(L_{2} \otimes \mathcal{D}_{X} \Theta_{X}\right) \otimes 1_{X \rightarrow Y} \subset L_{2} \otimes f^{-1} \mathscr{D}_{Y} \Theta_{Y}$ par l'isomorphisme canonique:

$$
\left(L_{2} \otimes_{\mathcal{O}_{X}} \mathscr{D}_{X}\right) \otimes_{\mathscr{D}_{X}} \mathscr{D}_{X \rightarrow Y}=L_{2} \otimes_{f^{-1} \mathcal{O}_{Y}} f^{-1} \mathscr{D}_{Y}
$$

ce qui entraîne l'assertion.

2.3.7. Avec les notations ci-dessus, les foncteurs $g^{0}$ et $g^{0} /$ id induisent les foncteurs de $M F^{(f)}\left(\mathcal{O}_{X} \text {, Diff }\right)_{f}$ dans elle-même d'après 2.3 .4 , et on obtient une résolution finie, canonique et fonctorielle: $(M, F) \rightarrow\left(L^{*}, F\right)$ telle que chaque $G r_{p}^{F} L^{j}$ soit $f_{*}$-acyclique; donc le foncteur image directe topologique 
filtrée $f_{*}$ se dérive en

$$
\begin{array}{ll}
f_{*}: D^{\natural} F^{(f)}\left(\mathcal{O}_{X}, \text { Diff }\right)_{f} \rightarrow D^{\natural} F^{(f)}\left(\mathcal{O}_{Y}, \text { Diff }\right) \\
\text { (et } \left.\quad f_{*}: D_{\text {coh }}^{\natural} F^{f}\left(\mathcal{O}_{X}, \text { Diff }\right) \rightarrow D_{\text {coh }}^{\varkappa} F^{f}\left(\mathcal{O}_{X}, \text { Diff }\right) \text { si } f \text { est propre }\right)
\end{array}
$$

de sorte que $D R_{Y}^{-1} \circ f_{*}=f_{*} \circ D R_{X}^{-1}$, cf. 2.3.6. Notons que $f_{*}$ commute au foncteur naturel: $D F\left(\mathcal{O}_{X}\right.$, Diff $) \rightarrow D\left(C_{X}\right)$, si $f$ est propre.

Pour $f: X \rightarrow Y$ et $g: Y \rightarrow Z$, on a canoniquement $(g f)_{*}=g_{*} f_{*}$ par définition, donc c'est valable pour les images directes de complexes de $\mathscr{D}$-Modules filtrés.

\subsubsection{Remarques.}

(1) Pour un complexe de $\mathscr{D}_{X}$-Modules $M^{*}$ on définit usuellement:

$$
\int_{f} M^{*}=\boldsymbol{R} f_{*}\left(M^{*} \otimes_{\mathscr{D}_{X}}^{\boldsymbol{L}} \mathcal{D}_{X \rightarrow Y}\right),
$$

mais on n'a pas en général $\int_{g f}=\int_{g} \int_{f}$, e.g. posons $X=C, Y=\{0\}, Z=C$, $\left\{a_{i}\right\}_{i \in N} \subset X$ avec $\left|a_{i}\right| \rightarrow \infty, M=\bigoplus_{i} \mathscr{B}_{\left(a_{i}\right) \mid X}$ avec $\mathscr{B}_{\left(a_{i}\right) \mid X}=\mathscr{D}_{X} /\left(x-a_{i}\right) \mathscr{D}_{X}$, où $x$ est la coordonnée de $X$.

En particulier, en oubliant la filtration, $f_{*}\left(M^{*}, F\right)$ ne coïncide pas avec $\int_{f} M^{\bullet}$. Mais ils coincident, si la condition suivante est vérifiée:

Il existe un sous-ensemble $Z$ de $X$, propre sur $Y$, tel que

$\left.G_{p}^{Y} D R_{X / Y}\left(M^{\bullet}, F\right)\right|_{X \backslash Z}$ soit acyclique pour $p \gg 0$ localement rel. à $Y$. Par exemple, soit $M=\Omega_{X}^{d} x$ avec $G r_{p}^{F} M=0$ pour $p \neq-d_{X}$, alors, si $f$ est lisse, $D R_{X / Y}(M, F) \simeq\left(\Omega_{X / Y}^{\circ}, F\right)\left[d_{X}-d_{Y}\right]$ localement rel. à $Y$; donc la condition cidessus est vérifiée, si $\operatorname{Sing} f$ est propre.

(2) Le théorème $B$ de Cartan pour les $\mathscr{D}$-Modules n'est pas vrai en général, car $H^{1}\left(X, \stackrel{N}{\oplus} \mathcal{O}_{X}\right) \neq 0$ pour $X=C$ (ou $X$ est un polydisque). En effet, pour $\left\{a_{i}\right\}$ comme ci-dessus, considérons la suite exacte longue associée à:

$$
0 \rightarrow \stackrel{N}{\oplus} \mathcal{O}_{X} \rightarrow \stackrel{N}{\oplus} \mathcal{O}_{X} \rightarrow \stackrel{N}{\oplus} C_{a_{i}} \rightarrow 0
$$

2.3.9. Remarque. Soit $f: X \rightarrow Y$ un morphisme propre de variétés analytiques complexes (séparées), alors on peut définir $f_{*}: D F\left(\mathscr{D}_{X}\right) \rightarrow D F\left(\mathscr{D}_{Y}\right)$, etc., en utilisant un complexe de Čech associé aux recouvrements $X=\cup U_{i}, Y=\cup U_{i}^{\prime}$ avec des immersions $U_{i} \rightarrow V_{i}, U_{i}^{\prime} \rightarrow V_{i}^{\prime}$ comme dans 2.1.20, tels qu'on ait des diagrammes commutatifs:

$$
\begin{aligned}
U_{i} & \rightarrow V_{i} \\
f \downarrow & \downarrow f_{i} \\
U_{i}^{\prime} & \rightarrow V_{i}^{\prime}
\end{aligned}
$$


On peut vérifier que $f_{*}$ ne dépend pas de choix de $U_{i}, V_{i}, U_{i}^{\prime}, V_{i}^{\prime}$ et de $f_{i}$.

\subsection{Foncteur dual}

2.4.1. Soit $M$ un $\mathscr{D}_{X}$-Module à droite, on a alors deux structures de $\mathscr{D}_{X^{-}}$ Module à droite, notées $\boldsymbol{r}$ et $\boldsymbol{t}$, sur $M \otimes_{\mathcal{O}} \mathscr{D}_{X}$, définies par:

$$
\begin{array}{ll}
(m \otimes P) r(g)=m \otimes P g, & (m \otimes P) r\left(\partial_{i}\right)=m \otimes P \partial_{i} \\
(m \otimes P) t(g)=m g \otimes P, & (m \otimes P) t\left(\partial_{i}\right)=m \partial_{i} \otimes P-m \otimes \partial_{i} P
\end{array}
$$

pour $m \in M, P \in \mathscr{D}_{X}, g \in \mathcal{O}_{X}$ et $\partial_{i}=\partial / \partial x_{i}$

2.4.2. Lemme. Soit $M$ un $\mathscr{D}_{X}$-Module à droite, alors il existe une unique involution ı: $M \otimes \mathcal{O}_{X} \rightarrow M \otimes \mathcal{O} \mathscr{D}_{X}$ identique sur $M \otimes 1$, qui échange les deux structures de $\mathscr{D}$-Module à droite.

Preuve. On définit le morphisme $\iota: M \otimes \mathscr{D}_{X} \rightarrow M \otimes \mathscr{D}_{X}$ par

$$
\iota(m \otimes P)=(m \otimes 1) t(P),
$$

alors il suffit de vérifier que $\iota^{2}=$ id. Soit $\partial^{\nu}=\Pi \partial_{i}^{\nu}$ pour $\nu \in \boldsymbol{N}^{d} \boldsymbol{x}$, posons $C(\mu, \nu)=\Pi_{i}\left(\mu_{i}+\nu_{i}\right) ! / \mu_{i} ! \cdot \nu_{i} !$ pour $\mu, \nu \in \boldsymbol{N}^{d} x_{\text {. }}$ On a alors

$$
\begin{aligned}
\iota^{2}\left(m \otimes \partial^{\nu}\right) & =\imath\left(\sum_{0 \leq \mu \leq \nu}(-1)^{|\mu|} C(\mu, \nu-\mu) m \partial^{\nu-\mu} \otimes \partial^{\mu}\right) \\
& =\sum_{0 \leq \lambda \leq \mu \leq \nu}(-1)^{\left|\mu_{\mid}+\right| \lambda \mid} C(\mu, \nu-\mu) C(\lambda, \mu-\lambda) m \partial^{\nu-\lambda} \otimes \partial^{\lambda} \\
& =m \otimes \partial^{\nu}
\end{aligned}
$$

car $C(\mu, \nu-\mu) C(\lambda, \mu-\lambda)=C(\nu-\mu, \mu-\lambda) C(\lambda, \nu-\lambda)$; d'où l'assertion.

2.4.3. Soient $(M, F)$ et $\left(M^{\prime}, F\right)$ des $\mathscr{D}_{X}$-Modules filtrés, on définit le faisceau filtré $\operatorname{Ham}_{\mathscr{D}}^{F}\left((M, F),\left(M^{\prime}, F\right)\right)$ dont la filtration $F$ est exhaustive, par

$$
F_{p}:=\left\{\phi \in \operatorname{Ham}_{\mathcal{D}}\left(M, M^{\prime}\right): \phi\left(F_{i} M\right) \subset F_{p+i} M^{\prime} \text { pour tout } i\right\} .
$$

(De même, pour des $\mathcal{O}_{X}$-Modules filtrés.)

Soit $\omega_{X}\left[d_{X}\right] \rightarrow K_{X}^{*}$ une résolution par des $\mathscr{D}_{X}$-Modules, injectifs sur $\mathcal{O}_{X}$, telle que $K_{X}^{i}=0$ pour $i<-d_{X}$, où $\omega_{X}=\Omega_{X}^{d} x\left(d_{X}\right)$, cf. (2.0.1-2). Alors $K_{X} \otimes_{\mathcal{O}}$ $\left(\mathscr{D}_{X}, F\right)$ est un complexe de $\mathscr{D}_{X}$-bi-Modules à droite filtrés, donc pour $\left(M^{\bullet}, F\right)$ $\in C^{-} F_{i}^{f}\left(\mathscr{D}_{X}\right)$, on définit

$$
\boldsymbol{D}\left(M^{*}, F\right):=\operatorname{Ham}_{\mathcal{D}}^{F}\left(\left(M^{*}, F\right), K_{X}^{*} \otimes \mathcal{O}\left(\mathscr{D}_{X}, F\right)\right)
$$

où on peut utiliser au choix $\boldsymbol{r}$ ou $\boldsymbol{t}$ pour la structure de complexe de $\mathscr{D}_{X^{-}}$ Modules filtrés sur $\boldsymbol{D}\left(M^{\circ}, F\right)$ et l'autre pour $\operatorname{Ham}_{\mathscr{D}}^{F}$ d'après 2.4.2. 
Supposons que $(M, F)=(L, F) \otimes\left(\mathscr{D}_{X}, F\right)$ avec $(L, F) \in M F^{f}\left(\mathcal{O}_{X}\right.$, Diff $)$, on a alors

$$
(D(M, F))^{i}=\operatorname{Ham}_{\mathcal{O}}\left((L, F),\left(K_{X}^{i}, F\right)\right) \otimes_{\mathcal{O}}\left(\mathscr{D}_{X}, F\right)
$$

si on utilise $t$ pour $\operatorname{Ham}_{\mathcal{G}}^{F}$. (Ici la filtration $F$ de $K_{X}^{i}$ est triviale, i.e. $G r_{j}^{F} K_{X}^{i}=0$ pour $j \neq 0$.) On a donc $\boldsymbol{D}\left(M^{\bullet}, F\right) \in C^{+} F_{i}^{f}\left(\mathscr{D}_{X}\right)$ pour $\left(M^{\bullet}, F\right) \in C^{-} F_{i}^{f}\left(\mathscr{D}_{X}\right)$.

2.4.4. Lemme. Le foncteur dual $\boldsymbol{D}$ défini ci-dessus induit les foncteurs contravariants:

$$
\begin{aligned}
& \text { D: } K^{-} F_{i}^{f_{i}}\left(\mathscr{D}_{X}\right) \rightarrow K^{+} F_{i}^{f_{i}}\left(\mathscr{D}_{X}\right) \\
& \boldsymbol{D}: D^{-} F_{i}^{f}\left(\mathscr{D}_{X}\right) \rightarrow D^{+} F_{i}^{f}\left(\mathscr{D}_{X}\right)
\end{aligned}
$$

(e.g. $\boldsymbol{D}\left(M^{*}, F\right)$ est acyclique filtré, si $\left(M^{\circ}, F\right)$ l'est $)$. Le dernier foncteur est indépendant du choix de $K_{\dot{X}}^{\circ}$.

Preuve. D'abord on vérifie que le foncteur $\boldsymbol{D}$ conserve les équivalences d'homotopie. Puisque

$$
G r_{j}^{F}\left(\operatorname{Ham}_{\Theta}^{F}\left((L, F),\left(K_{X}^{i}, F\right)\right) \simeq \operatorname{Ham}_{\Theta}\left(G r_{-j}^{F} L, K_{X}^{i}\right),\right.
$$

on voit que

$$
\begin{aligned}
\operatorname{Gr}_{j}^{G}\left(\boldsymbol{D}\left(M^{*}, F\right)\right) & \simeq \boldsymbol{D}\left(G r_{-j}^{G}\left(M^{*}, F\right)\right) \\
& \simeq \boldsymbol{D}\left(G r_{-j}^{F} G r_{-j}^{G} M^{*} \otimes_{\mathcal{O}}\left(\mathscr{D}_{X}, F[-j]\right)\right),
\end{aligned}
$$

donc $\boldsymbol{D}\left(M^{\circ}, F\right)$ est acyclique filtré, si $\left(M^{\circ}, F\right)$ l'est (d'après 2.1.11). Pour la dernière assertion, il suffit de vérifier que $\operatorname{Ham}_{\mathscr{D}}^{F}\left(\left(M^{\circ}, F\right), K^{\circ} \otimes\left(\mathscr{D}_{X}, F\right)\right)$ est acyclique filtré pour $\left(M^{*}, F\right) \in K^{-} F_{i}^{f}\left(\mathscr{D}_{X}\right)$ et $K^{\circ} \in K^{+}\left(\mathscr{D}_{X}\right)$, si $K^{\circ}$ est acyclique et si les $K^{j}$ sont injectifs sur $\mathcal{O}_{X}$. Mais cela se voit par le même argument que ci-dessus (i.e. par le passage à $G r_{j}^{G}$ ); d'où l'assertion.

2.4.5. Lemme. Le foncteur $\boldsymbol{D}$ induit les foncteurs contravariants:

$$
\begin{aligned}
& D: D_{\operatorname{coh}}^{b} F_{i}^{f}\left(\mathscr{D}_{X}\right) \rightarrow D_{\mathrm{coh}}^{b} F_{i}^{f}\left(\mathscr{D}_{X}\right) \\
& \boldsymbol{D}: D_{\mathrm{coh}}^{b} F\left(\mathscr{D}_{X}\right) \rightarrow D_{\mathrm{coh}}^{b} F\left(\mathscr{D}_{X}\right) .
\end{aligned}
$$

Preuve. D'après 2.1.16, il suffit de vérifier la première. Par définition on a $\boldsymbol{D}\left(D_{\mathrm{coh}}^{b} F_{i}^{f}\left(\mathscr{D}_{X}\right)\right) \subset D_{\mathrm{coh}}^{+} F_{i}^{f}\left(\mathscr{D}_{X}\right)^{b}$ (cf. 2.1 pour les notations), car $\mathcal{E x t}_{\mathcal{O}}^{i}\left(M, \omega_{X}\left[d_{X}\right]\right)$ $=0$ pour $i>0$, si $M$ est un $\mathcal{O}_{X}$-Module cohérent; alors l'assertion résulte de 2.1.14, car la condition (2.1.15.1) est cohomologique.

2.4.6. Remarques. (1) On peut définir

$$
D: D_{(\mathrm{coh})} F_{i}^{f}\left(\mathscr{D}_{X}\right) \rightarrow D_{(\mathrm{coh})} F_{i}^{f}\left(\mathscr{D}_{X}\right)
$$


si on utilise le produit direct pour la définition de complexe simple associé. On peut aussi construire l'isomorphisme canonique:

$$
\boldsymbol{D} \circ \boldsymbol{D} \underset{\rightarrow}{\rightarrow} \text { id } \text { sur } D_{\text {coh }} F_{i}^{f}\left(\mathscr{D}_{X}\right) .
$$

(2) D'après V.D. Golovin (Soviet Math. Dokl. Vol. 16 (1975) p. 854), dim. injo $\omega_{X} \leq d_{X}$. Ce qui nous permet de prendre un complexe borné pour $K_{X}^{\circ}$ dans 2.4.3; alors le lemme 2.4.5 et le remarque ci-dessus sont trivials.

2.4.7. Soient $(L, F) \in M F^{f}\left(\Theta_{X}\right.$, Diff $)$ et $\left(L^{\prime}, F\right) \in M F\left(\mathcal{O}_{X}\right.$, Diff $)$, on définit le faisceau filtré $\operatorname{Hom}_{\mathrm{Diff}}^{F}\left((L, F),\left(L^{\prime}, F\right)\right)$ dont la filtration $F$ est exhaustive, par

$$
F_{p}:=\operatorname{Ham}_{\text {Diff }}\left((L, F),\left(L^{\prime}, F[-p]\right)\right),
$$

où les inclusions $F_{p} \rightarrow F_{p+1}$ sont définies par la composition avec les morphismes diffèrentiels filtrés:

$$
\text { id: }\left(L^{\prime}, F[-p] \rightarrow\left(L^{\prime}, F[-p-1]\right),\right.
$$

cf. 2.2.4. D'après 2.2 .5 , on a l'isomorphisme canonique:

$$
D R: \operatorname{Ham}_{\mathcal{D}}^{F}\left((M, F),\left(M^{\prime}, F\right)\right) \stackrel{\sim}{\rightarrow} \operatorname{Ham}_{\mathrm{Diff}}^{F}\left((L, F),\left(L^{\prime}, F\right)\right)
$$

où $(M, F)=D R^{-1}(L, F)$, etc. Puisque $(M, F)$ et $\left(M^{\prime}, F\right)$ sont munis d'une structure de $\mathcal{O}_{X}$-Module associée au foncteur $\otimes_{\mathcal{O}}$ comme dans 2.4 .1 , les $\boldsymbol{C}$ Modules filtrés de (2.4.7.1) sont munis d'une structure de $\mathcal{O}_{X}$-bi-Module. (Notons que cette structure sur le deuxième est définie par la composition avec l'action de $\mathcal{O}_{X}$.) Désormais on n'utilisera que cette structure de $\mathcal{O}_{X^{-}}$ Module associée à celle de $\left(M^{\prime}, F\right)$, car c'est reliée à la structure de $\mathscr{D}_{X}$-Module sur $(\boldsymbol{D}(M, F))^{i}$ dans le cas $\left(L^{\prime}, F\right)=\left(K_{X}^{i}, F\right)$. Par cette structure, on a:

$$
\operatorname{Ham}_{\mathrm{Diff}}^{F}\left((L, F),\left(L^{\prime}, F\right)\right) \in M F\left(\Theta_{X}, \mathrm{Diff}\right) .
$$

2.4.8. Lemme. Soient $\left(L^{*}, F\right) \in C F^{f}\left(\mathcal{O}_{X}\right.$, Diff $)$ et $\left(L^{\prime \cdot}, F\right) \in C F\left(\mathcal{O}_{X}\right.$, Diff $)$, alors $F$ Hom $_{\text {Diff }}\left(\left(L^{*}, F\right),\left(L^{\prime *}, F\right)\right)$ est un complexe double de $M F\left(\Theta_{X}\right.$, Diff $)$ par la structure de $\mathcal{O}_{X}$-Module définie ci-dessus.

Preuve. La différentielle de $\left(L^{\bullet}, F\right)$ induit des morphismes différentiels filtrés (de degré 0 ) par (2.4.7.1), donc l'assertion résulte du lemme suivant:

2.4.9. Lemme. Soient $\left(L^{\prime \bullet}, F\right) \in C F\left(\mathcal{O}_{X}\right.$, Diff $)$ et $\left(M^{\prime \prime}, F\right)=D R^{-1}\left(L^{\prime \prime}, F\right)$. Alors $\left(M^{\prime}, F\right)$ est un complexe de $M F\left(\mathcal{O}_{X}\right.$, Diff $)$ par la structure de $\mathcal{O}_{X}$-Module associée à $\otimes_{\mathcal{O}} . \quad \operatorname{Pour}(L, F) \in M F^{f}\left(\mathcal{O}_{X}\right.$, Diff $)$, on a

$$
H o_{\text {Diff }}^{F} m\left((L, F),\left(L^{\prime \cdot}, F\right)\right) \in C F\left(\mathcal{O}_{X}, \text { Diff }\right)
$$


par la structure de $\mathcal{O}_{X}$-Module definie dans 2.4.7.

Preuve. On a des isomorphismes canoniques comme $\mathscr{D}_{X}$-bi-Modules filtrés:

$$
\left(\left(L^{\prime i}, F\right) \otimes_{\mathcal{O}}\left(\mathscr{D}_{X}, F\right)\right) \otimes_{\mathcal{O}}\left(\mathscr{D}_{X}, F\right) \underset{\rightarrow}{\rightarrow}\left(L^{\prime i}, F\right) \otimes_{\mathcal{O}}\left(\left(\mathscr{D}_{X}, F\right) \otimes_{\mathcal{O}}\left(\mathscr{D}_{X}, F\right)\right)
$$

définis par $(u \otimes P) \otimes Q \mapsto u \otimes(P \otimes Q)$, où on utilise la structure de $\mathscr{D}_{X}$-Module à gauche sur $\mathscr{D}_{X}$ pour définir $\mathscr{D}_{X} \otimes_{\mathcal{O}} \mathscr{D}_{X}$. Puisque le dernier terme est isomorphe à

$$
\left(\left(L^{\prime i}, F\right) \otimes_{\mathcal{O}}\left(\mathscr{D}_{X}, F\right)\right) \otimes_{\mathscr{D}}\left(\left(\mathscr{D}_{X}, F\right) \otimes_{\mathcal{O}}\left(\mathscr{D}_{X}, F\right)\right),
$$

on obtient un complexe de $M F_{i}\left(\mathscr{D}_{X}\right)$, dont l'image par le foncteur $D R$ est isomorphe au complexe $D R^{-1}\left(L^{\prime}, F\right)$ par l'isomorphisme canonique défini cidessus; d'où la première assertion. Soit $(M, F)=D R^{-1}(L, F)$, alors

$$
\operatorname{Ham}_{\mathscr{D}}^{F}\left((M, F),\left(M^{\prime i}, F\right)\right) \otimes_{\mathcal{O}}\left(\mathscr{D}_{X}, F\right) \simeq \operatorname{Ham}_{\mathscr{D}}^{F}\left((M, F),\left(M^{\prime i}, F\right) \otimes_{\mathcal{O}}\left(\mathscr{D}_{X}, F\right)\right)
$$

comme $\mathscr{D}_{X}$-Modules filtrés, donc on obtient un complexe de $M F_{i}\left(\mathscr{D}_{X}\right)$, dont l'image par $D R$ est $\operatorname{Ham}_{\mathrm{Diff}}^{F}\left((L, F),\left(L^{\prime \bullet}, F\right)\right)$; d'où la dernière assertion. Ce qui achève la preuve de 2.4.8.

2.4.10. Lemme. Soient $(L, F)$ et $\left(L^{\prime}, F\right)$ comme dans 2.4 .7 , alors le quasiisomorphisme filtré dans $C F\left(\mathcal{O}_{X}\right.$, Diff $)$ :

$$
\widetilde{D R}\left(D R^{-1}\left(L^{\prime}, F\right)\right) \rightarrow\left(L^{\prime}, F\right), c f .(2.2 .10 .7)
$$

induit le quasi-isomorphisme filtré:

(2.4.10.1) $\operatorname{Ham}_{\mathrm{Diff}}^{F}\left((L, F), D R\left(\widetilde{D R}^{-1}\left(L^{\prime}, F\right)\right) \rightarrow \operatorname{Ham}_{\mathrm{Diff}}^{F}\left((L, F),\left(L^{\prime}, F\right)\right)\right.$.

Preuve. Soit $(M, F)=D R^{-1}(L, F)$, etc., alors le premier terme de (2.4.10.1) est isomorphe à :

$$
\begin{aligned}
& \operatorname{Ham}_{\mathscr{D}}^{F}\left((M, F), D R^{-1}\left(\widetilde{D R}\left(M^{\prime}, F\right)\right)\right) \\
& \quad \simeq \widetilde{D R}\left(\operatorname{Ham}_{\mathscr{D}}^{F}\left((M, F),\left(M^{\prime}, F\right)\right) \otimes_{\mathcal{O}}\left(\mathscr{D}_{X}, F\right)\right)
\end{aligned}
$$

car $D R^{-1}(\widetilde{D R}(M, F)) \simeq \widetilde{D R}\left(D R^{-1}(M, F)\right)$, où on utilise 2.4.2. On vérifie que le morphisme (2.4.10.1) coïncide avec (2.2.10.7) appliqué à $F$ Hom $\mathscr{D}((M, F)$, $\left.\left(M^{\prime}, F\right)\right)$; d'où l'assertion.

2.4.11. Soit $\left(L^{\bullet}, F\right) \in C^{-} F^{f}\left(\Theta_{X}\right.$, Diff $)$. Soit $\left(K_{X}^{\bullet}, F\right)$ comme dans 2.4.3. Alors par l'isomorphisme (2.4.3.1),

$$
\boldsymbol{D}\left(L^{\bullet}, F\right):=\operatorname{Ham}_{\mathcal{O}}^{F}\left(\left(L^{\bullet}, F\right),\left(K_{X}^{\cdot}, F\right)\right)
$$


est un complexe de $M F^{f}\left(\mathcal{O}_{X}\right.$, Diff $)$ tel que $D R^{-1} \boldsymbol{D}\left(L^{*}, F\right) \simeq \boldsymbol{D}\left(D R^{-1}\left(L^{*}, F\right)\right)$. D'après 2.4 .4 et 2.4 .5 , on obtient le foncteur contravariant

$$
\text { D: } D_{\mathrm{coh}}^{b} F^{f}\left(\mathcal{O}_{X}, \text { Diff }\right) \rightarrow D_{\text {coh }}^{b} F^{f}\left(\mathcal{O}_{X}, \text { Diff }\right)
$$

compatible avec $D R^{-1}$. Puisque

$$
\boldsymbol{D}\left(D R^{-1}\left(L^{*}, F\right)\right) \simeq \operatorname{Ham}_{\mathrm{Diff}}^{F}\left(\left(L^{*}, F\right),\left(K_{X}^{*}, F\right)\right)
$$

si on utilise $r$ pour $\operatorname{Ham}_{\mathscr{D}}$, on obtient l'isomorphisme canonique dans $D^{+} F\left(\mathcal{O}_{X}\right.$, Diff $)$ :

$$
\text { (2.4.11.2) } \quad \boldsymbol{D}\left(L^{\cdot}, F\right) \approx \operatorname{Ham}_{\text {Diff }}^{F}\left(\left(L^{\cdot}, F\right),\left(\tilde{K}_{X}^{\cdot}, F\right)\right)
$$

d'après (2.2.10.7), où $\left(\tilde{K}_{X}^{\cdot}, F\right):=\tilde{D R}\left(K_{X}^{\cdot}, F\right) \in C^{+} F^{f}\left(\mathcal{O}_{X}\right.$, Diff). (Notons que $D R^{-1}\left(\tilde{K}_{X}^{\cdot}, F\right) \simeq \widetilde{D R}\left(\left(K_{X}^{\cdot}, F\right) \otimes\left(\mathscr{D}_{X}, F\right)\right)$.)

Soit Forget: $D F\left(\mathcal{O}_{X}\right.$, Diff $) \rightarrow D\left(C_{X}\right)$ le foncteur naturel, alors Forget $\left(\tilde{K}_{X}^{*}, F\right) \simeq \boldsymbol{C}_{X}\left(d_{X}\right)\left[2 d_{X}\right] \simeq a_{X}^{!} \boldsymbol{C}$. On définit le morphisme fonctoriel

(2.4.11.3) Forget $\left(\boldsymbol{D}\left(L^{\bullet}, F\right)\right) \rightarrow \boldsymbol{D}\left(\right.$ Forget $\left.\left(L^{\bullet}, F\right)\right)$

par le morphisme canonique:

Forget $\left(\operatorname{Ham}_{\mathrm{Diff}}^{F}\left(\left(L^{\cdot}, F\right),\left(\tilde{K}_{\dot{X}}^{\cdot}, F\right)\right) \rightarrow \operatorname{Ham}_{C}\left(\operatorname{Forget}\left(L^{\cdot}, F\right), a_{X}^{!} C\right)\right.$.

Soit $D_{\mathrm{hol}}^{b} F^{f}\left(\mathcal{O}_{X}\right.$, Diff) (resp. $\left.D_{\mathrm{hol}}^{b} F\left(\mathscr{D}_{X}\right)\right)$ la sous-catégorie pleine de $D_{\mathrm{coh}}^{b} F^{f}\left(\mathcal{O}_{X}\right.$, Diff) (resp. $D_{\text {coh }}^{b} F\left(\mathscr{D}_{X}\right)$ ) des objets $\left(L^{*}, F\right)$ (resp. $\left(M^{*}, F\right)$ ) tels que les $\mathscr{H}^{i}\left(D R^{-1}\left(L^{\bullet}\right)\right)\left(\right.$ resp. $\left.\mathscr{H}^{i}\left(M^{*}\right)\right)$ soient des $\mathscr{D}_{X}$-Modules holonomes pour tout $i$, alors cette sous-catégorie est stable par $\boldsymbol{D}$.

2.4.12. Proposition. Avec les notations ci-dessus, le morphisme (2.4.11.3) est un isomorphisme pour $\left(L^{*}, F\right) \in D_{\mathrm{hol}}^{b} F^{f}\left(\mathcal{O}_{X}\right.$, Diff $)$, i.e. Forget $\circ D \simeq D \circ$ Forget sur $D_{\mathrm{hol}}^{b} F^{f}\left(\Theta_{X}\right.$, Diff) et (Forget $\left.\circ D R\right) \circ D \simeq D \circ($ Forget $\circ D R)$ sur $D_{\mathrm{hol}}^{b} F\left(\mathscr{D}_{X}\right)$.

Preuve. Soit $\tau_{\leq p}(p \in Z)$ la filtration canonique [6, 1 II (1.4.6)] sur des complexes de $\mathscr{D}_{X}$-Modules filtrés, telle que $\tau_{S p} \oplus=\oplus \tau_{S p}$, cf. 2.11. On a alors une filtration sur $\widetilde{D R}\left(D R^{-1}\left(L^{*}, F\right)\right)$ par:

$$
\widetilde{D R}\left(\tau_{\leq p} D R^{-1^{*}}(L, F)\right) \quad(p \in \boldsymbol{Z}),
$$

donc on peut supposer que $\mathscr{H}^{i}\left(\oplus D R^{-1}\left(L^{*} F\right)\right)=0$ pour $i \neq 0$. Puisque l'assertion est locale, on peut remplacer $\left(L^{*}, F\right)$ par un complexe borné de $\mathscr{D}_{X}$-Modules filtrés libres de type fini comme dans 2.1.17, par le même argument que dans la preuve le 2.1.16. On a alors 


$$
\boldsymbol{D}\left(L^{\cdot}, F\right) \simeq \operatorname{Ham}_{\mathcal{O}}^{F}\left(\left(L^{\bullet}, F\right),\left(\omega_{X}\left[d_{X}\right], F\right)\right)
$$

où la différentielle du deuxième complexe est définie comme dans 2.4.11. D'après Kashiwara [16], on a

$$
\text { Forget }\left(L^{\bullet}, F\right) \text {, Forget }\left(\boldsymbol{D}\left(L^{\bullet}, F\right)\right) \in D_{c}^{b}\left(\boldsymbol{C}_{X}\right) \text {. }
$$

Par le même argument que dans 2.4.11, on a

$$
\begin{aligned}
\boldsymbol{D}\left(L^{*}, F\right) & \simeq \operatorname{Hom}_{\mathrm{Diff}}^{F}\left(\left(L^{\cdot}, F\right),\left(\Omega_{X}^{\cdot}\left(d_{X}\right)\left[2 d_{X}\right], F\right)\right) \\
& \simeq D R\left(\widetilde{D R^{-1}}\left(\operatorname{Hom}_{\mathcal{O}}^{F}\left(\left(L^{\cdot}, F\right),\left(\omega_{X}\left[d_{X}\right], F\right)\right)\right)\right) .
\end{aligned}
$$

Soient $x \in X$ et $i_{x}:\{x\} \hookrightarrow X$ l'inclusion naturelle. On définit le foncteur $i_{x}^{!}$par

$$
i_{x}^{!} A^{\bullet}=\operatorname{Hom}\left(\left(i_{x}\right)_{*} \boldsymbol{Z}, \mathcal{g}\left(A^{\bullet}\right)\right)
$$

pour un complexe de faisceaux $A^{\circ}$, où $\mathscr{G}\left(A^{\circ}\right)$ est le complexe simple associé au complexe double de la résolution canonique flasque tronquée de $A^{\circ}$. On a alors un diagramme commutatif:

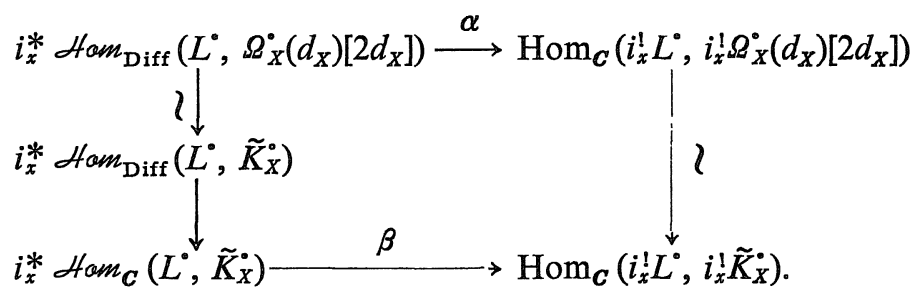

Puisque Forget $\left(\boldsymbol{D}\left(L^{\circ}, F\right)\right) \simeq$ Ham $_{\text {Diff }}\left(L^{\cdot}, \Omega_{X}^{\circ}\left(d_{X}\right)\left[2 d_{X}\right]\right)$ (car $L^{j}$ sont cohérents), il suffit de vérifier que $\alpha$ est bijectif, car $\beta$ est clairement bijectif, cf. par exemple [32]. Soit $\sigma$ la filtration bête de $L^{*}$ (cf. [6, II (1.4.7)]), alors elle induit une cofiltration (dont la filtration correspondante sera notée $G$ ) sur la source et le but de $\alpha$, compatible avec $\alpha$. D'après (2.4.12.1-2), $G r_{i}^{G} \alpha$ s'écrit:

$$
G r_{i}^{G} \alpha: i_{x}^{*} \operatorname{Ham}_{\mathcal{O}}\left(L^{-i}, \omega_{X}\right)\left[d_{X}\right] \rightarrow \operatorname{Hom}_{C}\left(H_{\{x\}}^{d_{X}} L^{-i}, \boldsymbol{C}\right)\left[d_{X}\right] .
$$

Puisque le but de $G r_{i}^{G} \alpha$ est isomorphe à

$$
\operatorname{Hom}_{C}\left(H_{\{x\}}^{d_{X}} L^{-i}, H_{\{x\}}^{d} \Omega_{X}^{\circ}\left(d_{X}\right)\right)\left[2 d_{X}\right],
$$

l'accouplement

$$
\alpha_{i}^{\prime}: i_{x}^{*} \operatorname{Hamo}_{\mathcal{O}}\left(L^{-i}, \omega_{X}\right) \times H_{\{x\}}^{d_{X}^{X}} L^{-i} \rightarrow C
$$

correspondant à $G r_{i}^{G} \alpha$, est défini par le morphisme trace (ou le morphisme de résidu): 


$$
\operatorname{Tr}: H_{[x]}^{d X} \omega_{X} \rightarrow \boldsymbol{R} \Gamma_{\{x\}} \Omega_{X}^{\cdot}\left(d_{X}\right)\left[2 d_{X}\right] \cong \boldsymbol{C} .
$$

(Notons que $\omega_{X}=\Omega_{X}^{d} X\left(d_{X}\right)$ par définition, cf. (2.0.2).) Puisque les suites spectrales de $G$ dégénèrent en $E_{2}$, il suffit de vérifier que les $H^{i} G r^{G} \alpha$ sont bijectifs. Par la dualité de Serre, les $\alpha_{i}^{\prime}$ sont des accouplements topologiquement parfaits. Par les deux lemmes suivants, les différentielles des complexes $i_{*}^{x} \not m m_{O}\left(L^{*}, \omega_{X}\right)$ et $H_{\{x\}}^{d_{X} X} L^{\cdot}$ sont duales l'une de l'autre par cette dualité. Puisque $\boldsymbol{D} L^{*} \in D_{c}^{b}\left(\boldsymbol{C}_{X}\right)$, on obtient la dualité parfaite sur leurs cohomologies; d'où l'assertion (comparer à [16]).

2.4.13. Lemme. Soit $P \in \operatorname{Ham}_{\mathrm{Diff}}\left(\mathcal{O}_{X}, \mathcal{O}_{X}\right) \simeq \mathscr{D}_{X}$. Soit $P^{*}: \operatorname{Ham}_{\mathcal{O}}\left(\mathcal{O}_{X}, \omega_{X}\right)$ $\rightarrow \operatorname{Ham}_{\mathcal{O}}\left(\mathcal{O}_{X}, \omega_{X}\right)$ la transposé de $P$ définie comme dans 2.4.11. Alors par l'isomorphisme canonique:

$$
\operatorname{Ham} O\left(O_{X}, \omega_{X}\right) \simeq \omega_{X}
$$

$P^{*}$ s'identifie à l'action de $P$ à droite sur $\omega_{X}$.

2.4.14. Lemme. Soit $\langle\rangle:, \omega_{X, x} \times H_{\{x\}}^{d_{X}^{X}} \mathcal{O}_{X} \rightarrow C$ l'accouplement comme cidessus, on a alors

$$
\langle u P, v\rangle=\langle u, P v\rangle
$$

pour $u \in \omega_{X, x}, v \in H_{[x]}^{d_{X}} \mathcal{O}_{X}$ et $P \in \mathscr{D}_{X, x}$

(Les démonstrations de ces deux lemmes sont laissées au lecteurs.)

2.4.15. Remarques.

(1) La définition du foncteur dual sur $D_{\text {coh }}^{b} F\left(\mathscr{D}_{X}\right)$ est compatible avec la définition usuelle, par le foncteur naturel: $D_{\mathrm{coh}}^{b} F\left(\mathscr{D}_{X}\right) \rightarrow D_{\mathrm{coh}}^{b}\left(\mathscr{D}_{X}\right)$. De même le foncteur Forgeto $D R: D F\left(\mathscr{D}_{X}\right) \rightarrow D\left(\boldsymbol{C}_{X}\right)$ est compatible avec la définition usuelle de $D R: D\left(\mathscr{D}_{X}\right) \rightarrow D\left(\boldsymbol{C}_{X}\right)$, par le foncteur naturel ci-dessus. Ici, le foncteur entre les catégories dérivées induit par $\widetilde{D R}$ est noté aussi $D R$.

(2) Soit $M_{\text {hol }}\left(\mathscr{D}_{X}\right)$ la catégorie des $\mathscr{D}_{X}$-Modules holonomes, cf. [16]. Alors cette catégorie est stable par le foncteur dual (par sa compatibilité avec la microlocalisation). D'après Kashiwara [loc. cit], le foncteur $D R$ induit le foncteur exact:

$$
D R: M_{\mathrm{hol}}\left(\mathscr{D}_{X}\right) \rightarrow \operatorname{Perv}\left(\boldsymbol{C}_{X}\right),
$$

(voir [1] pour la définition de $\operatorname{Perv}\left(\boldsymbol{C}_{X}\right)$ ) car il a démontré la demi-perversité et la dualité parfaite:

$$
i_{x}^{*} D R(\boldsymbol{D} M) \times i_{x}^{!} D R(M) \rightarrow \boldsymbol{C}
$$


i.e. $i_{x}^{*} D R(D M) \simeq i_{x}^{*} \boldsymbol{D}(D R(M))$, pour tout $i_{x}:\{x\} \hookrightarrow X$. Notons que le foncteur (2.4.15.1) est exacte et fidèle, car $D R(\operatorname{Im} u)=\operatorname{Im}(D R(u))$ pour un morphisme $u$ de $M_{\text {hol }}\left(\mathscr{D}_{X}\right)$, et $M=0$ si $D R(M)=0$ pour $M \in M_{\mathrm{ho}}\left(\mathscr{D}_{X}\right)$.

(3) Posons Sol $(M)=\boldsymbol{R} \operatorname{Ham}_{\mathcal{D}}\left(M, \omega_{X}\right)\left[d_{X}\right]$ pour $M \in M_{\text {hol }}\left(\mathscr{D}_{X}\right)$, alors on peut vérifier que

$$
\operatorname{Sol}(M) \simeq D R(D M) \text {. }
$$

Donc on obtient le foncteur Sol: $M_{\text {hol }}\left(\mathscr{D}_{X}\right) \rightarrow \operatorname{Perv}\left(C_{X}\right)$, et la dualité (2.4.15.2) s'exprime en

$$
i_{x}^{*} \operatorname{Sol}(M) \simeq i_{x}^{*} \boldsymbol{D}(D R(M)) .
$$

D'après [18] (voir aussi [23]) ou peut construire un isomorphisme global

$$
\text { Sol }(M) \simeq D(D R(M)) \text {. }
$$

(Comparer à 2.4.12-14; dans [23] on remplace la dualité de Serre pour la cohomologie locale par cette dualité pour la cohomologie à supports compacts.)

2.4.16. Remarque. Avec les notations et les hypothèses de (2.4.3.1), soit $\left(x_{1}, \cdots, x_{d_{X}}\right)$ un système de coordonnées locales, alors $\phi \in(\boldsymbol{D}(M, F))^{i}$ s'écrit:

$$
\sum_{\nu} \phi_{\nu} \otimes \partial^{y} \quad \text { avec } \phi_{y} \in \operatorname{Ham}_{\mathcal{O}}\left(L, K_{\bar{X}}^{-i}\right)
$$

par l'isomorphisme (2.4.3.1); ce qui correspond à

$$
\left[u \mapsto \Sigma_{v} \phi_{y}(u) \partial^{\nu}\right] \in \operatorname{Ham}_{\mathrm{Diff}}\left(L, K_{\bar{X}}^{-i}\right),
$$

par l'isomorphisme (2.4.11.1).

\subsection{Dualité pour un morphisme propre}

2.5.1. Soit $f: X \rightarrow Y$ un morphisme propre de variétés complexes lisses. Soient $\left(K_{X}^{\circ}, F\right)$ et $\left(\tilde{K}_{X}^{\cdot}, F\right)$ comme dans 2.4 .3 a et 2.4 .11 (de même pour $Y$ ). Soit $\tilde{I}_{X}^{\circ}$ le complexe double des courants au sens de Schwartz, i.e. $\Gamma\left(U, \widetilde{I}_{X}^{\circ}\right)$ s ont des sous-complexes doubles de $\operatorname{Ham}_{C}\left(\Gamma_{c}\left(U,{ }^{\infty} \Omega_{X}^{* *}\right), C\right)$ pour tout ouvert $U$, où ${ }^{\infty} \Omega_{X}^{*}$ est le complexe double des formes $C^{\infty}$. Soit $\tilde{I}_{X}^{\circ}$ le complexe simple associé à $\tilde{I}_{X}^{\bullet}$ soit $I_{X}^{*}$ le complexe quotient de $\tilde{I}_{X}^{\cdot}$ tel que $I_{X}^{p}=\tilde{I}_{X}^{o p}$, alors $\omega_{X}\left[d_{X}\right] \sim_{\rightarrow}^{\rightarrow} I_{X}^{*}$ et $\widetilde{D R}\left(I_{X}^{*}\right) \underset{\rightarrow}{\rightarrow} \widetilde{I}_{X}^{*}$. On définit la filtration $F$ sur $I_{X}^{\cdot}$ et $I_{X}^{* \bullet}$ comme sur $K_{X}^{*}$ et $\widetilde{K}_{X}^{\cdot}$, cf. 2.4.3. et 2.4.11. (De même pour $I_{Y}^{\circ}$ et $\tilde{I}_{Y}^{\circ}$ )

Par définition de $K_{X}^{\circ}$, on peut supposer qu'il existe un quasi-isomorphisme $I_{X}^{*} \underset{\rightarrow}{\rightarrow} K_{X}^{\cdot}$, ce qui induit les quasi-isomorphismes filtrés:

$$
\left(I_{X}^{*}, F\right) \underset{\rightarrow}{\rightarrow}\left(K_{X}^{*}, F\right), \quad\left(\tilde{I}_{X}^{\cdot}, F\right) \stackrel{\sim}{\rightarrow}\left(\tilde{K}_{X}^{*}, F\right) .
$$


(De même pour $Y$.)

Par le "push-down" des courants, on a le morphisme trace:

$$
\operatorname{Tr}_{f}: f_{*}\left(\tilde{I}_{X}^{*}, F\right) \rightarrow\left(\tilde{I}_{Y}^{\cdot}, F\right)
$$

dans $K_{\text {coh }}^{b} F^{f}\left(\mathcal{O}_{Y}\right.$, Diff $)$. Donc on obtient le morphisme dans $K^{+} F\left(\mathscr{D}_{Y}\right)$ :

$$
D R^{-1} f_{*}\left(\tilde{I}_{X}^{\cdot}, F\right) \stackrel{D R^{-1}(T r)}{\longrightarrow} D R^{-1}\left(\tilde{I}_{Y}^{\cdot}, F\right) \rightarrow\left(I_{Y}^{\cdot}, F\right) \rightarrow\left(K_{Y}^{*}, F\right)
$$

d'après (2.2.10.6). Ici, on peut supposer que $K_{Y}^{i}$ sont des $\mathscr{D}_{Y}$-Modules injectifs. Puisque

$$
D R^{-1} f_{*}\left(\tilde{I}_{X}^{\cdot}, F\right) \rightarrow D R^{-1} f_{*}\left(\tilde{K}_{X}^{\cdot}, F\right)
$$

est un quasi-isomorphisme filtré, le morphisme (2.5.1.2) se factorise par (2.5.1.3) dans $K^{+} F\left(\mathscr{D}_{Y}\right)$. (En effet, l'assertion est claire si on oublie la filtration; mais le morphisme et l'homotopie respectent la filtration car $F_{-1}=0$ et $F_{0} K_{Y}^{*}=K_{Y}^{*}$.) Donc on obtient le diagramme commutatif dans $K^{+} F\left(\mathcal{O}_{Y}\right.$, Diff $)$ :

$$
\begin{array}{cc}
\widetilde{D R} \circ D R^{-1} \circ f_{*}\left(\tilde{I}_{X}^{\cdot}, F\right) & \rightarrow \widetilde{D R} \circ D R^{-1} \circ f_{*}\left(\tilde{K}_{X}^{\cdot}, F\right) \\
\downarrow & \downarrow T r \\
\widetilde{D R} \circ D R^{-1}\left(\tilde{I}_{\dot{Y}}, F\right) & \downarrow T r
\end{array}
$$

où les morphismes horizontaux sont des quasi-isomorphismes filtrés. (Cette construction est suggérée par Kashiwara.)

2.5.2. Soit $\left(L^{\cdot}, F\right) \in K_{\text {coh }}^{b} F^{f}\left(\Theta_{X}\right.$, Diff $)$ tel que $G r_{i}^{F} L^{j}$ sont $f_{*}$-acycliques. D'après (2.4.11.2), on a les quasi-isomorphismes filtrés:

$$
\begin{aligned}
& f_{*} \boldsymbol{D}\left(L^{*}, F\right) \leftarrow f_{*} \operatorname{Ham}_{\text {Diff }}^{F}\left(\left(L^{*}, F\right),\left(\tilde{K}_{X}^{\cdot}, F\right)\right) \\
& \boldsymbol{D} f_{*}\left(L^{\cdot}, F\right) \leftarrow \operatorname{Ham}_{\text {Diff }}^{F}\left(f_{*}\left(L^{\cdot}, F\right),\left(\tilde{K}_{Y}^{\cdot}, F\right)\right) .
\end{aligned}
$$

car $G r_{p}^{F} \operatorname{Ham}_{\Theta}^{F}\left(\left(L^{i}, F\right),\left(\tilde{K}_{X}^{i}, F\right)\right) \simeq \bigoplus_{q} \operatorname{Ham} \Theta\left(G r_{q}^{F} L^{i}, G r_{p+q}^{F} \tilde{K}_{X}^{j}\right)$ sont flasques. Par définition, l'action de $\mathcal{O}_{X}$ (ou de $f^{-1} \mathcal{O}_{Y}$ ) sur chaque Hom $_{\text {Diff }}^{f}\left(L^{i}, \widetilde{K}_{X}^{j}\right)$ $\left(:=\bigcup_{p} \operatorname{Ham}_{\mathcal{O}}\left(L^{i}, K_{X}^{j} \otimes F_{p} \mathscr{D}_{X}\right)\right)$ est définie par la composition avec l'action naturelle de $\mathcal{O}_{X}$ (ou de $f^{-1} \mathcal{O}_{Y}$ ) sur $\tilde{K}_{X}^{j}$, donc on obtient le morphisme dans $K_{\text {coh }}^{+} F\left(\mathcal{O}_{Y}\right.$, Diff $)$ :

$$
f_{*} \operatorname{Ham}_{\text {Diff }}^{F}\left(\left(L^{*}, F\right),\left(\tilde{K}_{X}^{*}, F\right)\right) \rightarrow \operatorname{Ham}_{\text {Diff }}^{F}\left(f_{*}\left(L^{*}, F\right), f_{*}\left(\tilde{K}_{X}^{*}, F\right)\right) .
$$

D'après (2.5.2.1-2), (2.4.10.1) et (2.5.1.4), on a le morphisme fonctoriel dans $D_{\text {coh }}^{+} F\left(\mathcal{O}_{Y}\right.$, Diff) (donc dans $D_{\text {coh }}^{b} F^{f}\left(\mathcal{O}_{Y}\right.$, Diff) d'après $2.2 .10,2.1 .14$ et 2.1.12):

$$
f_{*} \boldsymbol{D}\left(L^{\bullet}, F\right) \rightarrow \boldsymbol{D} f_{*}\left(L^{\cdot}, F\right)
$$


2.5.3. Proposition. Le morphisme (2.5.2.3) induit un isomorphisme fonctoriel

$$
f_{*} \boldsymbol{D} \simeq D f_{*}: D_{\mathrm{coh}}^{b} F^{f}\left(\mathcal{O}_{X}, \text { Diff }\right) \rightarrow D_{\mathrm{coh}}^{b} F^{f}\left(\mathcal{O}_{Y}, \text { Diff }\right)
$$

Preuve. Par dévissage, on peut supposer que $\left(L^{\bullet}, F\right)$ est un complexe de $\mathcal{O}_{X^{-}}$ Modules muni d'une filtration triviale, i.e. $G r_{i}^{F} L^{\bullet}=0$ pour $i \neq 0$. On vérifie que $G r_{i}^{E} f_{*} \boldsymbol{D}\left(L^{\bullet}, F\right)$ et $G r_{i}^{F} \boldsymbol{D} f_{*}\left(L^{\bullet}, F\right)$ sont acycliques pour $i \neq 0$ et que

$$
\begin{aligned}
& G r_{0}^{F} f_{*} D\left(L^{\cdot}, F\right)=f_{*} \mathscr{H}_{\text {yam }}\left(L^{\bullet}, K_{X}^{*}\right) \\
& G r_{0}^{E} D f_{*}\left(L^{\circ}, F\right)=\operatorname{Ham}_{O}\left(f_{*} L^{\cdot}, K_{X}^{*}\right) .
\end{aligned}
$$

De plus, $G r_{0}^{F}$ du quasi-isomorphisme (2.4.10.1) appliqué à $f_{*}\left(L^{\bullet}, F\right)$ et $f_{*}\left(\tilde{K}_{X}^{\bullet}, F\right)$ est l'identité:

$$
\operatorname{Ham}_{O}\left(f_{*} L^{\cdot}, f_{*} K_{X}^{*}\right)=\operatorname{Ham} O\left(f_{*} L^{*}, f_{*} K_{X}^{*}\right)
$$

et $G r_{0}^{F}$ de (2.5.1.4) est un diagramme commutatif dans $K^{+}\left(\mathcal{O}_{Y}\right)$ :

$$
\begin{gathered}
f_{*} I_{X}^{\circ} \rightarrow f_{*} K_{X}^{\circ} \\
\downarrow T r \quad \downarrow T r \\
I_{Y}^{\circ} \rightarrow K_{Y}^{\circ} .
\end{gathered}
$$

Donc $G r_{0}^{F}$ de (2.5.2.3) coïncide avec le morphisme de dualité dans la théorie de dualité analytique [25]; d'où l'assertion.

2.5.4. Corollaire. On a un isomorphisme fonctoriel:

$$
f_{*} \boldsymbol{D} \simeq \boldsymbol{D} f_{*}: D_{\mathrm{coh}}^{b} F\left(\mathscr{D}_{X}\right) \rightarrow D_{\mathrm{coh}}^{b} F\left(\mathscr{D}_{Y}\right) .
$$

Preuve. Cela résulte de (2.2.10.5) et de la compatibilité des foncteurs $f_{*}$ et $\boldsymbol{D}$ avec le foncteur $D R^{-1}$, cf. 2.3.7 et 2.4.11.

2.5.5. Proposition. La dualité (2.5.2.3) sur $D_{\mathrm{hol}}^{b} F\left(\mathcal{O}_{X}\right.$, Diff) est compatible á la dualité topologique par les foncteurs

$$
\text { Forget: } D_{\mathrm{hol}}^{b} F\left(\mathcal{O}_{X} \text {, Diff }\right) \rightarrow D_{c}^{b}\left(\boldsymbol{C}_{X}\right) \quad \text { (de meme pour } Y \text { ) }
$$

et par les isomorphismes fonctoriels:

$$
f_{*} \circ \text { Forget }=\text { Forget } \circ f_{*} \text { et } \quad \boldsymbol{D} \circ \text { Forget }=\text { Forget } \circ \boldsymbol{D}
$$

cf. 2.3.7 et 2.4.12.

Preuve. Par le quasi-isomorphisme canonique:

$$
C_{X}\left(d_{X}\right)\left[2 d_{X}\right] \stackrel{\sim}{\rightarrow} \tilde{I}_{X}^{\cdot} \quad(\text { de même pour } Y \text { ) },
$$


le morphisme $\operatorname{Tr}_{f}: f_{*} \tilde{I}_{X}^{\circ} \rightarrow \tilde{I}_{Y}^{\circ}$ (cf. (2.5.1.1)) représente le morphisme trace dans la théorie de dualité topologique (cf. par exemple [32]). Puisque $\tilde{K}_{Y}^{*}$ est un complexe de $\boldsymbol{C}$-Modules injectifs, le morphisme $\operatorname{Tr}$ dans (2.5.1.4) se factorise par le quasi-isomorphisme: $\widetilde{D R} \circ D R^{-1} \circ f_{*} \widetilde{K}_{X}^{\cdot} \rightarrow f_{*} \widetilde{K}_{X}^{\cdot}$, donc on obtient le dia. gramme commutatif dans $K^{+}\left(\boldsymbol{C}_{Y}\right)$ :

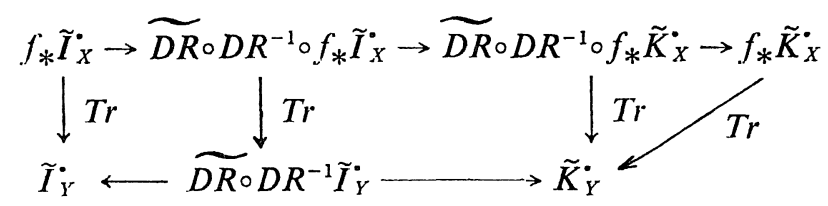

où les morphismes horizontaux sont des quasi-isomorphismes; en particulier, le morphisme $\operatorname{Tr}: f_{*} \tilde{K}_{X}^{*} \rightarrow \tilde{K}_{Y}^{*}$ aussi représente le morphisme trace. Posons

$$
\operatorname{Ham}_{\text {Diff }}^{j}\left(L, \tilde{K}_{X}^{\cdot}\right)=\operatorname{Forget}\left(\operatorname{Ham}_{\text {Diff }}^{F}\left((L, F),\left(\tilde{K}_{X}^{\cdot}, F\right)\right)\right), \text { etc., }
$$

On a alors un diagramme commutatif dans $D^{+}\left(\boldsymbol{C}_{Y}\right)$ :

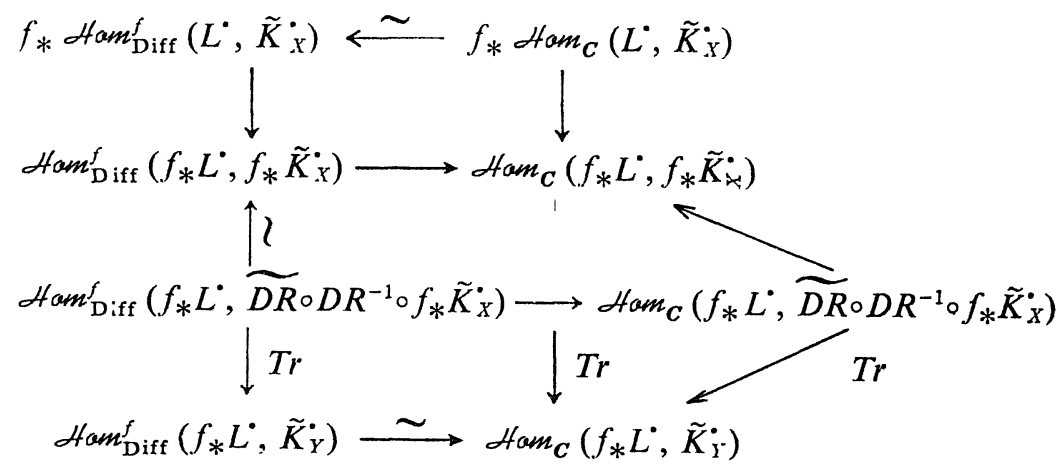

d'où l'assertion.

2.5.6. Corollaire. La dualité de 2.5 .4 sur $D_{\mathrm{hol}}^{b} F\left(\mathscr{D}_{X}\right)$ est compatible avec la dualité topologique, par les foncteurs:

$$
\text { ForgetoDR: } D_{\mathrm{hol}}^{b} F\left(\mathscr{D}_{X}\right) \rightarrow D_{c}^{b}\left(\boldsymbol{C}_{X}\right) \text {, etc. }
$$

Preuve. Cela résulte de 2.5 .5 et de la preuve de 2.5.4.

2.5.7. Remarque. On dit que $\boldsymbol{Z}_{X}\left(d_{X}\right)$ est le faisceau d'orientation de Deligne. On a l'isomorphisme canonique:

$$
a_{X}^{l} \boldsymbol{Z} \simeq \boldsymbol{Z}_{X}\left(d_{X}\right)\left[2 d_{X}\right]
$$

où $a_{X}: X \rightarrow p t$. Le morphisme trace $\operatorname{Tr}: a_{X} \boldsymbol{Z}_{X}\left(d_{X}\right)\left[2 d_{X}\right] \rightarrow \boldsymbol{Z}$ est défini par le diagramme commutatif: 


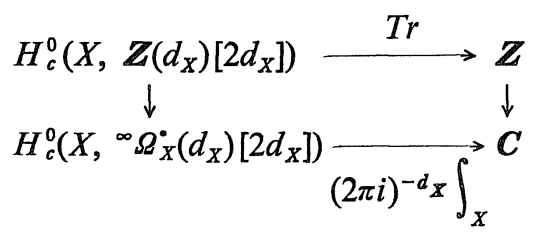

2.5.8. Remarque. Soit $X$ une variété complexe lisse compacte. Soit $K=$ $\left(K_{A},\left(K_{A \otimes Q}, W\right),\left(K_{C}, W, F\right)\right)$ un $A$-complexe de Hodge mixte cohomologique au sens de [6, III (8.1.6)], tel que $K_{A} \in D_{c}^{b}\left(A_{X}\right)$ et que $\left(K_{C}, W, F\right)$ soit représenté par un objet $\left(L^{\bullet}, F\right)$ de $C_{\text {hol }}^{b} F\left(\Theta_{X}\right.$, Diff $)$ muni d'une filtration $W$ dans $C_{\text {hol }}^{b} F\left(\mathcal{O}_{X}\right.$, Diff $)$. Alors on peut définir le dual de $\left(K_{C}, W, F\right)$ par $\mathbb{D}\left(\left(L^{\bullet}, F\right), W\right)$. D'après 2.5.5, on voit que $\left.\boldsymbol{D} K=\left(\boldsymbol{D} K_{A}, \boldsymbol{D}\left(K_{A \otimes Q}, W\right), \boldsymbol{D}\left(\left(L^{\bullet}, F\right), W\right)\right)\right)$ est un $A$ complexe de Hodge mixte cohomologique, et que la dualité naturelle entre $H^{i}\left(X, K_{A}\right)$ et $H^{-i}\left(X, D K_{A}\right)$ est compatible la structure de Hodge mixte. Par exemple, soit $\left(L^{*}, F\right)=\left(\Omega_{X}^{\circ}(\log D), F\right)\left[d_{X}\right]$ avec $G r_{d_{X}+i}^{W}=\left(a_{i}\right)_{*}\left(\Omega_{\tilde{D}^{(i)}}^{\cdot}, F[-i]\right)$ $\left[d_{X}-i\right]$, où $F^{p}=\sigma_{\geq p}$, cf. [loc. cit], et soit $K_{A}=\boldsymbol{R}_{j_{*}} A_{X^{*}}\left[d_{X}\right]$, où $j: X^{*}=X \backslash D \rightarrow X$. (Ici, $X$ est Kähler, et $D$ est un diviseur à croisements normaux.) Alors $D K$ définit une structure de Hodge mixte sur $H_{c}^{\circ}\left(X^{*}, A\right)$, de plus $\mathbb{D}\left(\left(L^{\circ}, F\right), W\right)$ est quasi-isomorphe (bifiltré) au complexe:

$$
\left[\left(a_{0}\right)_{*}\left(\Omega_{\tilde{D}^{(0)}}^{\circ}, F\left[d_{X}\right]\right) \rightarrow \cdots \rightarrow\left(a_{d_{X}}\right)_{*}\left(\Omega_{\tilde{D}^{\left(d_{X}\right)}}^{\circ}, F\left[d_{X}\right]\right)\right]
$$

tel que $G r_{-d_{X^{-i}}}^{W}=\left(a_{i}\right)_{*}\left(\Omega_{\widetilde{D}^{(i)}}^{\bullet}, F\left[d_{X}\right]\right)\left[d_{X}-i\right]$. Notons que $D R^{-1}\left(L^{\bullet}, F\right) \cong\left(\omega_{X}(* D)\right.$, $F$ ), où $F$ est une filtration définie par l'ordre du pole (décalée par $-d_{X}$ ), cf. [loc. cit].

\section{§3. Filtration de Malgrange-Kashiwara et Cycles Évanescents}

Dans cet article, toute filtration rationnelle est indexée discrètement, i.e. il existe un entier $m>0$ tel que $V_{\alpha}=V_{i / m}$ pour tout $\alpha \in \boldsymbol{Q}, i \in \mathbb{Z}$ avec $i / m \leqslant \alpha$ $<i+1 / m$, si $V$ est croissante. On notera $V_{<\alpha}=\bigcup_{\beta<\alpha} V_{\beta}$ et $G r_{\alpha}^{V}=V_{\alpha} / V_{<\alpha}$.

\subsection{Filtration rationnelle De Malgrange-Kashiwara}

Soient $X$ une variété complexe lisse, et $X_{0}$ une sous-variété fermée lisse de codimension 1. On définit $V_{\alpha} \mathscr{D}_{X}=\left\{P \in \mathscr{D}_{X}: P I_{X_{0}}^{j_{0}} \subset I_{X_{0}}^{j-\left[{ }^{\alpha}\right]}\right.$ pour tout $\left.j \in \mathbb{Z}\right\}$ pour $\alpha \in \boldsymbol{Q}$, où $[\alpha]=\max \{i \in \mathbb{Z}: i \leqslant \alpha\}, I_{X_{0}}$ est l'Idéal de $X_{0}$, et $I_{X_{0}}^{j}=\mathcal{O}_{X}$ pour $j \leqslant 0$.

Soient $t$ une équation locale de $X_{0}, \partial_{t}$ un champ de vecteur (localement défini) tel que $\left[\partial_{t}, t\right]=1$.

3.1.1. Définition. Soit $M$ un $\mathscr{D}_{X}$-Module cohérent à droite, une filtration 
rationnelle croissante $V$ est dite la filtration de Malgrange-Kashiwara, si

(3.1.1.1) $\bigcup_{\alpha \in Q} V_{\alpha} M=M$, chaque $V_{\alpha} M$ est un sous $V_{0} \mathscr{D}_{X}$-Module cohérent.

(3.1.1.2) $\left(V_{\alpha} M\right)\left(V_{i} \mathscr{D}_{X}\right) \subset V_{\alpha+i} M$ pour tout $\alpha \in \boldsymbol{Q}, i \in \boldsymbol{Z}$. $\left(V_{\alpha} M\right) t=V_{\alpha-1} M$ si $\alpha<0$.

(3.1.1.3) l'action de $t \partial_{t}-\alpha$ sur $G r_{\alpha}^{V} M$ est nilpotente $(\alpha \in \boldsymbol{Q})$.

Notons que $t \in V_{-1} \mathscr{D}_{X}, \partial_{t} \in V_{1} \mathscr{D}_{X}$, que la condition (3.1.1.3) entraine:

(3.1.1.4) $t: G r_{\alpha}^{V} M \rightarrow G r_{\alpha-1}^{V} M$ et $\partial_{t}: G r_{\alpha-1}^{V} M \rightarrow G r_{\alpha}^{V} M$ sont bijectifs pour $\alpha \neq 0$,

(3.1.1.5) $V_{\alpha+i} M=\left(V_{\alpha} M\right)\left(V_{i} \mathscr{D}_{X}\right)$ pour $\alpha \geqslant 0, i \geqslant 0$,

et que les conditions (3.1.1.2-4) sont indépendantes du choix de $t, \partial_{t}$.

3.1.2. Lemme (Kashiwara [17]). La filtration $V$ est unique si elle existe.

Preuve. Supposons qu'il existe deux filtrations $V$ et $V^{\prime}$. D'après les conditions (3.1.1.1-2) et (3.1.1.5), il existe $a, b \in \boldsymbol{Q}$ tels que

$$
V_{\alpha-a} M \subset V_{\alpha}^{\prime} M \subset V_{\alpha+b} M \text { pour tout } \alpha \in \boldsymbol{Q} ;
$$

en particulier, la filtration induite $\left(G r_{\alpha}^{V} M, V^{\prime}\right)$ est finie. Par la condition (3.1.1.3), on vérifie que $G r_{\alpha}^{V^{\prime}} G r_{\beta}^{V} M=0$ si $\alpha \neq \beta$, donc $V_{a}^{\prime} G r_{\alpha+b}^{V} M=0$ si $b>0$, i.e. $V_{\alpha}^{\prime} \subset$ $V_{a+b-\varepsilon}$ pour $\varepsilon>0$, ce qui entraine l'assertion, car $V$ et $V^{\prime}$ sont discrètement paramétrées.

3.1.3. Lemme. Si Supp $M \subset X_{0}$ et $M$ est cohérent, $M$ admet la filtration rationnelle le long de $X_{0}$, de plus, on a

$$
M=M_{0} \underset{C}{\otimes}\left[\partial_{t}\right], \quad V_{\alpha} M=\bigoplus_{0 \leqslant i \leqslant\left[\alpha^{\alpha}\right]} M_{0} \otimes \partial_{t}^{i}
$$

ou $M_{0}=\operatorname{HamO}_{x}\left(\Theta_{X_{0}}, M\right)(=\operatorname{Ker}(t: M \rightarrow M))$.

Preuve. D'après Kashiwara, on a $M=\bigoplus_{i \geqslant 0} M_{i}$, et $\partial_{t}^{i}: M_{0} \underset{\rightarrow}{\sim} M_{i}, t^{i}: M_{i} \sim M_{0}(i \geqslant$ $0)$, où $M_{i}=\operatorname{Ker}\left(t \partial_{t}-i\right)$; donc on a $M_{0}=\operatorname{HamO}_{x}\left(\Theta_{X_{0}}, M\right), M \simeq M_{0} \underset{\boldsymbol{C}}{\otimes} \boldsymbol{C}\left[\partial_{t}\right]$.

On vérifie facilement que la filtration $V$ définie ci-dessus satisfait les conditions (3.1.1.1-3); d'où l'assertion.

3.1.4. Lemme. L'action de $t$ sur $V_{\alpha} M$ est injective pour $\alpha<0$.

Preuve. Soient $M^{\prime}=\mathcal{H}_{X_{0}}^{0} M$ et $M^{\prime \prime}=M / M^{\prime}$, alors $M^{\prime}$ et $M^{\prime \prime}$ sont $\operatorname{des} \mathscr{D}_{X^{-}}$ Modules cohérents tels que l'action de $t$ sur $M^{\prime \prime}$ est injective. Soit $V$ la filtration induite (resp. quotient) sur $M^{\prime}$ (resp. $M^{\prime \prime}$ ), alors on vérifie que les conditions (3.1.1.1-3) sont satisfaites sauf $\left(V_{\alpha} M^{\prime}\right) t=V_{\alpha-1} M^{\prime}(\alpha<0)$; mais cela 
résulte du diagramme commutatif:

$$
\begin{aligned}
& 0 \rightarrow V_{\alpha} M^{\prime} \rightarrow V_{\alpha} M \rightarrow V_{\alpha} M^{\prime \prime} \rightarrow 0 \\
& t \downarrow \quad t \downarrow \quad t \downarrow l \\
& 0 \rightarrow V_{\alpha-1} M^{\prime} \rightarrow V_{\alpha-1} M \rightarrow V_{\alpha-1} M^{\prime \prime} \rightarrow 0 \quad(\alpha<0) \text {. }
\end{aligned}
$$

D'après 3.1 .2 et 3.1 .3 , on a $V_{\alpha} M^{\prime}=0(\alpha<0)$, donc $t: V_{\alpha} M \underset{\rightarrow}{\rightarrow} V_{\alpha-1} M(\alpha<0)$.

3.1.5. Corollaire. Soit $0 \rightarrow M^{\prime} \rightarrow M \rightarrow M^{\prime \prime} \rightarrow 0$ une suite exacte de $\mathscr{D}_{X^{-}}$ Modules coherents. Si $M$ admet la filtration rationnelle le long de $X_{0}, M^{\prime}$ et $M^{\prime \prime}$ l'admettent, de plus on a des suites exactes:

$$
0 \rightarrow V_{\alpha} M^{\prime} \rightarrow V_{\alpha} M \rightarrow V_{\alpha} M^{\prime \prime} \rightarrow 0 \quad(\alpha \in Q) .
$$

(même argument que 3.1.4).

3.1.6. Proposition. Soit $u: M \rightarrow N$ un morphisme de $\mathscr{D}_{X}$-Modules coherents qui admettent la filtration rationnelle $V$, alors $u$ preserve la filtration et est strict; en particulier, la categorie des $\mathscr{D}_{X}$-Modules coherents munis de la filtration $V$ est une categorie abelienne, dont les morphismes sont toujours stricts.

(Cela résulte de 3.1.2 et 3.1.5.)

3.1.7. Lemme. Soit $u: M \rightarrow N$ un morphisme de $\mathscr{D}_{X}$-Modules coherents munis de la filtration $V$. Soit $X^{*}=X \backslash X_{0}$. Si $\left.u\right|_{X^{*}}:\left.\left.M\right|_{X^{*} \rightarrow N}\right|_{X^{*}}$ est un isomorphisme, on a $u: V_{\alpha} M \stackrel{\rightarrow}{\rightarrow} V_{\alpha} N$ pour tout $\alpha<0$.

(Cela résulte de 3.1.3 et 3.1.6.)

3.1.8. Proposition. Soit $M$ un $\mathscr{D}_{X}$-Module coherent muni de la filtration $V$. Soit $M^{\prime}$ le plus petit sous-objet de $M$ tel que $\left.\left.M^{\prime}\right|_{X^{*}} \underset{\rightarrow}{\rightarrow} M\right|_{X^{*}}$, on a alors

$$
\begin{aligned}
& M^{\prime}=\left(V_{\alpha} M\right) \mathscr{D}_{X} \text { si } \alpha<0 \\
& M / M^{\prime} \simeq i_{*} \operatorname{Coker}\left(\partial_{t}: G r_{-1}^{V} M \rightarrow G r_{0}^{V} M\right) \\
& \mathscr{H}_{X_{0}}^{0} M \simeq i_{*} \operatorname{Ker}\left(t: G r_{0}^{V} M \rightarrow G r_{-1}^{V} M\right)
\end{aligned}
$$

ou $i: X_{0} \hookrightarrow X$ et $i_{*}$ est l'image directe de $\mathscr{D}_{X_{0}}-$ Modules (localement, $i_{*} M_{0}=M_{0} \otimes$ $\left.C\left[\partial_{t}\right]\right)$.

Preuve. Puisque $\left.\left.M^{\prime}\right|_{X^{*}} \underset{\rightarrow}{\rightarrow} M\right|_{X^{*}}$, on a $V_{\alpha} M^{\prime}=V_{\alpha} M(\alpha<0)$, donc $\left(V_{\alpha} M\right) \mathscr{D}_{X} \subset$ $M^{\prime}(\alpha<0)$. L'autre inclusion résulte de $\left(V_{\alpha} M\right) \mathscr{D}_{\left.X\right|_{X^{*}}}=\left.M_{\alpha} V\right|_{X^{*}}=\left.M\right|_{X^{*}}$ et de la définition de $M^{\prime}$. On a

$$
\begin{aligned}
G r_{0}^{V}\left(M / M^{\prime}\right) & =\operatorname{Coker}\left(G r_{0}^{V} M^{\prime} \rightarrow G r_{0}^{V} M\right) \\
& =\operatorname{Coker}\left(\partial_{t}: G r_{-1}^{V} M \rightarrow G r_{0}^{V} M\right)
\end{aligned}
$$


car $M^{\prime} \cap V_{0} M=V_{-1} M+\left(V_{-1} M\right) \partial_{t}$ (cela résulte de $M^{\prime}=\sum_{i \geq 0}\left(V_{-1} M\right) \partial_{t}^{i}$ et de $\partial_{t}^{i}$ : $V_{0} M / V_{-1} M \underset{\rightarrow}{\rightarrow} V_{i} M / V_{i-1} M$.) Puisque supp $M / M^{\prime} \subset X_{0}$, on a $M / M^{\prime}=i_{*} G r_{0}^{V}$ $\left(M / M^{\prime}\right)$, cf. 3.1.3.

D'après 3.1.3, on a $\mathcal{H}_{X_{0}}^{0} M=i_{*}(\operatorname{Ker}(t: M \rightarrow M))$, donc il suffit de montrer que $\operatorname{Ker}(t: M \rightarrow M) \subset V_{0} M$ et $\operatorname{Ker}(t: M \rightarrow M) \stackrel{\sim}{\rightarrow} \operatorname{Ker}\left(G r t: G r_{0}^{V} M \rightarrow G r_{-1}^{V} M\right)$. Soit $u \in M$ tel que $u t=0$, alors il existe $\alpha \in \boldsymbol{Q}$ tel que $u \in V_{\alpha} M$. Si $\alpha>0$, on a $u \equiv 0$ dans $G r_{\alpha}^{V} M$ car $u t \partial_{t}=0$, on a donc $u \in V_{0} M$ par récurrence. Puisque Ker $t \cap V_{\alpha} M=0(\alpha<0)$ d'après 3.1.4, Ker $t \rightarrow \operatorname{Ker} G r t$ est injectif. Si $u t \in V_{<-1} M$, i[ existe $v \in V_{<0}$ tel que $(u-v) t=0$ par la condition (3.1.1.2), donc Ker $t \rightarrow \operatorname{Ker}$ Gr $t$ est bijectif. Ce qui achève la démonstration.

\subsection{Compatibilite avec la filtration $\boldsymbol{F}$}

3.2.1. Soient $X, X_{0}, X^{*}$ et $t, \partial_{t}$ comme dans 3.1. Soit $(M, F)$ un $\mathscr{D}_{X}$-Module filtré cohérent, i.e. $G r^{F} M$ est cohérent sur $G r^{F} \mathscr{D}_{X}$. On dit que $(M, F)$ admet la filtration rationnelle $V$ le long de $X_{0}$, si:

(3.2.1.1) $M$ est muni de la filtration rationnelle de Malgrange-Kashiwara le long de $X_{0}$

(3.2.1.2) $\quad\left(F_{p} V_{\alpha} M\right) t=F_{p} V_{\alpha-1} M \quad(\alpha<0)$

(3.2.1.3) $\left(F_{p} G r_{a}^{V} M\right) \partial_{t}=F_{p+1} G r_{\alpha+1}^{V} M \quad(\alpha>-1)$

On dit que $(M, F)$ est quasi-unipotent et regulier le long de $X_{0}$, si $(M, F)$ admet la filtration rationnelle le long de $X_{0}$ et chaque $G r^{F} G r_{i}^{W} G r_{a}^{V} M$ est cohérent sur $G r^{F} \mathscr{D}_{X_{0}}$. Notons que l'action de $G r^{F} \mathscr{D}_{X_{0}}$ est bien définie sur $G r^{F} G r_{i}^{W} G r_{a}^{V} M$, où $W$ est la filtration associée à $t \partial_{t}-\alpha$ (cf. 1.3.9).

Soit $f$ une fonction holomorphe (localement définie) sur $X$. On définit $i_{f}: X \rightarrow X \times \boldsymbol{C}$ par $i_{f}(x)=(x, f(x))$, alors on dit que $(M, F)$ admet la filtration rationnelle le long de $f$, si $i_{f^{*}}(M, F)$ l'admet le long de $X \times\{0\}$, et que $(M, F)$ est quasi-unipotent et régulier le long de $f$, si $i_{f *}(M, F)$ l'est le long de $X \times\{0\}$.

3.2.2. Proposition. Avec les notations ci-dessus, on a:

(i) La condition (3.2.1.2) équivaut à:

$F_{p} V_{<0} M=V_{<0} M \cap j_{*} j^{-1} F_{p} M \quad\left(:=\left\{u \in V_{<0} M:\left.\left.u\right|_{X^{*}} \in F_{p} M\right|_{x^{*}}\right\}\right)$ pour tout $p$, où $j: X^{*} \hookrightarrow X$.

(ii) $S i\left(G r_{-1}^{V} M\right) \partial_{t}=G r_{0}^{V} M$ (i.e. $\left.M=\left(V_{<0} M\right) \mathscr{D}_{X}\right)$, la condition (3.2.1.3) pour $\alpha \geqq-1$ équivaut à:

$$
F_{p} M=\sum_{i \geq 0}\left(F_{p-i} V_{<0} M\right) \partial_{t}^{i} \quad \text { pour tout } p .
$$


(iii) Si supp $M \subset X_{0}$, la condition (3.2.1.3) équivaut à:

$$
F_{p} M=\bigoplus_{i} F_{p-i} M_{0} \otimes \partial_{t}^{i} \quad \text { pour tout } p,
$$

ou $M=M_{0} \otimes C\left[\partial_{t}\right]$ et $M_{0}=\operatorname{Ker} t\left(i . e .(M, F)=i_{*}\left(M_{0}, F\right)\right)$.

Preuve. L'implication: (3.2.2.1) $\Rightarrow(3.2 .1 .2)$ est claire, car

$$
V_{\alpha} M \cap j_{*} j^{-1} F_{p} M=\left\{u \in V_{\alpha} M:\left.\left.u\right|_{X^{*}} \in F_{p} M\right|_{X^{*}}\right\}
$$

(3.2.1.2) $\Rightarrow$ (3.2.2.1): Soit $u \in\left(j_{*} j^{-1} F_{p} M\right) \cap V_{<0} M$, alors il existe (localement) $i \geqslant 0$ tel que $u t^{i} \in F_{p} M$, i.e. $u t^{i} \in F_{p} V_{<i} M$, donc $u \in F_{p} V_{<0} M$.

(3.2.1.3) pour $\alpha \geq-1 \Leftrightarrow(3.2 .2 .2)$ : On définit la filtration $F^{\prime}$ par

$$
F_{p}^{\prime} M=\sum_{i \geqslant 0}\left(F_{p-i} V_{<0} M\right) \partial_{t}^{i} .
$$

D'abord admettons les assertions:

$$
F_{p}^{\prime} V_{<j} M=\sum_{0 \leqslant i \leqslant j}\left(F_{p-i} V_{<0} M\right) \partial_{t}^{i} \quad(j \geqslant 0)
$$

$$
F_{p}^{\prime} V_{\alpha} M=\sum_{0 \leqslant i \leqslant[\alpha]}\left(F_{p-i} V_{<0} M\right) \partial_{t}^{i}+\left(F_{p-[\alpha]-1} V_{\alpha-[\alpha]-1} M\right) \partial_{t}^{[\alpha]+1} \quad(\alpha \geqslant 0) .
$$

On a alors

et

$$
G r_{\alpha}^{V} F_{p}^{\prime} M=\left(G r_{\alpha-[\alpha]-1}^{V} F_{p-[\alpha]-1} M\right) \partial_{t}^{[\alpha]+1} \quad(\alpha \geqslant 0)
$$

$$
F_{p}^{\prime} V_{<0} M=F_{p} V_{<0} M
$$

Puisque $F_{p}^{\prime} M \subset F_{p} M$, on a $F_{p}^{\prime} M=F_{p} M$ pour tout $p$, sii

$$
G r_{a}^{V} F_{p} M=\left(G r_{a-[\alpha]-1}^{V} F_{p-[\alpha]-1} M\right) \partial_{t}^{[\alpha]+1} \text { pour tout } p \in \mathbb{Z}, \alpha \geqslant 0,
$$

i.e.

$$
G r_{a}^{V} F_{p} M=\left(G r_{\alpha-1}^{V} F_{p-1} M\right) \partial_{t} \text { pour tout } p \in \mathbb{Z}, \alpha \geqslant 0 ;
$$

d'où l'assertion.

Maintenant démontrons (3.2.2.4-5). Puisque $\partial_{t}^{j}: G r_{a-j}^{V} M \rightarrow G r_{a}^{V} M$ est injectif pour $\alpha-j>-1$, on a

$$
\left(F_{p-[\alpha]-1} V_{<0} M\right) \partial_{j}^{[\alpha]+1} \cap V_{\alpha} M=\left(F_{p-[\alpha]-1} V_{\alpha-[\alpha]-1} M\right) \partial_{t}^{[\alpha]+1} \quad(\alpha \geqslant 0)
$$

donc (3.2.2.5) est réduit à (3.2.2.4). Pour démontrer (3.2.2.4), il suffit de vérifier

$$
\left(F_{p-j-1} V_{<0} M\right) \partial_{t}^{j+1} \cap V_{<j} M \subset\left(F_{p-j} V_{<0} M\right) \partial_{t}^{j} .
$$

Si $u \in F_{p-j-1} V_{<0} M$ satisfait $u \partial_{t}^{j+1} \in V_{<j} M$, on a $u \partial_{t} \in V_{<0} M$, car

$$
\partial_{t}^{j}: V_{<1} M / V_{<0} M \rightarrow V_{<j+1} M / V_{<j} M
$$


est injectif; on a donc $u \partial_{t}^{j+1} \in\left(F_{p-j} V_{<0} M\right) \partial_{t}^{j}$; d'où l'assertion.

(3.2.1.3) $\Leftrightarrow(3.2 .2 .3)$ : D'après 3.1 .3 , on a

$$
M=M_{0} \otimes C\left[\partial_{t}\right], \quad V_{\alpha} M=\bigoplus_{0 \leqslant i \leqslant[\alpha]} M_{0} \otimes \partial_{t}^{i} .
$$

Posons $F_{p}^{\prime} M=\bigoplus_{i} F_{p-i} M_{0} \otimes \partial_{t}^{i}$, où $F_{p} M_{0}=M_{0} \cap F_{p} M$. Puisque $F_{p}^{\prime} M \subset F_{p} M$, $F_{p}^{\prime} V_{0} M=F_{p} V_{0} M$ et $F_{p}^{\prime} G r_{i}^{V} M=\left(F_{p-i} G r_{0}^{V} M\right) \partial_{t}^{i}(p \in Z, i \geqslant 0)$, on a $F^{\prime}=F$, sii $F_{p} G r_{i}^{V} M=\left(F_{p-i} G r_{0}^{V} M\right) \partial_{t}^{i}$, i.e. $\left(F_{p} G r_{i}^{V} M\right) \partial_{t}=F_{p+1} G r_{i+1}^{V} M$ pour tout $p \in \boldsymbol{Z}$, $i \geqslant 0$. Ce qui achève la démonstration de 3.2.2.

3.2.3. Remarque. $\mathrm{Si}\left(G r_{-1}^{V} M\right) \partial_{t}=G r_{0}^{V} M, 3.2 .2$ implique que les conditions (3.2.1.2) pour $\alpha<0$ et (3.2.1.3) pour $\alpha \geq-1$ sont satisfaites, sii:

$$
F_{p} M=\sum\left(V_{<0} M \cap j_{*} j^{*} F_{p-i} M\right) \partial_{t}^{i} \text { pour tout } p .
$$

Si $G r t: G r_{0}^{V} M \rightarrow G r_{-1}^{V} M$ est injectif, on a

$$
F_{p} M=\Sigma\left(V_{0} M \cap j_{*} j^{*} F_{p-i} M\right) \partial_{t}^{i} \text { pour tout } p,
$$

sii on a (3.2.1.2-3) et, de plus, $G r t:\left(G r_{0}^{V} M, F\right) \rightarrow\left(G r_{-1}^{V} M, F\right)$ est strict. En fait, on a (3.2.2.1) (resp. (3.2.2.2)) en remplacant $V_{<0}$ par $V_{0}$, sii on a (3.2.1.2) et $G r t$ set strict (resp. sii on a (3.2.1.3)).

3.2.4. Lemme. Soient $i: X \hookrightarrow Y$ une immersion fermée et $t: Y \rightarrow C$ un morphisme lisse tels que $t i: X \rightarrow C$ soit lisse. Posons $Y_{0}=t^{-1}(0), X_{0}=i^{-1} Y_{0}$, alors un $\mathscr{D}_{X}$-Module filtré cohérent $(M, F)$ admet la filtration rationnelle le long de $X_{0}$, sii $(\tilde{M}, F)=i_{*}(M, F)$ l'admet le long de $Y_{0}$; dans ce cas, on a $(\tilde{M}, F, V)=$ $i_{*}(M, F, V)$, i.e. localement

$$
\tilde{M}=M \otimes C\left[\partial^{\prime}\right], \quad F_{p} \tilde{M}=\oplus F_{p-|\nu|} M \otimes \partial^{\prime \nu}, \quad V_{\alpha} \tilde{M}=V_{\alpha} M \otimes C\left[\partial^{\prime}\right]
$$

ou $\left(t, x_{1}, \cdots, x_{n}\right)$ sont des coordonnees de $Y$ telles que

$$
X=\left\{x_{1}=\cdots=x_{l}=0\right\} \quad \text { et } \quad \partial^{\prime}=\left(\partial_{x_{1}}, \cdots, \partial_{x_{l}}\right) .
$$

Preuve. Puisque l'assertion est locale, on peut supposer que $\operatorname{codim}_{Y} X=1$, i.e. $X=\{x=0\}$, alors $\tilde{M}=M\left[\partial_{x}\right], F_{p} \tilde{M}=\oplus F_{p-i} M \otimes \partial_{x}^{i}$ avec $M \otimes \partial_{x}^{i}=\mathrm{Ker}$ $\left(x \partial_{x}-i\right) \subset \tilde{M}$. Si $\tilde{M}$ admet la filtration $V$, chaque $V_{\alpha} \tilde{M}$ est stable par l'action de $x \partial_{x}$, car $x \partial_{x} \in V_{0} \mathscr{D}_{Y}$; donc $V_{\alpha} \tilde{M}$ est compatible avec la décomposition $M=$ $\bigoplus_{i} M \otimes \partial_{x}^{i}$, et on obtient une filtration de $M$ qui satisfait les conditions (3.1.1.1-3) car on peut choisir $\partial_{t}$ et $\partial_{x}$ tels que $\left[\partial_{t}, x \partial_{x}\right]=0,\left[t, x \partial_{x}\right]=0$ (e.g. on prend des coordonnées $\left.\left(t, x_{1}, x_{2}, \cdots, x_{m}\right)\right)$. Si $(M, F)$ admet la filtration $V$, alors $V_{\alpha} \tilde{M}=$ $V_{\alpha} M\left[\partial_{x}\right]$ satisfait les conditions (3.1.1.1-3). On vérifie facilement que $(M, F, V)$ 
satisfait les conditions (3.2.1.1.-3), sii $i_{*}(M, F, V)$ les satisfait.

3.2.5. Corollaire. Soient $(M, F), f, i_{f}$ et $(\tilde{M}, F)=i_{f^{*}}(M, F)$ comme dans 3.2.1. Supposons que $f: X \rightarrow C$ soit lisse, alors $(M, F)$ admet la filtration rationnelle le long de $f$, sii $(M, F)$ l'admet le long de $X_{0}:=f^{-1}(0)$; dans ce cas, oú $a$

$$
i_{*}\left(G r_{a}^{V} M, F\right)=\left(G r_{a}^{V} \tilde{M}, F\right)
$$

ou $i: X_{0} \hookrightarrow X$.

(C'est clair, car $t: X \times C \rightarrow C$ et $f=t \circ i_{f}: X \rightarrow C$ sont lisses.)

3.2.6. Lemme. Soient $(M, F)$, $f$ et $i_{f}$ comme dans 3.2.1. Posons $i_{0}: X=$ $X \times\{0\} \rightarrow X \times C$. Supposons que supp $M \subset f^{-1}(0)$, alors les conditions suivantes sont equivalentes:

$$
\left(F_{p} M\right) f \subset F_{p-1} M \text { pour tout } p,
$$

$$
(M, F) \text { est quasi-unipotent et régulier le long de } f \text {, }
$$$$
\text { on a l'isomorphisme canonique: } i_{f}(M, F) \underset{\rightarrow}{\sim} i_{0^{*}}(M, F) \text {. }
$$

Preuve. Posons $(\tilde{M}, F)=i_{f^{*}}(M, F)$, on a par définition une decomposition: $\tilde{M}=\oplus M \otimes \partial_{t}^{i}$ telle que $M \otimes \partial_{t}^{i}=\operatorname{Ker}\left((t-f) \partial_{t}-i\right)$ et que $F_{p} \tilde{M}=\oplus F_{p-i} M \otimes \partial_{t}^{i}$. D'après 3.1.3, on a une autre: $\tilde{M}=\oplus \tilde{M}_{0} \otimes \partial_{t}^{i}$ telle que $\tilde{M}_{0} \otimes \partial_{t}^{i}=\operatorname{Ker}\left(t \partial_{t}-i\right)$ et que $V_{j} \tilde{M}=\bigoplus_{0 \leq i \leq j} \tilde{M}_{0} \otimes \partial_{t}^{i}$. Puisque

$$
\left(\sum u_{j} \otimes \partial_{t}^{j}\right)\left(t \partial_{t}-i\right)=\sum\left(u_{j-1} f+u_{j}(j-i)\right) \otimes \partial_{t}^{j} \text { pour } u_{j} \in M,
$$

on a $\tilde{M}_{0} \otimes \partial_{t}^{j}=\left\{\sum u(-f)^{i} / i ! \otimes \partial_{t}^{i+j}: u \in M\right\}$, et on obtient l'isomorphisme canonique (3.2.6.3) sans filtration $F$, par l'isomorphisme $\tilde{M}_{0} \otimes 1 \underset{\rightarrow}{\rightarrow}$ induit par la projection $\tilde{M} \rightarrow M \otimes 1$. On a (3.2.6.2) $\Rightarrow(3.2 .6 .1)$ d'après 3.2.2, car la compatibilité de la decomposition: $\tilde{M}=\oplus \tilde{M}_{0} \otimes \partial_{t}^{i}$ avec la filtration $F$ implique:

$$
F_{p} \tilde{M} \cap\left(\tilde{M}_{0} \otimes \partial_{t}^{j}\right)=\left\{\sum u(-f)^{i} / i ! \otimes \partial_{t}^{i+j} \mid u \in F_{p-j} M\right\},
$$

par le morphisme: $M \otimes \partial_{t}^{j} \rightarrow \tilde{M} \rightarrow \tilde{M}_{0} \otimes \partial_{t}^{j}$. De Même on vérifie que (3.2.6.1) implique la compatibilité ci-dessus et la condition (3.2.6.4); d'où (3.2.6.1) $\Rightarrow$ (3.2.6.3). L'implication (3.2.6.3) $\Rightarrow(3.2 .6 .2)$ est triviale; d'où l'assertion.

3.2.7. Lemme. Soient $X, X_{0}$ et $(M, F)$ comme dans 3.2.1. Supposons que $M$ (resp. $G r_{p}^{F} M \otimes_{\mathcal{O}_{X}} \mathcal{O}_{X_{0}}$ ) est localement libre de type fini sur $\mathcal{O}_{X}$ (resp. $\mathcal{O}_{X_{0}}$ ) et que $(M, F)$ admet la filtration rationnelle le long de $X_{0}$, alors chaque $G r_{p}^{F} M$ est localement libre de type fini sur $\mathcal{O}_{X}$ dans un voisinage de $X_{0}$. 
Preuve. Par hypothèse, on a $V_{-i-1} M=M t^{i}, F_{p} V_{-i-1} M=\left(F_{p} M\right) t^{i}(i \geqslant 0)$, donc

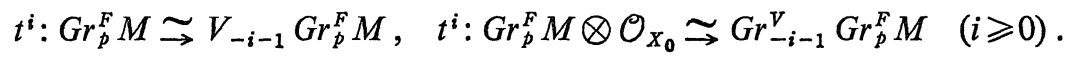

Puisque $G r_{p}^{F} M \otimes \mathcal{O}_{X_{0}}$ est localement libre, on a localement un isomorphisme

$$
\lim _{\leftarrow} G r_{p}^{F} M /\left(G r_{p}^{F} M\right) t^{i} \simeq\left(G r_{p}^{F} M{\underset{\mathcal{O}}{X}}_{\mathcal{O}_{X_{0}}}\right)[[t]] \simeq\left(\oplus \mathcal{O}_{X_{0}}\right)[[t]]
$$

(non canoniquement). Puisque $G r_{p}^{F} M$ est cohérent, $G r_{p}^{F} M$ est localement libre de type fini $\operatorname{sur} \mathcal{O}_{X}$ dans un voisinage de $X_{0}$.

\subsection{Categorie derivées et Images directes}

3.3.1. Soient $X, X_{0}, t, \partial_{t}$ et $\left(\mathscr{D}_{X}, F, V\right)$ comme dans 3.1 et 3.2 . Soit $M F V\left(\mathscr{D}_{X}\right)$ la catégorie additive des $\mathscr{D}_{X}$-Modules filtrés $(M, F, V)$ qui satisfaient les conditions:

$$
\begin{gathered}
\bigcup_{p \in Z} F_{p} M=\bigcup_{\alpha \in \boldsymbol{Q}} V_{\alpha} M=M, F_{p} M=0 \quad \text { pour } p \ll 0 \quad \text { (localement) } \\
\left(F_{p} V_{\alpha} M\right)\left(F_{p} V_{\beta} \mathscr{D}_{X}\right) \subset F_{p+q} V_{\alpha+\beta} M \quad(p, q \in \boldsymbol{Z}, \alpha, \beta \in \boldsymbol{Q}), \\
\begin{cases}t: F_{p} V_{\alpha} M \underset{\sim}{\longrightarrow} F_{p} V_{\alpha-1} M \quad(\alpha<0), \\
\partial_{t}: F_{p} G r_{\alpha}^{V} M \underset{\rightarrow}{\rightarrow} F_{p+1} G r_{\alpha+1}^{V} M \quad(\alpha>-1),\end{cases}
\end{gathered}
$$

où les morphismes sont $\mathscr{D}_{X}$-linéaire et préservent les filtrations.

Soient $C F V\left(\mathscr{D}_{X}\right)=C\left(M F V\left(\mathscr{D}_{X}\right)\right), K F V\left(\mathscr{D}_{X}\right)=K\left(M F V\left(\mathscr{D}_{X}\right)\right)$ comme dans [31], où on suppose que $V_{\alpha} M^{*}=V_{[m a] / m} M^{*}$ pour quelque $m>0$, si $\left(M^{*}, F, V\right) \in$ $\operatorname{CFV}\left(\mathscr{D}_{X}\right)$. On définit la sous-catégorie pleine $K F V\left(\mathscr{D}_{X}\right)^{\emptyset}$ par:

$$
\left(M^{*}, F, V\right) \in K F V\left(\mathscr{D}_{X}\right)^{\emptyset} \text { sii } H^{i}\left(F_{p} V_{\alpha} M^{*}\right)=0 \text { pour tout } i, p, \alpha .
$$

Posons $\operatorname{DFV}\left(\mathscr{D}_{X}\right)=K F V\left(\mathscr{D}_{X}\right) / K F V\left(\mathscr{D}_{X}\right)^{\emptyset}$.

On définit $D^{*} F V\left(\mathscr{D}_{X}\right)$ pour $*=+,-, b$, comme dans [31], alors ce sont des sous-catégories pleines de $\operatorname{DFV}\left(\mathscr{D}_{X}\right)$, cf. la preuve de 1.2.4.

Considérons les conditions suivantes:

(3.3.1.4) l'action de $t \partial_{t}-\alpha \operatorname{sur} \mathscr{H}^{i} G r_{\alpha}^{V} M^{\bullet}$ est nilpotente $(i \in \mathbb{Z}, \alpha \in \boldsymbol{Q})$

(3.3.1.5) chaque $\mathscr{H}^{i} F_{p} V_{\alpha} M^{\bullet}$ est cohérent $\operatorname{sur} \mathcal{O}_{X} \quad(i \in \boldsymbol{Z}, p \in \boldsymbol{Z}, \alpha \in \boldsymbol{Q})$.

On définit la sous-catégorie pleine $D F V_{m}\left(\mathscr{D}_{X}\right)$ (resp. $\left.D F V_{c}\left(\mathscr{D}_{X}\right)\right)$ par: $\left(M^{*}, F, V\right)$ $\in D F V_{m}\left(\mathscr{D}_{X}\right)$ (resp. $\left.D F V_{c}\left(\mathscr{D}_{X}\right)\right)$ sii $(M, F, V)$ satisfait la condition (3.3.1.4) (resp. (3.3.1.5)). Posons $D F V_{m c}\left(\mathscr{D}_{X}\right)=D F V_{m}\left(\mathscr{D}_{X}\right) \cap D F V_{c}\left(\mathscr{D}_{X}\right)$.

3.3.2. Lemme. Soit $(M, F, V) \in M F V\left(\mathscr{D}_{X}\right)$, posons 


$$
\begin{array}{ll}
\left(F_{p} V_{\alpha} M\right)^{\wedge}=\lim _{\stackrel{\leftarrow}{\kappa}} F_{p}\left(V_{\alpha} / V_{\beta}\right) M, & F_{p} \hat{M}=\lim _{\vec{a}}\left(F_{p} V_{\alpha} M\right)^{\wedge} \\
V_{\alpha} \hat{M}=\lim _{\vec{p}}\left(F_{p} V_{\alpha} M\right)^{\wedge} & , \hat{M}=\lim _{\overrightarrow{a p}}\left(F_{p} V_{\alpha} M\right)^{\wedge},
\end{array}
$$

alors les morphismes canoniques $F_{p} \hat{M} \rightarrow \hat{M}, V_{\alpha} \hat{M} \rightarrow \hat{M}$ et $\left(F_{p} V_{\alpha} M\right)^{\wedge} \rightarrow \hat{M}$ sont injectifs, et $F_{p} V_{\alpha} \hat{M}=\left(F_{p} V_{\alpha} M\right)^{\wedge}$ ou $F_{p} V_{\alpha} \hat{M}=F_{p} \hat{M} \cap V_{\alpha} \hat{M} ;$ de plus, $F_{p}\left(V_{\alpha} / V_{\beta}\right) \hat{M} \stackrel{\longrightarrow}{\longrightarrow}$ $F_{p}\left(V_{\alpha} / V_{\beta}\right) M . \quad\left(\right.$ On notera $(\hat{M}, F, V)=(M, F, V)^{\wedge}$.)

Preuve. Pour $p>q, \alpha>\beta>\gamma$, on a un diagramme commutatif

$$
\begin{gathered}
0 \rightarrow F_{q}\left(V_{\beta} / V_{\gamma}\right) M \rightarrow F_{q}\left(V_{\alpha} / V_{\gamma}\right) M \rightarrow F_{q}\left(V_{\alpha} / V_{\beta}\right) M \rightarrow 0 \\
\downarrow \\
\begin{array}{c}
2 \\
0
\end{array} F_{p}\left(V_{\beta} / V_{\gamma}\right) M \rightarrow F_{p}\left(V_{\alpha} / V_{\gamma}\right) M \rightarrow F_{p}\left(V_{\alpha} / V_{\beta}\right) M \rightarrow 0 .
\end{gathered}
$$

Par la condition de Mittag-Leffler [38], on obtient:

$$
\begin{aligned}
& 0 \rightarrow\left(F_{q} V_{\beta} M\right)^{\wedge} \rightarrow\left(F_{q} V_{\alpha} M\right)^{\wedge} \rightarrow F_{q}\left(V_{\alpha} / V_{\beta}\right) M \rightarrow 0 \\
& 0 \rightarrow\left(F_{p} V_{\beta} M\right)^{\wedge} \rightarrow\left(F_{p} V_{\alpha} M\right)^{\wedge} \rightarrow F_{p}\left(V_{\alpha} / V_{\beta}\right) M \rightarrow 0
\end{aligned}
$$

donc $\left(F_{q} V_{\beta} M\right)^{\wedge} \rightarrow \hat{M}$ sont injectifs et $F_{q} V_{\beta} \hat{M}=\left(F_{q} V_{\beta} M\right)^{\wedge}$ (en prenant la limit $\alpha \rightarrow \infty, p \rightarrow \infty)$; d'où l'assertion.

3.3.3. Lemme. Soit $\left(M^{\circ}, F, V\right) \in D F V_{m}\left(\mathscr{D}_{X}\right)$. Si chaque $\left(G r_{a}^{V} M, F\right)$ est strict, $\left(\hat{M}^{\bullet}, F, V\right)$ est strict, i.e. $\mathscr{H}^{i}\left(F_{p} V_{\alpha} \hat{M}\right) \rightarrow \mathcal{H}^{i}(\hat{M})$ sont injectifs et $\mathcal{H}^{i}\left(F_{p} \hat{M}\right) \cap$ $\mathscr{H}^{i}\left(V_{\alpha} \hat{M}\right)=\mathscr{H}^{i}\left(F_{p} V_{\alpha} \hat{M}\right)$ pour tout $i, p, \alpha, c f .1 .2$.

Preuve. Pour $\alpha>\beta>\gamma$, on a une suite exacte scindée de $V_{0} \mathscr{D}_{X}$-Modules

$$
0 \rightarrow \mathscr{H}^{i}\left(V_{\beta} / V_{\gamma}\right) M^{\bullet} \rightarrow \mathscr{H}^{i}\left(V_{\alpha} / V_{\gamma}\right) M^{\bullet} \rightarrow \mathscr{H}^{i}\left(V_{\alpha} / V_{\beta}\right) M^{\bullet} \rightarrow 0,
$$

en particulier, $\left(\left(V_{\alpha} / V_{\beta}\right) M^{\circ}, V\right)$ est strict, donc $\left(\left(V_{\alpha} / V_{\beta}\right) M^{\circ}, F, V\right)$ est strict, car chaque $\left(G r_{\alpha}^{V} M, F\right)$ l'est, cf. 1.2.9(d); alors l'assertion résulte du lemme suivant:

3.3.4. Lemme. Soit $\left(M^{\circ}, F, V\right) \in D F V\left(\mathscr{D}_{X}\right)$, alors $\left(\left(V_{\alpha} / V_{\beta}\right) M^{\circ}, F, V\right)$ est strict pour tout $\alpha>\beta$, sii $\left(\hat{M}^{\circ}, F, V\right)=\left(M^{\circ}, F, V\right)^{\wedge}$ est strict; dans ce cas, $\mathscr{H}^{i} F_{p} V_{\alpha} \hat{M}^{0}=\lim _{\leftarrow} \mathcal{H}^{i} F_{p}\left(V_{\alpha} / V_{\beta}\right) M^{\bullet}$

Preuve. Supposons que $\left(\left(V_{\alpha} / V_{\beta}\right) M^{\circ}, F, V\right)$ sont stricts, alors $\mathscr{H}^{i} F_{p}\left(V_{\beta} / V_{\gamma}\right) M^{\circ}$ $\rightarrow \mathcal{H}^{i} F_{p}\left(V_{\alpha} / V_{\gamma}\right) M^{\bullet}$ est injectif, donc on obtient la suite exacte

$$
0 \rightarrow \mathcal{H}^{i} F_{p}\left(V_{\beta} / V_{\gamma}\right) M^{\bullet} \rightarrow \mathcal{H}^{i} F_{p}\left(V_{\alpha} / V_{\gamma}\right) M^{\bullet} \rightarrow \mathscr{H}^{i} F_{p}\left(V_{\alpha} / V_{\beta}\right) M^{\bullet} \rightarrow 0 ;
$$

en particulier, la condition de Mittag-Leffler est satisfaite pour $\left\{\mathcal{H}^{i} F_{p}\left(V_{\alpha} / V_{\beta}\right) M^{\circ}\right\}_{\beta}$, 

donc $\mathscr{H}^{i} F_{p} V_{\alpha} \hat{M}^{\cdot}=\lim _{\leftarrow} \mathscr{H}^{i} F_{p}\left(V_{\alpha} / V_{\beta}\right) M^{*} ;$ par le même argument que dans 3.3.2,
on a

$$
\begin{aligned}
& \begin{array}{c}
0 \rightarrow \mathcal{H}^{i} F_{q} V_{\beta} \hat{M}^{\cdot} \\
\eta
\end{array} \mathscr{H}^{i} F_{q} V_{\alpha} \hat{M}^{\cdot} \rightarrow \mathscr{H}^{i} F_{q}\left(V_{\alpha} / V_{\beta}\right) M^{\bullet} \rightarrow 0 \\
& 0 \rightarrow \mathscr{H}^{i} F_{p} V_{\beta} \hat{M}^{\bullet} \rightarrow \mathscr{H}^{i} F_{p} V_{\alpha} \hat{M}^{\bullet} \rightarrow \mathcal{H}^{i} F_{p}\left(V_{\alpha} / V_{\beta}\right) M^{\bullet} \rightarrow 0
\end{aligned}
$$

i.e. $\left(\hat{M}^{\bullet}, F, V\right)$ est strict.

Si $\left(\hat{M}^{*}, F, V\right)$ est strict, $\left(\left(V_{\alpha} / V_{\beta}\right) \hat{M}^{\bullet}, F, V\right)$ sont stricts, cf. 1.2.9. Puisque $\left(\left(V_{\alpha} / V_{\beta}\right) M^{*}, F, V\right) \underset{\rightarrow}{\rightarrow}\left(\left(V_{\alpha} / V_{\beta}\right) \hat{M}^{*}, F, V\right)$, on obtient l'assertion. Ce qui achève la démonstration de 3.3.4 et de 3.3.3.

3.3.5. Lemme. Soit $\left(M^{*}, F, V\right) \in D F V_{c}\left(\mathscr{D}_{X}\right)$. Si $\left(M^{*}, F, V\right)^{\wedge}$ est strict, et si $\mathscr{H}^{i} F_{p} G r_{a}^{V} M^{\bullet}=0$ pour $i \gg 0$, alors $\left.\left(M^{\bullet}, F, V\right)\right|_{x_{0}}$ est strict.

Preuve. Soit $\alpha<0$. Puisque $\left(\hat{M}^{\bullet}, V\right)$ est strict et $t: V_{\alpha} \hat{M}^{\bullet} \rightarrow V_{\alpha-1} \hat{M}^{\bullet}, \mathscr{H}^{i} V_{\alpha} \hat{M}^{\bullet}$ est sans $t$-torsion, donc $\mathscr{H}^{i} F_{p} V_{\alpha} M^{\bullet}$ est aussi sans $t$-torsion, si $\mathscr{H}^{i} F_{p} V_{\alpha} M^{\prime \bullet} \rightarrow$ $\mathscr{H}^{i} F_{p} V_{\alpha} \hat{M}^{*}$ est injectif, où $M^{\prime \prime}=\left.M^{*}\right|_{x_{0}}$. On va démontrer l'assertion:

$$
\mathscr{H}^{i} F_{p} V_{\alpha} \hat{M}^{\bullet}=\lim _{\leftarrow} \mathcal{H}^{i} F_{p} V_{\alpha} M^{\bullet} /\left(\mathcal{H}^{i} F_{p} V_{\alpha} M^{*}\right) t^{j} \quad(\alpha<0)
$$

par récurrence sur $i$, car (3.3.5.1) entraine l'injectivité de $\mathscr{H}^{i} F_{p} V_{\alpha} M^{\prime \bullet} \rightarrow$ $\mathcal{H}^{i} F_{p} V_{\alpha} \hat{M}^{\bullet}(\alpha<0)$. Notons que (3.3.5.1) est vérifiée pour $i \gg 0$, car $\mathscr{H}^{i} F_{p} V_{\alpha} M^{\prime \bullet}$ $=0(i \gg 0)$ résulte de la suite exacte:

$$
\begin{aligned}
& \rightarrow \mathcal{H}^{i} F_{p} V_{\alpha} M^{\cdot} \stackrel{t^{j}}{\rightarrow} \mathcal{H}^{i} F_{p} V_{\alpha} M^{\cdot} \rightarrow \mathcal{H}^{i} F_{p}\left(V_{\alpha} / V_{\alpha-j}\right) M^{\cdot} \\
& \rightarrow \mathcal{H}^{i+1} F_{p} V_{\alpha} M^{\cdot} \stackrel{t^{j}}{\rightarrow} \mathcal{H}^{i+1} F_{p} V_{\alpha} M^{*} \rightarrow .
\end{aligned}
$$

Supposons que (3.3.5.1) est vérifiée pour $i+1$, alors on déduit $\mathscr{H}^{i} F_{p}\left(V_{\alpha /} / V_{\alpha-j}\right) M^{\bullet}$ $=\mathscr{H}^{i} F_{p} V_{\alpha} M^{*} /\left(\mathcal{H}^{i} F_{p} V_{\alpha} M^{*}\right) t^{j}$ de la suite exacte ci-dessus; donc on a (3.3.5.1) pour $i$, cf. 3.3.4.

Pour $\alpha>\beta, i, p \in \boldsymbol{Z}$, considérons le diagramme commutatif:

$$
\begin{aligned}
& 0 \rightarrow \mathscr{H}^{i} F_{p} V_{\beta} M^{\prime \bullet} \stackrel{v}{\rightarrow} \mathcal{H}^{i} F_{p} V_{\alpha} M^{\prime \bullet} \rightarrow \mathscr{H}^{i} F_{p}\left(V_{\alpha} / V_{\beta}\right) M^{\prime \bullet} \rightarrow 0 \\
& u^{\prime} \downarrow \quad u \downarrow \quad \downarrow \\
& 0 \rightarrow \mathscr{H}^{i} F_{p} V_{\beta} \hat{M}^{\bullet} \rightarrow \mathcal{H}^{i} F_{p} V_{\alpha} \hat{M}^{\bullet} \rightarrow \mathcal{H}^{i} F_{p}\left(V_{\alpha} / V_{\beta}\right) \hat{M}^{\bullet} \rightarrow 0 \text {. }
\end{aligned}
$$

Par hypothèse, la deuxième ligne est exacte. Si $\beta<0$, on a démontré que $u^{\prime}$ est injectif, donc $v$ est injectif par le diagramme, et la première ligne est exacte $(\beta<0)$; alors $u$ est aussi injectif, donc, par le même argument, la première ligne est exacte pour tout $\alpha>\beta, i, p \in Z$. Puisque $\mathscr{H}^{i} F_{q} V_{\alpha} \hat{M}^{*} \rightarrow \mathscr{H}^{i} F_{p} V_{\alpha} \hat{M}^{\bullet}$ sont in- 
jectifs, $\mathscr{H}^{i} F_{q} V_{\alpha} M^{\prime \circ} \rightarrow \mathscr{H}^{i} F_{p} V_{\alpha} M^{\prime \circ}$ sont aussi injectifs; on a donc obtenu le diagramme commutatif:

$$
\begin{aligned}
& 0 \rightarrow \mathscr{H}^{i} F_{q} V_{\beta} M^{\prime \bullet} \rightarrow \mathcal{H}^{i} F_{q} V_{\alpha} M^{\prime \bullet} \rightarrow \mathscr{H}^{i} F_{q}\left(V_{\alpha} / V_{\beta}\right) M^{\circ} \rightarrow 0 \\
& 2 \quad 2 \\
& 0 \rightarrow \mathscr{H}^{i} F_{p} V_{\beta} M^{\prime \bullet} \rightarrow \mathcal{H}^{i} F_{p} V_{\alpha} M^{\prime \prime} \rightarrow \mathcal{H}^{i} F_{p}\left(V_{\alpha} / V_{\beta}\right) M^{\circ} \rightarrow 0
\end{aligned}
$$

d'où l'assertion.

3.3.6. Définition. Soient $X, X_{0}$ et $\left(\mathscr{D}_{X}, F, V\right)$ comme dans 3.3.1. Soient $\alpha \in \boldsymbol{Q}$ $\cap[-1,0]$ et $p \in \mathbb{Z}$. Soit $L$ un $\mathcal{O}_{X}$-Module tel que $\bigcup_{i} \operatorname{Ker}\left(t^{i}: L \rightarrow L\right)=\operatorname{Ker}$ $(t: L \rightarrow L)$ si $\alpha=0$ et $L$ est sans $t$-torsion si $\alpha \neq 0$. On définit $L \bigotimes_{O_{X}}\left(\mathscr{D}_{X}, F[p]\right.$, $V[\alpha])=(M, F, V)$ par:

$$
\begin{aligned}
M=L \bigotimes_{\mathcal{O}_{X}} \mathscr{D}_{X}, F_{q} M & =\operatorname{Im}\left(L \otimes F_{q-p} \mathscr{D}_{X} \hookrightarrow L \otimes \mathscr{D}_{X}\right) \\
V_{\beta} M & =\operatorname{Im}\left(L \otimes V_{\beta-\alpha} \mathscr{D}_{X} \rightarrow L \otimes \mathscr{D}_{X}\right) .
\end{aligned}
$$

3.3.7. Lemme. $\quad F_{q} V_{\beta} M=\operatorname{Im}\left(L \otimes F_{q-p} V_{\beta-\alpha} \mathscr{D}_{X} \rightarrow L \otimes \mathscr{D}_{X}\right)$ et $L \otimes\left(\mathscr{D}_{X}\right.$, $F[p], V[\alpha]) \in M F V\left(\mathscr{D}_{X}\right)(-1 \leqslant \alpha \leqslant 0)$.

Preuve. On peut supposer que $p=0$. Si $L$ est sans $t$-torsion, alors $L \otimes F_{q} V_{\beta-\alpha} \mathscr{D}_{X}$ $\rightarrow L \otimes \mathscr{D}_{X}$ sont injectifs et l'assertion est claire, car $\operatorname{Tor}_{1}^{\mathcal{O}_{X}}(L, N)=0$ pour $N=\mathscr{D}_{X} / F_{q} V_{\beta} \mathscr{D}_{X}, F_{q} \mathscr{D}_{X} / F_{q} V_{\beta} \mathscr{D}_{X}$, etc. Si $L t=0$ et $\alpha=0$, on a

$$
\begin{aligned}
& L \otimes \mathscr{D}_{X}=L \otimes_{O_{X_{0}}} \mathscr{D}_{X_{0}}\left[\partial_{t}\right] \\
& F_{q}\left(L \otimes \mathscr{D}_{X}\right)=L \otimes_{\mathcal{O}_{X_{0}}}\left(\bigoplus_{i} F_{q-i} \mathscr{D}_{X_{0}} \otimes \partial_{t}^{i}\right) \\
& \left.V_{\beta}\left(L \otimes \mathscr{D}_{X}\right)=L{\underset{O_{X_{0}}}{0 \leqslant i \leqslant[\beta]}}_{0} \mathscr{D}_{X_{0}} \otimes \partial_{i}^{i}\right) \text {. } \\
& \operatorname{Im}\left(L \otimes F_{q} V_{\beta} \mathscr{D}_{X} \rightarrow L \otimes \mathscr{D}_{X}\right)=L \mathcal{O}_{X_{0}}\left(\underset{0 \leqslant i \leqslant[\beta]}{\bigoplus_{q-i}} F_{x^{\prime}} \mathscr{D}_{X_{0}} \otimes \partial_{t}^{i}\right)
\end{aligned}
$$

d'où l'assertion. Considérons le cas $\alpha=0$. Par hypothèse, on a une suite exacte $0 \rightarrow L^{\prime} \rightarrow L \rightarrow L^{\prime \prime} \rightarrow 0$ telle que $L^{\prime} t=0$ et $L^{\prime}$ est sans $t$-torsion. On définit $L_{q \beta}^{\prime}, L_{q \beta}$ et $L_{q \beta}^{\prime \prime}$ par

$$
L_{q \beta}=\operatorname{Im}\left(L \otimes F_{q} V_{\beta} \mathscr{D}_{X} \rightarrow L \otimes \mathscr{D}_{X}\right), \text { etc. }
$$

On a une suite exacte $0 \rightarrow L_{q \beta}^{\prime} \rightarrow L_{q \beta} \rightarrow L_{q \beta}^{\prime \prime} \rightarrow 0$, car on a

$$
\begin{gathered}
0 \rightarrow L^{\prime} \otimes F_{q} V_{\beta} \mathscr{D}_{X} \rightarrow L \otimes F_{q} V_{\beta} \mathscr{D}_{X} \rightarrow L^{\prime \prime} \otimes F_{q} V_{\beta} \mathscr{D}_{X} \rightarrow 0 \\
\downarrow \\
\downarrow \\
\begin{array}{l}
\downarrow \\
0
\end{array} L^{\prime} \otimes \mathscr{D}_{X} \longrightarrow L \otimes \mathscr{D}_{X} \longrightarrow L^{\prime \prime} \otimes \mathscr{D}_{X} \longrightarrow
\end{gathered}
$$

Puisque $L_{q \alpha}^{\prime} \cap L_{p \beta}^{\prime}=L_{q \beta}^{\prime}$ (même pour $L^{\prime \prime}$ ) pour $\alpha>\beta, p>q$, on a $L_{q \alpha} \cap L_{p \beta}=L_{q \beta}$, cf. 1.1.9, donc $F_{q} V_{\beta} M=L_{q \beta}$ en prenant la limite. L'autre assertion est claire. 
3.3.8. Definition. On dit que $(M, F, V) \in M F V\left(\mathscr{D}_{X}\right)$ est un Module induit, si $(M, F, V) \simeq \bigoplus_{p, \alpha} L_{p \alpha} \otimes\left(\mathscr{D}_{X}, F[p], V[\alpha]\right)$ pour des $\mathcal{O}_{X}$-Modules $L_{p, \alpha}$ satisfaisant les condition de 3.3.6 $(-1 \leqslant \alpha \leqslant 0)$. Soit $K F V_{i}\left(\mathscr{D}_{X}\right)$ la sous-catégorie pleine de $K F V\left(\mathscr{D}_{X}\right)$ des Modules induits, posons

$$
D^{*} F V_{i}\left(\mathscr{D}_{X}\right)=K^{*} F V_{i}\left(\mathscr{D}_{X}\right) / K^{*} F V_{i}\left(\mathscr{D}_{X}\right)^{\emptyset}
$$

pour $*=\emptyset,+,-, b$, on a alors l'équivalence de catégories

$$
D^{-} F V_{i}\left(\mathscr{D}_{X}\right) \stackrel{\sim}{\rightarrow} D^{-} F V\left(\mathscr{D}_{X}\right)
$$

car:

3.3.9. Lemme. Soit $(M, F, V) \in M F V\left(\mathscr{D}_{X}\right)$, soit $\Lambda$ un sous-ensemble fini de $\boldsymbol{Q} \cap[-1,0]$ tel que $G r_{\alpha}^{V} M=0$ si $\alpha \in(\boldsymbol{Q} \cap[-1,0]) \backslash \Lambda$; alors $F_{p} V_{\alpha} M$ satisfait les conditions de 3.3.6 pour $-1 \leqslant \alpha \leqslant 0$, de plus, le morphisme naturel

$$
u: \bigoplus_{p \in Z, \alpha \in \Lambda} F_{p} V_{\alpha} M \otimes\left(\mathscr{D}_{X}, F[p], V[\alpha]\right) \rightarrow(M, F, V)
$$

est un epimorphisme strict et $\operatorname{Ker} u \in M V F\left(\mathscr{D}_{X}\right)$.

Preuve. La première assertion résulte de $t: F_{p} V_{\alpha} M \underset{\rightarrow}{\rightarrow} F_{p} V_{\alpha-1} M(\alpha<0)$ et $\left(V_{0} M\right) t$ $\subset V_{-1} M$. Soit $u:(N, F, V) \rightarrow(M, F, V)$ un morphisme de $M F V\left(\mathscr{D}_{X}\right)$, alors $u$ est un épimorphisme strict, sii $u\left(F_{p} V_{\alpha} N\right)=F_{p} V_{\alpha} M$ pour tout $p, \alpha$ (i.e. les trois filtrations $(F, V, \operatorname{Ker} u)$ de $N$ sont compatibles, cf. $\S 1)$; alors $\operatorname{Ker} u \in M F V\left(\mathscr{D}_{X}\right)$ résulte de la suite exacte

$$
0 \rightarrow F_{p} V_{\alpha}(\operatorname{Ker} u) \rightarrow F_{p} V_{\alpha} N \rightarrow F_{p} V_{\alpha} M \rightarrow 0 ;
$$

d'où l'assertion.

3.3.10. Lemme. Soit $(M, F, V)=L \otimes\left(\mathscr{D}_{X}, F[p], V[\alpha]\right)$ un Module induit, soit $g^{0}$ le foncteur des sections discontinues, alors $g^{0}(L)$ satisfait les conditions de 3.3.6, de plus, on a

$$
\left(\mathscr{g}^{0} L\right) \otimes\left(\mathscr{D}_{X}, F[p], V[\alpha]\right) \simeq \mathscr{g}^{0}\left(L \otimes\left(\mathscr{D}_{X}, F[p], V[\alpha]\right)\right)
$$

ou $(\bar{M}, F, V)=g^{\circ}(M, F, V)$ est defini par:

$$
\begin{aligned}
& \bar{M}=\lim _{\overrightarrow{q \beta}} g^{0}\left(F_{p} V_{\beta} M\right), \\
& F_{q} \bar{M}=\lim _{\vec{\beta}} g^{0}\left(F_{q} V_{\beta} M\right), \\
& V_{\beta} \bar{M}=\lim _{\vec{q}} g^{0}\left(F_{q} V_{\beta} M\right) .
\end{aligned}
$$


Preuve. Soit (*) $0 \rightarrow L^{\prime} \rightarrow L \rightarrow L^{\prime \prime} \rightarrow 0$ une suite exacte de $\mathcal{O}_{X}$-Modules telle que $L^{\prime} t=0$ et $L^{\prime \prime}$ est $t$-torsion, alors $g^{0}(*)$ est exacte et satisfait la même condition que $\left(^{*}\right)$, donc $\left(\mathscr{g}^{0} L\right) \otimes\left(\mathscr{D}_{X}, F[p], V[\alpha]\right)$ est bien défini; alors l'assertion résulte de

$$
\begin{aligned}
\mathscr{g}^{0}\left(\operatorname{Im}\left(L \otimes F_{q} V_{\beta} \mathscr{D}_{X} \rightarrow L \otimes \mathscr{D}_{X}\right)\right. & \simeq \operatorname{Im}\left(\mathscr{g}^{0}\left(L \otimes F_{q} V_{\beta} \mathscr{D}_{X}\right) \rightarrow g^{0}\left(L \otimes \mathscr{D}_{X}\right)\right. \\
& \simeq \operatorname{Im}\left(\left(\mathscr{g}^{0} L\right) \otimes F_{q} V_{\beta} \mathscr{D}_{X} \rightarrow\left(g^{0} L\right) \otimes \mathscr{D}_{X}\right)
\end{aligned}
$$

car $F_{q} V_{\beta} \mathscr{D}_{X}$ est localement libre de type fini sur $\mathcal{O}_{X}$ et $\left(\mathscr{g}^{0} L\right) \otimes \mathscr{D}_{X} \rightarrow \mathscr{I}^{0}\left(L \otimes \mathscr{D}_{X}\right)$ est injectif.

3.3.11. Lemmc. Soit $\left(M_{i}, F, V\right)=L_{i} \otimes\left(\mathscr{D}_{X}, F[p], V[\alpha]\right)$ un Module induit, soit $0 \rightarrow L_{i}^{\prime} \rightarrow L_{i} \rightarrow L_{i}^{\prime \prime} \rightarrow 0$ une suite exacte telle que $L_{i}^{\prime} t=0$ et $L_{i}^{\prime \prime}$ est sans $t$ torsion $(i=1,2)$. Soit $u:\left(M_{1}, F, V\right) \rightarrow\left(M_{2}, F, V\right)$ un morphisme de $\operatorname{MFV}\left(\mathscr{D}_{X}\right)$ induit par $\bar{u}: L_{1} \rightarrow L_{2}$. Si $\bar{u}$ est injectif et $L_{2}^{\prime \prime} / \bar{u}\left(L_{1}^{\prime \prime}\right)$ est sans $t$-torsion, alors $u$ est un monomorphisme strict et Coker $u \simeq \operatorname{Coker} \bar{u} \otimes\left(\mathscr{D}_{X}, F[p], V[\alpha]\right)$.

Preuve. Si $L_{i} t=0(i=1,2)$, l'assertion est claire. Si $L_{i}$ sont sans $t$-torsion, l'assertion résulte de

$$
\begin{aligned}
& 0 \rightarrow L_{i} \otimes F_{q} V_{\beta} \mathscr{D}_{X} \rightarrow L_{2} \otimes F_{q} V_{\beta} \mathscr{D}_{X} \rightarrow L_{3} \otimes F_{q} V_{\beta} \mathscr{D}_{X} \rightarrow 0 \\
& 0 \rightarrow L_{1} \otimes \mathscr{D}_{X} \longrightarrow L_{2} \otimes \mathscr{D}_{X} \longrightarrow L_{3} \otimes \mathscr{D}_{X} \longrightarrow 0
\end{aligned}
$$

où $L_{3}=$ Coker $\bar{u}$. Dans le cas général, posons

$$
\left(M_{i}^{*}, F, V\right)=L_{i}^{*} \otimes\left(\mathscr{D}_{X}, F[p], V[\alpha]\right)
$$

pour $i=1,2,3, *=\emptyset,{ }^{\prime}$, ", où $L_{3}=$ Coker $\bar{u}$; alors

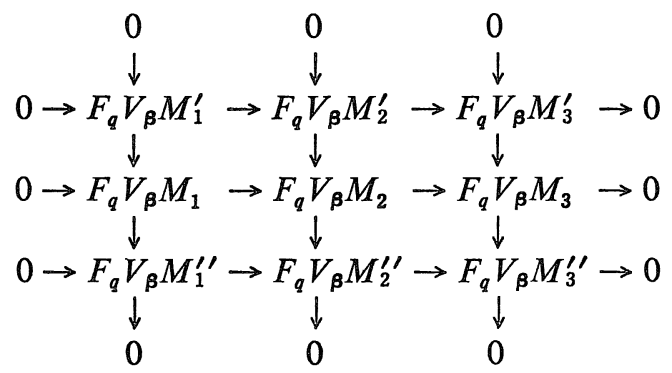

d'où l'assertion.

3.3.12. Définition. Soient $f: X \rightarrow Y$ et $t: Y \rightarrow S$ des morphismes de variétés complexes lisses tels que $t$ et $t f$ sont lisses. Supposons que $S$ est un disque ouvert, posons $Y_{0}=t^{-1}(0), X_{0}=f^{-1}\left(Y_{0}\right)$. Soit $\operatorname{MFV}\left(\mathscr{D}_{X}\right)$ comme dans 3.3.1. On définit 
$\operatorname{MFV}\left(f^{-1} \mathscr{D}_{Y}\right)$ et $M F V_{i}\left(f^{-1} \mathscr{D}_{Y}\right)$, etc. en remplacant $\mathscr{D}_{X}, \mathcal{O}_{X}$ par $f^{-1} \mathscr{D}_{Y}, f^{-1} \mathcal{O}_{Y}$.

Soit $\left(\mathscr{D}_{X \rightarrow Y}, F, V\right)=\mathcal{O}_{X} \otimes_{f^{-1} \mathcal{O}_{Y}} f^{-1}\left(\mathscr{D}_{Y}, F, V\right)$; on définit le foncteur $D R_{X / Y}$ : $M F V\left(\mathscr{D}_{X}\right) \rightarrow \operatorname{MFV}\left(f^{-1} \mathscr{D}_{Y}\right)$ par $\bigotimes_{\mathscr{D}_{X}} \mathscr{D}_{X \rightarrow Y}$, i.e. $(\bar{M}, F, V)=D R_{X / Y}(M, F, V)$ est défini par:

$$
\begin{aligned}
& \bar{M}=M \otimes_{\mathscr{D}_{X}} \mathscr{D}_{X \rightarrow Y} \\
& F_{p} \bar{M}=\operatorname{Im}\left(\bigoplus_{i+j=p} F_{i} M \otimes F_{j} \mathscr{D}_{X \rightarrow Y} \rightarrow \bar{M}\right) \\
& V_{\alpha} \bar{M}=\operatorname{Im}\left(\bigoplus_{\beta+\gamma=\alpha} V_{\beta} M \otimes V_{\gamma} \mathscr{D}_{X \rightarrow Y} \rightarrow \bar{M}\right)
\end{aligned}
$$
$V[\alpha])$.

3.3.13. Leemme. $D R_{X / Y}\left(L \bigotimes_{\mathcal{O}_{X}}\left(\mathscr{D}_{X}, F[p], V[\alpha]\right)\right) \simeq L \underset{f^{-1} \mathscr{O}_{Y}}{\bigotimes}\left(f^{-1} \mathscr{D}_{Y}, F[p]\right.$,

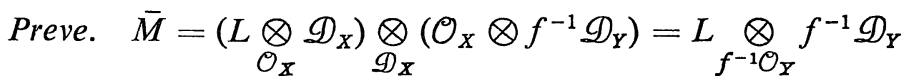

$$
\begin{aligned}
& F_{q} \bar{M}=\operatorname{Im}\left(\bigoplus_{i+j=q} L \otimes F_{i-p} \mathscr{D}_{X} \otimes F_{j} \mathscr{D}_{X \rightarrow Y} \rightarrow L \bigotimes_{O_{X}} \mathscr{D}_{X \rightarrow Y}\right) \\
& =\operatorname{Im}\left(L \otimes F_{q-p} \mathscr{D}_{X \rightarrow Y} \rightarrow L \otimes_{\mathcal{O}_{X}} \mathscr{D}_{X \rightarrow Y}\right) \\
& =\operatorname{Im}\left(L \otimes f^{-1} F_{q-p} \mathscr{D}_{Y} \rightarrow L \otimes_{f^{-1} \Theta_{Y}} f^{-1} \mathscr{D}_{Y}\right)
\end{aligned}
$$

(de même pour $V$ ) car $F_{p} \mathscr{D}_{X} F_{q} \mathscr{D}_{X \rightarrow Y} \subset F_{p+q} \mathscr{D}_{X \rightarrow Y}, V_{\alpha} \mathscr{D}_{X} V_{\beta} \mathscr{D}_{X \rightarrow Y} \subset V_{\alpha+\beta} \mathscr{D}_{X \rightarrow Y}$ et $1 \in F_{0} V_{0} \mathscr{D}_{X}$.

3.3.14. Lemme. $D R_{X / Y}\left(K F V_{i}\left(\mathscr{D}_{X}\right)^{\emptyset}\right) \subset K F V_{i}\left(f^{-1} \mathscr{D}_{Y}\right)^{\emptyset}$.

Preuve. Soient $Z=X \underset{S}{\times} Y, i: X \hookrightarrow Z, p: Z \rightarrow Y$. Puisque $D R_{X / Y}=i^{-1} D R_{Z / Y}$ $D R_{X / Z}$, on peut supposer que $f$ est une immersion fermée ou lisse.

Si $f$ est une immersion fermée, $D R_{X / Y}$ est localement donné par $\underset{C}{\bigotimes_{C}}\left[\partial^{\prime}\right]$ donc l'assertion est claire. Si $f$ est lisse, on a localement $X=W \times Y, f=p r_{2}$. On définit $\widetilde{D R}_{W} M=M \otimes \Lambda^{-\bullet} \Theta_{W}$, i.e. le complexe de Koszul pour $\partial_{1}, \cdots, \partial_{n}$, où $\left(x_{1}, \cdots, x_{n}\right)$ sont des coordonnées de $W$. On définit les filtrations par

$$
\begin{aligned}
& F_{p}\left(M \otimes \Lambda^{-i} \Theta_{W}\right)=F_{p+i} M \otimes \Lambda^{-i} \Theta_{W}, \\
& V_{\alpha}\left(M \otimes \Lambda^{-i} \Theta_{W}\right)=V_{\alpha} M \otimes \Lambda^{-i} \Theta_{W},
\end{aligned}
$$

alors le morphisme $M \rightarrow M \otimes \mathscr{D}_{X \rightarrow Y}\left(u \mapsto u \otimes 1_{X \rightarrow Y}\right)$ induit un isomorphisme

$$
\widetilde{D R}_{W}(M, F, V) \stackrel{\sim}{\rightarrow} D R_{X / Y}(M, F, V) ;
$$

en effet on peut supposer que $L$ est sans $t$-torsion où $L t=0$, alors on peut réduire au cas $L=\mathcal{O}_{X}$ ou $\mathcal{O}_{X_{0}}$, et vérifier (3.3.14.1) directement.

Si $\left(M^{*}, F, V\right) \in K F V_{i}\left(\mathscr{D}_{X}\right)^{\emptyset}$, chaque $F_{p} V_{\alpha} \widetilde{D R_{W}} M^{*}$ est acyclique, d'où l'assertion. 
3.3.15. Supposons que $f: X \rightarrow Y$ est propre. On définit

$$
f_{*}: D^{*} F V_{i}\left(\mathscr{D}_{X}\right) \rightarrow D^{*} F V_{i}\left(\mathscr{D}_{Y}\right)
$$

pour $*=\emptyset,+,-, b, \operatorname{par} f_{*}=f_{*} D R_{X / Y}$, où

$$
f_{*}: D^{*} F V_{i}\left(f^{-1} \mathscr{D}_{X}\right) \rightarrow D^{*} F V_{i}\left(\mathscr{D}_{Y}\right)
$$

est le foncteur image directe, qui est bien défini d'après 3.3.10 et 3.3.11. On notera encore $f_{*}$ la composition

$$
D^{-} F V\left(\mathscr{D}_{X}\right) \underset{\leftarrow}{\leftarrow} D^{-} F V_{i}\left(\mathscr{D}_{X}\right) \stackrel{f_{*}}{\rightarrow} D^{-} F V_{i}\left(\mathscr{D}_{X}\right) \stackrel{\sim}{\rightarrow} D^{-} F V\left(\mathscr{D}_{Y}\right) .
$$

Notons que, par définition, le foncteur $G r_{a}^{V}$ commute au foncteur image direct, i.e. $f_{0 *} G r_{a}^{V}=G r_{\alpha}^{V} f_{*}$, où $f_{0}: X_{0} \rightarrow Y_{0}$.

3.3.16. Lemme. Soit $(M, F, V) \in M F V\left(\mathscr{D}_{X}\right)$. Si chaque $F_{p} V_{\alpha} M$ est coherent $\operatorname{sur} \mathcal{O}_{X}$, on $a f_{*}(M, F, V) \in D^{-} F V_{c}\left(\mathscr{D}_{Y}\right)$. Si l'action de $t \partial_{t}-\alpha$ sur $G r_{\alpha}^{V} M$ est nilpotente, on a $f_{*}(M, F, V) \in D^{-} F V_{m}\left(\mathscr{D}_{Y}\right)$.

Preuve. Soit $\left(L^{\circ}, F, V\right) \underset{\rightarrow}{\sim}(M, F, V)$ la résolution canonique des Modules induits dans 3.3.9. Si $F_{p} V_{\alpha} M$ sont cohérents, alors $F_{p} V_{\alpha} L^{i}$ sont cohérents, donc $\mathscr{H}^{i}\left(F_{p} V_{\alpha} D R_{X / Y} L^{\circ}\right)$ et $\mathscr{H}^{i}\left(\mathbb{R} f_{*} F_{p} V_{\alpha} D R_{X / Y} L^{\circ}\right)$ sont cohérents sur $\mathcal{O}_{X}$ et $\mathcal{O}_{Y}$.

Si $\left(t \partial_{t}-\alpha\right)^{m}: G r_{a}^{V} M \rightarrow G r_{a}^{V} M$ est nul, $\left(t \partial_{t}-\alpha\right)^{m}:\left(G r_{a}^{V} L^{0}, F\right) \rightarrow\left(G r_{a}^{V} L^{0}, F\right)$ est nul dans $D F\left(\mathscr{D}_{X_{0}}\right)$ donc l'action de $\left(t \partial_{t}-\alpha\right)^{m}$ sur $\mathscr{H}^{i} f_{0 *} G r_{\alpha}^{V} L \simeq \mathcal{H}^{i} G r_{\alpha}^{V} f_{*} L^{\bullet}$ est nulle; d'où l'assertion.

3.3.17. Proposition. Soit $f: X \rightarrow Y$ comme dans 3.3.15, posons $f_{0}=\left.f\right|_{X_{0}}: X_{0}$ $\rightarrow Y_{0}$. Soit $(M, F)$ un $\mathscr{D}_{X}$-Module filtré cohérent, quasi-unipotent et regulier le long de $X_{0}$. Supposons que $f_{0 *} G r_{\alpha}^{V}(M, F)$ soit strict pour tout $\alpha \in Q$, alors $f_{*}(M, F, V)$ est strict dans un voisinage de $Y_{0}$, la filtration induite de $\mathcal{H}^{i} f_{*}(M, F)$ par $V$ est la filtration de Malgrange-Kashiwara, $\mathcal{H}^{i} f_{*}(M, F)$ est quasi-unipotent et regulier le long de $Y_{0}$, et $G_{a}^{V} \mathcal{H}^{i} f_{*}(M, F) \simeq \mathcal{H}^{i} f_{*} G r_{a}^{V}(M, F)$.

Preuve. Puisque $f$ est propre, et $G r_{\alpha}^{V}(M, F),(M, F)$ sont cohérents, il existe un épimorphisme strict localement (rel. à $Y$ ):

$$
\bigotimes_{p, \alpha} F_{p} V_{\infty} \otimes\left(\mathscr{D}_{X}, F[p], V[\alpha]\right) \rightarrow(M, F, V)
$$

où la somme directe est étendue sur un nombre fini de $p, \alpha$ (d'après le lemme de Nakayama). Par le même argument que dans 3.3.9, on obtient localement (relatif à $Y)$ une résolution de $(M, F, V)$ par des Modules induits cohérents, donc on voit que $\mathscr{H}^{i} V_{\alpha} f_{*} M$ (resp. $\mathscr{H}^{i} f_{*}(M, F) \in M G\left(\mathscr{B}_{Y}\right)$ ) sont cohérents sur $V_{0} \mathscr{D}_{Y}$ (resp. $\mathscr{B}_{Y}$ ), donc l'assertion résulte de $3.3 .3,3.3 .5$ et de 3.3 .16 , car $V_{\alpha} \mathcal{H}^{i} f_{*} M=$ 
$\mathscr{H}^{i} f_{*} M$ sur $Y \backslash Y_{0}$

3.4. Cycles evanescents (cf. [17], [22])

3.4.1. Soit $p: X \rightarrow S$ un morphisme lisse sur un disque ouvert $S$. Soient $X_{0}=$ $p^{-1}(0), S_{0}=\{0\}$, et $t$ la coordonnée de $S(\subset C)$. On définit $M V\left(p^{-1} \mathscr{D}_{S}\right)$ comme dans 3.3.1, i.e. $(M, V) \in M V\left(p^{-1} \mathscr{D}_{S}\right)$ sii:

$$
\begin{aligned}
& \bigcup_{\alpha \in Q} V_{\alpha} M=M, \\
& \left(V_{\alpha} M\right)\left(V_{\beta} p^{-1} \mathscr{D}_{S}\right) \subset V_{\alpha+\beta} M, \\
& t: V_{\alpha} M \stackrel{\sim}{\rightarrow} V_{\alpha-1} M \quad(\alpha<0) .
\end{aligned}
$$

Soit $D V\left(p^{-1} \mathscr{D}_{S}\right)$ la catégorie derivée correspondante. On définit $D V_{m}\left(p^{-1} \mathscr{D}_{S}\right)$ comme dans 3.3.1, et $D V_{c}\left(p^{-1} \mathscr{D}_{S}\right)$ par:

$\left(M^{*}, V\right) \in D V_{c}\left(p^{-1} \mathscr{D}_{S}\right)$ sii $\mathscr{H}^{i} V_{\alpha} M_{x}^{*}$ est de type fini sur $\mathcal{O}_{s, 0}$ pour tout $x \in X_{0}$.

3.4.2. Lemme. Si $\left(M^{*}, V\right) \in D^{-} V_{m, c}\left(p^{-1} \mathscr{D}_{S}\right)$, alors $\left(M^{*}, V\right)$ est strict et $\mathscr{H}^{i} V_{\alpha} M_{x}$ est libre sur $\mathcal{O}_{S, 0}$ pour $x \in X_{0}, \alpha<0$.

(Même démonstration que 3.3.2 et 3.3.3.)

3.4.3. Soient $S^{*}=S \backslash\{0\}$, et $\pi: \tilde{S}^{*} \rightarrow S^{*}$ un revêtement universel; posons

$$
\tilde{X}^{*}=X \underset{S}{X} \tilde{S}^{*}, \quad j: \tilde{X}^{*} \rightarrow X, \quad i: X_{0} \rightarrow X .
$$

On définit les foncteurs $\psi$ et $\phi$ de $D(A)$ dans $D\left(\left.A\right|_{x_{0}}\right)$ pour $A=\boldsymbol{C}_{X}, p^{-1} \mathscr{D}_{S}$, etc., par:

$$
\begin{aligned}
& \psi L^{\cdot}=i^{-1} R j_{*} j^{-1} L^{\cdot} \\
& \phi L^{\cdot}=\operatorname{Cone}\left(i^{-1} L^{\cdot}[-1] \rightarrow i^{-1} R j_{*} j^{-1} L^{\cdot}[-1]\right)[1], \text { cf. [10]. }
\end{aligned}
$$

Posons $p_{0}: X_{0} \rightarrow S_{0}(=\{0\}), \bar{j}: \tilde{S}^{*} \rightarrow S$ et

$$
\tilde{\mathcal{O}}^{\alpha}=t^{\alpha} \boldsymbol{C}\{t\}[\log t] \subset\left(\bar{j}_{*} \Theta_{\left.\widetilde{s}^{*}\right)_{0}} .\right.
$$

Soit $\alpha<0$, on définit le foncteur $\psi_{a}^{a l}: D V\left(p^{-1} \mathscr{D}_{S}\right) \rightarrow D V\left(p_{0}^{-1} \mathscr{D}_{S, 0}\right)$ par:

$$
\begin{gathered}
\psi_{\alpha}^{a l} M^{\bullet}=i^{-1} V_{\alpha} M^{\bullet} \bigotimes_{C\left\{t^{t}\right]} \tilde{\mathcal{O}}^{\alpha}\left[t^{-1}\right] \\
V_{\beta}\left(\psi_{\alpha}^{a l} M^{*}\right)=i^{-1} V_{\alpha+\beta+[-\beta]} M^{\bullet} \bigotimes_{C\left\{{ }^{t}\right\}} \tilde{\mathcal{O}}^{\alpha+[-\beta]}
\end{gathered}
$$

(notons que $V_{\alpha} M^{j}$ sont sans $t$-torsion pour $\alpha<0$, et que $\tilde{\mathcal{O}}^{\alpha}\left[t^{-1}\right]$ est un $\mathscr{D}_{S, 0^{-}}$ module à gauche); on a alors un iosmorphisme

$$
\psi_{a-1}^{a l}\left(M^{*}, V\right) \simeq \psi_{a}^{a l}\left(M^{*}, V\right) \text { si } \quad \alpha<0
$$


et un morphisme naturel $\psi_{a}^{a l} M^{*} \rightarrow \psi M^{*}$.

On définit $\widetilde{D R}_{S} \psi_{\infty}^{a l}\left(M^{*}, V\right) \in D V\left(C_{X_{0}}\right)$ par

$$
\text { Cone }\left(\partial_{t}: \psi_{a}^{a l} M^{*} \rightarrow \psi_{a}^{a j} M^{*}\right)
$$

avec la filtration $V_{\beta}=\operatorname{Cone}\left(\partial_{t}: V_{\beta-1} \psi_{a}^{a l} M^{*} \rightarrow V_{\beta} \psi_{a}^{a k} M^{*}\right)$.

Soient $\left(M^{\circ}, V\right) \in D V\left(p^{-1} \mathscr{D}_{S}\right)$ et $\alpha<0$, on définit

$$
A_{\infty}: V_{0} \widetilde{D R}_{S} \psi_{a}^{a l} M^{*} \rightarrow G r_{a}^{V} M^{*}[1]
$$

par Cone $(p r, 0):$ Cone $\left(\partial_{t}: V_{-1} \psi_{a}^{a l} M^{*} \rightarrow V_{0} \psi_{a}^{a l} M^{\bullet}\right) \rightarrow$ Cone $\left(G r_{a}^{V} M^{*} \rightarrow 0\right)$, où $p r$ : $V_{-1} \psi_{a}^{a l} M^{\bullet} \rightarrow G r_{a}^{V} M$ est définie par:

$$
\sum u_{j} \otimes t^{\alpha+1}(\log t)^{j} / j ! \mapsto u_{0} \quad \text { pour } \quad u_{j} \in i^{-1} V_{\alpha} M .
$$

On définit $B_{a}: V_{0} \widetilde{D R}_{S} \psi_{a}^{a l} M^{\circ} \rightarrow \widetilde{D R}_{S} \psi_{a}^{a l} M^{\circ} \rightarrow \widetilde{D R}_{S} \psi M^{*}$ par la composition des morphismes naturels.

3.4.4. Lemme. $S i\left(M^{*}, V\right) \in D^{-} V_{m, c}\left(p^{-1} \mathscr{D}_{S}\right), A_{\alpha}$ est un isomorphisme.

Preuve. En utilisant la filtration canonique sur $M^{\circ}$, on peut supposer que $(M, V) \in M V_{m, c}\left(p^{-1} \mathscr{D}_{s}\right), \operatorname{car}(M, V)$ est strict et le foncteur $\otimes_{C^{(t)}} \tilde{\mathcal{O}}^{\alpha}$ est exact. Par définition, l'action de $\partial_{t}$ sur $\psi_{a}^{a l} M$ s'écrit

$$
\begin{aligned}
& \left(u \otimes t^{\alpha+1}(\log t)^{j} / j !\right) \partial_{t} \\
& \quad=u\left(t \partial_{t}-\alpha\right) \otimes t^{\alpha}(\log t)^{j} / j !-u \otimes t^{\alpha}(\log t)^{j-1} /(j-1) !
\end{aligned}
$$

si $u \in i^{-1} V_{\alpha} M$. Soit $W$ la filtration de $\psi_{\alpha}^{a l} M$ par degré de log $t$. Puisque $V_{\beta} M_{x}$ est un $\boldsymbol{C}\{t\}$-module libre de type fini pour $x \in X_{0}(\beta<0)$, et l'action de $t \partial_{t}-\alpha$ sur $G r_{\beta}^{V} M$ est bijectif si $\alpha \neq \beta, t \partial_{t}-\alpha: V_{\beta} M_{x} \rightarrow V_{\beta} M_{x}$ est bijectif si $\alpha>\beta$; donc $G r_{i}^{W} V_{<0} \tilde{D R_{S}} \psi_{a}^{a l} M$ et $V_{<0} \widetilde{D R}_{S} \psi_{a}^{a l} M$ sont acycliques $(\alpha<0)$. Puisque $V_{<-1} \psi_{a}^{a l}$ $M=i^{-1} V_{<\infty} M^{*} \otimes \tilde{\mathcal{O}}^{\alpha+1}$ et $\operatorname{pr}\left(V_{<-1} \psi_{\alpha}^{a l} M\right)=0$, il reste à démontrer que

$$
(p r, 0): G r_{0}^{V} D R_{S} \psi_{a}^{a l}(M, V) \rightarrow G r_{a}^{V} M[1]
$$

est un isomorphisme. On peut supposer que $\left(G r_{\alpha}^{V} M\right)\left(t \partial_{t}-\alpha\right)=0$ en utilisant une filtration de $G r_{\alpha}^{V} M$, car $t \partial_{t}-\alpha$ est nilpotente sur $G r_{a}^{V} M$; on a alors

$$
\left(W_{i} G r_{-1}^{V} \psi_{a}^{a l} M\right) \partial_{t} \subset W_{i-1} G r_{0}^{V} \psi_{a}^{a l} M
$$

et

$$
\partial_{t}: G r_{i}^{W} G r_{-1}^{V} \psi_{a}^{a l} M \stackrel{G}{\rightarrow} G r_{W}^{i-1} G r_{0}^{V} \psi_{a}^{a l} M \quad(i \geqslant 1),
$$

$\operatorname{car} G r_{-j}^{V} \psi_{\infty}^{a l} M=i^{-1} G r_{\infty}^{V} M \bigotimes_{C} t^{\alpha+j} C[\log t]$ pour $j=0,1$; d'où l'assertion. 
3.4.5. Lemme. Soient $x_{0} \in X_{0}$ et $B_{\mathrm{e}}=\left\{x \in X \mid\left\|x-x_{0}\right\|<\varepsilon\right\}$, où on choisit des coordonnées de X pour définir la norme \|\| . Posons $p_{\varepsilon}=\left.p\right|_{B_{\varepsilon^{*}}} \quad$ Soit $\left(M^{*}, V\right)$ $\in D^{-} V_{m}\left(p^{-1} \mathscr{D}_{S}\right)$. Si $R^{i} p_{\boldsymbol{\varepsilon}_{*}} V_{\alpha} M^{*}$ est cohérent sur $\mathcal{O}_{S}$ dans un voisinage de 0 pour $1 \gg \varepsilon>0$, alors

$$
\bigoplus_{-1 \leqslant \infty<0} B_{\alpha}: \bigoplus_{-1<\infty<0}\left(V_{0} \widetilde{D R}_{S} \psi_{\alpha}^{a l} M^{*}\right)_{x_{0}} \rightarrow\left(\widetilde{D R}_{S} \psi M^{*}\right)_{x_{0}}
$$

et est un isomorphisme.

Preuve. Puisque l'action de $t \partial_{t}-\alpha$ sur $R p_{\mathbf{z}^{*}} G r_{\alpha}^{V} M^{*}$ est nilpotente, $R p_{\mathbf{z}^{*}}\left(\left(V_{\alpha} / V_{\beta}\right)\right.$ $M, V)$ est strict, donc $R^{i} p_{\mathrm{e}^{*}} V_{\alpha} M^{*}$ est libre sur $\mathcal{O}_{S}$ dans un voisinage de $0(\alpha<0)$ et $R p_{z^{*}}\left(M^{*}, V\right)$ est strict par le même argument que 3.3.2, 3.3.3 et 3.4.2 (notons que $\left.R^{i} p_{\mathrm{e}^{*}} V_{\alpha} M^{*}\right|_{s^{*}}$ ne dépend pas de $\alpha$.)

Si $p=i d$ (i.e. $X=S),(M, V) \in M V_{m}\left(\mathscr{D}_{S}\right)$ et $V_{\alpha} M$ est cohérent sur $\mathcal{O}_{S}$, alors $\oplus B_{\alpha}$ est un isomorphisme par la théorie classique des équations différentielles d'une variable à singularités régulières; en particulier, $H^{i}\left(V_{0} \widetilde{D R}_{S} \psi_{a}^{a l} M\right)=H^{i}$ $\left(\widetilde{D R}_{S} \psi M\right)=0$ si $i \neq-1$. On a donc

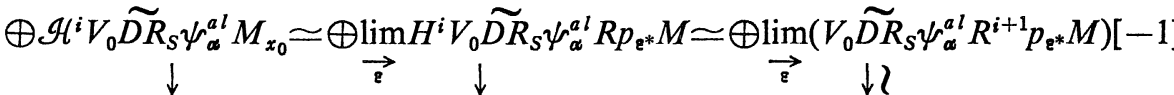

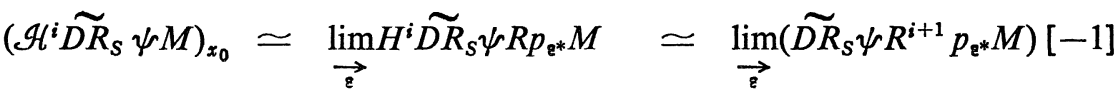

d'où l'assertion.

3.4.6. Lemme. Soit $(M, F)$ un $\mathscr{D}_{X}$-Module cohérent, quasi-unipotent et régulier le long de $X_{0}$ (cf. 3.2), alors $G r^{F} V_{\alpha} M$ est cohérent sur $G r^{F} V_{0} \mathscr{D}_{X}$ pour tout $\alpha \in \boldsymbol{Q}$.

Preuve. On peut supposer que $\alpha<0$, car $G r^{F} G r_{a}^{V} M$ sont cohérents sur $G r^{F} \mathscr{D}_{X_{0}}$ et $G r^{F} G r_{0}^{V} \mathscr{D}_{X} \simeq G r^{F} \mathcal{D}_{X_{0}}\left[t \partial_{t}\right]$. Puisque chaque $G r_{p}^{F} V_{\alpha} M$ est cohérent sur $\mathcal{O}_{X}$, il suffit de montrer que $G r^{F} V_{\alpha} M$ est localement de type fini sur $G r^{F} V_{0} \mathscr{D}_{X}$, i.e. il existe localement $p \in \boldsymbol{Z}$ tel que

$$
\left(G r_{p}^{F} V_{\alpha} M\right)\left(G r_{i}^{F} V_{0} \mathscr{D}_{X}\right)=G r_{p+i}^{F} V_{\alpha} M \text { pour tout } i \geqslant 0 \text {. }
$$

Puisque $V_{\alpha} M=M$ et $V_{0} \mathscr{D}_{X}=\mathscr{D}_{X}$ sur $X^{*}$, on a localement (rel. à $X$ ) $p_{1} \in Z$ tel qu'on ait (3.4.6.1) sur $X^{*}$. D'autre part, $G r^{F} G r_{a}^{V} M \otimes_{\mathcal{O}_{X}} \mathcal{O}_{X_{0}}$ est cohérent sur $G r^{F} \mathscr{D}_{X_{0}}$, donc on trouve aussi localement $p_{2} \in \boldsymbol{Z}$ tel qu'on ait (3.4.6.1) sur $X_{0}$, par le lemme de Nakayama. Posons $p=\max \left\{p_{1}, p_{2}\right\}$, alors l'assertion est claire.

3.4.7. Corollaire. Soient $p: X \rightarrow S(\subset C)$ un morphisme lisse et $X_{0}=p^{-1}(0)$. 
Soit $\mathscr{D}_{X / S}=\left\{P \in \mathscr{D}_{X}:[P, t]=0\right\}$ où $t$ est la coordonnée de $S$. Soit $(M, F)$ comme dans 3.4.6, alors $G r^{F} V_{\alpha} M$ est cohérent sur $G r^{F} \mathscr{D}_{X / s}$ dans un voisinage de $X_{0}$.

Preuve. On peut supposer que $\alpha<0$, alors, pour tout $x \in X_{0}, G r^{F} V_{\alpha} M_{x}$ est de type fini sur $G r^{F} \mathscr{D}_{X / S, x}$ par le même argument que dans 3.4.6, car $G r^{F} \mathscr{D}_{X / S} \bigotimes_{O_{X}}$ $\mathcal{O}_{X_{0}} \simeq G r^{F} \mathscr{D}_{X_{0}}$; donc il existe un $G r^{F} \mathscr{D}_{X / S}$-Module gradué cohérent $N$ dans un voisinage de $x$ tel que $N_{x}=G r^{F} V_{\alpha} M_{x}$; de plus, on peut supposer que l'action de $t \partial_{t}$ sur $G r^{F} V_{\alpha} M_{x}$ est prolongée sur $N$, alors $N$ est un $G r^{F} V_{0} \mathscr{D}_{X}$-Module car $G r^{F} V_{0} \mathscr{D}_{X}=G r^{F} \mathscr{D}_{X / S} \bigotimes_{C} \mathbb{C}\left[t \partial_{t}\right]$, et $N$ est cohérent sur $G r^{F} V_{0} \mathscr{D}_{X}$, donc $N=$ $G r^{F} V_{\alpha} M$ dans un voisinage de $x$; d'où l'assertion. (Notons que la condition sur $(M, F)$ implique que $\operatorname{supp}\left(G r^{F} V_{\alpha} M\right)^{\sim} \subset$ Specan $G r^{F} V_{0} \mathscr{D}_{X}$ est fini sur Specan $G r^{F} \mathscr{D}_{X / S}$ dans un voisinage de (Specan $\left.G r^{F} V_{0} \mathscr{D}_{X}\right)\left.\right|_{X_{0}}$ )

3.4.8. Proposition. Soient $p: X \rightarrow S, X_{0}$ et $(M, F)$ comme dans 3.4.7, soit $V$ la filtration rationnelle de $M$. Si $M$ est holonome, alors $D R_{X / s}(M, V) \in D^{-} V_{m}$ $\left(\mathscr{D}_{X}\right)$ satisfait la condition de 3.4.5.

Preuve. D'après 3.4.7, $D R_{X / S} V_{\alpha} M \simeq V_{\alpha} M \stackrel{L}{\bigotimes} \mathcal{O}_{X}$ est localement représenté par un complexe borné supérieurement de $\mathscr{\mathcal { O }}_{X / S}$-Modules dont les différentielles sont $p^{-1} \mathcal{O}_{s}$-linéaires. Pour appliquer un résultat de Kiehl-Verdier [20] (voir aussi [15]), il suffit de montrer:

3.4.9. Lemme. Soient $B_{\varepsilon}$ et $p_{\mathrm{\varepsilon}}$ comme dans 3.4.5, alors il existe $\varepsilon_{0}>0$ tel que, pour tout $\left.\varepsilon_{1} \in\right] 0, \varepsilon_{0}\left[\right.$, il existe $\left.\varepsilon_{2} \in\right] 0, \varepsilon_{1}[$ et $\eta>0$ tels que

$$
\left.R^{i} p_{\mathbf{z}^{*}} D R_{X / S} V_{\infty} M\right|_{s_{\eta}} \text { ne dépend pas de } \varepsilon \in\left[\varepsilon_{2}, \varepsilon_{1}\right] \text {, }
$$

où $S_{\eta}=\{|t|<\eta\}$.

Preuve. D'après $[19,4.4 .1$ et la preuve de 8.6 .2$]$ il suffit de montrer que

$$
S S\left(D R_{X / S} V_{\alpha} M\right) \subset \underset{\lambda}{\cup}\left(T_{X_{\lambda}}^{*} X+X \underset{S}{\times} T^{*} S\right)
$$

dans un voisinage de $x_{0}$ pour une stratification $\left\{X_{\lambda}\right\}$ satisfaisant la condition $A_{f}$ de Thom rel. à la stratification $S=S^{*} \cup\{0\}$. Notons que la condition $A_{f}$ implique que le terme à droite est fermé.

Soit $\left\{X_{\lambda}\right\}$ une stratification de $X$ telle que $\operatorname{Car}(M) \subset \bigcup_{\lambda} T_{X_{\lambda}}^{*} X$; d'après Hironaka [14], on peut supposer que $\left\{X_{\lambda}\right\}$ satisfait la condition $A_{f}$ au voisinage de $x_{0}$ 
Soit $T^{*}(X / S)=\operatorname{Coker}\left(X \times T_{S}^{*} S \hookrightarrow T^{*} X\right)\left(\simeq \operatorname{Specan} G r^{F} \mathscr{D}_{X / S}\right)$ et $\phi: T^{*} X \rightarrow$ $T^{*}(X / S)$ la projection, cf. [15]. D'après $[19,10.1 .5]$, on a

$$
S S\left(D R_{X / S} V_{\alpha} M\right)=\operatorname{Car}\left(V_{\alpha} M \mathscr{D}_{X / S} \mathscr{D}_{X}\right)=\phi^{-1} \operatorname{Car} \mathscr{D}_{\boldsymbol{x} / S}\left(V_{\alpha} M\right)
$$

où $\operatorname{Car} \mathscr{D}_{X / S}\left(V_{\alpha} M\right)=\operatorname{supp}\left(G r^{F} V_{\alpha} M\right)^{\sim} \subset T^{*}(X / S)$. Si $\alpha<0, G r^{F} V_{\alpha} M$ est sans $t$ torsion, donc

$$
\operatorname{Car} \mathscr{D}_{X / S}\left(V_{\alpha} M\right)=\overline{\operatorname{Car} \mathscr{D}_{X^{*} / S^{*}}\left(\left.M\right|_{X^{*}}\right)},\left.\quad \operatorname{car} V_{\alpha} M\right|_{X^{*}}=\left.M\right|_{X^{*}} ;
$$

de plus, $\left.\operatorname{Car} \mathscr{D}_{X / S}\left(V_{\alpha} M\right)\right|_{x_{0}}=\operatorname{Car}\left(V_{\alpha} M / V_{\alpha-1} M\right)=\bigcup_{\beta \leqslant \infty} \operatorname{Car}\left(G r_{\beta}^{V} M\right)\left(\subset T^{*} X_{0}\right)$. Puisque $\operatorname{Car} \mathscr{D}_{X / S}(M)=\phi \operatorname{Car}(M)$ si $\operatorname{Car}(M)$ est finie sur $T^{*}(X / S)$, on obtient l'assertion pour $\alpha<0$. Si $\alpha \geqslant 0$ on peut supposer que $\partial_{t}: G r_{-1}^{V} M \rightarrow G r_{0}^{V} M$ est surjectif, cf. 3.1.8, car, si supp $M \subset X_{0}$, i.e. $M=i_{*} M_{0}$ avec $i: X_{0} \hookrightarrow X$, $\operatorname{Car} \mathscr{D}_{X / S}$ $\left(V_{\alpha} M\right)=\operatorname{Car} M_{0}(\alpha \geqslant 0)$; on a alors

$$
\operatorname{Car}\left(G r_{0}^{V} M\right) \subset \operatorname{Car} \mathscr{D}_{\boldsymbol{X} / S}\left(V_{<0} M\right)
$$

donc

$$
\operatorname{Car} \mathscr{D}_{X / S}\left(V_{\alpha} M\right)=\operatorname{Car} \mathscr{D}_{X / S}\left(V_{<0} M\right) \text { pour tout } \alpha ;
$$

d'où l'assertion. Ce qui achève la démonstration de 3.4.8.

Remarque. Cette démonstration montre que $\operatorname{Gr}_{a}^{V} M$ sont holonomes, si $(M, F)$ est un $\mathscr{D}_{X}$-Module holonome filtré, quasi-unipotent et régulier le long de $X_{0}$.

3.4.10. Soit $\left(M^{*}, F\right) \in D V\left(p^{-1} \mathscr{D}_{S}\right)$, posons

$$
\phi_{\alpha}^{a l}(M, V)=\psi_{\alpha}^{a l}(M, V) \text { pour }-1<\alpha<0 .
$$

On définit $\phi_{0}^{a l}$ par

$$
\phi_{0}^{a l}(M, V)=\text { Cône }\left(i^{-1}(M, V) \rightarrow \psi_{-1}^{a l}(M, V)\right)
$$

où $i^{-1} M \rightarrow \psi_{-1}^{a l} M$ est défini par

$$
u \mapsto-u t^{1-[-\alpha]} \otimes t^{[-\alpha]-1} \quad \text { pour } u \in i^{-1} V_{\alpha} M .
$$

On définit $\widetilde{D R}_{S} \phi_{0}^{a l}(M, V)$ comme dans 3.4 .3 , i.e.

$$
V_{\alpha} \widetilde{D R}_{S} \phi_{0}^{a l} M=\operatorname{Cône}\left(\partial_{t}: V_{\alpha-1} \phi_{0}^{a l} M \rightarrow V_{\alpha} \phi_{0}^{a l} M\right) \text {. }
$$

Posons $\psi^{a l}=\bigoplus_{-1 \leqslant a<0} \psi_{a}^{a l}, \phi^{a l}=\bigoplus_{-1<\alpha \leqslant 0} \phi_{a}^{a l}$, alors

$$
\phi^{a l}(M, V)=\text { Cône }\left(i^{-1}(M, V) \rightarrow \psi^{a l}(M, V)\right) .
$$

On a un morphisme naturel 


$$
\text { can: } \left.\psi^{a l} \rightarrow \phi^{a l} \quad \text { (resp. can: } \psi \rightarrow \phi\right)
$$

défini par

$$
(0, i d): \text { Cône }\left(0 \rightarrow \psi^{(a l)}(M, V)\right) \rightarrow \operatorname{Cône}\left(i^{-1}(M, V) \rightarrow \psi^{(a l)}(M, V)\right),
$$

alors les morphismes naturels $\psi^{a l} M \rightarrow \psi M$ et $\phi^{a l} M \rightarrow \phi M$ commutent avec can.

Soit $T$ la monodromie. On définit l'action de $T_{s}$ et de $T_{u}$ sur $\tilde{\mathcal{O}}^{a}$ par

$$
\begin{aligned}
& T_{s}\left(t^{\alpha}(\log t)^{j}\right)=\exp (2 \pi i \alpha) t^{\alpha}(\log t)^{j} \\
& T_{u}\left(t^{\alpha}(\log t)^{j}\right)=t^{\infty}(\log t+2 \pi i)^{j}
\end{aligned}
$$

et leur action sur $\psi^{a b}$ et $\phi^{a l}$ par

$$
T_{*}(m \otimes q)=m \otimes T_{*} q \quad \text { pour } \quad *=s, u, \quad m \in M, g \in \tilde{\mathcal{O}}^{\infty},
$$

alors on obtient la décomposition de Jordan $T=T_{s} T_{u}$ sur $\psi^{a l}$ et $\phi^{a l}$.

Posons $N=\left(\log T_{u}\right) / 2 \pi i$, alors $N(\log t)^{j}=j(\log t)^{j-1}$. On définit

Var: $\phi_{0}^{a l} \rightarrow \psi_{-1}^{a l}$ par

$$
(0, N) \text { : Cône }\left(i^{-1}(M, V) \rightarrow \psi_{-1}^{a l}(M, V)\right) \rightarrow \text { Cône }\left(0 \rightarrow \psi_{-1}^{a l}(M, V)\right)
$$

et var: $\psi^{a l} M \rightarrow \phi^{a l} M$ (resp. var: $\psi M \rightarrow \phi M$ ) par

$$
(0, T-i d): \text { Cône }\left(i^{-1} M \rightarrow \psi^{(a l)} M\right) \rightarrow \text { Cône }\left(0 \rightarrow \psi^{(a l)} M\right),
$$

alors $\operatorname{Var} \circ \operatorname{can}=N$, $\operatorname{can} \circ \operatorname{Var}=N$, $\operatorname{can} \circ \operatorname{var}=T-i d$, varocan $=T-i d$.

Soit $B_{0}: V_{0} \widetilde{D R}_{S} \phi_{0}^{a l} M \rightarrow \widetilde{D R_{S}} \phi M$ le morphisme naturel; alors $\bigoplus_{-1<\alpha \leqslant 0} B_{\alpha}$ : $V_{0} \widetilde{D R}_{S} \phi^{a l} M \rightarrow \widetilde{D R_{S}} \phi M$ est un isomorphisme, si $\bigoplus_{-1 \leqslant a<0} B_{\alpha}: V_{0} \widetilde{D R}_{S} \psi^{a l} M \rightarrow \widetilde{D R_{S}} \psi M$ l'est et si $\left.(M, V) \in D^{-} V_{m, c} p^{-1} \mathscr{D}_{S}\right)\left(\right.$ car $\partial_{t}: G r_{\alpha-1}^{V} M \rightarrow G r_{\alpha}^{V} M$ est un isomorphisme si $\alpha>0$ ), de plus, $\oplus B_{\alpha}$ commute avec can, var et l'action de $T$.

On définit le morphisme $A_{0}: V_{0} \widetilde{D R}_{S} \phi_{0}^{a l} M \rightarrow G r_{0}^{V} M[1]$ par

$$
\begin{aligned}
& \text { Cône }\left[\begin{array}{cc}
i^{-1} V_{0} M \rightarrow V_{0} \psi_{-1}^{a l} M \\
\partial_{t} \uparrow & \partial_{t} \uparrow \\
i^{-1} V_{-1} M \rightarrow V_{-1} \psi_{-1}^{a l} M
\end{array}\right] \stackrel{\left(p r, A_{-1}\right)}{\longrightarrow} \\
& \text { Cône }\left[\begin{array}{ll}
G r_{0}^{V} M \longrightarrow & 0 \\
\partial_{t}{ }_{G r_{-1}^{V} M \stackrel{-i d, 0}{\longrightarrow}}^{\longrightarrow} & \prod_{-1}^{V} M
\end{array}\right] \stackrel{\left(\begin{array}{r}
-i d \\
0,0
\end{array}\right)}{\leftarrow} G r_{0}^{V} M[1]
\end{aligned}
$$

où $p r: i^{-1} V_{\alpha} M \rightarrow G r_{\alpha}^{V} M(\alpha=-1,0)$. Ici 


$$
\text { Cône }\left[\begin{array}{cc}
K^{-1,0} & \stackrel{\alpha}{\rightarrow} K^{0,0} \\
\beta^{\prime \uparrow} & \underset{K^{-1,-1}}{\alpha} \underset{\alpha^{\prime}}{\rightarrow} K^{0,-1}
\end{array}\right]
$$

est le complexe simple associé au complexe double

$$
\left[K^{-1,-1} \stackrel{\left(\alpha^{\prime},-\beta^{\prime}\right)}{\longrightarrow} K^{0,-1} \oplus K^{-1,0} \stackrel{\alpha+\beta}{\longrightarrow} K^{0,0}\right] .
$$

3.4.11. Lemme. $A_{0}$ est un isomorphisme, si $(M, V) \in D^{-} V_{m, c}\left(p^{-1} \mathscr{D}_{S}\right)$. On a

$$
\begin{aligned}
& A_{\infty} N=\left(t \partial_{t}-\alpha\right) A \text { pour }-1 \leqslant \alpha \leqslant 0, \\
& A_{0} \text { can }=\partial_{t} A_{-1}, \quad A_{-1} \text { Var }=t A_{0} \quad \text { dans } D\left(C_{X_{0}}\right) .
\end{aligned}
$$

Preuve. La première assertion résulte de l'isomorphisme

$$
\partial_{t}: i^{-1} V_{<-1} M \underset{\rightarrow}{\rightarrow} i^{-1} V_{<0} M \text { pour } M \in M V_{m, c}\left(p^{-1} \mathscr{D}_{S}\right)
$$

démontré dans la preuve de 3.4.4. Pour $-1 \leqslant \alpha<0$, on définit

$$
h: V_{0} \widetilde{D R}_{S} \psi_{\alpha}^{a l} M \rightarrow G r_{\alpha}^{V} M
$$

par $(0, p r):$ Cône $\left(V_{-1} \psi_{\alpha}^{a l} M \rightarrow V_{0} \psi_{\alpha}^{a l} M\right) \rightarrow G r_{\alpha}^{V} M$, alors $d h+h d=\left(t \partial_{t}-\alpha\right) A_{\infty}-$ $A_{\alpha} N$ d'après (3.4.4.1), on a donc

$$
\left(t \partial_{t}-\alpha\right) A_{\alpha}=A_{\infty} N \text { dans } D\left(C_{X_{0}}\right) \text { pour }-1 \leqslant \alpha<0,
$$

où cette assertion pour $\alpha=0$ résulte des dernières assertions, car $N=$ can॰Var.

Par définition, on a la factorisation $A_{0}=A_{0}^{\prime \prime-1} A_{0}^{\prime}$, où $A_{0}^{\prime}=\left(p r, A_{-1}\right)$ et $A_{0}^{\prime \prime}=$ $\left(\begin{array}{r}-i d, 0 \\ 0,0\end{array}\right)$. On définit

$$
\operatorname{can}^{\prime}: G r_{-1}^{V} M[1] \rightarrow \text { Cône }\left[\begin{array}{ccc}
G r_{0}^{V} M & \longrightarrow & 0 \\
\partial_{t} \uparrow & \uparrow \\
G r_{-1}^{V} M \stackrel{-i d}{\longrightarrow} G r_{-1}^{V} M
\end{array}\right]
$$

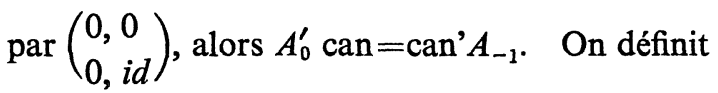

$$
h: G r_{-1}^{V} M[1] \rightarrow \text { Cône }\left[\begin{array}{cc}
G r_{0}^{V} M & 0 \\
\partial_{t} \uparrow & \\
G r_{-1}^{V} M \stackrel{-i d}{\longrightarrow} G r_{-1}^{V} M
\end{array}\right] \text { par }\left(\begin{array}{c}
0,0 \\
i d, 0
\end{array}\right),
$$

alors $d h+h d=A_{0}^{\prime \prime} \partial_{t}-$ can $^{\prime}$, donc $A_{0}$ can $=\partial_{t} A_{-1}$ dans $D\left(\boldsymbol{C}_{X_{0}}\right)$. 
On définit un sous-complexe de $G r_{0}^{V} \widetilde{D R}_{S} \phi_{0}^{a l} M$ par

$$
C=\text { Cône }\left[\begin{array}{c}
G r_{0}^{V} M \rightarrow G r_{0}^{V} \psi_{-1}^{a l} M \\
\prod_{0 \rightarrow\left(G r_{-1}^{V} \psi_{-1}^{a l} M\right)(\log t)}
\end{array}\right] .
$$

Soient $V_{0} \widetilde{D R}_{S} \phi_{0}^{a l} M \stackrel{a_{1}}{\rightarrow} G r_{0}^{V} \underset{D R_{S}}{{ }_{0}^{a l}} \stackrel{a_{2}}{\leftarrow} C \stackrel{a_{3}}{\rightarrow} G r_{0}^{V} M[1]$ les isomorphismes naturels, où $a_{3}=\left(\begin{array}{r}-i d, 0 \\ 0,0\end{array}\right)$; on a alors la factorisation $A_{0}^{\prime}=\bar{A}_{0}^{\prime} a_{1}$, et on vérifie $\bar{A}_{0}^{\prime} a_{2}$ $=A_{0}^{\prime \prime} a_{3}$; donc $a_{3} a_{2}^{-1} a_{1}=A_{0}$.

Soit $a_{1}^{\prime}: V_{0} \widetilde{D R}_{S} \psi_{-1}^{a l} M \rightarrow G r_{0}^{V} \widetilde{D R}_{S} \psi_{-1}^{a l} M$ l'isomorphisme naturel, on a alors la factorisation $A_{-1}=\bar{A}_{-1} a_{1}^{\prime}$ et Varo $a_{1}=a_{1}^{\prime} \circ \operatorname{Var}$. On définit $h: C \rightarrow G r_{-1}^{V} M$ par $\left(\begin{array}{c}0, p r \\ 0,0\end{array}\right)$, où

$$
p r: G r_{0}^{V} \psi_{-1}^{a l} M=G r_{-1}^{V} M \otimes t^{-1} C[\log t] \rightarrow G r_{-1}^{V} M
$$

est définie par $\operatorname{pr}\left(\sum u_{j} \otimes t^{-1}(\log t)^{j}\right)=u_{0}$; alors $h d+d h=t a_{3}-\bar{A}_{-1} \circ \operatorname{Var} \circ a_{2}$, donc $t A_{0}=A_{-1} \circ$ Var dans $D\left(C_{X_{0}}\right)$. Ce qui achève la démonstration.

3.4.12. Proposition (cf. [17], [22]). Si $M$ est holonome, posons $K=D R_{X} M$, on a alors la decomposition de Jordan $T=T_{s} T_{u}$ de la monodromie $T$ de $\psi K$ et de $\phi K$ (voir aussi 3.4.14). Posons

$$
\psi_{\lambda} K=\operatorname{Ker}\left(T_{s}-\lambda: \psi K \rightarrow \psi K\right) \quad\left(\text { de même pour } \phi_{\lambda} K\right) \text {. }
$$

$S i(M, F)$ est quasi-unipotent et régulier le long de $X_{0}$ (et $M$ holonome), alors $A_{\infty}$ et $B_{\beta}$ induisent des isomorphismes

$$
D R_{X_{0}}\left(G r_{a}^{V} M\right) \simeq\left\{\begin{array}{lll}
\psi_{e}(\alpha) K[-1] & \text { si } & -1 \leqslant \alpha<0 \\
\phi_{e(\alpha)} K[-1] & \text { si } & -1<\alpha \leqslant 0
\end{array}\right.
$$

tels que $t \partial_{t}-\alpha, \partial_{t}, t$ sont identifiés avec $N$, can, Var, où $e(\alpha)=\exp 2 \pi i \alpha$.

Preuve. Puisque il existe le polynôme minimal pour l'action de $T$ (au moins localement), on a la décomposition de Jordan, car $\psi K[-1]$ et $\phi K[-1]$ sont des faisceaux pervers. D'après $3.4 .4,3.4 .5$ et $3.4 .8, A_{\alpha}$ et $\oplus B_{\alpha}$ sont des isomorphismes, de plus

$$
\operatorname{Im}\left(B_{\alpha}: V_{0} \tilde{D R_{S}} \psi_{\alpha}^{a l} D R_{X / S} M \rightarrow \widetilde{D R}_{S} \psi D R_{X / S} M \simeq \psi D R_{X} M\right)=\psi_{e(\alpha)} K
$$

(de même pour $\phi)$, car l'action de $t \partial_{t}-\alpha$ sur $\mathscr{H}^{i}\left(\operatorname{Im} B_{\alpha}\right)$ est nilpotente et l'image de $\operatorname{Im} B_{\alpha}$ par la projection $\psi K \rightarrow \psi_{\lambda} K$ est nulle si $\lambda \neq e(\alpha)$ (voir aussi 3.4.14). Puisque 


$$
G r_{a}^{V} D R_{X / s} M \simeq D R_{X_{0}} G r_{a}^{V} M
$$

l'assertion résulte de 3.4.11.

3.4.13. Remarque. Soient $f: X \rightarrow Y$ un morphisme propre, $q: Y \rightarrow S$ un morphisme lisse, tels que $p=q f$ soit lisse. Soit $(M, F)$ un $\mathscr{D}_{X}$-Module holonome filtré, quasi-unipotent et régulier le long de $X_{0}$. Si $f_{*}(M, F, V)$ est strict, et si, de plus, $\mathscr{H}^{i} f_{*}(M, F, V)$ sont quasi-unipotents et réguliers le long de $Y_{0}, 3.4 .12$ (pour $(M, F)$ ) induit un isomorphisme

$$
D R_{Y_{0}}\left(G r_{a}^{V} \mathscr{H}^{i} f_{*} M\right) \simeq \psi_{e(\alpha)}^{p} \mathscr{H}^{i} f_{*} D R_{X} M[-1] \quad(\text { ou } \phi)
$$

(car $D R_{Y_{0}}\left(G r_{a}^{V} f_{*} M\right) \simeq \psi_{e(\alpha)} f_{*} D R_{X} M[-1]$ (ou $\left.\phi\right)$ ) qui coïncide avec l'isomorphisme donné par 3.4.12 appliquée à $\mathscr{H}^{i} f_{*}(M, F)$ (notons que $D R_{Y} \mathscr{H}^{i} f_{*} M=$ $\left.{ }^{p} \mathcal{H}^{i} f_{*} D R_{X} M\right)$, car les morphismes $A_{\alpha}$ et $B_{\beta}$ sont compatibles avec l'image directe par un morphisme propre.

3.4.14. Remarque. Soit $X$ une variété complexe. Soit $D_{c}\left(A_{X}, T\right)$ la catégorie dérivée des complexes des $A_{X}$-Modules munis d'un automorphisme $T$, cohomologiquement constructibles, où $A$ est un corps. Soit $\tilde{A}=A\left[T, T^{-1}\right]$, alors cette catégorie est équivalente à $D_{c}\left(\tilde{A_{X}}\right)$ la catégorie dérivée des complexes des $\tilde{A_{X^{-}}}$Modules, cohomologiquement constructible sur $A$. D'après [33], celle-ci est équivalente à la sous-catégorie pleine $D_{c}\left(\tilde{A_{X}}\right)_{\mathrm{inj}}$ des complexes des $\tilde{A_{X}}$-Modules injectifs. Pour $P$ un polynôme unitaire irréductible sur $A$, tel que $P(0) \neq 0$, on définit le foncteur:

$$
{ }_{P}(\quad): D_{c}\left(\tilde{A_{X}}\right)_{\mathrm{inj}} \rightarrow D\left(\tilde{A_{X}}\right)
$$

par ${ }_{P} K=\bigcup_{i} \operatorname{Ker}\left(P(T)^{i}: K \rightarrow K\right)$ pour $K \in D_{c}\left(\tilde{A_{X}}\right)_{\mathrm{inj}}$. Alors on a ${ }_{P} K \in D_{c}\left(\tilde{A_{X}}\right)$ et le morphisme canonique: $\oplus_{P} K \rightarrow K$ est un isomorphisme dans $D_{c}\left(\tilde{A_{X}}\right)$. (Notons que le foncteur $K \mapsto_{P} K$ est bien défini, si $P: K^{j} \rightarrow K^{j}$ sont surjectifs.)

En effet, soit $W$ la filtration canonique $\tau$ de $K$, alors par la résolution de Cartan-Eilenberg, on a une resolution filtrée: $(K, W) \underset{\rightarrow}{\rightarrow}(L, W)$ telle que $G r_{j}^{W} L$ soient des complexes bornés de $\tilde{A_{X}}$-Modules injectifs; donc on peut supposer que $\mathscr{H}^{j} K=0$ pour $j \neq 0$, et de plus que $\mathscr{H}^{0} K \simeq j_{1} L$ pour $L$ un système local sur $U$ un ouvert de Zariski lisse de $X$, avec $j: U \rightarrow X$ l'inclusion naturelle (par dévissage). Puisque ${ }_{p} K$ est acyclique si $K$ l'est, on peut supposer que $U=X$, et de plus que $X=p t$ avec $L$ un $\tilde{A}$-module irreductible, car l'assertion est locale (et par dévissage); on a donc $L=\tilde{A} / \tilde{A} Q$ pour $Q$ un polynôme unitaire irréductible tel que $Q(0) \neq 0$, alors ${ }_{P} K=0(P \neq Q)$ est clair, donc il suffit de vérifier que ${ }_{Q} K \underset{\rightarrow}{\rightarrow}$. Soient $S=\operatorname{Spec} \tilde{A}, q=(Q) \in S, B=\mathcal{O}_{S, q}$ et $F$ le corps des fractions de 
$B$, alors $B$ est un anneau de valuation discrète muni d'une uniformisante $Q$; on a donc une suite exacte:

$$
0 \rightarrow L \rightarrow F / B \stackrel{Q}{\rightarrow} F / B \rightarrow 0,
$$

d'où l'assertion, $\operatorname{car}_{Q}(F / B)=F / B$.

On applique l'assertion à $\psi K($ ou $\phi K)$ avec $T$ la monodromie. On obtient la décomposition $\psi K=\bigoplus_{P}(\psi K)$ et l'action de $N$ :

$$
N: \psi K \rightarrow \psi K(-1) \quad(\text { de même pour } \phi) \text { 。 }
$$

Notons que l'action de $P(T)$ sur $i^{-1} R j_{*} j^{-1} L$ (cf. 3.4.3) est surjective, si $L$ est un faisceau flasque (et si $P(0) \neq 0$ ).

\subsection{Le cas non-caractéristique}

3.5.1. Soit $f: X \rightarrow Y$ un morphisme de variétés complexes lisses, soit $(M, F)$ un $\mathscr{D}_{Y}$-Module filtré cohérent. On dit que $f$ est non-caractéristique pour $(M, F)$ (ou $(M, F)$ l'est pour $f$ ) si les conditions suivantes sont satisfaites:

$$
\mathscr{H}^{i}\left(f^{-1} G r^{F} M \underset{f^{-1} \mathscr{O}_{Y}}{\operatorname{L}} \mathcal{O}_{X}\right)=0 \text { pour } \quad \mathrm{i} \neq 0,
$$

le morphisme $d f^{*}:\left(p r_{2}\right)^{-1} \operatorname{Car}(M) \rightarrow T^{*} X$ soit fini, où $p r_{2}: X \underset{Y}{X} T^{*} Y \rightarrow T^{*} Y$ est la projection naturelle et $d f^{*}: \underset{Y}{X} T^{*} Y \rightarrow T^{*} Y$ est défini par $d f^{*}(x, \xi)=$ $d f^{* \xi}$ pour $x \in X, \xi \in T_{f(x)}^{*} Y$.

On dit que $f$ est strictement non-caractéristique pour $(M, F)$ si la condition (3.5.1.1) et la condition suivante sont satisfaites:

$$
\begin{aligned}
& \text { il existe une stratification de Whitney }\left\{Y_{\alpha}\right\} \text { be } Y \\
& \text { telle que } \operatorname{Car}(M) \subset \bigcup_{\infty} T_{Y_{\alpha}}^{*} Y \text { et que } \\
& d f^{*}:\left(p r_{2}\right)^{-1}\left(\bigcup_{\infty} T_{Y_{\alpha}}^{*} Y\right) \rightarrow T^{*} X \text { soit fini . }
\end{aligned}
$$

Notons que la condition (3.5.1.3) entraîne que $M$ est holonome.

Lorsque $f$ est une immersion fermée, $X$ est dite (strictement) non-caractéristique pour $(M, F)$ si $f$ l'est.

Si $f$ est non-caractéristique pour $(M, F)$, on définit

$$
\begin{aligned}
& f^{*}(M, F)=(\tilde{M}, F)[-d], \\
& f^{\prime}(M, F)=f^{*}(M, F)(d)[2 d]
\end{aligned}
$$

$\operatorname{par} \tilde{M}=f^{-1} M \bigotimes_{f^{-1} \Theta_{Y}} \omega_{X / Y}, F_{p} \tilde{M}=f^{-1} F_{p+d} M \bigotimes_{f^{-1} \Theta_{Y}} \omega_{X / Y}(p \in \mathbb{Z})$ et $d=\operatorname{dim} X-$ 
$\operatorname{dim} Y$, où $(M, F)(d)=(M(d), F[d])$ et $M(d)=M \otimes Z(d)$.

3.5.2. Lemme. Si $f$ est lisse, $(M, F)$ est non-caractéristique pour $f$.

(C'est clair, car $\mathcal{O}_{X}$ est plat $\operatorname{sur} \mathcal{O}_{Y}$ et $d f^{*}: \underset{Y}{X} T^{*} Y \rightarrow T^{*} Y$ est injectif.)

3.5.3. Lemme. Si $f$ est non-caractéristique pour $(M, F), f^{*}(M, F)[d]$ est un $\mathscr{D}_{X}$-Module filtré cohérent tel que

$$
\operatorname{Car}\left(f^{*} M[d]\right)=d f^{*}\left(p r_{2}^{-1} \operatorname{Car}(M)\right) .
$$

Preuve. On a par définition

$$
\left.p r_{2}^{-1} \operatorname{Car}(M)=\operatorname{supp}\left(f^{-1} G r^{F} M \underset{f^{-1} O_{Y}}{\otimes} \mathcal{O}_{X}\right)^{\sim} \subset X \underset{Y}{X} T^{*} Y \quad \text { (= Specan } G r \mathscr{D}_{X \rightarrow Y}\right)
$$

et

$$
\operatorname{Car}\left(f^{*} M[d]\right)=\operatorname{supp}\left(f^{-1} G r^{F} M \underset{f^{-1} \mathcal{O}_{Y}}{\otimes} \mathcal{O}_{X}\right)^{\sim} \subset T^{*} X \quad\left(=\text { Specan } G r \mathcal{D}_{X}\right)
$$

où $f^{-1} G r^{F} M \underset{f^{-1} \Theta_{X}}{\otimes} \mathcal{O}_{X}$ est muni d'une structure de $G r \mathscr{D}_{X}$-Module par le morphisme d'anneaux $d f_{*}: \operatorname{Gr} \mathscr{D}_{X} \rightarrow G r \mathscr{D}_{X \rightarrow Y}$ et est cohérent sur $\operatorname{Gr} \mathscr{D}_{X}$ par la condition (3.5.1.2); alors l'assertion est claire, car $d f^{*}=\operatorname{Specan}\left(d f_{*}\right)$.

3.5.4. Lemme. Soient $f: X \rightarrow Y$ et $g: Y \rightarrow Z$ des morphismes de variétés complexes lisses, soit $(M, F)$ un $\mathscr{D}_{Z}$-Module filtré cohérent, alors:

(1) $S i(M, F)$ et $g^{*}(M, F)[\operatorname{dim} Y-\operatorname{dim} Z]$ sont non-caractéristique respectivement pour $g$ et $f,(M, F)$ l'est pour $g f$; on a la réciproque (au voisinage de $X)$ si $f$, $g$ sont des immersions fermees.

(2) Supposons que $g$ soit lisse, alors $g f$ est non-caractéristique pour $(M, F)$ sii $f$ l'est pour $g^{*}(M, F)[\operatorname{dim} Y-\operatorname{dim} Z]$.

Preuve. La première assertion est claire; la réciproque résulte de la théorie de suites régulières car $N \bigotimes_{O_{Z}}^{\boldsymbol{L}} \mathcal{O}_{X} \simeq K\left(N ; z_{1}, \cdots, z_{n}\right)[n]$. Si $g$ est lisse, id $\times d g^{*}$ : $\underset{z}{X} T^{*} Z \rightarrow \underset{Y}{X} T^{*} Y$ est une immersion fermée et $\mathcal{O}_{Y}$ est plat sur $\mathcal{O}_{z}$; alors l'assertion résulte des diagrammes suivants:

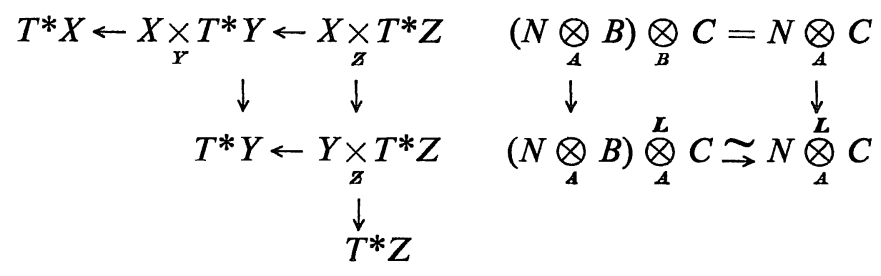

où $A=\mathcal{O}_{Z}, B=\mathcal{O}_{Y}$ et $C=\mathcal{O}_{X}$. 
3.5.5. Lemme. Soit $(M, F) \in M F_{h}\left(\mathscr{D}_{Y}\right)$, i.e. $M$ est holonome. Si $f$ est noncaractéristique pour $(M, F)$, on a $f^{*}(M, F)[d] \in M F_{h}\left(\mathscr{D}_{X}\right)$.

Preuve. Si $f$ est lisse, on a $G r f^{*}(M, F)[d] \simeq f^{-1} G r M \underset{f^{-1} \Theta_{Y}}{\otimes} \omega_{X / Y}$; donc $\operatorname{dim} \operatorname{Car}\left(f^{*}(M, F)[d]^{\sim}=\operatorname{dim} X\right.$. Si $f$ est une immersion fermée telle que $\operatorname{dim} X=\operatorname{dim} Y-1$, on a (localement)

$$
\operatorname{dim} \operatorname{Car} f^{*}(M, F)[d]<\operatorname{dim} X \quad \text { si } \quad \operatorname{Car}(M) \mp X \underset{Y}{X} T^{*} Y
$$

et

$$
\operatorname{Car}(M)=\left(d f^{*}\right)^{-1} d f^{*} \operatorname{Car}(M) \text { sinon, }
$$

car $\operatorname{Car}(M)$ est Lagrangienne; d'où l'assertion. Le cas général résulte de 3.5.4.

3.5.6. Lemme. Soit $i: X \hookrightarrow Y$ une immersion fermée de variétés complexes lisses telles que $\operatorname{dim} X=\operatorname{dim} Y-1$. Soit $(M, F)$ un $\mathscr{D}_{Y}$-Module filtré cohérent. Si $X$ est non-caractéristique pour $(M, F)$, alors $(M, F)$ est quasi-unipotent et régulier le long de $X$, de plus la filtration $V$ est définie par

$$
V_{\alpha} M=\left\{\begin{array}{lll}
M \mathcal{G}_{X}^{i} & \text { si } & -i-1 \leqslant \alpha<-i, i \geqslant 0, \\
M & \text { si } \alpha \geqslant 0
\end{array}\right.
$$

où $\mathcal{G}_{X}$ est l'Idéal de $X$; en particulier on a un isomorphisme canonique

$$
G r_{-1}^{V}(M, F) \otimes \omega_{X / Y} \simeq i^{!}(M, F)[1]
$$

et $\operatorname{Gr}_{\alpha}^{V}(M, F)=0$ pour $\alpha \neq-1,-2, \cdots$.

Preuve. On définit la filtration $V$ par (3.5.6.1). Puisque $t: G r^{F} M \rightarrow G r^{F} M$ est injectif par la condition (3.5.1.1), on a l'injectivité de $t^{i}: M / F_{p} M \rightarrow M / F_{p} M$ et de $t^{i}: M / F_{p} M \rightarrow V_{-i-1} M / F_{p} V_{-i-1} M$, alors on vérifie que $t^{i}: F_{p} M \rightarrow F_{p} V_{-i-1} M$ est bijectif par le diagramme:

$$
\begin{aligned}
& 0 \rightarrow F_{p} M \longrightarrow M \longrightarrow M / F_{p} M \longrightarrow 0 \\
& t^{i} \downarrow \quad t^{i} \downarrow \quad t^{i} \downarrow \\
& 0 \rightarrow F_{p} V_{-i-1} M \rightarrow V_{-i-1} M \rightarrow V_{-i-1} M / F_{p} V_{-i-1} M \rightarrow 0 \text {. }
\end{aligned}
$$

Il reste à démontrer que $G r^{F} M$ sont cohérents sur $G r^{F} V_{0} \mathscr{D}_{Y}$. Posons

$$
\begin{aligned}
& T^{*}(Y / \boldsymbol{C})=\operatorname{Coker}\left(d t^{*}: \underset{\boldsymbol{C}}{Y \times} T^{*} \boldsymbol{C} \hookrightarrow T^{*} Y\right) \\
& \phi: T^{*} Y \rightarrow T^{*}(Y / \mathbb{C}) \quad \text { la projection naturelle, }
\end{aligned}
$$

alors $\operatorname{Car}(M)$ est finie sur $T^{*}(Y / C)$ au voisinage de $X \underset{Y}{\times} T^{*}(Y / C)=T^{*} X$ par la condition (3.5.1.2), donc $G r^{F} M$ est cohérent sur $G r^{F} \mathscr{D}_{Y / C}$ et sur $G r^{F} V_{0} \mathscr{D}_{Y}$. 
3.5.7. Lemme. Soient $X, Y$ et $(M, F)$ comme dans 3.5 .6 (en particulier $X$ est non-caractéristique pour $(M, F))$, soit $M(* X)$ la localisation de $M$ par $t$, on a alors une filtration $F$ sur $M(* X)$ telle que $(M(* X), F)$ soit un $\mathscr{D}_{Y}$-Module filtré cohérent et qu'on ait une suite exacte filtrée

$$
0 \rightarrow(M, F) \rightarrow(M(* X), F) \rightarrow i_{*} i^{!}(M, F)[1] \rightarrow 0 ;
$$

de plus $(M(* X), F)$ est quasi-unipotent et régulier le long de $X$ et la filtration $V$ est définie par

$$
V_{\alpha} M(* X)=M t^{i} \text { pour } \quad-i-1 \leqslant \alpha<-i .
$$

Preuve. Soit $\tilde{M}=M(* X)$. On définit la filtration $F$ par

$$
\begin{aligned}
& F_{p}\left(M t^{-1}\right)=\left(F_{p} M\right) t^{-1}, \\
& F_{p}(\tilde{M})=\sum_{i \geqslant 0}\left(F_{p-i}\left(M t^{-1}\right)\right) \partial_{t}^{i}
\end{aligned}
$$

et $V$ par (3.5.7.2). D'abord on vérifie que $t: G r_{i}^{V} \tilde{M} \rightarrow G r_{i-1}^{V} \tilde{M}$ sont bijectifs et que l'action de $t \partial_{t}-i$ sur $G r_{i}^{V} \tilde{M}$ est nulle. Par le même argument que dans la epreuv de 3.2 .2 , on vérifie que $\partial_{t}: G r_{\alpha}^{V} F_{p} \tilde{M} \rightarrow G r_{\alpha+1}^{V} F_{p+1} \tilde{M}$ sont bijectifs pour $\alpha \geqslant 0$; en particulier on a un isomorphisme canonique

$$
(M(* X) / M, F) \simeq i_{* i} i^{!}(M, F)[1]
$$

car $\left(G r_{0}^{V} M, F\right) \simeq i^{!}(M, F)[1]$ par définition; donc on a (3.5.7.1). Les autres assertions sont claires.

3.5.8. Lemme. Soient $X, Y$ et $(M, F)$ comme dans 3.5.7. On définit un $\mathscr{D}_{Y}$-Module par $M(! X):=M \oplus i_{*} i * M[-1]$ avec

$$
(m, n) P=(m P, n P) \quad \text { pour } \quad P \in V_{0} \mathscr{D}_{Y}
$$

et

$$
(m, n) \partial_{t}=\left(m \partial_{t},[m] \otimes\left[\partial_{t}\right]+n \partial_{t}\right)
$$

ou $[m] \otimes\left[\partial_{t}\right] \in M / M t \otimes \omega_{X / Y} \subset i_{*} i^{*} M[-1]$, et la filtration $F$ par

$$
F_{p}(M(! X))=F_{p} M \oplus F_{p}\left(i_{*} i^{*} M[-1]\right),
$$

de sorte qu'on ait une suite exacte filtrée:

$$
0 \rightarrow i_{*} i^{*}(M, F)[-1] \rightarrow(M(! X), F) \rightarrow(M, F) \rightarrow 0 ;
$$

alors $(M(! X), F)$ est quasi-unipotent et régulier le long de $X$ et la filtration $V$ est définie par

$$
V_{\alpha}(M(! X))=V_{\alpha} M \oplus V_{\alpha}\left(i_{*} i^{*} M[-1]\right)
$$


Preuve. Puisque $\mathscr{D}_{Y}=\mathscr{D}_{Y / C} \otimes C\left[\partial_{t}\right]$ (localement) et $(m t, n t) \partial_{t}=\left(m t \partial_{t}, n t \partial_{t}\right)$, on vérifie que la structure de $\mathscr{D}_{Y}$-Module est bien définie et indépendente du choix de $\partial_{t}$, alors les autres assertions sont claires.

3.5.9. Lemme. Soient $X, Y$ et $(M, F)$ comme dans 3.5.7, soit $j: Y \backslash X \hookrightarrow Y$ l'inclusion naturelle. Supposons que $(M, F)$ soit holonome, on a alors les isomorphismes

$$
\begin{aligned}
& D R(M(* X)) \simeq R j_{*} j^{*} D R M, \quad D R i^{!} M \simeq i^{!} D R M, \\
& D R(M(! X)) \simeq j_{!} j^{!} D R M, \quad D R i^{*} M \simeq i^{*} D R M,
\end{aligned}
$$

de sorte que les triangles

$$
\begin{aligned}
& \rightarrow D R M \rightarrow D R M(* X) \rightarrow D R i_{*} i^{!} M[1] \stackrel{+1}{\longrightarrow} \\
& \rightarrow D R i_{*} i^{*} M[-1] \rightarrow D R M(! X) \rightarrow D R M \stackrel{+1}{\longrightarrow}
\end{aligned}
$$

induits par (3.5.7.1) et (3.5.8.1) sont identifiés avec les triangles

$$
\begin{aligned}
& \rightarrow i_{1} i^{!} D R M \rightarrow D R M \rightarrow R j_{*} j^{*} D R M \stackrel{+1}{\longrightarrow} \\
& \rightarrow j_{!} j^{!} D R M \rightarrow D R M \rightarrow i_{*} i^{*} D R M \stackrel{+1}{\longrightarrow}
\end{aligned}
$$

au décalage près.

Preuve. Soit $M \rightarrow I^{\bullet}$ une resolution injective, posons

$$
\boldsymbol{R} j_{*} j^{*} D R M^{\bullet}=j_{*} j^{*} D R I^{\bullet}
$$

où $D R$ signifie le foncteur $\otimes_{\mathcal{O}_{Y}} \wedge^{-\cdot \Theta_{Y}}$; on a alors le morphisme canonique

$$
D R M(* X) \rightarrow \boldsymbol{R} j_{*} j^{*} D R M
$$

défini par

$$
D R M(* X) \rightarrow D R I^{*}(* X) \rightarrow D R j_{*} j^{*} I^{\bullet}=j_{*} j^{*} D R I^{\bullet},
$$

de plus le diagramme

$$
\stackrel{D}{D R M(* X)} \stackrel{\searrow}{\longrightarrow} \mathrm{R}_{* j} j^{*} D R M
$$

commute dans la catégorie des $\boldsymbol{C}$-complexes; donc il suffit de vérifier que (3.5.9.1) est un quasi-isomorphismes. Puisque l'assertion est locale, on peut supposer que $Y=X \times S$ et que $t$ est une coordonnée de $S \subset C$, on a alors

$$
D R_{Y} M(* X)=D R_{S}\left(D R_{Y / S} M(* X)\right) \simeq D R_{S}\left(D R_{Y / S} I^{*}(* X)\right)
$$


Posons $L^{*}=D R_{Y / S} I^{*}$, alors il suffit de vérifier que

$$
D R_{S}(L(* X)) \rightarrow D R_{S}\left(\boldsymbol{R} j_{*} j^{*} L^{*}\right)
$$

est un quasi-isomorphisme. Par le même argument que la preuve de 3.4.5 (i.e. en utilisant la fibration de Milnor), on peut suppose que $Y=S$ et que $\mathscr{H}^{i} L^{\bullet}$ sont des $\mathscr{D}_{S}$-Modules holonomes réguliers, car $(M, F)$ est holonome et quasiunipotent et régulier le long de $X$, cf. la preuve de 3.4.9. En utilisant la filtration canonique, on peut supposer que $L$ est un Module, alors l'assertion est bien connue.

Maintenant démontrons les assertion pour $D R M(! X)$. Puisque $j^{!}$est un foncteur adjoint à droite de $j_{!}$, on a le morphisme canonique

$$
j_{!} j^{!} D R M \rightarrow D R M(! X)
$$

tel que le diagramme

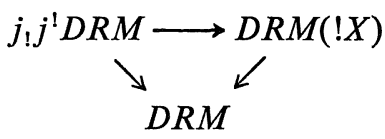

commute dans la catégorie des $\boldsymbol{C}$-complexes, donc il suffit de vérifier que (3.5.9.2) est un quasi-isomorphisme, i.e. $i^{*} D R M(! X)=0$. Posons $L=$ $D R_{Y / S} M(! X)$ comme ci-dessus. Puisque $M(! X)$ est holonome et quasi-unipotent et régulier le long de $X, H^{i} L_{x}$ sont des $\mathscr{D}_{S, 0}$-modules holonomes réguliers pour $i \in \boldsymbol{Z}, x \in X$. Puisque

$$
\partial_{t}: G r_{-1}^{V} M(! X) \rightarrow G r_{0}^{V} M(! X)
$$

est bijectif, on obtient que

$$
\partial_{t}: G r_{-1}^{V} H^{i} L_{x} \rightarrow G r_{0}^{V} H^{i} L_{x}
$$

sont bijectifs, donc $\operatorname{DRM}(! X)_{x}=D R_{S} L_{x}^{*}$ est acyclique pour $x \in X$. Ce qui achève la démonstration de 3.5.9.

3.5.10. Définition. Soient $X, Y, i$ et $(M, F)$ comme dans 3.5.7, alors le morphisme de Gysin (resp. de restriction)

$$
i_{\sharp}: i_{*} i^{!}(M, F) \rightarrow(M, F) \quad\left(\text { resp. } i^{\sharp}:(M, F) \rightarrow i_{*} i^{*}(M, F)\right)
$$

est un morphisme dans $D F\left(\mathscr{D}_{Y}\right)$ défini par le triangle (3.5.7.1) (resp. (3.5.8.1)).

3.5.11. Soit $Y=Y_{1} \cup Y_{2}$ un diviseur à croisements normaux sur $X$ tel que $Y_{1}$ et $Y_{2}$ soient lisses et que $Y_{1}, Y_{2}$ et $Z=Y_{1} \cap Y_{2}$ soient non-caractéristiques pour un $\mathscr{D}_{X}$-Module filtré cohérent $(M, F)$. Soit $f=x y$ où $x$ (resp. $y$ ) est un généra- 
teur de l'Idéal de $Y_{1}\left(\operatorname{resp} . Y_{2}\right)$. Posons $(\tilde{M}, F)=i_{f^{*}}(M, F)$, on a par définition:

(3.5.11.1) $\tilde{M}=M \otimes C\left[\partial_{t}\right], \quad F_{p} \tilde{M}=\oplus F_{p-j} M \otimes \partial_{t}^{j}$

$$
\begin{array}{ll}
\left(u \otimes \partial_{t}^{j}\right) \xi=u \xi \otimes \partial_{t}^{j}-u(\xi f) \otimes \partial_{t}^{j+1}, & \left(u \otimes \partial_{t}^{j}\right) a=u a \otimes \partial_{t}^{j}, \\
\left(u \otimes \partial_{t}^{j}\right) t=u f \otimes \partial_{t}^{j}+j u \otimes \partial_{t}^{j-1}, & \left(u \otimes \partial_{t}^{j}\right) \partial_{t}=u \otimes \partial_{t}^{j+1}, \\
\text { pour } u \in M, \xi \in \Theta_{X}, a \in \mathcal{O}_{X} . &
\end{array}
$$

Soit $s=t \partial_{t}$, on définit les filtrations $V, \mathbb{V}$ et $G$ par

$$
V_{\alpha} \tilde{M}=\left\{\begin{array}{lc}
\left(M f^{i} \otimes 1\right) \mathscr{D}_{X}, & -i-1 \leqslant \alpha<-i, i \geqslant 0 \\
\sum_{0 \leqslant l \leqslant \alpha+1}\left(V_{-1} \tilde{M}\right) \partial_{l}, & \alpha \geqslant 0 .
\end{array}\right.
$$

(3.5.11.3) $\quad \mathbb{V}_{-i-1, k} \tilde{M}= \begin{cases}V_{-i-2} \tilde{M}, & i \geqslant 0, k \leqslant-2 \\ V_{-i-1} \tilde{M}(s+i+1)+V_{-i-2} \tilde{M}, & i \geqslant 0, k=-1 \\ \left(M f^{i}(x, y) \otimes 1\right) \mathscr{D}_{X}, & i \geqslant 0, k=0 \\ V_{-i-1} \tilde{M}, & i \geqslant 0, k \geqslant 1\end{cases}$

$$
G_{q} \tilde{M}=\sum_{j \leqslant q} \otimes \partial_{t}^{j}
$$

Soient $\partial_{x}, \partial_{y}$ des champs de vécteur (loc. définis) sur $X$ tel que

$$
\left[\partial_{x}, x\right]=\left[\partial_{y}, y\right]=1,\left[\partial_{x}, y\right]=\left[\partial_{y}, x\right]=0 .
$$

Puisque $(u \otimes 1) x \partial_{x}=u x \partial_{x} \otimes 1-(u \otimes 1) s$, on vérifie que $V_{\alpha} \tilde{M}$ et $\mathbb{V}_{-i-1, k} \tilde{M}$ sont des $V_{0} \mathscr{D}_{X \times C}$-sous-Modules de $\tilde{M}$; de plus ce sont des $V_{0} \mathscr{D}_{X \times C}$-Modules cohérents, car $M$ est de type fini sur $\mathscr{D}_{X / C^{2}}$ (resp. $\mathscr{D}_{X / C}$ ) au voisinage de $Z$ (resp. $Y \backslash Z$ ) par la condition non-caractéristique. On a $\mathbb{V}_{i, k} \subset \mathbb{V}_{i, k+1}$ car

$$
\begin{aligned}
& \left(u f^{i} \otimes 1\right)(s+i+1)=\left(u \partial_{y} f^{i} y \otimes 1\right)-\left(u f^{i} y \otimes 1\right) \partial_{y} \\
& \text { (de même pour } \left.\partial_{x}\right) .
\end{aligned}
$$

Soient $i: Z \hookrightarrow X, i_{p}: Y_{p} \hookrightarrow X(p=1,2)$ les inclusions naturelles, on définit des morphismes $\mathscr{D}_{X}$-linéaires filtrés

$$
\begin{aligned}
& \alpha_{i,-1}: i_{*} i^{!}(M, F[1])[2] \rightarrow\left(G r_{i,-1}^{V} \tilde{M}, F\right) \\
& \alpha_{i, 0}: \bigoplus_{p} i_{p^{*}} i_{p}^{1}(M, F)[1] \rightarrow\left(G r_{i, 0}^{V} \tilde{M}, F\right) \\
& \alpha_{i, 1}: i_{*} i^{1}(M, F)[2] \rightarrow\left(G r_{i, 1}^{V} \tilde{M}, F\right)
\end{aligned}
$$

pour $i<0$ par

$$
\begin{aligned}
& \alpha_{i,-1}(u)=\left(u f^{-i} \otimes 1\right)(s-i) \\
& \alpha_{i, 0}\left(u_{1}, u_{2}\right)=\left(u_{1} f^{-i}+u_{2} f^{-i}\right) \otimes 1, \\
& \alpha_{i, 1}(u)=u f^{-i} \otimes 1,
\end{aligned}
$$


où $u \in i^{!} M[2]=M f^{-1} / M x^{-1}+M y^{-1}, u_{1} \in i_{1}^{!} M[1]=M x^{-1} / M$ et $u_{2} \in i_{2}^{!} M[1]=$ $M y^{-1} / M$. Notons que $\alpha_{i, k}$ sont uniquement déterminés par leur restriction sur $i^{!}(M, F[1])[2]$ etc. On vérifie que $\alpha_{i, k}$ sont bien-définis (e.g. $\alpha_{i,-1}\left(M x^{-1}\right) \subset$ $V_{i,-2} \tilde{M}$ et $\alpha_{i, 0}$ est $\mathscr{D}_{X}$-linéaire) par la formule (3.5.11.5).

Sur $X \backslash Z$ il existe (localement) un champ de vecteur $\partial_{f}$ tel que $\left[\partial_{f}, f\right]=1$ au voisinage de $Y$. Puisque

on vérifie que

$$
(u \otimes 1) \partial_{f}=u \partial_{f} \otimes 1-u \otimes \partial_{t}
$$

$$
\begin{aligned}
& V_{\alpha} \tilde{M}= \begin{cases}\tilde{M} t^{i} & -i-1 \leqslant \alpha<-i, \quad i \geqslant 0 \\
\tilde{M} & \alpha \geqslant 0\end{cases} \\
& V_{i, k} \tilde{M}= \begin{cases}V_{i-1} \tilde{M} & k<0 \\
V_{i} \tilde{M} & k \geqslant 0,\end{cases}
\end{aligned}
$$

donc on va considérer seulement au voisinage de $Z$ où $\partial_{x}, \partial_{y}$ existent (localement), et tel que $G r^{F} M$ soit plat sur $C_{x, y}^{2}$ et de type fini sur $G r^{F} \mathscr{D}_{X / C^{2}}$.

3.5.12. Lemme.

$$
G_{q} \boldsymbol{V}_{-i-1, k} \tilde{M}= \begin{cases}\left(M f^{i+1} \otimes 1\right) F_{q} \mathscr{D}_{X}, & k \leqslant-2 \\ \left(M f^{i} \otimes 1\right)(s+i+1) F_{q-1} \mathscr{D}_{X}+M f^{i+1} \otimes 1, & k=-1 \\ \left(M f^{i}(x, y) \otimes 1\right) F_{q} \mathscr{D}_{X}, & k=0 \\ \left(M f^{i} \otimes 1\right) F_{q} \mathscr{D}_{X}, & k \geqslant 1 .\end{cases}
$$

Preuve. Puisque $\{x, y\}$ est une suite régulière de $G r^{F} M$ et de $M$, on a:

(3.5.12.1) Soit $P(S, T)=\sum u_{i} S^{i} T^{q-i} \in M[S, T]$ tel que $P(x, y)=0$, alors il existe $Q(S, T)=\sum u_{i}^{\prime} S^{i} T^{q-1-i}$ tel que $P(S, T)=Q(S, T)(y S-x T)$, i.e. $u_{i}=u_{i-1}^{\prime} y-u_{i}^{\prime} x$.

D'abord on va démontrer

$$
G_{q-1} \tilde{M} \cap\left(M f^{i} \otimes 1\right) F_{q} \mathscr{D}_{X}=\left(M f^{i} \otimes 1\right) F_{q-1} \mathscr{D}_{X} \quad(i \geqslant 0),
$$

ce qui entraine l'assertion pour $k \leqslant-2$ et $k \geqslant 1$. Puisque

$$
\left(M f^{i} \otimes 1\right) F_{q} \mathscr{D}_{X}=\sum_{i+j \leqslant q}\left(M f^{i} \otimes 1\right) \partial_{x}^{i} \partial_{y}^{j}
$$

il suffit de vérifier:

(3.5.12.2) Si $u=\sum\left(u_{j} \otimes 1\right) \partial_{x}^{j} \partial_{y}^{q-j} \in G_{q-1} M$ avec $u_{j} \in M f^{i}$, on a $u=\left(v_{j} \otimes 1\right) \partial_{x}^{j} \partial_{y}^{q-1-j}$ avec $v_{j} \in M f^{i}$.

D'après (3.5.11.1), on a $\sum u_{j} y^{j} x^{q-j}=0$, donc (3.5.12.1) entraîne que $u_{j}=$ 
$u_{j-1}^{\prime} x-u_{j}^{\prime} y$ avec $u_{j}^{\prime} \in M f^{i}$, on a alors

$$
\begin{aligned}
u & =\sum\left(\left(u_{j-1}^{\prime} x-u_{j}^{\prime} y\right) \otimes 1\right) \partial_{x}^{j} \partial_{y}^{q-j} \\
& =\sum\left(\left(u_{j}^{\prime} x \partial_{x}-u_{j}^{\prime} y \partial_{y}\right) \otimes 1\right) \partial_{x}^{j} \partial_{y}^{q-1-j}
\end{aligned}
$$

d'où l'assertion (3.5.12.2).

D'après (3.5.11.5) on vérifie que $M f^{i+1} \otimes 1+V_{-i-1} \tilde{M}(s+i+1)$ est un $\mathscr{D}_{X^{-}}$ Module, donc

$$
V_{-i-1,-1} \tilde{M}=M f^{i+1} \otimes 1+V_{-i-1} \tilde{M}(s+i+1) .
$$

Par la même démonstration, on a l'assertion pour $k=-1$, car

$$
s+i+1: G r_{q-1}^{G} \tilde{M} \rightarrow G r_{q}^{G} \tilde{M}
$$

est injectif. Démontrons le cas $k=0$. Supposons que

$$
u=\sum\left(\left(u_{j}^{\prime} x+u_{j}^{\prime \prime} y\right) \otimes 1\right) \partial_{x}^{j} \partial_{y}^{q-j} \in G_{q-1} \tilde{M} \quad \text { avec } \quad u_{j}^{\prime}, u_{j}^{\prime \prime} \in M f^{i},
$$

i.e. $\sum\left(u_{j}^{\prime} y^{j} x^{q-j+1}+u_{j}^{\prime \prime} y^{j+1} x^{q-j}\right)=0$. D'après (3.5.12.1), on a

$$
u_{j}^{\prime}+u_{j-1}^{\prime \prime}=v_{j-1} x-v_{j} y \quad \text { avec } \quad v_{j} \in M f^{i}
$$

donc

$$
\begin{aligned}
u & =\sum\left(\left(-u_{j-1}^{\prime \prime} x+v_{j-1} x^{2}-v_{j} x y+u_{j}^{\prime \prime} y\right) \otimes 1\right) \partial_{x}^{j} \partial_{y}^{q-j} \\
& \left.=\sum\left(\left(-u_{j}^{\prime \prime} x \partial_{x}+u_{j}^{\prime \prime} y \partial_{y}\right)+\left(v_{j} x\right) x \partial_{x}-\left(v_{j} x\right) y \partial_{y}\right) \otimes 1\right) \partial_{x}^{j} \partial_{y}^{q-1-j}
\end{aligned}
$$

d'où l'assertion, car $v f^{i} x \partial_{x}-v f^{i} y \partial_{y}=\left(v \partial_{x}\right) f^{i} x-\left(v \partial_{y}\right) f^{i} y$ pour $v \in M$.

3.5.13. Corollaire. $G r_{q}^{G} \mathbb{V}_{-i-1, k} \tilde{M}=M \underset{A}{\otimes a} a(-i-1, k, q)$, ò̀ $A=C[x, y]$ et

$$
\boldsymbol{a}(-i-1, k, q)= \begin{cases}A f^{i+1}(x, y)^{q} & k \leqslant-2 \\ A f^{i+1}(x, y)^{q-1} & k=-1 \\ A f^{i}(x, y)^{q+1} & k=0 \\ A f^{i}(x, y)^{q} & k \geqslant 1\end{cases}
$$

(C'est clair car $M$ est plat sur $A$.)

3.5.14. Lemme. Les morphismes canoniques

$$
\left(F_{p-q} M\right) a(-i-1, k, q) \otimes \partial_{t}^{q} \hookrightarrow G r_{q}^{G} F_{p} \boldsymbol{V}_{-i-1, k} \tilde{M} \hookrightarrow F_{p} \boldsymbol{V}_{-i-1, k} G r_{q}^{G} \tilde{M}
$$

sont des isomorphismes $(i \geqslant 0)$; en particulier $(F, G, V)$ sont compatibles, $c f$. 1.2.14, et $\left(F_{p} \mathbb{V}_{-i-1, k} \tilde{M}\right) t=F_{p} V_{-i-2, k} \tilde{M}$.

Preuve. Puisque $\left(F_{p-q} M f^{i} \otimes 1\right) F_{q} \mathscr{D}_{X} \subset G_{q} F_{p} V_{-i-1} \tilde{M}$, etc., on vérifie que $\left(F_{p-q} M\right) \boldsymbol{a}(-\mathrm{i}-1, k, q) \otimes \partial_{t}^{q}$ est un sous-Module de $G r_{q}^{G} F_{p} \mathbb{V}_{-i-1, k} \tilde{M}$, donc il 
suffit de démontrer que

$$
\left(F_{p-q} M\right) \boldsymbol{a}(-i-1, k, q) \otimes \partial_{t}^{q}=F_{p} \boldsymbol{V}_{-i-1, k} G r_{q}^{G} \tilde{M} ;
$$

or on a le diagramme commutatif:

$$
\begin{gathered}
0 \rightarrow F_{p-q} M \underset{A}{\otimes a} \rightarrow M \underset{-1}{\otimes} \boldsymbol{a} \rightarrow\left(M / F_{p-q} M\right) \underset{-1}{\otimes} \boldsymbol{a} \rightarrow 0 \\
0 \longrightarrow F_{p-q} M \longrightarrow M \longrightarrow M / F_{p-q} M \rightarrow 0
\end{gathered}
$$

d'où l'assertion.

3.5.15. Corollaire. $\alpha_{-i-1, k}$ sont des isomorphismes filtrés.

Preuve. On définit la filtration $G$ par

$$
\begin{aligned}
& G_{q}\left(i_{*} i^{!} M[2]\right)=\bigoplus_{i+j \leqslant q}\left(M f^{-1} / M x^{-1}+M y^{-1}\right) \partial_{x}^{i} \partial_{y}^{j} \\
& \left.G_{q}\left(i_{1 *} i_{1}^{1} M[1]\right)=\bigoplus_{j \leqslant q}\left(M x^{-1} / M\right) \partial_{x}^{j} \quad \text { (de même pour } i_{2 *} i_{2}^{\prime} M[1]\right),
\end{aligned}
$$

alors les morphismes $\alpha_{-i-1, k}$ préservent les filtrations $G$ (au décalage près si $k=$ -1), donc il suffit de démontrer que $G r_{q}^{G} F_{p} \alpha_{-i-1, k}$ sont des isomorphismes. D'après 3.5.14 on peut supposer que $i=0$, et on a

$$
F_{p} G r_{q}^{G} G r_{-1, k}^{\boldsymbol{V}} \tilde{M}=F_{p-q} M \otimes_{\boldsymbol{A}}(\boldsymbol{a}(-1, k, q) / \boldsymbol{a}(-i, k-1, q)),
$$

avec

$$
\boldsymbol{a}(-1, k, q) / \boldsymbol{a}(-1, k-1, q)= \begin{cases}\bigoplus_{j+k=q-1} \boldsymbol{C} x^{j+1} y^{k+1} & k=-1 \\ A y^{q+1} / A x y^{q+1} \oplus A x^{q+1} / A y x^{q+1} & k=0 \\ \bigoplus_{j+k=q} \boldsymbol{C} x^{j} y^{k} & k=1 .\end{cases}
$$

D'autre part on a

$$
\begin{aligned}
& F_{p} G r_{q}^{G}\left(i_{*} i^{1} M[2]\right)=\bigoplus_{j+k=q}\left(F_{p-q} M f^{-1} / F_{p-q} M x^{-1}+F_{p-q} M y^{-1}\right) \partial_{x}^{j} \partial_{y}^{k} \\
& F_{p} G r_{q}^{G}\left(i_{1 *} i_{1}^{1} M[1]\right)=\left(F_{p-q} M x^{-1} / F_{p-q} M\right) \partial_{x}^{q}
\end{aligned}
$$

et $G r_{q}^{G} \alpha_{-1, k}$ est défini par la substitution $\partial_{x} \rightarrow y, \partial_{y} \rightarrow x$ et par la multiplication de $f$ (ou $f^{2}$ si $k=-1$ ); d'où l'assertion.

3.5.16. Lemme. Soit $i \geqslant 0$, on a alors

$$
V_{i-1} \tilde{M}=\bigoplus_{0 \leqslant j<i} M \otimes \partial_{t}^{j} \oplus V_{-1} \tilde{M} \partial_{t}^{i}, \quad G r_{q}^{G} V_{i-1} \tilde{M}=M(x, y)^{q-i} \otimes \partial_{t}^{q},
$$

et

$$
F_{p-q} M(x, y)^{q-i} \otimes \partial_{t}^{q}=F_{p} V_{i-1} G r_{q}^{G} \tilde{M}=G r_{q}^{G} F_{p} V_{i-1} \tilde{M}
$$


en particulier $(F, V, G)$ sont compatibles.

Preuve. La première assertion résulte de

$$
(M \otimes 1) \partial_{x} \subset(M \otimes 1) \oplus\left(M \otimes \partial_{t}\right), \quad \text { etc. }
$$

On a la deuxième assertion d'après 3.5.12, et les autres assertions par le même argument que la preuve de 3.5.14.

3.5.17. Corollaire. $\partial_{t}^{i}:\left(G r_{-1,1}^{V} \tilde{M}, F\right) \underset{\rightarrow}{\rightarrow}\left(G r_{i-1}^{V} \tilde{M}, F[-i]\right) \quad(i>0)$.

Preuve. On a d'abord $\left(\mathbb{V}_{-i, 0} \tilde{M}\right) \partial_{t} \subset V_{-1} \tilde{M}$, i.e. $(M x \otimes 1) \partial_{t} \subset V_{-1} \tilde{M}$, car $u x \otimes \partial_{t}$ $=-(u \otimes 1) \partial_{y}+\left(u \partial_{y} \otimes 1\right)$; alors l'assertion résulte de

$$
\begin{aligned}
& G r_{q}^{G} F_{p} G r_{-1,1}^{V} \tilde{M}=\left(F_{p-q} M \otimes(x, y)^{q} /(x, y)^{q+1}\right) \otimes \partial_{t}^{q} \\
& G r_{q+i}^{G} F_{k+i} G r_{i-1}^{V} \tilde{M}=\left(F_{p-q} M \otimes(x, y)^{q} /(x, y)^{q+1}\right) \otimes \partial_{t}^{q+i} .
\end{aligned}
$$

3.5.18. Proposition. Soient $(M, F)$ et $f$ comme dans 3.5.11, alors $(M, F)$ est quasi-unipotent et régulier le long de $f$, la filtration $V$ est définie par (3.5.11.2), la filtration $\boldsymbol{V}$ induit la filtration de monodromie $W$ sur $\operatorname{Gr}_{-i-1}^{V} \tilde{M}$ pour $i \geqslant 0$, l'action de $s-\alpha$ est nulle sur $G r_{\alpha}^{V} \tilde{M}$ si $\alpha \geqslant 0$, et (3.2.1.3) est valable pour $\alpha=-1$.

Preuve. D'abord on vérifie que $\left(\boldsymbol{V}_{-i-1, k} \tilde{M}\right)(s+i+1) \subset \boldsymbol{V}_{-i-1, k-2} \tilde{M}$; par exemple, le cas $k=0$ résulte de (3.5.11.5). On a alors

$$
V_{\alpha} \tilde{M}(s-\alpha) \subset V_{\alpha} \tilde{M}, \text { donc } \quad V_{\alpha} \tilde{M} t \subset V_{\alpha-1} \tilde{M} \text { et } \quad V_{\alpha} \tilde{M} \partial_{t} \subset V_{\alpha+1} \tilde{M}
$$

pour tout $\alpha$. D'après 3.5 .15 on a

$$
s+i+1:\left(G r \underline{-i-1,1}_{i} \tilde{M}, F\right) \stackrel{\sim}{\rightarrow}\left(G r r_{i-1,-1} \tilde{M}, F[-1]\right)
$$

et l'action de $s-i+1$ est nulle sur $G r_{i-1}^{V} \tilde{M}$ pour $i>0$ d'après 3.5.17, alors $(M, F)$ est quasi-unipotent et régulier le long de $f$ d'après 3.5 .14 , 3.5.15 et 3.5.17.

\subsection{Le cas croisements normaux}

3.6.1. Soient $X$ une variété complexe lisse, $D$ un diviseur à croisements normaux sur $X$ avec $g$ un générateur global de l'Idéal de $D$. Soient $D^{(i)}=\{x \in X$ : mult $\left._{x} D \geqslant i\right\}, \widetilde{D}^{(i)}$ la normalisation de $D^{(i)}, a_{i}: \widetilde{D}^{(i)} \rightarrow X$ le morphisme naturel. Posons $f=g^{m}$ pour $m>0$. Soit $(M, F)$ un $\mathscr{D}_{X}$-Module holonome filtré tel que chaque $G r_{p}^{F} M$ soit localement libre de type fini sur $\mathcal{O}_{X}$. On définit une autre filtration $G$ par $G_{0} M=M, G_{-1} M=0$. Posons

$$
(\tilde{M} ; F, G)=i_{f *}(M ; F, G) .
$$

On définit la filtration $V$ par 


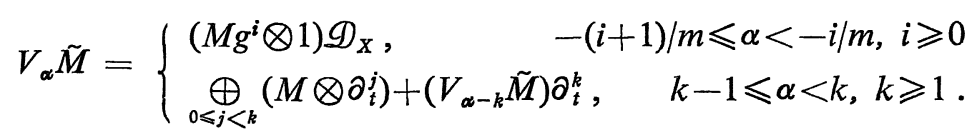

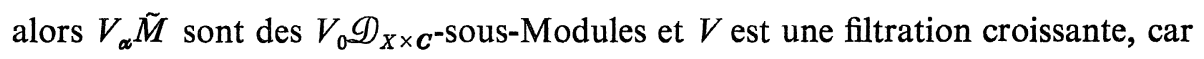

$$
\begin{aligned}
& (u \otimes 1) \partial_{j}=\left(u \partial_{j} \otimes 1\right)-u m g^{m-1}\left(\partial_{j} g\right) \otimes \partial_{t} \\
& \left(u g^{i} \otimes 1\right)(m s+i+1)=\left(u \partial_{j} x_{j} g^{i} \otimes 1\right)-\left(u g^{i} \otimes 1\right) x_{j} \partial_{j}
\end{aligned}
$$

où $s=t \partial_{t}, \partial_{j}=\partial / \partial x_{j}$, et $\left(x_{1}, \cdots, x_{n}\right)$ sont des coordonneés locales de $X$; de plus $V_{\alpha} \tilde{M}$ sont cohérents car ils sont de type fini sur $V_{0} \mathscr{D}_{X \times C}$.

Soient $M\langle D\rangle=M g^{-1}, W$ la filtration canonique de $M\langle D\rangle$, i.e. localement

$$
W_{r} M\langle D\rangle=\left(W_{r} \Omega_{X}^{n}(\log D)\right)^{\text {rang } M}=\sum_{|I| \leqslant r, I \subset\{1, \cdots, l\}} M / x^{I}
$$

si $g=x_{1}, \cdots, x_{l}$ et $n=\operatorname{dim} X$, cf. [6]. On définit la filtration $V$ par

$$
\boldsymbol{V}_{-i / m, k} \tilde{M}=\sum_{r \leqslant \max (2 j+k+1, j)}\left(W_{r} M\langle D\rangle g^{i} \otimes 1\right)(m s+i)^{j} \mathscr{D}_{X}
$$

pour $i>0$, alors ils sont des $V_{0} \mathscr{D}_{X \times C^{-}}$Modules cohérents et $\boldsymbol{V}_{-i, m, k} \tilde{M}=V_{-i, m} \tilde{M}$ pour $k \geqslant l-1, V_{-(i+1) / m} \tilde{M}$ pour $k \leqslant-l$.

Soit $\varepsilon^{k}$ le faisceau d'orientation rel. à $D$ sur $\widetilde{D}^{(k)}$, i.e. localement $\varepsilon^{k}=$ $C D_{1} \wedge \cdots \wedge D_{k}$ sur $\bigcap_{1 \leqslant i \leqslant k} D_{i}$ si $D_{i}$ sont des composants locals de $D$, (voir [6.(3.1.1)]).

On définit les morphismes

$$
\alpha_{i, k}: a_{k *} a_{k}^{!}(M, F)[k] \otimes \varepsilon^{k} \rightarrow G r_{-i / m, k-1}^{V_{1}}(\tilde{M}, F)
$$

comme suit:

D'abord on définit $\alpha_{i k}^{\prime}$ la restriction de $\alpha_{i k}$ sur $\alpha_{k}^{\prime}(M, F)[k] \otimes \varepsilon^{k}=a_{k}^{-1}(M, F)$ $\otimes \omega_{\widetilde{D}^{(k)} / X}\left(\varepsilon^{k}\right)$ par la composition

$$
a_{k}^{-1}(M, F) \otimes \omega_{\widetilde{D}^{(k)} / X}\left(\varepsilon^{k}\right) \hookrightarrow a_{k}^{-1} G r_{k}^{W}\left(M\langle D\rangle g^{i}, F\right) \rightarrow a_{k}^{-1} G r_{-i / m, k-1}^{V}(\tilde{M}, F)
$$

où le premier morphisme est défini par

$$
u \otimes\left(d x_{1} \wedge \cdots \wedge d x_{k}\right)^{-1} \otimes\left(D_{1} \wedge \cdots \wedge D_{k}\right) \mapsto u g^{i} / x_{1} \cdots x_{k},
$$

Ici, $\operatorname{Im} a_{k}=\left\{x_{1}=\cdots=x_{k}=0\right\}$ et $D_{i}=\left\{x_{i}=0\right\}$ localement (rel. à $\tilde{D}^{(k)}$ ).

On définit $\mathscr{H}^{0} a_{k}^{!} M^{\prime}$ pour un $\mathscr{D}_{X}$-Module $M^{\prime}$ par

$$
\mathscr{H}^{0} a_{k}^{!} M^{\prime}=\operatorname{Hom}_{a_{k}^{-1} \mathcal{O}_{X}}\left(\mathcal{O}_{\widetilde{D}^{(k)}}, a_{k}^{-1} M^{\prime}\right) \quad\left(\cup a_{k}^{-1} M^{\prime}\right)
$$

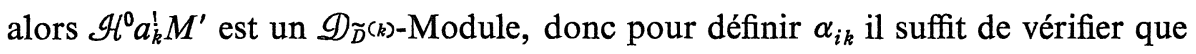
$\operatorname{Im} \alpha_{i k}^{\prime} \subset \mathscr{H}^{0} a_{k}^{\prime} G r_{-i / m, k-1}^{V} \tilde{M}$ et que $\alpha_{i k}^{\prime}$ est $\mathscr{D}_{\tilde{D}^{(k)}}$-linéaire, car localement (rel. à 
$\left.\widetilde{D}^{(k)}\right) a_{k}$ est une immersion fermée et $a_{k *} \mathcal{H}^{0} a_{k}^{!}=\mathcal{H}_{\operatorname{Im} a_{k}}^{0}$ d'après Kashiwara.

Puisque l'assertion est locale, on peut supposer que $\operatorname{Im} a_{k}=\cap D_{i}, D_{i}=$ $\left\{x_{i}=0\right\}$ et $M=\omega_{X}$; alors $\operatorname{Im} \alpha_{i k}^{\prime} \subset \mathcal{H}^{0} a_{k}^{!}$est claire, et on vérifie que $\alpha_{i k}^{\prime}$ est $\mathscr{D}$. linéaire en appliquant (3.6.1.3) à $u=u^{\prime} / x_{1} \cdots x_{k} x_{j}(j>k)$.

3.6.2. Soient $\left(x_{1}, \cdots, x_{n}\right)$ des coordonnées locales de $X$ telles que $g=x_{1} \cdots x_{l}$. Posons $0=\left\{x_{1}=\cdots=x_{n}=0\right\}, \mathcal{O}=\mathcal{O}_{X, 0}, \mathscr{D}=\mathscr{D}_{X, 0}(=\mathcal{O}[\partial])$. On définit pour $I \subset\{1, \cdots, l\}$

$$
\mathcal{O}_{I}=\mathbb{C}\left\{x_{I^{c}}\right\} \subset \mathcal{O}, \quad \text { où } \quad I^{c}=\{1, \cdots, n\} \backslash I .
$$

Soient $\nu \in \mathbb{N}^{l}, I \subset\{1, \cdots, l\}$, on définit

$$
S_{v I}=(x \partial)^{\nu}\left(g / x^{I}\right) \mathcal{O}_{I}\left[\partial_{I}\right] \subset \mathscr{D}
$$

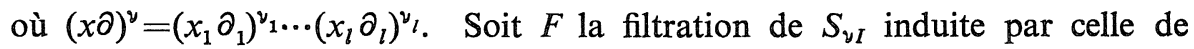
$\mathscr{D}=\mathcal{O}[\partial]$.

Soient $j \in N, I \subset\{1, \cdots, l\}, \mu \in \mathbb{N}^{I}$, on définit

$$
\bar{S}_{I, \mu, j}=\left(g / x^{I}\right)(\partial f)^{\mu} f^{j} \mathcal{O}_{I} \subset \mathcal{O}
$$

où $(\partial f)^{\mu}=T\left(\partial_{j} f\right)^{\mu}{ }_{j}$. Soient $p, q \in \mathbb{N}, k \in \mathbb{Z}$, on définit

$$
\begin{aligned}
& A_{p}=\left\{(I, \mu, j):|\mu|+j=p, \mu \in \mathbb{N}^{I}, I \subset\{1, \cdots, l\}, j \in \mathbb{N}\right\} \\
& A_{p k}^{\prime}=\left\{(I, \mu, j):|I| \leqslant \max (2 j+k+1, j),|\mu|+j=p, \mu \in \mathbb{N}^{l},\right. \\
& I \subset\{1, \cdots, l\}, j \in \mathbb{N}\} \\
& A_{p k}=A_{p} \cap A_{p k}^{\prime} \\
& B_{k}=\left\{(I, \nu):|I| \leqslant \max (2|\nu|+k+1,|\nu|), I \subset\{1, \cdots, l\}, \nu \in \mathbb{N}^{l}\right\} \\
& B_{q k}=\{(I, \nu, j):|I| \leqslant \max (2(|\nu|+j+q)+k+1,|\nu|+j+q), \\
& \left.I \subset\{i, \cdots, l\}, \nu \in \mathbb{N}^{l}, j \in \mathbb{N}\right\}
\end{aligned}
$$

3.6.3. Lemme. $\underset{I \subset\{1, \cdots, l\}, v \in N^{l}}{\bigoplus^{l}}\left(S_{v I}, F\right) \underset{\rightarrow}{\sim}\left(\mathcal{O}\left[\partial^{\prime}\right], F\right)$ où $\partial^{\prime}=\left(\partial_{1}, \cdots, \partial_{l}\right)$.

Preuve. Il suffit de démontrer que $\oplus G r S_{y_{I}} \underset{\mathcal{O}}{\sim}\left[\xi^{\prime}\right]$, où $\xi_{i}=g r \partial_{i}$. Soit $a\left(x, \xi^{\prime}\right)$ $=\sum a_{\mu y} x^{\mu} \xi^{\nu} \in \mathcal{O}\left[\xi^{\prime}\right]$, on définit

$$
\operatorname{supp} a=\left\{(\mu, \nu) \in N^{n+l}: a_{\mu y} \neq 0\right\} .
$$

Soit $I \subset\{1, \cdots, l\}$, on définit

$$
T_{I}=\left\{(\mu, \nu) \in N^{n+l}: \mu_{i}=0 \text { si } i \in I, \mu_{i}>0, \nu_{i}=0 \text { sinon }\right\},
$$

on a alors $a \in G r^{F} S_{\nu_{I}}$ sii supp $a \in(\nu, 0, \nu)+T_{I}$. Puisque $N^{n+l}=\prod_{\nu, I}(\nu, 0, \nu)+T_{I}$, on obtient l'assertion. 
3.6.4. Lemme. $\underset{B_{k}}{\bigoplus} S_{v_{I}}$ est stable par l'action de $\mathscr{D}$ à droite sur $\mathcal{O}\left[\partial^{\prime}\right]=$ $\mathscr{D} / \sum_{j>l} \partial_{j} \mathscr{D}$, i.e.

$$
\bigoplus_{B_{k}} S_{\nu I}=\sum_{B_{k}}(x \partial)^{\nu}\left(g / x^{I}\right) \mathcal{O}\left[\partial^{\prime}\right]
$$

Preuve. Puisqu'on a

$$
\left\{\begin{array}{l}
S_{v_{I}} x_{j} \subset S_{v_{I}} \oplus S_{\nu+1 j, I} \oplus S_{\nu, I \backslash j\}} \\
S_{v_{I}} \partial_{j} \subset S_{v_{I}} \oplus S_{v+1 j, I} \oplus S_{\nu+1 j, I} \cup\{j\}
\end{array}\right.
$$

$\oplus S_{\nu I}$ sont stables par l'action de $C\left[x, \partial^{\prime}\right]$. On vérifie que $F_{p} S_{\nu_{I}}$ sont des sous-espaces fermé de $F_{p} \mathcal{O}\left[\partial^{\prime}\right]$ par la topologie $m$-adique. Puisque $F_{p} \mathcal{O}\left[\partial^{\prime}\right]$ est une somme directe finie de $F_{p} S_{y_{I}}$ et l'action

$$
F_{p} \mathcal{O}\left[\partial^{\prime}\right] \times F_{q} \mathscr{D} \rightarrow F_{p+q} \mathcal{O}\left[\partial^{\prime}\right]
$$

est continue, on obtient l'assertion.

3.6.5. Lemme. Pour $p \in N, k \in Z, \underset{(I, \mu, j) \in A_{p}}{\bigoplus} \bar{S}_{I, \mu, j} \rightarrow \mathcal{O}$ est injectif et

$$
\underset{A_{p k}}{\bigoplus} \bar{S}_{I, \mu, j}=\sum_{A_{p k}^{\prime}}\left(g / x^{I}\right)(\partial f)^{\mu} f^{j} \mathcal{O}, \quad c f . \text { 3.6.2. }
$$

Preuve. Posons $\bar{T}_{I, \mu, j}=\left\{\nu \in \boldsymbol{N}^{n}: \nu_{i}=m(|\mu|+j)-\mu_{i}\right.$ si $i \in I, \nu_{i} \geqslant m(|\mu|+j)+1$ si $i \notin I, i \leqslant l\}$ on a alors $\bar{T}_{I, \mu, j}=\operatorname{supp} \bar{S}_{I, \mu, j}$ et $\underset{(i, \mu, j) \in A_{p}}{\Perp} \bar{T}_{I, \mu, j} \subset \boldsymbol{N}^{n}$; d'où la première assertion. Soit $\lambda_{p}: G r_{p}^{F} \mathcal{O}\left[\partial^{\prime}\right] \rightarrow \mathcal{O}$ le morphisme défini par $\lambda_{p}\left(\partial_{j}\right)=\partial_{j} f$, alors

$$
\bigoplus_{\boldsymbol{A}_{p k}} \bar{S}_{I, \mu, j}=\lambda_{p}\left(G r_{p}^{F}\left(\bigoplus_{B_{k}} S_{\nu_{I}}\right)\right)
$$

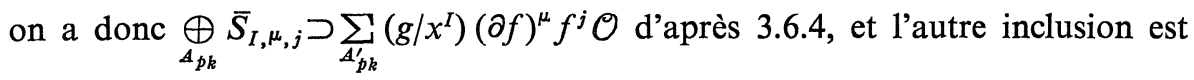
claire; d'où l'assertion.

3.6.6. Supposons maintenant que $M=\omega_{X}, F=G$, cf. 3.6.1. Soit $K^{*}=$ $K\left(\mathcal{O}\left[\partial^{\prime}, s\right]:\left(x_{j} \partial_{j}+(m s+i)\right) \cdot, 1 \leqslant j \leqslant l\right\}[l]$ le complexe de Koszul pour la multiplication à gauche de $\left(x_{j} \partial_{j}+(m s+i)\right) \cdot \operatorname{sur} \mathcal{O}\left[\partial^{\prime}, s\right]$. On définit les filtrations $F$ et $W$ par

$$
\begin{aligned}
& F_{p} K^{-q}=\oplus\left(F_{p-q} \mathcal{O}\left[\partial^{\prime}, s\right]\right) \\
& W_{k} K^{-q}=\oplus\left(\bigoplus_{B_{q_{k}}} S_{\nu I}(m s+i)^{j}\right) .
\end{aligned}
$$

On définit le scindage $W$ par

$$
K_{k}^{-q}= \begin{cases}\bigoplus\left(\bigoplus_{\substack{|I|=2(|\nu|+j+q)+k+1 \\|I|>|\nu|+j+q}} S_{\nu I}(m s+i)^{j}\right) & k \in \boldsymbol{Z} \\ \bigoplus\left(\bigoplus_{|I| \leqslant|\nu|+j+q} S_{\nu I}(m s+i)^{j}\right) & k=-\infty .\end{cases}
$$


Soit $\beta_{i}: K^{0} \rightarrow V_{-i / m} \tilde{M}_{0}\left(\subset \omega_{X}\left[\partial_{t}\right]\right)$ le morphisme $\mathscr{D}[s]$-linéaire défini par $\beta_{i}(P)=$ $(d x \otimes 1) g^{i-1} P$, alors $\beta_{i}$ préserve la filtration $F$.

3.6.7. Lemme. Avec les notations 3.6.6, $(K ; F, W)$ est strict, $H^{j}(K)=0$ pour $j \neq 0$, et on $a$ :

$$
\beta_{i}: H^{0}(K ; F, W) \stackrel{\sim}{\rightarrow}\left(V_{-i / m} \tilde{M}_{0} ; F, V_{-i / m, *}\right),
$$

et

$$
G r_{p}^{F} V_{-i / m, k} \tilde{M}_{0}=\sum_{A_{p k}^{\prime}}\left(g^{i} / x^{I}\right)(\partial f)^{\mu} f^{j} \mathcal{O} \partial_{t}^{p}
$$

Preuve. Puisque $\left\{G r^{F}\left(x_{j} \partial_{j}+m s+i\right)\right\}$ est une suite régulière de $G r^{F} \mathcal{O}\left[\partial^{\prime}, s\right]$, on a $H^{i}\left(G r^{F} K\right)=0$ pour $i \neq 0$; d'où les premières deux assertions, car le scindage de $W$ est compatible avec la différentielle et la filtration $F$. D'après (3.6.1.3) et 3.6.4, on vérifie que $\beta_{i}: H^{0}(K, F, W) \rightarrow\left(V_{-i / m} \tilde{M}_{0}, F, \mathbb{V}\right)$ est bien-défini, et que $\beta_{i} H^{0}\left(W_{k} K\right)=\mathbb{V}_{-i / m, k} \tilde{M}_{0}$; donc il suffit de vérifier que $\beta_{i}: H^{0}(K, F) \rightarrow\left(\mathbb{V}_{-i / m} \tilde{M}_{0}\right.$, $F$ ) est injectif et strict, i.e. $G r^{F} \beta_{i}: H^{0}\left(G r^{F} K\right) \rightarrow G r^{F} \tilde{M}_{0}=\Theta \otimes C\left[g r \partial_{b}\right]$ est injectif. Par la définition de $(K, F)$ on a

$$
H^{0}(K, F) \leftleftarrows \bigoplus_{j, I}\left(S_{0, I}(m s+i)^{j}, F\right)
$$

avec

$$
G r_{p}^{F}\left(S_{0, I}(m s+i)^{j}\right)=\bigoplus_{|\mu|=p-j}\left(g / x^{I}\right) \mathcal{O}_{I} \otimes \partial_{I}^{\mu}(m s+i)^{j} .
$$

Puisque $G r^{F} \beta_{i}$ est défini par la substitution $\partial_{j} \mapsto \partial_{j} f$ et $m s+i \mapsto m f$, on voit que $G r^{F} \beta_{i}$ est injectif d'après 3.6.5. La dernière assertion résulte de 3.6.5, car

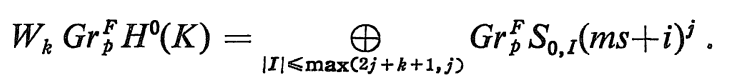

3.6.8. Corollaire. Avec les notations 3.6.7, on a la décomposition comme D-Modules filtrés

$$
G r_{-i / m, k}^{V}\left(\tilde{M}_{0}, F\right)=\bigoplus_{\substack{|I|=2 j+k+1 \\ j \geqslant 0, j+k \geqslant 0}}\left(\mathcal{O}_{I}\left[\partial_{I}\right](m s+i)^{j}, F\right) .
$$

Preuve. D'après (3.6.4.1), on vérifie que chaque $S_{0, I}(m s+i)^{j}$ est stable par l'action de $\mathscr{D}$ sur $G r_{k}^{W} H^{0} K$ pour $|I|=2 j+k+1$, alors l'assertion résulte de 3.6.7, car

$$
G r_{k}^{W} H^{0}(K, F)=\bigoplus_{\substack{|I|=2 j+k+1 \\ j \geqslant 0, j+k \geqslant 0}}\left(S_{0, I}(m s+i)^{j}, F\right) .
$$

3.6.9. Lemme. Avec les notations 3.6.1, les morphismes naturels

$$
\begin{aligned}
\sum_{r \leqslant \max (2 j+k+1, j)}\left(\left(W_{r} M\langle D\rangle g^{i} \cap F_{p-q} M\right) \otimes 1\right)(m s+i)^{j} & F_{q-j} \mathscr{D}_{X} \\
& \rightarrow F_{p} \mathbb{V}_{-i / m, k} G r_{q}^{G} \tilde{M}
\end{aligned}
$$


sont surjectifs, de plus on a les isomorphismes

$$
W_{r} M\langle D\rangle g^{i} \cap F_{p} M=\sum_{|I| \leqslant r} F_{p} M\left(g^{i} / x^{I}\right)
$$

$$
\sum_{\boldsymbol{A}_{q k}^{\prime}} F_{p-q} M\left(g^{i} / x^{I}\right)(\partial f)^{\mu} f^{j}=G r_{q}^{G} F_{p} \boldsymbol{V}_{-i / m, k} \tilde{M}=F_{p} \boldsymbol{V}_{-i / m, k} G r_{q}^{G} \tilde{M}
$$

où $\left(x_{1}, \cdots, x_{n}\right)$ sont des coordonnées locales telles que $g=x_{1} \cdots x_{l}$; en particulier, $(F, G, V)$ sont compatibles.

Preuve. Puisque $F_{p} M$ et $M / F_{p} M$ sont plats sur $\mathcal{O}_{X}$, on a

$$
\begin{gathered}
0 \rightarrow F_{p} M \otimes a \rightarrow M \otimes a \rightarrow M / F_{p} M \otimes a \rightarrow 0 \\
\downarrow \\
0 \longrightarrow F_{p} M \longrightarrow M \longrightarrow M / F_{p} M \longrightarrow 0
\end{gathered}
$$

pour tout idéal $\boldsymbol{a}$ de $\mathcal{O}_{X}$; d'où l'assertion (3.6.9.2) en l'appliquant à $\boldsymbol{a}=$ $\sum_{|I| \leqslant r} \mathcal{O} g^{i} / x^{I}$. On vérifie facilement que l'image du morphisme (3.6.9.1) dans $G r_{q}^{G} \tilde{M}$ $=M$ est le premier terme de (3.6.9.3). D'après 3.6.7, on a

$$
G r_{q}^{G} \boldsymbol{V}_{-i / m, k} \tilde{M}=\sum_{A_{p k}^{\prime}} M\left(g^{i} / x^{I}\right)(\partial f)^{\mu} f^{j},
$$

d'où la surjectivité de (3.6.9.1) en appliquant (3.6.9.4) à

$$
\boldsymbol{a}=\sum_{\boldsymbol{A}_{\boldsymbol{p} k}^{\prime}} \mathcal{O}\left(g^{i} / x^{I}\right)(\partial f)^{\mu} f^{j}
$$

Puisque le morphisme (3.6.9.1) se factorise par l'inclusion naturelle

$$
G r_{q}^{G} F_{p} \boldsymbol{V}_{-i / m, k} \tilde{M} \hookrightarrow F_{p} V_{-i / m, k} G r_{q}^{G} \tilde{M},
$$

on obtient (3.6.9.3); la dernière assertion résulte de 1.2.14.

3.6.10. Proposition. Avec les notations de 3.6.9, $(M, F)$ est quasi-unipotent et régulier le long de $f$, avec la filtration $V$ définie par (3.6.1.1), et on a (3.2.1.3) pour $\alpha=-1$. Pour $i>0$, on $a$

$$
(m s+i)^{k}: G r_{-i / m, k}^{V_{i}}(\tilde{M}, F) \stackrel{\sim}{\rightarrow} r_{-i / m,-k}^{V_{i}}(\tilde{M}, F[-k])
$$

donc la filtration $W$ associée à l'action de $N=s+i / m$ sur $G r_{-i / m}^{V} \tilde{M}$ est induite par $\boldsymbol{V}(c f$. (3.6.1.4)), et on a l'isomorphisme

$$
P_{N} G r_{k-1}^{W} G r_{-i / m}^{V}(\tilde{M}, F) \leftleftarrows a_{k *} a_{k}^{!}(M, F)[k] \otimes \varepsilon^{k} \quad(k>0)
$$

induit par $\alpha_{i k}, c f$. (3.6.1.5).

Preuve. Puisque $\left(\boldsymbol{V}_{-i / m, k} \tilde{M}\right)(m s+i) \subset \boldsymbol{V}_{-i / m, k-2} \tilde{M}$ et $\left(G r_{\alpha-k}^{V} \tilde{M}\right) \partial_{t}^{k}=G r_{\alpha}^{V} \tilde{M}$ pour 
$-1 \leqslant \alpha-k<0$, on vérifie que les conditions de filtration $V$ sont satisfaites, cf. 3.1.1. D'après (3.6.9.3) on a

$$
t: G r_{q}^{G} F_{p} V_{-i / m} \tilde{M} \stackrel{\rightarrow}{\rightarrow} G r_{q}^{G} F_{p} V_{-i / m-1} \tilde{M} \quad(i>0)
$$

donc $t: F_{p} V_{\alpha} \tilde{M} \underset{\rightarrow}{\sim} F_{p} V_{\alpha-1} \tilde{M}$ pour $\alpha<0$. Puisque la décomposition

$$
V_{\alpha} \tilde{M}=\bigoplus_{0 \leqslant j<k}\left(M \otimes \partial_{t}^{j}\right) \oplus\left(V_{\alpha-k} \tilde{M}\right) \partial_{t}^{k}
$$

est compatible avec la filtration $F$, on voit que

$$
\partial_{t}^{k}: F_{p-k} G r_{a-k}^{V} \tilde{M} \rightarrow F_{p} G r_{a}^{V} \tilde{M}
$$

est surjectif pour $-1 \leqslant \alpha-k<0$; d'où la première assertion.

D'après 3.6 .8 , on a

$$
(m s+i)^{k}: G r_{-i / m, k} \tilde{M} \underset{\rightarrow}{\sim} G r_{-i / m,-k}^{V_{M}} \tilde{M}
$$

donc la filtration $W$ est induite par $V$, et la dernière assertion est démontrée pour $M=\omega_{X}, F=G$. En général on a localement

$$
\begin{aligned}
& F_{p} G r_{-i / m, k}^{\boldsymbol{V}_{i / q}} G r_{q}^{G} \tilde{M}=F_{p-q} M \otimes\left(\boldsymbol{a}_{q k} / \boldsymbol{a}_{q, k-1}\right) \\
& =\bigoplus_{\substack{|I|=2 j+k+1 \\
j \geqslant 0, j+k \geqslant 0}} F_{p-q} M \otimes G r_{q-j}^{F}\left(\mathcal{O}_{I}\left[\partial_{I}\right]\right)
\end{aligned}
$$

d'après (3.6.9.3) et 3.6.8, où $\boldsymbol{a}_{q k}=\sum_{\mathbb{q}_{q k}^{\prime}}\left(g^{i} / x^{I}\right)(\partial f)^{\mu} f^{j}$; on a donc

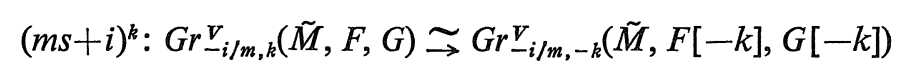

et

$$
G r_{q}^{G} F_{p}\left(P_{N} G r_{-i / m, k}^{V_{i}} \tilde{M}\right)=\bigoplus_{|x|=k+1} F_{p-q} M \otimes G r_{q}^{F}\left(\mathcal{O}_{I}\left[\partial_{I}\right]\right),
$$

i.e. $\alpha_{i, k+1}$ est un isomorphisme filtré, d'où l'assertion.

\section{§4. Structures de Hodge Graduées}

\subsection{Structures de Hodge graduées polarisées}

4.1.1. Soit $M=\oplus M^{i}$ un $C$-module gradué muni de l'action de $l$ telle que $l M^{i} \subset M^{i+2}$. On dit que $M$ est de type $L$ (i.e. de type Lefschetz), si $M^{i}=0$ pour $|i| \gg 0$ et

$$
l^{i}: M^{-i} \stackrel{\sim}{\rightarrow} M^{i} \text { pour tout } i \geqslant 0 .
$$

Si $M$ est de type $L$, on définit la partie primitive par

$$
{ }_{0} M^{-i}=\operatorname{Ker}\left(l^{i+1}: M^{-i} \rightarrow M^{i+2}\right) \text { si } i \geqslant 0 \text {, et } 0 \text { sinon, }
$$


on a alors la décomposition de Lefschetz

$$
M=\bigoplus_{j} M_{j} \quad \text { avec } \quad M_{j}=\bigoplus_{0 \leqslant k \leqslant j} l^{k} M^{-j} .
$$

Notons que, si $\phi: M \rightarrow N$ est un morphisme de modules de type $L, \phi$ préserve la décomposition de Lefschetz, et $\operatorname{Ker} \phi$, Coker $\phi$ et $\operatorname{Im} \phi$ sont de type $L$.

Soient $M$ et $N$ des $\boldsymbol{C}$-modules de type $L$, on dit que $\phi: M \rightarrow N$ est un morphisme de degré 1 , si $\phi$ est $\boldsymbol{C}$-linéaire, $\phi\left(M^{i}\right) \subset N^{i+1}$ et $l \phi=\phi l$.

4.1.2. Lemme. Soit $\phi: M \rightarrow N$ un morphisme de degré 1 de $C$-modules de type $L$, on a alors

$$
\phi\left({ }_{0} M^{-i}\right) \subset{ }_{0} N^{-i+1} \oplus l_{0} N^{-i-1} \quad \text { et } \phi\left(M_{i}\right) \subset N_{i-1} \oplus N_{i+1} .
$$

$\left(\operatorname{Car} \phi\left({ }_{0} M^{-i}\right) \subset \underset{k \geqslant 0}{\bigoplus} N_{i-1+2 k}^{-i+1}\right.$ et $l^{i+1} \phi\left({ }_{0} M^{-i}\right)=0$.)

4.1.3. Lemme. Soit $\phi: M \rightarrow N$ un morphisme de degre 1 , on definit

$$
\operatorname{Im}^{0} \phi=\bigoplus_{j, k} l^{k}\left(\operatorname{Im} \phi \cap_{0} N^{-j}\right),
$$

alors $\operatorname{Im}^{0} \phi$ est de type $L$; de plus, on a

$$
{ }_{0}\left(\operatorname{Im}^{0} \phi\right)^{-j+1}=\operatorname{Im} \phi \bigcap_{0} N^{-j+1}={ }_{0}^{-} \phi_{j}\left({ }_{0}^{+} \phi_{j}^{-1}\left(l_{0}\left(\operatorname{Im}^{0} \phi\right)^{-j-1}\right)\right)
$$

ò̀ $\left.\phi\right|_{M_{j}}={ }^{+} \phi_{j}+{ }^{-} \phi_{j}$ avec ${ }^{ \pm} \phi_{j}\left(M_{j}\right) \subset N_{j \pm 1}$, et ${ }_{0}^{ \pm} \phi_{j}=\left.{ }^{ \pm} \phi_{j}\right|_{0 M^{-j}}$.

Preuve. La première assertion et la première égalité sont claires. Soit $v \in$ $\operatorname{Im} \phi \cap_{0} N^{-j+1}$, on a alors

$$
v=\phi\left(u-u^{\prime}\right) \text { avec } u \in{ }_{0} M^{-j} \text { et } u^{\prime} \in \bigoplus_{k>0} l_{0}^{k} M^{-j-2 k} .
$$

D'après 4.1.1, on a ${ }^{-} \phi_{j} u=v$ et ${ }^{+} \phi_{j} u=\phi u^{\prime} \in l(\operatorname{Im} \phi) \cap N_{j+1}^{-j+1}=l\left(\operatorname{Im} \phi \cap_{0} N^{-j-1}\right)$, car $N_{j+1}^{-j+1}=l_{0} N^{-j-1}$ et $l: N^{-j-1} \rightarrow N^{-j+1}$ est injectif si $j \geqslant 0$. On a donc démontré l'inclusion $\subset$; la réciproque est claire.

4.1.4. Lemme. Soit $i=\min \left\{j \mid M_{j} \neq 0\right\}$, on a alors

$$
\operatorname{Im} \phi \cap N_{i-1}=\left(\operatorname{Im}^{0} \phi\right)_{i-1} \text {. }
$$

Preuve. L'inclusion $\supset$ est claire. Soit $v \in\left(\operatorname{Im} \phi \cap N_{i-1}\right)^{j}$, alors on peut supposer que $j=-i+1+2 k$ avec $0 \leqslant k \leqslant i-1$. Puisque $N_{i-1}^{j}=l_{0}^{k} N^{-i+1}$ et $(\operatorname{Im} \phi)^{j}=$ $l^{k}(\operatorname{Im} \phi)^{-i+1}$ par définition de $i$, on a

$$
\left(\operatorname{Im} \phi \cap N_{i-1}\right)^{j}=l^{k}\left(\operatorname{Im} \phi \cap_{0} N^{-i+1}\right)
$$

car $l^{k}: N^{-i+1} \rightarrow N^{j}$ est injectif; d'où l'assertion. 
4.1.5. Lemme. Soit $\operatorname{Im}^{1} \phi=\operatorname{Im} \phi / \operatorname{Im}^{0} \phi$, alors $\left(\operatorname{Im}^{1} \phi\right)$ [1] est de type $L$, ou $(M[1])^{i}=M^{i+1}$.

Preuve. Par récurrence sur $\left\{i \mid M_{i} \neq 0\right\}$. Si $M=M_{i}$, on a $\operatorname{Im}^{1} \phi=\operatorname{Im} \phi / \operatorname{Im} \phi \bigcap$ $N_{i-1}=\operatorname{Im}^{+} \phi_{i}$, d'après 4.1.4, d'où l'assertion, car $\operatorname{Im}^{+} \phi_{i}=\underset{0 \leqslant k \leqslant i}{\bigoplus} l^{k+} \phi_{i 0} M^{-i}$.

Dans le cas général, on peut supposer que

$$
M=\bigoplus_{k \geqslant 0} M_{i+2 k} \text { et } N=\bigoplus_{k \geqslant 0} N_{i-1+2 k} .
$$

Posons $M^{\prime}=\bigoplus_{k>0} M_{i+2 k}, \phi^{\prime}=\left.\phi\right|_{M^{\prime}}$, on a alors

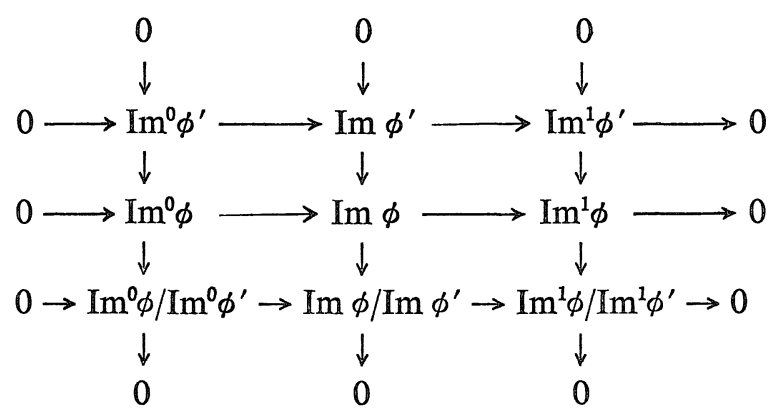

et, par hypothèse de récurrence, il suffit de montrer que $\left(\operatorname{Im}^{1} \phi / \operatorname{Im}^{1} \phi^{\prime}\right)[1]$ est de type $L$.

Soit $\bar{N}=N /\left(\operatorname{Im}^{0} \phi^{\prime}\right)_{i+1}$, soit $\psi: M \stackrel{\phi}{\rightarrow} N \rightarrow \bar{N}$, et soit $\psi^{\prime}=\left.\psi\right|_{M^{\prime}}$, on a alors

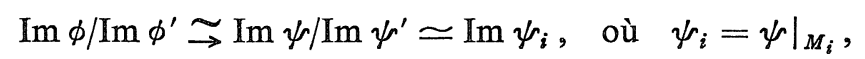

car $\operatorname{Im} \psi^{*}=\operatorname{Im} \phi^{*} /\left(\operatorname{Im}^{0} \phi^{\prime}\right)_{i+1}$ pour $*=\emptyset,^{\prime}$, et $\operatorname{Im} \psi^{\prime} \cap \bar{N}_{i+1}=0\left(\operatorname{car}\left(\operatorname{Im}^{0} \phi^{\prime}\right)_{i+1}=\right.$ $\left.\operatorname{Im} \phi^{\prime} \cap N_{i+1}\right)$. D'après 4.1.3, $\left(\operatorname{Im}^{0} \phi\right)_{j-1}$ ne dépend que de $\left.\phi\right|_{k \leqslant j} ^{\oplus M_{k}}$, en particulier, on a

$$
\left(\operatorname{Im}^{0} \phi\right)_{i+1}=\left(\operatorname{Im}^{0} \phi^{\prime}\right)_{i+1} \text { et }\left(\operatorname{Im}^{0} \psi\right)_{i+1}=\left(\operatorname{Im}^{0} \psi^{\prime}\right)_{i+1}=0
$$

$\operatorname{car}\left(\operatorname{Im}^{0} \psi\right)_{i+1}=\operatorname{Im}\left(\left(\operatorname{Im}^{0} \phi^{\prime}\right)_{i+1} \rightarrow \bar{N}_{i+1}\right)$ d'après 4.1 .3 ; on a alors

$$
{ }_{0}^{+} \phi_{i}^{-1}\left(l_{0}\left(\operatorname{Im}^{0} \phi\right)^{-i-1}\right)={ }_{0}^{+} \psi_{i}^{-1}\left(l_{0}\left(\operatorname{Im}^{0} \psi\right)^{-i-1}\right)=\operatorname{Ker}_{0}^{+} \psi_{i}
$$

et $\operatorname{Im}^{0} \phi / \operatorname{Im}^{0} \phi^{\prime}=\left(\operatorname{Im}^{0} \phi\right)_{i-1}=\left(\operatorname{Im}^{0} \psi\right)_{i-1}=\operatorname{Im}^{0} \psi_{i}$, d'après 4.1.3. On a donc démontré que

$$
\operatorname{Im}^{1} \phi / \operatorname{Im}^{1} \phi^{\prime} \simeq \operatorname{Im}^{1} \psi / \operatorname{Im}^{1} \psi^{\prime} \simeq \operatorname{Im} \psi_{i} / \operatorname{Im}^{0} \psi_{i} \simeq \operatorname{Im}^{1} \psi_{i}
$$

et l'assertion est réduite au cas $M=M_{i}$; d'où l'assertion.

4.1.6. Lemme. Soient $\phi: M \rightarrow N$ et $\psi: N \rightarrow M$ des morphismes de degré 
1 de $\boldsymbol{C}$-modules gradués de type $L$, soient

$$
\langle,\rangle: M \times M \rightarrow C,\langle,\rangle: N \times N \rightarrow C
$$

les dualités telles que $\left\langle M^{i}, M^{j}\right\rangle=\left\langle N^{i}, N^{j}\right\rangle=0$ pour $i+j \neq 0,\langle x, y\rangle= \pm\langle y, x\rangle$ et $\langle l x, y\rangle= \pm\langle x, l y\rangle$ pour $x, y \in M$ ou $N$, et $\langle\phi x, y\rangle= \pm\langle x, \psi y\rangle$ pour $x \in M$, $y \in N$; on a alors

$$
\operatorname{dim}\left(\operatorname{Im}^{0} \phi\right)^{i}=\operatorname{dim}\left(\operatorname{Im}^{1} \psi\right)^{i+1}, \quad \operatorname{dim}\left(\operatorname{Im}^{0} \psi^{1}\right)^{i}=\operatorname{dim}\left(\operatorname{Im}^{1} \phi\right)^{i+1},
$$

si la dimension de $M$ et $N$ est finie.

Preuve. Soient $a_{i}=\operatorname{dim}\left(\operatorname{Im}^{0} \phi\right)^{i}, b_{i}=\operatorname{dim}\left(\operatorname{Im}^{1} \phi\right)^{i+1}, c_{i}=\operatorname{dim}\left(\operatorname{Im}^{0} \psi\right)^{i}$ et $d_{i}=\operatorname{dim}$ $\left(\operatorname{Im}^{1} \psi\right)^{i+1}$, alors $*_{i}=*_{-i}$ pour $*=a, b, c, d$, et

$$
a_{i+1}+b_{i}=c_{-i}+d_{-i-1} \text { pour tout } i,
$$

car rang $\left(\phi: M^{i} \rightarrow N^{i+1}\right)=\operatorname{rang}\left(\psi: N^{-i-1} \rightarrow M^{-i}\right)$ par hypothèse; on a donc

$$
a_{i+1}-d_{i+1}=c_{i}-b_{i}=c_{-i}-b_{-i}=a_{-i+1}-d_{-i+1}=a_{i-1}-d_{i-1} .
$$

Puisque $a_{i}=d_{i}=0$ pour $|i| \gg 0$, on obtient $a_{i}=d_{i}, b_{i}=c_{i}$; d'où l'assertion.

4.1.7. Définition. Soit $M$ un $\boldsymbol{C}$-module gradué de type $L$, on dit que $M$ est de type $H-L$ (Hodge-Lefschetz) de poids $p$, si chaque $M^{i}$ est muni d'une structure de Hodge de poids $p+i$ telle que $l: M^{i} \rightarrow M^{i+2}(1)$ est un morphisme de structures de Hodge. Une polarisation de $M$ est une famille de dualités de structures de Hodge

$$
\langle,\rangle: M^{-i} \times M^{i} \rightarrow \boldsymbol{Q}(-p), \quad i \in \boldsymbol{Z},
$$

telle que $\langle x, y\rangle= \pm\langle y, x\rangle$ et $\langle l x, y\rangle= \pm\langle x, l y\rangle$ et que

$$
\left\langle *, l^{i} *\right\rangle:{ }_{0} M^{-i} \times{ }_{0} M^{-i} \rightarrow \boldsymbol{Q}(-p+i)
$$

soit une polarisation de structure de Hodge à un signe pres, où $\boldsymbol{Q}(p)$ signifie la structure de Hodge de rang un de poids $-2 p$, cf. [6].

Soit $M$ (resp. $N$ ) un module gradué de type $H$ - $L$ de poids $p$ (resp. $p-1$ ), alors un morphisme de degré 1 des modules gradués de type $H$ - $L$ est une famille de morphismes de structures de Hodge

$$
\psi: M^{i} \rightarrow N^{i+1}
$$

compatible avec l'action de $l$. Par hypothèse, $\operatorname{Im}^{0} \phi$ (resp. $\left(\operatorname{Im}^{1} \phi\right)$ [1] est de type $H-L$ de poids $p-1$ (resp. $p$ ). Si $M$ et $N$ sont polarisés, on dit que $\psi$ : $N \rightarrow M(1)$ est dual de $\phi$ si $\langle\phi x, y\rangle= \pm\langle x, \psi y\rangle$ pour $x \in M, y \in N$. 
4.1.8. Lemme. Soient $\phi: M \rightarrow N$ et $\psi: N \rightarrow M(1)$ des morphismes de degré 1 de modules gradués de type $H$-L polarisés. Supposons que $\phi$ et $\psi$ sont duaux l'un à l'autre, alors

$$
{ }^{+} \phi_{j}:\left(\operatorname{Im}^{0} \psi\right)_{j} \rightarrow N_{j+1}(j \geqslant 0) \text { et } \phi: \operatorname{Im}^{0} \psi \rightarrow N
$$

sont injectifs; si, de plus, $(\phi \psi)^{2}=0$ et $j>0$,

$$
{ }_{0}^{-} \phi_{j}: 0\left(\operatorname{Im}^{0} \psi\right)^{-j} \rightarrow N_{j-1}^{-j+1}
$$

est aussi injectif.

Preuve. Soit $v=\psi(u) \in_{0}\left(\operatorname{Im}^{0} \psi\right)^{-j}$. Si $\phi\left(l^{j} v\right)=0$, on a

$$
\left\langle C v, l^{j} \bar{v}\right\rangle= \pm\left\langle C u, \phi\left(l^{j} \bar{v}\right)\right\rangle=0
$$

donc $v=0$ car $v \in{ }_{0} M^{-j}$. Puisque $\phi\left(l^{j} v\right)={ }^{+} \phi_{j}\left(l^{j} v\right)$ et $\left(\operatorname{Im}^{0} \psi\right)_{j}=\bigoplus_{0 \leqslant k \leqslant j} l^{k}{ }_{0}\left(\operatorname{Im}^{0} \psi\right)^{-j}$, on obtient la première assertion. Si $j>0, v \in \in_{0}\left(\operatorname{Im}^{0} \psi\right)^{-j}$ et ${ }_{0}^{-} \phi_{j}(v)=0$, i.e. $\phi(v)=$ ${ }^{+} \phi_{j}(v) \in N_{j+1}^{-j+1}$, alors il existe $w \in{ }_{0} N^{-j-1}$ tel que $\phi(v)=l w$. Puisque $(\phi \psi r)^{2}=0$, on a

$$
\left\langle C w, l^{j+1} \bar{w}\right\rangle= \pm\left\langle C l w, l^{j-1} \overline{l w}\right\rangle= \pm\left\langle C \phi \psi r u, l^{j-1} \phi \psi \bar{u}\right\rangle=0
$$

où $\psi(u)=v$; on a donc $\phi(v)=0$ et $v=0$, d'où la dernière assertion. La deuxième assertion est claire, car $\operatorname{Im}^{0} \psi=\oplus\left(\operatorname{Im}^{0} \psi\right)_{j}, \phi=\oplus\left({ }^{+} \phi_{j}{ }^{-}{ }^{-} \phi_{j}\right)$ et ${ }^{+} \phi_{j}$ : $\left(\operatorname{Im}^{0} \psi\right)_{j} \rightarrow N_{j+1}$ est injectif $(j \geqslant 0)$.

4.1.9. Lemme. Soient $\phi$ et $\psi$ comme dans 4.1.8. Si $\phi \psi \phi=0$, les morphismes

$$
\operatorname{Im}^{0} \phi \stackrel{\psi}{\rightarrow} \operatorname{Im} \psi \phi \rightarrow \operatorname{Im}^{1} \psi \quad \text { et } \quad \operatorname{Im}^{0} \psi \stackrel{\phi}{\rightarrow} \operatorname{Im} \phi \psi \rightarrow \operatorname{Im}^{1} \phi
$$

sont des isomorphismes; en particulier, on a des décompositions

$$
\operatorname{Im} \phi=\operatorname{Im}^{0} \phi \oplus \operatorname{Im} \phi \psi, \quad \operatorname{Im} \psi=\operatorname{Im}^{0} \psi \oplus \operatorname{Im} \psi \phi
$$

telles que

$$
\begin{array}{ll}
\psi: \operatorname{Im}^{0} \phi \stackrel{\operatorname{Im}}{\rightarrow} \phi, \quad & \psi(\operatorname{Im} \phi \psi)=0, \\
\phi: \operatorname{Im}^{0} \psi \stackrel{\longrightarrow}{\rightarrow} \operatorname{Im} \phi \psi, & \phi(\operatorname{Im} \psi \phi)=0 .
\end{array}
$$

Preuve. D'après 4.1.8, on a $\operatorname{Im} \psi \phi \cap \operatorname{Im}^{0} \psi=0$, car $\operatorname{Im} \psi \phi \subset \operatorname{Ker} \phi$; donc $\operatorname{Im} \psi \phi$ $\rightarrow \operatorname{Im}^{1} \psi$ est injectif. De plus, 4.1.8 implique que $\psi: \operatorname{Im}^{0} \phi \rightarrow \operatorname{Im} \psi \phi$ est aussi injectif, alors les isomorphismes

$$
\operatorname{Im}^{0} \phi \stackrel{\psi}{\rightarrow} \operatorname{Im} \psi \phi \underset{\sim}{\rightarrow} \operatorname{Im}^{1} \psi
$$


résultent de 4.1.6.

(De même argument pour l'assertion duale, car $\psi \phi \psi= \pm(\phi \psi \phi)^{*}=0$.)

4.1.10. Lemme. Soient $\phi$ et $\psi$ comme dans 4.1.8. Si $\phi \psi \phi=0, \operatorname{Ker} \phi / \operatorname{Im} \psi \phi$ est de type $H-L$, sur lequel la polarisation de $M$ induit une polarisation (i.e. la partie primitive de $\operatorname{Ker} \phi / \operatorname{Im} \psi \phi$ est représentée par celle de $M$; notons que la dualité induite est bien définie, car $\langle\operatorname{Ker} \phi, \operatorname{Im} \psi\rangle=0$ ).

Preuve. Soient ${ }^{0} N=\operatorname{Im}^{0} \phi,{ }^{1} N=\left({ }^{0} N\right)^{\perp}=\left\{x \in N \mid\langle x, y\rangle=0\right.$ pour tout $\left.y \in{ }^{0} N\right\}$, alors ${ }^{0} N$ et ${ }^{1} N$ sont de type $H-L$ polarisé, et $\operatorname{Im} \phi \psi \subset^{1} N$. Soient $\phi={ }^{0} \phi \oplus^{1} \phi$ avec $\operatorname{Im}{ }^{a} \phi \subset \subset^{a} N(a=0,1)$, et $\psi={ }^{0} \psi \oplus^{1} \psi$ avec ${ }^{a} \psi=\left.\psi\right|_{a_{N}}(a=0,1)$, alors ${ }^{a} \phi$ et ${ }^{a} \psi$ sont duaux l'un à l'autre, et $\operatorname{Ker} \phi=\operatorname{Ker}^{0} \phi \cap \operatorname{Ker}{ }^{1} \phi, \operatorname{Im} \psi \phi=\psi\left(\operatorname{Im}^{0} \phi\right)=\operatorname{Im}\left({ }^{0} \psi\right)$. Puisque

$$
\operatorname{Ker} \phi / \operatorname{Im} \psi \phi=\operatorname{Ker}\left({ }^{1} \phi: \operatorname{Ker}\left({ }^{0} \phi\right) / \operatorname{Im}\left({ }^{0} \psi\right) \rightarrow \operatorname{Im} \phi \psi[1]\right),
$$

on peut remplacer $N, \phi, \psi \operatorname{par}{ }^{0} N,{ }^{0} \phi,{ }^{0} \psi$, $\operatorname{car} \operatorname{Im} \phi \psi[1]$ est de type $H-L$; donc on peut supposer que

$$
\operatorname{Im} \phi=\operatorname{Im}^{0} \phi=N, \quad \operatorname{Im} \psi \phi=\operatorname{Im} \psi \text { et } \phi \psi=0 .
$$

Soient $\phi=\bigoplus_{j} \phi$ avec $\operatorname{Im}_{j} \phi \subset N_{j}$, et $\psi=\bigoplus \psi_{j}$ avec $\psi_{j}=\left.\psi\right|_{N_{j}}$, alors ${ }_{j} \phi$ et $\psi_{j}$ sont duaux l'un à l'autre. Puisque

$\left.\operatorname{Ker} \phi / \operatorname{Im} \psi=\operatorname{Ker}\left({ }_{j} \phi: \operatorname{Ker} \phi^{\prime} / \operatorname{Im} \psi^{\prime} \rightarrow N_{j}\right) / \operatorname{Im}\left(\psi_{j}: N_{j} \rightarrow \operatorname{Ker} \phi^{\prime} / \operatorname{Im} \psi^{\prime}\right)\right)$, où $\phi^{\prime}=\bigoplus_{i \neq j} \phi$ et $\psi^{\prime}=\bigoplus_{i \neq j} \psi_{i}$, on peut supposer que $N=N_{j}$ par récurrence. Soient $\psi={ }^{+} \psi \oplus^{-} \psi$ avec $\operatorname{Im}\left({ }^{ \pm} \psi\right) \subset M_{j \pm 1}$ et ${ }_{0}^{ \pm} \psi=\left.{ }^{ \pm} \psi\right|_{0 N^{-j}}$, soit

$$
{ }^{0} M=\left(\bigoplus_{0 \leqslant k \leqslant j+1} l^{k-1} \operatorname{Im}_{0}^{+} \psi\right) \oplus\left(\bigoplus_{0 \leqslant k \leqslant j} l^{k} \operatorname{Im}-\bar{\psi} \psi\right),
$$

i.e. ${ }^{0} M$ est le plus petit sous-module gradué de type $H$ - $L$ contenant $\operatorname{Im} \psi$; posons ${ }^{1} M=\left({ }^{0} M\right)^{\perp}$, alors $M={ }^{0} M+{ }^{1} M$ et il suffit de montrer que ${ }^{1} M \stackrel{\operatorname{Ker}}{ } \phi / \operatorname{Im} \psi$.

Puisque ${ }^{0} M$ est de type $H-L$ polarisé, on peut supposer que $M={ }^{0} M$, i.e. ${ }^{1} M=0$, alors

$$
+\psi: N \rightarrow \bigoplus_{0 \leqslant k \leqslant j} M_{j+1}^{-j+1+2 k} \text { et } \quad-\psi: \bigoplus_{1 \leqslant k \leqslant j} N^{-j+2 k} \rightarrow M_{j-1}
$$

sont bijectifs d'après 4.1.8; en particulier, on a

$$
\operatorname{dim} M^{-j-1+2 k}= \begin{cases}2 n & \text { si } 0<k<j+1 \\ n & \text { si } k=0 \text { ou } j+1 \\ 0 & \text { sinon }\end{cases}
$$




$$
\operatorname{dim} N^{-j+2 k}= \begin{cases}n & \text { si } 0 \leqslant k \leqslant j \\ 0 & \text { sinon }\end{cases}
$$

où $n=\operatorname{dim} N^{-j}$; on a donc $\operatorname{Ker} \phi / \operatorname{Im} \psi=0$ car $\psi: N^{-j+2 k} \rightarrow M^{-j+1+2 k}$ est injectif et $\phi: M^{-j-1+2 k} \rightarrow N^{-j+2 k}$ est surjectif. Ce qui achève la démonstration.

\subsection{Structure de Hodge bigraduée polarisée}

4.2.1. Soit $L=\bigoplus_{i, j} L_{i}^{j}$ un $\mathbb{C}$-module gradué bigradué. On dit que $L$ est de type $H-L$ de poids $p$, si chaque $L_{i}^{j}$ est muni d'une structure de Hodge de poids $i+j+p$ et si on a des morphismes de structures de Hodge

$$
N: L_{i}^{j} \rightarrow L_{i-2}^{j}(-1), \quad l: L_{i}^{j} \rightarrow L_{i}^{j+2}(1) \quad(i, j \in \mathbb{Z})
$$

tels que $N l=l N, N^{i}: L_{i}^{j} \underset{\rightarrow}{\rightarrow} L_{-i}^{j}(-i)$ pour $i \geqslant 0$ et $l^{j}: L_{i}^{-j} \underset{\rightarrow}{\rightarrow} L_{i}^{j}(j)$ pour $j \geqslant 0$.

Une polarisation de $L$ est une famille de dualités de structures de Hodge

$$
\langle,\rangle: L_{-i}^{-j} \times L_{i}^{j} \rightarrow \mathbb{Q}(-p)
$$

telles que $\langle x, y\rangle= \pm\langle y, x\rangle,\langle l x, y\rangle= \pm\langle x, l y\rangle$ et $\langle N x, y\rangle= \pm\langle x, N y\rangle$ et que

$$
\left\langle *, N^{i} l^{j} *\right\rangle:{ }_{0} L_{i}^{-j} \times{ }_{0} L_{i}^{-j} \rightarrow \boldsymbol{Q}(-p-i+j)
$$

est une polarisation à un signe près pour $i, j \geqslant 0$, où

$$
{ }_{0} L_{i}^{-j}=\operatorname{Ker} N^{i+1} \cap \operatorname{Ker} l^{j+1} .
$$

Une différentielle de $L$ est une famille de morphismes de structures de Hodge

$$
d: L_{i}^{j} \rightarrow L_{i-1}^{j+1}
$$

tels que $d^{2}=0, d l=l d, d N=N d$ et $\langle d x, y\rangle= \pm\langle x, d y\rangle$.

4.2.2. Proposition. Soit $L$ un module bigradue de type $H$-L polarise muni d'une differentielle $d$, alors $\operatorname{Ker} d / \mathrm{Im} d$ est de type $H-L$, sur lequel la polarisation de $L$ induit une polarisation.

Preuve. Posons $C_{i k}^{j}=N^{k}\left(P_{N} L_{i+k}^{j}\right)$ si $i, k \geqslant 0$ et 0 sinon, où $P_{N} L_{i}^{j}=\operatorname{Ker} N^{i+1}$ $\left(\subset L_{i}^{j}\right)$, on a alors

$$
L_{i}^{j}=\bigoplus_{k-k=i} C_{h k}^{j}, \quad \text { et } \quad N^{i-k}: C_{i k}^{j} \underset{\rightarrow}{\rightarrow} C_{k i}^{j}(k-i) \text { si } i \geqslant k .
$$

D'après 4.1.2, on a

$$
d C_{i, 0}^{j} \subset C_{i-1,0}^{j+1} \oplus C_{i, 1}^{j+1} \quad \text { et } \quad d C_{i k}^{j} \subset C_{i-1, k}^{j+1} \oplus C_{i, k+1}^{j+1}
$$

car $d N=N d$. Posons $\left.d\right|_{c_{i k}^{j}}={ }^{+} d+^{-} d$ avec $\operatorname{Im}{ }^{+} d \subset C_{i, k+1}^{j+1}$ et $\operatorname{Im}{ }^{-} d \subset C_{i-1, k}^{j+1}$, on 
a alors des relations ${ }^{+} d^{2}={ }^{-} d^{2}=0,{ }^{+} d^{-} d+^{-} d^{+} d=0$, car $d^{2}=0$. Puisque ${ }^{ \pm} d N=$ $N^{ \pm} d,{ }^{ \pm} d$ est donné par

$$
\gamma=-d: C_{i+1,0}^{j} \rightarrow C_{i, 0}^{j+1} \text { et } \rho=N^{-1} \circ^{+} d: C_{i, 0}^{j} \rightarrow C_{i+1,0}^{j+1}(1)
$$

pour $i \geqslant 0$, où $N^{-1}: C_{i, 1}^{j+1} \underset{\rightarrow}{\rightarrow} C_{i+1,0}^{j+1}(1)$; de plus, $r$ et $\rho$ sont duaux par les dualités

$$
\left\langle *, N^{i} *\right\rangle: C_{i, 0}^{j} \times C_{i, 0}^{-j} \rightarrow \boldsymbol{Q}(-p-i),
$$

car $^{-} d: C_{i+1,0}^{j} \rightarrow C_{i, 0}^{j+1}$ et ${ }^{+} d: C_{0, i}^{-j-1} \rightarrow C_{0, i+1}^{-j}$ sont duaux par les dualités

$$
\langle,\rangle: C_{i, k}^{j} \times C_{k, i}^{-j} \rightarrow \boldsymbol{Q}(-p)
$$

(notons que la dualité est compatible avec la décomposition de Lefschetz rel. à $N$ ).

Posons $C_{i, k}=\bigoplus_{j} C_{i, k}^{j}$ et $C_{i}=C_{i, 0}(i \geqslant 0)$, alors on peut appliquer les résultats de 4.1 à $r: C_{i+1} \rightarrow C_{i}$ et $\rho: C_{i} \rightarrow C_{i+1}(1)$ pour $i \geqslant 0$, car $r \rho r=-r^{2} \rho=0$; en particulier, $\operatorname{Ker} \rho / \operatorname{Im} \gamma \rho$ est de type $H$ - $L$ polarisé d'après 4.1.10.

On définit des sous-modules gradués de $C_{i k}$ pour $i, k \geqslant 0$ par

$$
\begin{aligned}
Z_{i k} & = \begin{cases}N^{k}(\operatorname{Ker} \rho) & \text { si } i=0 \\
N^{k}(\operatorname{Ker} \rho \cap \operatorname{Ker} \gamma) & \text { sinon }\end{cases} \\
B_{i k} & = \begin{cases}N^{k}(\operatorname{Im} \rho) & \text { si } i=0, k>0 \\
N^{k}(\operatorname{Im} \gamma \rho) & \text { sinon }\end{cases}
\end{aligned}
$$

où $\operatorname{Ker} \rho, \operatorname{Im} \rho$, etc. sont des sous-modules de $C_{i+k}$. Posons $H_{i k}=Z_{i k} / B_{i k}$, on a alors

$$
H_{i, 0}=\operatorname{Ker}\left({ }^{1} \gamma:(\operatorname{Ker} \rho / \operatorname{Im} \gamma \rho) \rightarrow(\operatorname{Im} \gamma \rho)[1]\right) \text { pour } i>0,
$$

car $\operatorname{Ker}\left({ }^{0} \gamma\right)=\operatorname{Ker}\left({ }^{0} \rho\right)$, où $\gamma={ }^{0} \gamma+{ }^{1} \gamma, \rho={ }^{0} \rho+{ }^{1} \rho$ avec $\operatorname{Im}\left({ }^{0} \gamma\right)=\operatorname{Im}{ }^{0} \gamma, \operatorname{Im}\left({ }^{1} \gamma\right)=$ $\operatorname{Im} r \rho$ (de même pour $\rho$ ). Puisque (Im $r \rho$ ) [1] est de type $H$ - $L$ d'après 4.1.9, $H_{i, 0}$ est de type $H-L$ polarisé; donc l'assertion résulte du lemme suivant:

4.2.3. Lemme. Posons $H_{i}=\operatorname{Ker}\left(d: L_{i} \rightarrow L_{i-1}\right) / \operatorname{Im}\left(d: L_{i+1} \rightarrow L_{i}\right)$ où $L_{i}=\bigoplus_{j}$ $L_{i}^{j}$, alors les inclusions naturelles $\bigoplus_{i-k=j} H_{i k} \rightarrow H_{j}$ sont des isomorphismes; de plus on $a$

$$
N^{i}: H_{i 0} \stackrel{\sim}{\rightarrow} H_{0 i}(-i) \text { pour } i \geqslant 0,
$$

en particulier, $H_{i 0}$ est la partie primitive de $H_{i}$.

Preuve. Soit $u=\sum u_{k} \in \operatorname{Ker}\left(d: L_{i} \rightarrow L_{i-1}\right)$ avec $u_{k} \in C_{i+k, k}$, posons $v_{k}={ }^{+} d u_{k}=$ ${ }^{-} d u_{k+1} \in C_{i+k, k+1}$. Pour $i+k \geqslant 0, k \geqslant 0$ on a 


$$
v_{k} \in N^{k+1}(\operatorname{Im} \gamma \cap \operatorname{Im} \rho) \subset N^{k+1}(\operatorname{Ker} \gamma \cap \operatorname{Ker} \rho),
$$

donc $v_{k} \in N^{k+1}(\operatorname{Im} \gamma \rho)$ d'après 4.1.9. Soit $w_{k} \in C_{i+2 k+1,0}$ tel que $N^{k+1} \gamma \rho w_{k}=v_{k}$ pour $i+k \geqslant 0, k \geqslant 0$, posons

$$
w=\sum N^{k} w_{k} \in L_{i+1}, \quad u^{\prime}:=u+d w=\sum u_{k}^{\prime}
$$

avec $u_{k}^{\prime} \in C_{i+k, k}$, alors $u_{k}^{\prime}=u_{k}+^{-} d N^{k} w_{k}+^{+} d N^{k-1} w_{k-1}$, donc ${ }^{+} d u_{k}^{\prime}={ }^{+} d u_{k}+$ ${ }^{+} d^{-} d N^{k} w_{k}=v_{k}+N^{k+1} \rho \gamma w_{k}=0$ pour $i+k \geqslant 0, k \geqslant 0$, d'où la surjectivité de $\oplus H_{i+k, k} \rightarrow H_{i}$, car $u_{-1}=0$.

Soit $u=\sum u_{k} \in L_{i+1}$ avec $u_{k} \in C_{i+k+1, k}$, soit $v=d u=\sum v_{k}$ avec $v_{k} \in C_{i+k, k}$, alors $v_{k}=-d u_{k}+^{+} d u_{k-1}$. Supposons que $v_{k} \in Z_{i+k, k}$, alors ${ }^{+} d v_{k}=0$ implique que ${ }^{+} d^{-} d u_{k}=0$, donc ${ }^{-} d u_{k} \in N^{k} \operatorname{Im}$ ro d'après 4.1.9. Si $i+k>0$, on $\mathrm{a}^{+} d u_{k} \in$ $N^{k+1} \operatorname{Im} r \rho$ par le même argument. On a donc

$$
\left(\bigoplus_{k} Z_{i+k, k}\right) \cap \operatorname{Im}\left(d: L_{i+1} \rightarrow L_{i}\right) \subset \bigoplus_{k} B_{i+k, k}
$$

car $^{+} d u_{k-1} \in N^{k} \operatorname{Im} \rho$ et ${ }^{+} d u_{-1}=0$. Puisque la réciproque est claire, on obtient l'injectivité de $\oplus H_{i+k, k} \rightarrow H_{i}$.

Maintenant démontrons:

$$
(\operatorname{Ker} r \cap \operatorname{Ker} \rho) / \operatorname{Im} \gamma \rho \stackrel{\operatorname{Ker} \rho / \operatorname{Im} \rho}{\rightarrow}
$$

où $\operatorname{Ker} r$, etc. sont des sous-modules de $C_{i, 0}(i>0)$.

Soit $u \in(\operatorname{Ker} \gamma \cap \operatorname{Ker} \rho) \cap \operatorname{Im} \rho$, alors $u \in \operatorname{Im} r \rho$ d'après 4.1.9, d'où l'injectivité. Soit $u \in \operatorname{Ker} \rho$. Puisque $r(u) \in \operatorname{Ker} \rho$, on a $\gamma(u) \in \operatorname{Im} r \rho$ d'après 4.1.9, i.e. il existe $v \in C_{i-1,0}$ tel que $r(u)=\gamma \rho(v)$, donc $u-\rho(v) \in \operatorname{Ker} \gamma \cap \operatorname{Ker} \rho$; d'où la surjectivité. Ce qui achève la démonstration de 4.2.3 et de 4.2.2.

4.2.4. Corollaire. Soit ' $L_{i}^{j}=(\operatorname{Im} N)_{i-1}^{j}$, soient

$$
\operatorname{can}=N: L_{i}^{j} \rightarrow{ }^{\prime} L_{i-1}^{j} \quad \text { et } \quad \operatorname{Var}=i d:{ }^{\prime} L_{i}^{j} \hookrightarrow L_{i-1}^{j},
$$

alors

$$
\operatorname{Im}\left(H(\operatorname{can}): H(L) \rightarrow H\left({ }^{\prime} L\right)\right) \oplus \operatorname{Ker}\left(H(\operatorname{Var}): H\left({ }^{\prime} L\right) \rightarrow H(L)\right)=H\left({ }^{\prime} L\right)
$$

ou $H(L)=\operatorname{Ker} d / \operatorname{Im} d$ (de même pour $H\left({ }^{\prime} L\right)$ ).

Preuve. Il faut montrer que $\operatorname{Ker}(H(\operatorname{Var})) \underset{\rightarrow}{\rightarrow} \operatorname{Coker}(H(\operatorname{can}))$. Soit ' $L=\oplus^{\prime} C_{i k}$ la décomposition de Lefschetz comme dans 4.2.2, alors

$$
{ }^{\prime} C_{i k}=C_{i, k+1} \text { si } i, k \geqslant 0 \text { et } 0 \text { sinon, }
$$

en particulier, 4.2 .3 reste valable pour ' $L$, on a donc 


$$
H\left({ }^{\prime} L\right)_{i k}=\left\{\begin{array}{lll}
N^{k+1}((\operatorname{Ker} \gamma \cap \operatorname{Ker} \rho) / \operatorname{Im} \gamma \rho) & \text { si } i>0, k \geqslant 0 \\
N^{k+1}(\operatorname{Ker} \rho / \operatorname{Im} \rho) & \text { si } i=0, k>0 \\
N^{k+1}(\operatorname{Ker} \rho / \operatorname{Im} \gamma \rho) & \text { si } i=k=0 .
\end{array}\right.
$$

Puisque can $\left(C_{i k}\right) \subset^{\prime} C_{i-1, k}$ et $\operatorname{Var}\left({ }^{\prime} C_{i, k}\right) \subset C_{i, k+1}, 4.2 .3$ implique que

Coker $H(\operatorname{can})_{i k}=N(\operatorname{Ker} \rho / \operatorname{Ker} \gamma \cap \operatorname{Ker} \rho)$ si $i=k=0$ et 0 sinon,

Ker $H(\text { Var })_{i k}=N(\operatorname{Im} \rho / \operatorname{Im} r \rho)$ si $i=k=0$ et 0 sinon .

On a donc Ker $H$ (Var) $\underset{\rightarrow}{\rightarrow}$ Coker $H($ can), car

$(\operatorname{Ker} \gamma \cap \operatorname{Ker} \rho) / \operatorname{Im} \gamma \rho \underset{\operatorname{Ker} \rho / \operatorname{Im} \rho}{\sim}$ d'après (4.2.3.1)

d'où l'assertion.

4.2.5. Remarque. Soient $X$ une variété complexe lisse, $S$ un disque ouvert, $f: X \rightarrow S$ un morphisme projectif. Supposons que $f$ est lisse sur $S \backslash\{0\}$ et que $X_{0}=f^{-1}(0)$ est un diviseur réduit à croisements normaux. Steenbrink [29] a construit une suite spectrale pour la filtration par le poids:

$$
E_{1}^{-r, q+r}=\bigoplus_{k \geqslant 0,-r} H^{q-r-2 k}\left(\tilde{X}^{(2 k+r+1)}, \boldsymbol{Q}\right)(-r-k) \Rightarrow H^{q}\left(X_{\infty}, \boldsymbol{Q}\right)
$$

Posons $L_{i}^{j}=E_{1}^{-i, i+j+n}$ où $\operatorname{dim} X=n+1$, alors $L=\bigoplus L_{i}^{j}$ est un module bigradué de type $H-L$ polarisé. Puisque $E_{2}=E_{\infty}, 4.2 .2$ implique que la filtration induite sur $H\left(X_{\infty}, \boldsymbol{C}\right)$ est la filtration de monodromie (à un décalage près). Une démonstration de ce fait est donnée dans [loc. cit.], mais, comme El Zein l'a remarqué, la démonstration de $\tilde{\nu}^{r} \xi=\theta \eta$ (p. 254, $\uparrow l$. 12) n'est pas complète.

Dans [30], Steenbrink généralise ce résultat au cas non réduit, mais la démonstration de 'Lefschetz Vache' pour une $V$-variété projective [loc. cit., (1.13)] n'est pas correcte, car $\pi_{*}[D]=0$ implique seulement $\pi_{*}\left([D] \cap \pi^{*} \eta\right)=0$ (il faut donc utiliser [1. (6.2.10)] ou 5.3.1, $\operatorname{car} \boldsymbol{C}_{X}[\operatorname{dim} X]=\mathcal{I C}(X)$ si $X$ est une $V$ variété.)

Notons que 4.2.2 implique le théorème local des cycles invariants sous la condition que $X_{0}$ est un diviseur à croisements normaux et cohomologiquement de Kähler, i.e. il existe $\omega \in H^{2}\left(X_{0}, \boldsymbol{R}\right)$ dont la restriction à chaque composant de $\tilde{X}_{0}$ est une classe de Kähler (on ne suppose pas que $f$ est projectif, mais seulement que $f$ est propre, cf. [5].)

4.2.6. Remarque. Récemment Deligne m'a donné une nouvelle démonstration de 4.2.2 avec les signes exacts, en utilisant l'analyse harmonique sur les espaces vectoriels de dimension finie [11. II]. Pour l'appliquer à (4.2.5.1), il faut utiliser 5.2.16 pour vérifier les signes. (On ne peut pas utiliser le résultat de Schmid 
avant que la conclusion de 4.2.2 soit démontrée pour (4.2.5.1).)

\section{$\S 5$. Modules de Hodge Polarisables}

\subsection{Modules de Hodge}

5.1.1. Soit $\mathbb{k}$ un sous-corps de $\mathbb{R}$. Soit $M F_{h}\left(\mathscr{D}_{X}\right)$ la catégorie des $\mathscr{D}_{X}$-Modules holonomes munis d'une bonne filtration $F$ (i.e. $G r^{F} M$ sont cohérents sur $G r^{F} \mathscr{D}_{X}$.) Soit $\operatorname{Prev}\left(\mathbb{k}_{X}\right)$ (resp. Perv $\left(\mathbb{C}_{X}\right)$ ) la catégorie des faisceaux pervers définis sur $\mathbb{k}_{X}$ (resp. $C_{X}$ ), cf. [1]. Le foncteur

$$
D R: M F_{h}\left(\mathscr{D}_{X}\right) \rightarrow \operatorname{Perv}\left(\mathbb{C}_{X}\right) \quad\left(\text { resp. } \otimes_{k} \mathbb{C}: \operatorname{Perv}\left(\boldsymbol{k}_{X}\right) \rightarrow \operatorname{Perv}\left(\mathbb{C}_{X}\right)\right)
$$

est exact et fidèle, cf. 2.4.15. Remarque (2). (Dans ce paragraphe la composition Forgeto $D R$ sera notée $D R$, cf. 2.4.11.) On définit

$$
M F_{h}\left(\mathscr{D}_{X}, \mathbb{k}\right)=M F_{h}\left(\mathscr{D}_{X}\right) \underset{\operatorname{Perv}\left(c_{X}\right)}{\times} \operatorname{Perv}\left(\mathbb{k}_{X}\right)
$$

i.e. les objets de $M F_{h}\left(\mathscr{D}_{X}, R_{k}\right)$ sont les couples $((M, F), K) \in M F_{h}\left(\mathscr{D}_{X}\right) \times \operatorname{Perv}\left(\mathbb{k}_{X}\right)$ munis d'un isomorphisme

$$
\alpha: D R(M) \simeq C \otimes_{k} K \text { dans } \operatorname{Perv}\left(\boldsymbol{C}_{X}\right),
$$

et les morphismes sont les couples de morphismes compatibles avec les isomorphismes $\alpha$. Alors les projections naturelles:

$$
M F_{h}\left(\mathscr{D}_{X}, \mathbb{k}\right) \rightarrow M F_{h}\left(\mathscr{D}_{X}\right) \text { et } \quad M F_{h}\left(\mathscr{D}_{X}, \boldsymbol{k}\right) \rightarrow \operatorname{Perv}\left(\mathbb{k}_{X}\right)
$$

sont fidèles. Soit $M G_{h}\left(\mathscr{B}_{X}\right)$ la catégorie des $\mathscr{B}_{X}$-Modules gradués cohérents $M$ 。 tels que $\lim _{\rightarrow} M_{i}$ soient holonomes. On définit similairement

$$
M G_{h}\left(\mathscr{B}_{X}, \boldsymbol{k}\right)=M G_{h}\left(\mathscr{B}_{X}\right) \underset{\operatorname{Perv}\left(\boldsymbol{C}_{X}\right)}{\times} \operatorname{Perv}\left(\boldsymbol{k}_{X}\right),
$$

alors $M G_{h}\left(\mathscr{B}_{X}, k\right)$ est une catégorie abélienne, et $M F_{h}\left(\mathscr{D}_{X}, \mathbb{k}\right)$ s'identifie à la sous-catégorie pleine de $M G_{h}\left(\mathscr{B}_{X}, \mathbb{k}\right)$ des objets $\left(M_{\bullet}, K\right)$ tels que l'action de $1 \in \mathscr{B}_{1}$ sur $M_{\text {. soit injective. }}$

5.1.2. Lemme. $M F_{h}\left(\mathscr{D}_{X}, \mathbb{k}\right)$ est une catégorie exacte, dont une suite courte est exacte sii ses projections dans $M F_{h}\left(\mathscr{D}_{X}\right)$ et $\operatorname{Perv}\left(\boldsymbol{k}_{X}\right)$ sont exactes. De plus Ker, Coker, Im et Coim existent toujours dans $M F_{h}\left(\mathscr{D}_{X}, \mathbb{k}\right)$.

Preuve. La première assertion est claire. La dernière résulte du fait que Ker et Coker existent dans $M F_{h}\left(\mathscr{D}_{X}\right)$ et Perv $\left(\mathbb{k}_{X}\right)$, et sont compatibles avec l'isomorphisme $\alpha$. 
5.1.3. Soient $(M, F, K) \in M F_{h}\left(\mathscr{D}_{X}, \boldsymbol{k}\right)$ et $n \in \mathbb{Z}$. On définit:

$$
(M, F, K)(n):=(M(n), F[n], K(n)), \mathrm{cf} .(2.0 .2) .
$$

Soit $f: X \rightarrow Y$ un morphisme propre. Soit $(M, F, K) \in M F_{h}\left(\mathscr{D}_{X}, \boldsymbol{k}\right)$. Si $f_{*}(M, F)($ cf. 2.3$)$ est strict, on définit:

$$
\mathscr{H}^{i} f_{*}(M, F, K)=\left(\mathcal{H}^{i} f_{*}(M, F),{ }^{p} \mathcal{H}^{i} f_{*} K\right)
$$

muni de l'isomorphisme: $D R_{Y} \mathcal{H}^{i} f_{*}(M, F)={ }^{p} \mathscr{H}^{i} f_{*} D R_{X}(M, F) \stackrel{\alpha}{\simeq}{ }^{p} \mathcal{H}^{i} f_{*} K \otimes_{k} \boldsymbol{C}$. Notons que $f_{*}(M, F)$ est un Module filtré, si $f$ est une immersion fermée.

Soit $g$ une fonction holomorphe sur $X$, posons $(\tilde{M}, F)=i_{g *}(M, F)$ comme dans 3.2. Si $(M, F)$ est quasi-unipotent et régulier le long de $g$ (cf. 3.2), on définit:

$$
\begin{aligned}
& \psi_{g}(M, F, K)=\left(\underset{-1 \leqslant \infty_{<}<0}{\bigoplus_{\alpha}}\left(G r_{\alpha}^{V} \tilde{M}, F[1]\right),{ }^{p} \psi_{g} K\right) \\
& \phi_{g, 1}(M, F, K)=\left(\left(G r_{0}^{V} \tilde{M}, F\right),{ }^{p} \phi_{g, 1} K\right)
\end{aligned}
$$

où ${ }^{p} \psi_{g} K:=\left(\psi_{g} K\right)[-1]$ (de même pour $\left.{ }^{p} \phi_{g, 1}\right)$, cf. (5.2.1.1). Ici, on utilise la proposition 3.4.12 pour définir l'isomorphisme $\alpha$. De plus on a les morphismes

$$
N: \psi \rightarrow \psi(-1) \quad\left(\text { de même pour } \phi_{1}\right)
$$

$$
\text { can: } \psi_{1} \rightarrow \phi_{1} \text { et } \operatorname{Var}: \phi_{1} \rightarrow \psi_{1}(-1)
$$

par la même proposition, où $\psi=\psi_{g}(M, F, K)$, etc. Ici, on utilise 3.4 .14 pour la définition de Var: ${ }^{p} \phi_{g, 1} K \rightarrow{ }^{p} \psi_{g, 1} K$, etc.

Soit $(M, F, K) \in M F_{h}\left(\mathscr{D}_{X}, \boldsymbol{k}\right)$. On dit que $(M, F, K)$ est à support strict $Z$, si supp $M=Z$, et si $(M, F, K)$ n'a ni un sous-objet, ni un objet quotient, dont le support est plus petit que $Z$. On dit que $(M, F, K)$ admet la décomposition par support strict, si on a une décomposition (localement finie) dans $M F_{h}\left(\mathscr{D}_{X}, \boldsymbol{k}\right)$ :

$$
(M, F, K)=\oplus_{Z}\left(M_{Z}, F, K_{Z}\right)
$$

telle que $\left(M_{Z}, F, K_{Z}\right)$ est à support strict $Z$. Alors cette décomposition est unique, i.e. s'il y a deux isomorphismes $u_{i}:(M, F, K) \rightarrow \oplus_{Z}\left(M_{Z}^{i}, F, K_{Z}^{i}\right)$ $(i=1,2)$, il existe des uniques isomorphismes $v_{Z}:\left(M_{Z}^{1}, F, K_{Z}^{1}\right) \rightarrow\left(M_{Z}^{2}, F, K_{Z}^{2}\right)$ tels que $u_{2}=\left(\oplus v_{Z}\right) u_{1}$. On dit que $\left(M_{Z}, F, K_{Z}\right)$ est la $Z$-composante de $(M, F, K)$.

5.1.4. Lemme. Soient $(M, F, K) \in M F_{h}\left(\mathscr{D}_{X}, \boldsymbol{k}\right)$ et $\mathrm{g}$ une fonction holomorphe sur $X$. Supposons que $(M, F)$ soit quasi-unipotent et régulier le long de $g$ 
(cf. 3.2), on a alors

$$
\psi_{g}(M, F, K), \phi_{g, 1}(M, F, K) \in M F_{h}\left(\mathscr{D}_{X}, \mathbb{R}\right),
$$

et $K,(M, F)$ et $(M, F, K)$ n'ont pas de sous-objets (resp. objets quotients) à supports dans $g^{-1}(0)$ sii can: $\psi_{1} \rightarrow \phi_{1}$ est surjectif (resp. Var: $\phi_{1} \rightarrow \psi_{1}(-1)$ est injectif): où $\psi_{1}=\psi_{g, 1}(M, F, K)$, etc.; de plus les conditions suivantes sont équivalentes:

i) On a une décomposition dans $M F_{h}\left(\mathscr{D}_{X}, \mathbb{k}\right)$ :

$$
\phi_{1}=\operatorname{Ker}\left(\operatorname{Var}: \phi_{1} \rightarrow \psi_{1}(-1)\right) \oplus \operatorname{Im}\left(\operatorname{can}: \psi_{1} \rightarrow \phi_{1}\right) \text {. }
$$

ii) On a une (unique) décomposition dans $M F_{h}\left(\mathscr{D}_{X}, \mathbb{R}\right)$ :

$$
(M, F, K)=\left(M_{1}, F, K_{1}\right) \oplus\left(M_{2}, F, K_{2}\right)
$$

telle que supp $M_{2} \subset g^{-1}(0)$ et que $\left(M_{1}, F, K_{1}\right)$ n'a ni un sous-objet, ni un objet quotient, à support dans $g^{-1}(0)$.

Preuve. Posons $(\tilde{M}, F, \widetilde{K})=i_{g *}(M, F, K)$, alors l'assertion (5.1.4.1) résulte de la définition 3.2.1 et de la preuve de 3.4.9. Puisque les sous-objets de $(M, F, K)$ (i.e. les monomorphismes stricts dans $(M, F, K)$ ) correspondent à ceux de $(M, K)$ dans $M_{h}\left(\mathscr{D}_{X}\right) \underset{\operatorname{Perv}\left(C_{X}\right)}{\times} \operatorname{Perv}\left(\mathbb{k}_{X}\right)$ (de même pour $(M, F)$ ), la deuxième assertion est réduite à celle pour $\tilde{K}, \tilde{M}$ et $(\tilde{K}, \tilde{M})$. On définit:

$$
\tilde{M}_{1}=\left(V_{<0} \tilde{M}\right) \mathscr{D}_{X \times C} \quad \text { et } \quad \tilde{M}_{2}=i_{*}\left(\operatorname{Ker} t: G r_{0}^{V} \tilde{M} \rightarrow G r_{-1}^{V} \tilde{M}\right) \text {, }
$$

où $i: X=X \times\{0\} \rightarrow X \times \mathbb{C}$, alors $\tilde{M} / \tilde{M}_{1}$ (resp. $\tilde{M}_{2}$ ) est le plus grand objet quotient (resp. sous-objet) de $\tilde{M}$ à support dans $X \times\{0\}$ d'après 3.1.8. Soit $\tilde{K} / \widetilde{K}_{1}$ (resp. $\widetilde{K}_{2}$ ) le plus grand objet quotient (resp. sous-objet) de $\tilde{K}$ à support dans $X \times\{0\}$. Par la théorie de prolongement de faisceaux pervers de DeligneMacPherson-Verdier (voir, par exemple, [34]), $\widetilde{K}_{1}^{i}$ (resp. $\widetilde{K}_{2}$ ) correspond au diagramme:

$$
\left(\left.\tilde{K}\right|_{X \times C^{*}},\left(\psi_{1} \widetilde{K} \rightleftarrows \operatorname{Im} \text { can }\right)\right) \quad(\text { resp. }(0,(0, \rightleftarrows \operatorname{Ker} \text { Var })))
$$

(de même pour $\tilde{K} \otimes C$ ); alors l'isomorphisme $\alpha$ induit

$$
D R\left(\tilde{M}_{1}\right) \simeq \tilde{K}_{1} \otimes C \quad \text { et } \quad D R\left(\tilde{M}_{2}\right) \simeq \widetilde{K}_{2} \otimes C
$$

d'après 3.4.12, car $\partial_{t}: G r_{-1}^{V} \tilde{M}_{1} \rightarrow G r_{0}^{V} \tilde{M}_{1}$ est surjectif par le même argument que dans la preuve de 3.2.2. (ii); donc $(\tilde{M}, \tilde{K}) /\left(\tilde{M}_{1}, \widetilde{K}_{1}\right)$ (resp. $\left.\left(\tilde{M}_{2}, \widetilde{K}_{2}\right)\right)$ est le plus grand objet quotient (resp. sous-objet) à support dans $X \times\{0\}$; d'où la deuxième assertion (car $D R$ et $\otimes \mathbb{C}$ sont exacts et fidèles, cf. 5.1.1). Prouvons 
la dernière assertion. L'implication: ii) $\Rightarrow$ i) est claire. Si (5.1.4.2) est vérifiée, on a la décomposition:

$$
(\tilde{M}, \tilde{K})=\left(\tilde{M}_{1}, \tilde{K}_{1}\right) \oplus\left(\tilde{M}_{2}, \tilde{K}_{2}\right)
$$

par l'argument ci-dessus, donc il suffit de montrer que cette décomposition est compatible avec la filtration $F$. D'après 3.2.2, l'assertion est réduite à la décomposition:

$$
\left(V_{0} \tilde{M}, F\right)=\left(V_{0} \tilde{M}_{1}, F\right) \oplus\left(V_{0} \tilde{M}_{2}, F\right)
$$

Soit $u \in F_{p} V_{0} \tilde{M}$, on a alors $u=u_{1}+u_{2}$ avec $u_{i} \in V_{0} M_{i}$ d'après 3.1.6; d'où $G r_{0}^{V} u_{\imath} \in F_{p} G r_{0}^{V} \tilde{M} \cap G r_{0}^{V} \tilde{M}_{i}$ par la condition (5.1.4.2); donc il suffit de vérifier que l'isomorphisme dans la preuve de 3.1.8:

$$
\operatorname{Ker}(t: \tilde{M} \rightarrow \tilde{M}) \rightarrow \operatorname{Ker}\left(t: G r_{0}^{V} \tilde{M} \rightarrow G r_{-1}^{V} \tilde{M}\right)
$$

induit un isomorphisme filtré; mais cela résulte de la condition (3.2.1.2). Ce qui achève la démonstration.

5.1.5. Corollaire. Soit $(M, F, K) \in M F_{h}\left(\mathscr{D}_{X}, \boldsymbol{k}\right)$. Supposons que la condition suivante soit satisfaite:

(5.1.5.1) $(M, F)$ est quasi-unipotent et régulier le long de $g$ pour toute fonction holomorphe $g$ localement définie sur $X$.

Alors les conditions suivantes sont équivalentes:

(5.1.5.2) la condition (5.1.4.2) est satisfaite pour toute g comme ci-dessus,

(5.1.5.3) la restriction de $(M, F, K)$ à tout ouvert de $X$ admet la décomposition par support strict, paramétrée par des sous-ensembles fermés analytiques irréductibles.

Preuve. Puisque la décomposition est unique, la condition (5.1.5.3) est locale, alors l'équivalence résulte du lemme ci-dessus.

5.1.6. Soit $M F_{h}\left(\mathscr{D}_{X}, \boldsymbol{k}\right)_{(0)}$ la sous-catégorie pleine de $M F\left(\mathscr{D}_{X}, \boldsymbol{k}\right)$ des objets satisfaisant les conditions (5.1.5.1-3). On définit $M H(X, \boldsymbol{k}, n)$ les catégories des Modules de Hodge poids $n$, comme la famille (paramétrée par $X$ et $n$ ) des plus grandes sous-catégories pleines de $M F_{h}\left(\mathscr{D}_{X}, \boldsymbol{k}\right)_{(0)}$ satisfaisant les conditions:

(5.1.6.1) $\mathrm{Si}(M, F, K) \in M H(X, \boldsymbol{k}, n)$ et si supp $M=\{x\}$, il existe $\left(H_{\boldsymbol{C}}, F, H_{k}\right)$ une $\boldsymbol{k}$-structure de Hodge de poids $n$ (cf. [6]) telle que $i_{x *}\left(H_{C}, F, H_{k}\right)$ $\simeq(M, F, K)$, cf. (5.1.3.2), où $i_{x}:\{x\} \hookrightarrow X$. 
(5.1.6.2) Si $(M, F, K) \in M H(X, \mathbb{R}, n)$ et si $g$ est une fonction holomorphe sur un ouvert $U$ de $X$, on a:

$G r_{i}^{W} \psi_{g}(M, F, K), G r_{i}^{W} \phi_{g, 1}(M, F, K) \in M H(U, \mathbb{k}, i)$.

Ici $W$ est la filtration de $\psi_{g}(M, K)\left(\operatorname{resp} . \phi_{g, 1}(M, K)\right)$ associée à l'action de $N$ (cf. (5.1.3.4)) décalée par $n-1$ (resp. $n$ ), i.e.

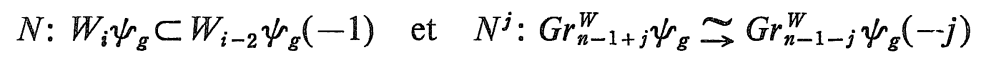

(de même pour $\phi_{g, 1}$, en remplaçant $n-1$ par $n$ ), cf. 1.3.9.

En fait, soit $M F_{h}\left(\mathscr{D}_{X}, \mathbb{k}\right)_{(j)} \quad(j>0)$ la sous-catégorie pleine de $M F_{h}\left(\mathscr{D}_{X}, \not{k}\right)_{(j-1)}$ définie par:

$(M, F, K) \in M F_{h}\left(\mathscr{D}_{X}, \mathbb{R}\right)_{(j)}$ sii $G r_{i}^{W} \psi_{g}(M, F, K), G r_{i}^{W} \phi_{g, 1}(M, F, K) \in$ $M F_{h}\left(\mathscr{D}_{U}, \mathbb{k}\right)_{(j-1)}$ pour toute $g$ comme dans (5.1.6.2),

et soit $M F_{h}\left(\mathscr{D}_{X}, \mathbb{R}\right)_{(\infty)}=\bigcap_{j} M F_{h}\left(\mathscr{D}_{X}, \mathbb{R}\right)_{(j)}$; alors $\operatorname{MH}(X, \mathbb{R}, n)$ est la souscatégorie pleine de $M F_{h}\left(\mathscr{D}_{X}, \mathbb{R}\right)_{(\infty)}$ des objets $(M, F, K)$ satisfaisant la condition:

Pour $g_{1}, \cdots, g_{r}$ des fonctions holomorphes sur un ouvert $U$ de $X$ telles que $\bigcap_{j} g_{j}^{-1}(0)=\{x\}, G r_{j_{r}}^{W} \Psi_{g_{r}}^{\nu_{r}} \cdots G r_{j_{1}}^{W} \Psi_{g_{1}}^{\nu_{1}}(M, F, K)$ est l'image directe d'une $\mathbb{k}$-structure de Hodge de poids $j_{r}$ pour tout $\nu \in\{0,-1\}^{r}$, où $\Psi_{g_{i}}^{v_{i}}=\psi_{g i}\left(\right.$ si $\left.\nu_{i}=-1\right)$ et $\phi_{g_{i, 1}}$ (sinon).

Ici, la filtration $W$ de $\Psi_{g_{i}}^{\nu_{i}} G r_{j_{i-1}}^{W} \Psi_{g_{i-1}}^{\nu_{i-1}} \ldots G r_{j_{1}}^{W} \Psi_{g_{1}}^{\nu_{1}}(M, K)$ est décalée par $j_{i-1}+\nu_{i}$, où $j_{0}=n$.

On dit que $(M, F, K) \in M H(X, \mathbb{k}, n)$ est un Module de Hodge de poids $n$ à support strict $Z$, si $(M, F, K)$ est à support strict $Z$, cf. 5.1.3. Soit $M H_{Z}(X, \mathbb{k}, n)$ la sous-catégorie pleine de tels Modules, on a alors la décomposition:

$$
M H(X, \mathbb{R}, n)=\oplus_{Z} M H_{Z}(X, \mathbb{k}, n)
$$

paramétrée par les sous-espaces analytiques fermés irréductibles, d'après 5.1.5 et par le lemme suivant:

5.1.7. Lemme. La condition de Module de Hodge est locale, et stable par facteur direct dans $M F_{h}\left(\mathscr{D}_{X}, \mathbb{k}\right)$.

Preuve. On vérifie que les conditions (5.1.5.1-2) sont locales et stables par facteur direct, alors la condition de $M F_{h}\left(\mathscr{D}_{X}, \mathbb{k}\right)_{(j)}$ est local et stable par facteur direct dans $M F_{h}\left(\mathscr{D}_{X}, \mathbb{k}\right)$, donc l'assertion résulte du fait que les structures de 
Hodge sont stables par facteur direct dans $M F_{h}(\boldsymbol{C}, \boldsymbol{k})$, où $\boldsymbol{C}=\mathscr{D}_{p t}$.

5.1.8. Lemme. Soit $(M, F, K) \in M F_{h}\left(\mathscr{D}_{X}, k\right)_{(0)}, \quad c f$. 5.1.6. Alors $(M, F, K) \in M H(X, k, n)$ sii la condition suivante est vérifiée:

Pour tout ouvert $U$ de $X$, pour toute fonction holomorphe $g$ sur $U$, et pour tout sous-espace analytique fermé irréductible $Z$ de $U$ tel que $g^{-1}(0) \downarrow Z$ ou $\operatorname{dim} Z=0$, on $a$

$$
G r_{i}^{W} \psi_{g}\left(M_{Z}, F, K_{Z}\right), G r_{i}^{W} \phi_{g, 1}\left(M_{Z}, F, K_{Z}\right) \in M H(U, k, i),
$$

où $\left(M_{Z}, F, K_{Z}\right)$ est la Z-composante de $\left.(M, F, K)\right|_{U}, c f$. (5.1.3.5).

Preuve. Si supp $M \subset g^{-1}(0)$, on a

$$
\psi_{g}(M, F, K)=0, \quad \phi_{g, 1}(M, F, K)=(M, F, K)
$$

et l'action de $N$ sur $\phi_{g, 1}$ est nulle d'après 3.2.6; donc l'assertion résulte par récurrence sur $\operatorname{dim} \operatorname{supp} M$.

5.1.9. Lemme. Soit $i: X \hookrightarrow Y$ une immersion fermée, on a alors une équivalence de catégories:

$$
i_{*}: M H_{Z}(X, \boldsymbol{k}, n) \rightarrow M H_{Z}(Y, k, n)
$$

pour $Z$ une sous-espace analytique fermée irréductible de $X$; en particulier, $M H_{\{x\}}(X, k, n)$ est équivalente à la catégorie des $\boldsymbol{k}$-structures de Hodge de poids $n$.

Preuve. D'après 5.1.7, on peut supposer que $\operatorname{codim}_{Y} X=1$, alors pour $\left(M^{\prime}, F, K^{\prime}\right) \in M F_{h}\left(\mathscr{D}_{Y}, \not k\right)_{(0)}$ (cf. 5.1.6), il existe $(M, F, K) \in M F_{h}\left(\mathscr{D}_{X}, k\right)$ tel que $i_{*}(M, F, K)=\left(M^{\prime}, F, K^{\prime}\right)$ d'après 3.2.2. (iii) et 3.2.5, donc il suffit de vérifier que pour $(M, F, K) \in M F_{h}\left(\mathscr{D}_{X}, \mathbb{k}\right)$, on a:

$$
(M, F, K) \in M H_{Z}(X, k, n) \quad \text { sii } \quad i_{*}(M, F, K) \in M H_{Z}(Y, k, n) .
$$

Soit $g$ une fonction holomorphe localement définie sur $Y$, posons

$$
h=g i, \quad(\tilde{M}, F)=i_{h *}(M, F) \quad \text { et } \quad\left(\tilde{M}^{\prime}, F\right)=i_{g *} i_{*}(M, F),
$$

cf. 3.2, alors $\left(\tilde{M}^{\prime}, F\right)=(i \times i d)_{*}(\tilde{M}, F)$ où $i \times i d: X \times C \rightarrow Y \times C$. Puisque pour toute $h$ sur $X$, il existe (localement) $g$ sur $Y$ telle que $h=g i$, l'assertion résulte de 5.1.8 par récurrence sur $\operatorname{dim} \operatorname{supp} M$.

5.1.10. Lemme. Soit $(M, F, K) \in M H_{Z}(X, \boldsymbol{k}, n)$, alors $K$ est un complexe d'intersection $\mathcal{G C}_{Z}(L)$ et $(M, F, K)$ est génériquement une variation de structures 
de Hodge de poids $n-d_{Z}$, i.e. pour $U$ un ouvert de Zariski lisse de $Z$ sur lequel $K\left[-d_{z}\right]$ est un système local $L$, il existe une variation de structures de Hodge $\left(L \otimes \Theta_{U}, F, L\right)$ de poids $n-d_{z}$ telle que

$$
\left.(M, F, K)\right|_{X \backslash(z \backslash U)} \simeq i_{*}\left(L \otimes \Omega_{U}^{d} U, F, L\right)
$$

où $i: U \rightarrow X \backslash(Z \backslash U)$. Ici la filtration $F$ de $L \otimes \Omega_{U}^{d} U$ est définie par $F_{p}\left(L \otimes \Omega_{U}^{d} U\right)$ $=\Omega_{U}^{d} \sigma \otimes_{\mathcal{O}} F^{-p-d_{Z}}\left(L \otimes \mathcal{O}_{U}\right)$.

Preuve. La première assertion résulte de 5.1.4. Pour la dernière assertion, on peut supposer que $U=X$ d'après 5.1.9. On voit que $G r_{p}^{F} M$ sont des $\mathcal{O}_{X}$-Modules localement libres de type fini et que $G r_{p}^{F} M=0$ pour $p \gg 0$ (localement sur $X$ ), par récurrence sur $d_{X}$, en utilisant 3.2.5 et 3.2.7. En fait $M$ est cohérent (donc libre) sur $\mathcal{O}_{X}$ d'après $\left[19\right.$, Th. 10.1.1], alors la filtration $V$ le long de $X_{0}=\left\{x_{1}=0\right\}$ est la filtration $x_{1}$-adique, où $x_{1}$ est une coordonnée locale de $X$; donc $i_{*}(i *(M, F)[-1], i * K[-1]) \simeq \psi_{x_{1}}(M, F, K)$ (cf. 3.5.1 et 3.5.9 pour la définition de $\left.i^{*}\right)$, où $i: X_{0} \rightarrow X$; d'où l'assertion.

5.1.11. Corollaire。 $\operatorname{Hom}\left(M H_{Z}(X, \mathbb{k}, n), M H_{Z^{\prime}}\left(X, \mathbb{k}, n^{\prime}\right)\right)=0$ si $Z \neq Z^{\prime}$ ou $n>n^{\prime}$.

(C'est clair.)

5.1.12. Lemme. Soit $M$ un objet d'une catégorie abélienne, muni d'un endomorphisme nilpotent $N$. Posons $M^{\prime}=\operatorname{Coim} N$. Soient $u: M \rightarrow M^{\prime}$ et $v: M^{\prime} \rightarrow M$ les morphismes naturels tels que $v u=N$ et $u v=N$, soit $W$ la filtration de $M$ et de $M^{\prime}$ associée à $N$, cf. 1.3.9. Alors $u W_{i} \subset W_{i-1}, v W_{i} \subset W_{i-1}$ et

$$
u:(M, W) \rightarrow\left(M^{\prime}, W[1]\right) \text { et } \quad v:\left(M^{\prime}, W\right) \rightarrow(M, W[1])
$$

sont stricts.

Preuve. D'après [7, (1.6.5)], le morphisme $N:(M, W) \rightarrow(M, W[2])$ est strict. On définit $\left(M^{\prime}, W^{\prime}\right)=\operatorname{Coim}\left(N:(M, W[-1]) \rightarrow(M, W[1])\right.$, alors $G r_{i}^{W^{\prime}} M^{\prime}=$ $\operatorname{Coim}\left(N: G r_{i+1}^{W} M \rightarrow G r_{i-1}^{W} M\right)$, donc $W^{\prime}$ est la filtration associée à $N$ par la décomposition de Lefschetz de $G r^{W} M$; d'où l'assertion.

5.1.13. Lemme. Soit $(M, F, K) \in M H_{Z}(X, k, n)$, alors $G r^{F} M$ est un $G r^{F} \mathscr{D}_{X}$-Module de Cohen-Macaulay, i.e. $\mathbb{D}(M, F)$ est strict.

Preuve. Par récurrence $\operatorname{sur} \operatorname{dim} Z$. Si $\operatorname{dim} Z=0$, l'assertion est claire. Supposons que $\operatorname{dim} Z>0$. Pour toute fonction (locale) $g$ telle que $\operatorname{dim} Z \cap g^{-1}(0)$ $<\operatorname{dim} Z$, posons $(\tilde{M}, F)=i_{g *}(\tilde{M}, F)$, alors il suffit de vérifier que $G r^{F} \tilde{M}_{(x, 0)}$ est 
de Cohen-Macaulay sur $G r^{F} \mathscr{D}_{X \times C,(x, 0)}$ pour tout $x \in g^{-1}(0)$. Soit $(L \cdot F, V) \rightarrow$ $(\tilde{M}, F, V)_{(x, 0)}$ une résolution par des modules induits libres de type fini, i.e. chaque $\left(L^{i}, F, V\right)$ est une somme directe finie de $(\mathscr{D}, F[p], V[\alpha])$ avec $p \in \mathbb{Z}$, $-1 \leqslant \alpha \leqslant 0$ où $\mathscr{D}=\mathscr{D}_{X \times \boldsymbol{C},(x, 0)}$. On définit $\mathbb{D}(\tilde{M}, F, V)_{(x, 0)}=\boldsymbol{D}\left(L^{\bullet}, F, V\right)$ par:

$$
\boldsymbol{D}(\mathscr{D}, F[p], V[\alpha])=(\omega \otimes \mathscr{D}, F[-p], V[-1-\alpha])\left[d_{X}\right]
$$

D'après 3.3.3-5, il suffit de vérifier que $\operatorname{Gr}^{V} \boldsymbol{D}\left(L^{\bullet}, F\right)$ est strict, que l'action de $t \partial_{t}-\alpha$ sur $H^{i} G r_{\alpha}^{V} \boldsymbol{D}\left(L^{\circ}\right)$ est nilpotente et que $H^{i}\left(F_{p} G r_{\alpha}^{V} \boldsymbol{D} L^{\circ}\right)=0$ pour $i \gg 0$, car ces conditions entraînent que $\boldsymbol{D}\left(L^{\circ}, F\right)$ est strict. On voit que toutes les conditions ne dépendent que du complexe de $G r^{V} \mathscr{D}$-modules gradués filtrés $G r^{V} \boldsymbol{D}\left(L^{\cdot}, F\right) \cong \mathbb{D}\left(G r^{V}\left(L^{\bullet}, F\right)\right)\left(\cong \mathbb{D}\left(G r^{V}(\tilde{M}, F)\right)\right)$. D'après le lemme ci-dessus, on obtient une filtration $W$ de $G r^{V} \tilde{M}$ (par des sous- $G r^{V} \mathscr{D}$-modules gradués) telle que $\operatorname{Gr}_{i}^{W} G r^{V}(\tilde{M}, F)$ est la somme directe de:

$$
G r_{i}^{W} G r_{-1}^{V}(\tilde{M}, F) \bigotimes(C[t], F), \quad G r_{i}^{W} G r_{0}^{V}(\tilde{M}, F) \bigotimes\left(C\left[\partial_{t}\right], F\right)
$$

et $\quad G r_{i}^{W} G r_{a}^{V}(\tilde{M}, F) \bigotimes\left(C\left[t, \partial_{t}\right] /\left(t \partial_{t}-\alpha\right) C\left[t, \partial_{t}\right], F\right) \quad(-1<\alpha<0)$,

donc l'assertion est réduite au cas où $\operatorname{Gr}^{V}(\tilde{M}, F)$ est isomorphe à un de tels modules. Puisque la résolution et le dual sont compatibles avec le produit $\bigotimes$, il suffit de vérifier les conditions pour $\operatorname{Gr}^{V}(\tilde{M}, F)=(\boldsymbol{C}[t], F)$, etc., car $\boldsymbol{D}\left(G r_{i}^{W} G r_{a}^{V}(\tilde{M}, F)\right)$ sont stricts par hypothèse de récurrence. Alors l'assertion est claire.

5.1.14. Proposition. Soit $M F_{h} W\left(\mathscr{D}_{X}, \boldsymbol{k}\right)$ la catégorie des objets de $M_{h}\left(\mathscr{D}_{X}, \boldsymbol{k}\right)$ munis d'une filtration croissante localement finie $W(c f .1 .3 .1)$, i.e. les objets de $M F_{h} W\left(\mathscr{D}_{X}, \mathbb{k}\right)$ sont des couples $((M, F, W),(K, W))$ munis d'un isomorphisme:

$$
\alpha: D R(M) \simeq C \otimes K \text { tel que } \alpha: D R\left(W_{i} M\right) \simeq \boldsymbol{C} \otimes W_{i} K .
$$

Soit $M H W(X, k)$ la sous-catégorie pleine de $M F_{h} W\left(\mathscr{D}_{X}, \mathbb{k}\right)$ des objets satisfaisant la condition: $G r_{i}^{W} \in M H(X, k, i)$ pour tout $i$. Alors $M H_{Z}(X, k, n)$, $M H(X, \boldsymbol{k}, n)$ et $M H W(X, \boldsymbol{k})$ sont des catégories abéliennes dont les morphismes sont toujours stricts pour $F$ ou pour $(F, W)$.

Preuve. L'assertion pour $M H(X, \mathbb{k}, n)$ est réduite à celle pour $M H_{Z}(X, \boldsymbol{k}, n)$ d'après 5.1.11. Considérons les assertions:

$a(i): \quad M H_{Z}(X, \boldsymbol{k}, n)$ est une catégorie abélienne dont les morphismes sont stricts, si $\operatorname{dim} Z \leqslant i$.

$b(i): \quad M H W(X, \boldsymbol{k})^{i}$ est une catégorie abélienne dont les morphismes sont stricts. 
où $M H W(X, \mathbb{R})^{i}$ est la sous-catégorie pleine de $M H W(X, \mathbb{k})$ des objets dont la dimension du support est $\leqslant i$. Alors l'assertion $a(0)$ est claire, et l'implication $a(i) \Rightarrow b(i)$ résulte de $1.2 .10,5.1 .11$ et du lemme 5.1 .15 ci-dessous, où on prend $M G_{h}\left(\mathscr{B}_{X}, \mathbb{R}\right)$ (cf. 5.1.1) pour $\mathcal{A}$. Prouvons $b(i) \Rightarrow a(i+1)$.

Soit $u:(M, F, K) \rightarrow\left(M^{\prime}, F, K^{\prime}\right)$ un morphisme de $M H_{Z}(X, \mathbb{R}, n)$, alors il faut montrer que $F$ est strict et que $\operatorname{Ker} u$, Coker $u \in M H_{Z}(X, \mathbb{R}, n)$. D'après $3.3 .3-5,3.2 .6,3.1 .6$ et 5.1.8, il suffit de vérifier que pour toute fonction $g$ (localement définie) telle que $\operatorname{dim} g^{-1}(0) \cap Z \leqslant i$,

$$
\psi_{g} u:\left(\psi_{g}(M, F, K), W\right) \rightarrow\left(\psi_{g}\left(M^{\prime}, F, K^{\prime}\right), W\right)
$$

est strict (de même pour $\phi_{g, 1}$ ) et

$$
\begin{aligned}
\operatorname{can}: & \operatorname{Ker} \psi_{g, 1} u \rightarrow \operatorname{Ker} \phi_{g, 1} u \\
\text { (resp. } \operatorname{Var}: \operatorname{Ker} \phi_{g, 1} u & \rightarrow \operatorname{Ker} \psi_{g, 1} u(-1) \text { ) }
\end{aligned}
$$

est surjectif (resp. injectif) (de même pour Coker), car on peut remplacer $(M, F, K)$ par la $Z^{\prime}$-composante (cf. 5.1.3.5) de $\left.(M, F, K)\right|_{U}$ dans 5.1.8, où $Z^{\prime}$ est une composante de $Z \cap U$. Mais la première résulte de $b(i)$ et la dernière de la décomposition de Lefschetz (par le passage à $G r^{W}$ ); d'où l'assertion.

5.1.15. Lemme. Soit $\mathcal{A}$ une catégorie abélienne, soient $\mathscr{A}_{j}$ des souscatégories additives pleines strictes de $\mathcal{A}(j \in \mathbb{Z})$, soit $\mathcal{A} W$ la catégorie des objets de $\mathcal{A}$ munis d'une filtration finie croissante $W$ telle que $G r_{j}^{W} \in A_{j}$ pour tout $j$. Supposons que chaque $A_{j}$ soit stable par $\operatorname{Ker}$ et $\operatorname{Coker}$ dans $\mathcal{A}$ (en particulier, $A_{j}$ est abélienne), et que $\operatorname{Hom}\left(\mathcal{A}_{j}, \mathscr{A}_{k}\right)=0$ pour $j>k$, alors $\mathcal{A} W$ est une catégorie abélienne dont les morphismes sont toujours stricts pour $W$.

Preuve. Soit $u:(E, W) \rightarrow\left(E^{\prime}, W\right)$ un morphisme de $\mathcal{A} W$. Si $u$ est strict, Ker et Coker commutent à $G r_{j}^{W}$, donc $\mathbb{K e r} u$, Coker $u \in \mathcal{A} W$ par hypothèse. Pour démontrer que $u$ est strict, on considère un diagramme dans $\mathcal{A} W$ :

$$
\begin{gathered}
0 \rightarrow W_{j-1}(E, W) \rightarrow W_{j}(E, W) \rightarrow G r_{j}^{W}(E, W) \rightarrow 0 \\
u \downarrow \\
u \downarrow \\
0 \rightarrow W_{j-1}\left(E^{\prime}, W\right) \rightarrow W_{j}\left(E^{\prime}, W\right) \rightarrow G r_{j}^{W}\left(E^{\prime}, W\right) \rightarrow 0,
\end{gathered}
$$

et on procède par récurrence d'après le lemme suivant:

5.1.16. Lemme. Soit $C$ une catégorie exacte, soit

$$
\begin{aligned}
0 \rightarrow & E^{\prime} \rightarrow E \rightarrow E^{\prime \prime} \rightarrow 0 \\
& \downarrow u^{\prime} \quad \downarrow u \quad \downarrow u^{\prime \prime} \\
0 \rightarrow & F^{\prime} \rightarrow F \rightarrow F^{\prime \prime} \rightarrow 0
\end{aligned}
$$


un morphisme entre des suites exactes courtes dans $\mathcal{C}$. Si $u^{\prime}$ et $u^{\prime \prime}$ sont stricts, et si Hom $\left(\operatorname{Ker} u^{\prime \prime}\right.$, Coker $\left.u^{\prime}\right)=0$, alors $u$ est aussi strict.

Preuve. Soit $\tilde{\mathcal{C}}$ comme dans 1.3 .2 , on a alors une suite exacte courte dans $\tilde{\mathcal{C}}$ :

$$
0 \rightarrow \operatorname{Ker} u^{\prime} \rightarrow \operatorname{Ker} u \rightarrow \operatorname{Ker} u^{\prime \prime} \rightarrow 0
$$

(de même pour Coker et Im) par le lemme du serpent; d'où $\operatorname{Ker} u$, Coker $u$, $\operatorname{Im} u \in \mathcal{C}$, i.e. $u$ est strict. Ce qui achève la preuve de 5.1.15 et de 5.1.14.

5.1.17. Lemme. Soient $(M, F, K) \in M F_{h}\left(\mathscr{D}_{X}, \boldsymbol{k}\right)$ et $g$ une fonction holomorphe sur $X$. Supposons que $(M, F)$ est quasi-unipotent et régulier le long de $g$ et que

$$
\text { can: } \left.\psi_{g, 1} \rightarrow \phi_{g, 1} \quad \text { (resp. Var: } \phi_{g, 1} \rightarrow \psi_{g, 1}(-1)\right)
$$

soit surjectif (resp. injectif), i.e. $(M, F), K$ et $(M, F, K)$ n'ont ni de sous-objets, ni d'objets quotients, à supports dans $g^{-1}(0)$, cf. 5.1.4. Soit $W$ la filtration de $\psi_{g, 1}$ et de $\phi_{g, 1}$ comme dans 5.1.6. Si $\left(\psi_{g, 1}(M, F, K), W\right) \in M H W(X, k)$, les conditions suivantes sont équivalentes:

(5.1.17.1) can: $\psi_{g, 1} \rightarrow \phi_{g, 1}$ est strict dans $M F_{h}\left(\mathscr{D}_{X}, \boldsymbol{k}\right)$,

(5.1.17.2) Var: $\phi_{g, 1} \rightarrow \psi_{g, 1}(-1)$ est strict dans $M F_{h}\left(\mathscr{D}_{X}, \boldsymbol{k}\right)$,

(5.1.17.3) can: $\left(\psi_{g, 1}, W\right) \rightarrow\left(\phi_{g, 1}, W\right)$ est strict dans $M F_{h} W\left(\mathscr{D}_{X}, \boldsymbol{k}\right)$,

(5.1.17.4) Var: $\left(\phi_{g, 1}, W\right) \rightarrow\left(\psi_{g, 1}, W\right)(-1)$ est strict dans $M F_{h} W\left(\mathscr{D}_{X}, \boldsymbol{k}\right)$,

(5.1.17.5) $\quad\left(\phi_{g, 1}(M, F, K), W\right) \in M H W(X, k)$,

ò̀ $\left(\psi_{g, 1}, W\right)(-1)=\left(\psi_{g, 1}(-1), W[2]\right)$. Si ces conditions sont vérifiées, on a les isomorphismes:

(5.1.17.6) can: $P_{N} G r_{i}^{W} \psi_{g, 1} \stackrel{\sim}{\rightarrow} P_{N} G r_{i}^{W} \phi_{g, 1}$ pour $i \geqslant n$,

où $P_{N} G r_{n-1+i}^{W} \psi_{g, 1}^{\prime}=\operatorname{Ker} N^{i+1} \subset G r_{n-1+i}^{W} \psi_{g, 1}$ (de même pour $\phi_{g, 1}$ en remplaçant $n-1 \operatorname{par} n)$.

Preuve. D'abord notons que 5.1.12 entraîne que les morphismes en (5.1.17.34) sont bien-définis. D'après 5.1 .14 , les implications: $(5.1 .17 .5) \Rightarrow(5.1 .17 .3) \Rightarrow$ (5.1.17.1) et $(5.1 .17 .5) \Rightarrow(5.1 .17 .4) \Rightarrow(5.1 .17 .2)$ sont claires, donc il suffit de vérifier: (5.1.17.1) (ou (5.1.17.2)) $\Rightarrow(5.1 .17 .5)$. Si (5.1.17.1) ou (5.1.17.2) est vérifiée, on a l'isomorphisme:

$$
\text { Coim } N \simeq\left(\phi_{g, 1}, W\right) \text { dans } M F_{h} W\left(\mathscr{D}_{X}, k\right) \text {, }
$$


par la factorisation $N=$ Varocan. En fait (5.1.17.7) résulte de 5.1.12, si on oublie la filtration $F$, et l'assertion pour $F$ résulte du fait que $N: \psi_{g, 1} \rightarrow \psi_{g, 1}(-1)$ est strict pour $F$ d'après 5.1.14. Alors (5.1.17.5) résulte de (5.1.17.7) et de 5.1.14. Dans ce cas, on a $G r^{W} \phi_{g, 1}=$ Coim $G r^{W} N$; d'où (5.1.17.6) par la décomposition de Lefschetz.

5.1.18. Remarque. Soit $(M, F, K) \in M H(X, \mathbb{k}, n)$, soit $f$ un morphisme d'un disque $D$ dans $X$; alors on peut vérifier, par récurrence sur dim supp M, que $\mathscr{H}^{i} f^{!} M$ sont des $\mathscr{D}$-Modules holonomes réguliers, quasi-unipotents, i.e. $M$ est régulier, et quasi-unipotent (au sens de Kashiwara.)

\subsection{Polarisation}

5.2.1. Soit $X$ une variété complexe lisse, soit $p: X \rightarrow D$ un morphisme lisse dans un disque $D$, posons

$$
\begin{aligned}
& i: X_{0}=p^{-1}(0) \rightarrow X, \quad j: X^{*}=X \backslash X_{0} \rightarrow X, \\
& \tilde{j}: \tilde{X}^{*}=X \times_{D} \tilde{D}^{*} \rightarrow X,
\end{aligned}
$$

où $\tilde{D}^{*} \rightarrow D^{*}=D \backslash\{0\}$ est un revêtement universel. Soit $K$ un objet de $D_{c}^{b}\left(\mathbb{R}_{X}\right)$ représenté par un complexe dont les composantes sont flasques (i.e. injectives sur $\mathbb{R}_{X}$ ). On définit

$$
\begin{aligned}
& \psi K=i^{*} \tilde{j}_{*} \tilde{j}^{*} K, \quad{ }^{p} \psi K=\psi K[-1], \\
& { }^{p} \phi_{1} K=C\left(\mathrm{sp}: i^{*} K[-1] \rightarrow{ }^{p} \psi_{1} K\right) \in D_{c}^{b}\left(\mathbb{R}_{X_{0}}\right) .
\end{aligned}
$$

Ici, ${ }^{p} \psi_{1} K=\bigcup_{i} \operatorname{Ker}(T-\mathrm{id})^{i} \subset^{p} \psi K K$ comme dans 3.4 .14 , et sp est un élément de $\operatorname{Ext}^{1}\left(i * K,{ }^{p} \psi_{1} K\right)$ représenté par le morphisme naturel: $i * K \rightarrow{ }^{p} \psi_{1} K[1] \subset$ $i * \tilde{j}_{*} \tilde{j}^{*} K$. On a alors un triangle dans $D_{c}^{b}\left(\mathbb{k}_{X_{0}}\right)$

$$
\rightarrow{ }^{p} \psi_{1} K \stackrel{\text { can }}{\longrightarrow}{ }^{p} \phi_{1} K \rightarrow i^{*} K \stackrel{+1}{\longrightarrow} \text {. }
$$

Puisque $0 \rightarrow i^{*} j_{*} j^{*} K \rightarrow \psi_{1} K \stackrel{N}{\rightarrow} \psi_{1} K(-1) \rightarrow 0$ est exacte dans la catégorie de complexes, on a un quasi-isomorphisme:

$$
i^{!} K \stackrel{\mathrm{sp}}{\rightarrow}\left[i^{*} K \stackrel{-N}{\rightarrow} \psi_{1} K \stackrel{-}{\longrightarrow} \psi_{1} K(-1)\right]
$$

où le terme à droite est le complexe simple associé au complexe double tel que $i^{*} K$ est de degré 0 . Désormais $i^{1} K$ sera représenté par le but du morphisme (5.2.1.3), alors le morphisme naturel: $i^{!} K \rightarrow i^{*} K$ s'identifie à la projection (id, 0,0$)$. On définit cosp $\in \operatorname{Ext}^{1}\left({ }^{p} \psi_{1} K(-1), i^{!} K\right)$ par le morphisme naturel:

$$
{ }^{p} \psi_{1} K(-1)[-1]=\psi_{1} K(-1)[-2] \stackrel{(0,0, \mathrm{id})}{\longrightarrow}\left[i^{*} K \rightarrow \psi_{1} K \rightarrow \psi_{1} K(-1)\right],
$$


on a alors un quasi-isomorphisme naturel:

$$
C\left(\operatorname{cosp}:{ }^{p} \psi_{1} K(-1)[-1] \rightarrow i^{!} K\right) \rightarrow{ }^{p} \phi_{1} K,
$$

donc on obtient le triangle dans $D_{c}^{b}\left(\boldsymbol{k}_{X_{0}}\right)$ :

$$
\rightarrow i^{!} K \rightarrow{ }^{p} \phi_{1} K \stackrel{\operatorname{Var}^{p}}{\longrightarrow} \psi_{1} K(-1) \stackrel{+1}{\longrightarrow} \text {. }
$$

Notons que le morphisme Var dans (5.2.1.4) coïncide (dans $D_{c}^{b}\left(\boldsymbol{k}_{X_{0}}\right)$ ) avec le morphisme:

$$
(0, N):{ }^{p} \phi_{1} K=\left[i * i^{*} \operatorname{sp}_{\rightarrow} \psi_{1} K\right] \rightarrow \psi_{1} K[-1]={ }^{p} \psi_{1} K,
$$

défini comme dans 3.4, et que la composition $i^{!} K \rightarrow^{p} \phi_{1} K \rightarrow i^{*} K$ des morphismes dans (5.2.1.2) et (5.2.1.4) est le morphisme naturel: $i^{!} K \rightarrow i^{*} K$.

5.2.2. Remarque. Avec les notations ci-dessus, les triangles (5.2.1.2), (5.2.1.4) (et les remarques ci-dessus) se déduisent un diagramme de l'octaèdre (cf. [1, (1.1.6)]):

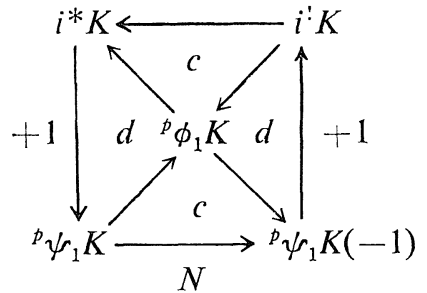

(calotte supérieure)

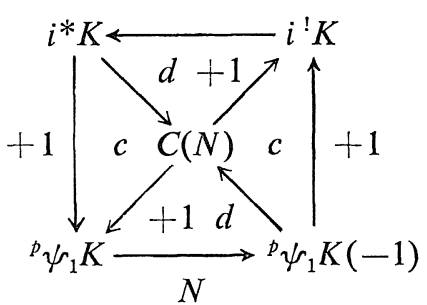

(calotte inférieure)

où $d$ signifie "distingué" et $c$ "commutatif". Alors les (deux) suites longues associées au pourtour de ces deux carrés:

$$
\rightarrow^{p} \mathcal{H}^{i}\left({ }^{p} \psi_{1} K\right) \stackrel{N}{\rightarrow}{ }^{p} \mathcal{H}^{i}\left({ }^{p} \psi_{1} K(-1)\right) \rightarrow{ }^{p} \mathcal{H}^{i+1}\left(i^{1} K\right) \rightarrow{ }^{p} \mathscr{H}^{i+1}\left(i^{*} K\right) \rightarrow
$$

sont dites la suite longue de Clemens-Schmid [5]. (Notons que la calotte inférieure (resp. supérieure) est utilisée par Clemens-Schmid (resp. Zucker) dans le cas $K=f_{*} C_{Y}$ pour $f: Y \rightarrow D$ un morphisme propre de Kähler, et que les foncteures ${ }^{p} \psi_{1}, i^{*}$, etc. commutent au foncteur image directe propre.) D'adrès Zucker (5.2.2.2) sont exactes sii:

$$
\begin{aligned}
{ }^{p} \mathcal{H}{ }^{i}\left({ }^{p} \phi_{1} K\right)= & \operatorname{Im}\left(\operatorname{can}:{ }^{p} \mathcal{H}^{i}\left({ }^{p} \psi_{1} K\right) \rightarrow{ }^{p} \mathcal{H}^{i}\left({ }^{p} \phi_{1} K\right)\right) \\
& \oplus \operatorname{Ker}\left(\operatorname{Var}:{ }^{p} \mathcal{H}^{i}\left({ }^{p} \phi_{1} K\right) \rightarrow{ }^{p} \mathscr{H}^{i}\left({ }^{p} \psi_{1} K(-1)\right)\right) \text { pour tout } i,
\end{aligned}
$$

(comparer à la condition (5.1.4.2).) Cette condition est vérifieé si $K$ est une 
somme directe de faisceaux pervers $K^{\prime}$ (décalés) tels qu'il n'y a pas de sousobjets (ni d'objets quotients) de $K^{\prime}$ à supports dans $X_{0}$ (i.e. can est surjectif et Var est injectif), ou que supp $K^{\prime} \subset X_{0}$ (car ${ }^{p} \psi_{1}$ et ${ }^{p} \phi_{1}$ commutent à $\left.{ }^{p} \mathscr{H}^{i}\right)$. Dans ce cas, (5.2.2.2) est une somme directe de suites exactes:

ou

$$
0 \rightarrow \mathbb{K e r} N \rightarrow{ }^{p} \psi_{1} K^{\prime} \stackrel{N}{\rightarrow}{ }^{p} \psi_{1} K^{\prime}(-1) \rightarrow \operatorname{Coker} N \rightarrow 0
$$

Par exemple, on a

$$
f_{*} \mathbb{C}_{Y}=\oplus_{k}\left(j_{*} j^{*} \mathcal{H}^{k} f_{*} \mathbb{C}_{Y}\right)[-k] \oplus \oplus_{k} i_{*} \operatorname{Ker}\left(H^{k}\left(Y_{0}\right) \rightarrow H^{k}\left(Y_{t}\right)\right)[-k]
$$

pour $f: Y \rightarrow D$ comme ci-dessus, où $f$ est lisse sur $D^{*}$ et $t \in D^{*}$; alors ${ }^{p} \psi_{1} K^{\prime}=$ $\mathbb{H}^{k}\left(Y_{t}\right)$ ou $i^{*} K^{\prime}=\operatorname{Ker}\left(H^{k}\left(Y_{0}\right) \rightarrow H^{k}\left(Y_{t}\right)\right)$ pour $k \in \mathbb{Z}$.

5.2.3. Avec les notations de 5.2.1, soient $K, L \in D_{c}^{b}\left(\mathbb{R}_{X}\right)$ et $S: K \otimes \mathbb{L} \rightarrow a_{X}^{!} \mathbb{R}(r)$ un accouplement, où $a_{X}: X \rightarrow p t$. Ici on suppose que $K, L$ et $a_{X}^{!} \mathbb{R}(r)\left(\cong \mathbb{K}_{X}\left(r+d_{X}\right)\right.$ $\left.\left[2 d_{X}\right]\right)$ sont représentés par des complexes dont les composantes sont flasques. On dit que $S$ est non-dégénéré si le morphisme correspondant: $K \rightarrow \mathbb{D}(L)$ est un quasi-isomorphisme. On définit

$$
\psi S: \psi K \otimes \psi L \rightarrow \psi a_{X}^{!} \mathbb{R}(r)\left(\cong a_{X_{0}}^{!} \mathbb{k}(r+1)[2]\right)
$$

par $\quad i^{*} \tilde{j}_{*} \tilde{j}^{*} K \otimes i^{*} \tilde{j}_{*} \tilde{j}^{*} L \rightarrow i^{*} \tilde{j}_{*}\left(\tilde{j}^{*} K \otimes \tilde{j}^{*} L\right) \rightarrow i^{*} \tilde{j}_{*} \tilde{j}^{*} a_{X}^{!} \mathbb{R}(r)$, et

$$
{ }^{p} \psi S:{ }^{p} \psi K \otimes \otimes^{p} \psi L \rightarrow \psi a_{X}^{!} \mathbb{k}(r)[-2] \quad\left(\cong a_{X_{0}}^{!} k(r+1)\right)
$$

de sorte que

$$
\begin{aligned}
\psi K \otimes \otimes \psi & =\left(\mathbb{k}[1] \otimes^{p} \psi K\right) \otimes\left(\mathbb{R}[1] \otimes^{p} \psi L\right) \\
& \cong(\mathbb{R}[1] \otimes \mathbb{E}[1]) \otimes\left({ }^{p} \psi K \otimes^{p} \psi L\right) \stackrel{{ }^{p} \psi S}{\longrightarrow} \psi a_{X}^{!} \mathbb{R}(r)
\end{aligned}
$$

coïncide avec $\psi S$, où le deuxième isomorphisme est définie par

$$
x \otimes y \otimes z \otimes w \rightarrow(-1)^{\operatorname{deg} y \cdot \operatorname{deg} z} x \otimes z \otimes y \otimes w, \quad \text { cf. [9]. }
$$

Alors $\psi S$ induit $\psi_{1} S: \psi_{1} K \otimes \psi_{1} L \rightarrow \psi_{1} a_{X}^{!} \mathbb{k}(r)\left(\cong a_{X_{0}}^{!} \mathbb{R}(r+1)\right.$ [2]) (de même pour $\left.{ }^{p} \psi_{1} S\right)$. On définit

$$
i^{!} S: i^{*} K \otimes i^{!} L \rightarrow i^{1} a_{X}^{1} \mathbb{k}(r)\left(\cong a_{X_{0}}^{1} \mathbb{E}(r)\right)
$$

par

$i^{*} K \otimes\left[i^{*} L \rightarrow \psi_{1} L \rightarrow \psi_{1} L(-1)\right] \rightarrow\left[i^{*}(K \otimes L) \rightarrow \psi_{1}(K \otimes L) \rightarrow \psi_{1}(K \otimes L)(-1)\right]$

$\stackrel{\left(i^{*} S, \psi S, \psi S\right)}{\longrightarrow}\left[i^{*} a_{X}^{!} \mathbb{R}(r) \rightarrow \psi_{1} a_{X}^{!} \mathbb{k}(r) \rightarrow \psi_{1} a_{X}^{!} \mathbb{k}(r-1)\left(\approx i^{!} a_{X}^{!} \mathbb{k}(r)\right)\right.$

où on utilise l'isomorphisme (5.2.1.3). Notons que le morphisme 


$$
(0,0, i d): \psi_{1} a_{X}^{!} k(r-1)[-2] \rightarrow\left[i * a_{X}^{!} k(r) \rightarrow \psi_{1} a_{X}^{!} k(r) \rightarrow \psi_{1} a_{X}^{!} k(r-1)\right]
$$

s'identifie à l'identité par les isomorphismes canoniques:

$$
\psi_{1} a_{X}^{!} k(r-1)[-2] \cong a_{X_{0}}^{!} k(r) \text { et } i^{!} a_{X}^{1} k(r) \cong a_{X_{0}}^{1} k(r) .
$$

Alors on vérifie que les morphismes $\psi_{1} S(\mathrm{sp} \otimes i d)$ et $i^{1} S(i d \otimes \operatorname{cosp})$ de $i^{*} K \otimes$ $\psi_{1} L(-1)[-2]$ dans le but du morphisme (5.2.3.1) coïncident, où le décalage [-2] n'intervient pas au signe. D'après le lemme suivant on obtient l'accouplement

$$
{ }^{p} \phi_{1} S:{ }^{p} \phi_{1} K \otimes{ }^{p} \phi_{1} L \rightarrow a_{X_{0}}^{!} k(r)
$$

compatible avec ${ }^{p} \psi_{1} S$ et $i^{\prime} S$, i.e. l'accouplement des triangles (5.2.1.2) pour $K$ et (5.2.1.4) pour $L$.

5.2.4. Lemme. Soient $u: B[-1] \rightarrow A$ (resp. $\left.u^{\prime}: A^{\prime}[-1] \rightarrow B^{\prime}\right)$ un morphisme de complexes, et $C$ (resp. $\left.C^{\prime}\right)$ le cône de $u$ (resp. $\left.u^{\prime}\right)$. Soient $S_{A}: A \otimes A^{\prime} \rightarrow D$ et $S_{B}: B \otimes B^{\prime} \rightarrow D$ des morphismes de complexes. On définit $S_{A}^{\prime}$ par la composition:

$$
\begin{aligned}
A[1] \otimes A^{\prime}[-1] & =\boldsymbol{Z}[1] \otimes A \otimes Z[-1] \otimes A^{\prime} \cong \mathbb{Z}[1] \otimes Z[-1] \otimes A \otimes A^{\prime} \\
& =A \otimes A^{\prime} \stackrel{S_{A}}{\longrightarrow} D .
\end{aligned}
$$

Si $S_{A}^{\prime}(u \otimes i d)=S_{B}\left(i d \otimes u^{\prime}\right): B \otimes A^{\prime}[-1] \rightarrow D, S_{A} \oplus S_{B}$ définit un accouplement $S_{C}$ :

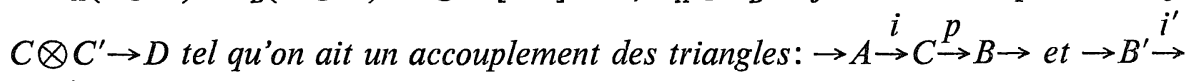
$C^{\prime} \stackrel{p^{\prime}}{\rightarrow} A^{\prime} \rightarrow$, i.e.

$$
S_{C}(i \otimes i d)=S_{A}\left(i d \otimes p^{\prime}\right) ; A \otimes C^{\prime} \rightarrow D, \quad S_{C}\left(i d \otimes i^{\prime}\right)=S_{B}(p \otimes i d): C \otimes B^{\prime} \rightarrow D .
$$

Preuve. Soient $(a, b) \in C^{i}=A^{i} \oplus B^{i},\left(a^{\prime}, b^{\prime}\right) \in C^{\prime j}=A^{\prime j} \oplus B^{\prime j}$, alors $S_{C}((a, b)$, $\left.\left(a^{\prime}, b^{\prime}\right)\right)=S_{A}\left(a, a^{\prime}\right)+S_{B}\left(b, b^{\prime}\right)$ par définition. On vérifie que $S_{C}$ est un morphisme de complexes sii $S_{A}\left(u b, a^{\prime}\right)+(-1)^{i} S_{B}\left(b, u^{\prime} a^{\prime}\right)=0$; mais $S_{A}^{\prime}\left(u b, a^{\prime}\right)=(-1)^{i+1} S_{A}$ $\left(u b, a^{\prime}\right)$ car $u b \in A^{i+1}$; d'où l'assertion.

5.2.5. Lemme. Avec les notations 5.2.3, soient $T$ la monodromie, $T=T_{s} T_{u}$ la décomposition de Jordan et $N=\left(\log T_{u}\right) / 2 \pi i$ sur $\psi K\left(\cong \bigoplus_{P}(\psi K)\right)$ et $\phi_{1} K$ (de même pour L), cf. 3.4.14. On a alors

$$
{ }^{p} \psi S \circ(N \otimes i d)+{ }^{p} \psi S \circ(i d \otimes N)=0
$$

(de même pour $\left.{ }^{p} \phi_{1}\right)$ dans $D_{c}^{b}\left(\boldsymbol{k}_{X_{0}}\right)$.

Preuve. Cela résulte du diagramme commutatif dans $D_{c}^{b}\left(\boldsymbol{k}_{X_{0}}\right)$ suivant: 


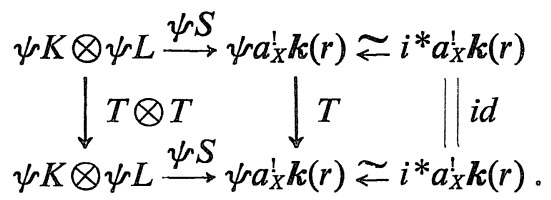

5.2.6. Corollaire. Avec les notations ci-dessus, supposons que $K$ et $L$ soient des faisceaux pervers. Soit $W$ la filtration de ${ }^{p} \psi K,{ }^{p} \phi_{1} K$, etc. associée à $N$ décalée par 0 , alors la composition:

$$
W_{i}^{p} \psi K \otimes W_{j}^{p} \psi L \rightarrow{ }^{p} \psi K \otimes \otimes^{p} \psi L \rightarrow a_{X_{0}}^{!} k(r+1)
$$

est nulle dans $D_{c}^{b}\left(\boldsymbol{k}_{X_{0}}\right)$ pour $i+j<0$ (de même pour $\left.\phi_{1}\right)$, donc on a des accouplements:

$$
\begin{aligned}
& G r^{W p} \psi S: G r_{i}^{W p} \psi K \otimes G r_{-i}^{W} \psi L \rightarrow a_{X_{0}}^{!} k(r+1) \\
& G r^{W p} \phi_{1} S: G r_{i}^{W p} \phi_{1} K \otimes G r_{-i}^{W} \phi_{1} L \rightarrow a_{X_{0}}^{!} k(r) .
\end{aligned}
$$

Preuve. L'accouplement ${ }^{p} \psi S:{ }^{p} \psi K \otimes^{p} \psi L \rightarrow a_{X_{0}}^{!} k(r+1)$ correspond à un morphisme dans Perv $\left(\boldsymbol{k}_{X_{0}}\right)$ :

$$
{ }^{p} \psi K \rightarrow D^{p} \psi L(r+1)
$$

Si on définit l'action de $N$ sur $\mathbb{D}^{p} \psi L$ par $-N^{*}$, ce morphisme est $N$-linéaire d'après 5.2.5. Puisque la filtration associée (décalée par 0 ) est auto-duale, l'assertion résulte de 1.3.9-10.

5.2.7. Lemme. Soient $K, L$ et $S$ comme dans 5.2.3. Soit ${ }^{p} \mathcal{H}^{j}(j \in \mathbb{Z})$ le foncteur cohomologique pervers [1], alors $S$ induit canoniquement:

$$
{ }^{p} \mathcal{H} S:{ }^{p} \mathcal{H}^{-j} K \otimes^{p} \mathcal{H}^{j} L \rightarrow a_{X}^{!} k(r) .
$$

On a de plus ${ }^{p} \mathcal{H}^{p} \psi S={ }^{p} \psi^{p} \mathcal{H} S$ par les isomorphismes canoniques:

$$
\left.{ }^{p} \mathcal{H}^{j p} \psi K \simeq{ }^{p} \psi^{p} \mathcal{H}^{j} K \text {, etc. (de même pour } \phi_{1}\right)
$$

Preuve. Cela résulte du fait que ${ }^{p} \psi,{ }^{p} \phi_{1}$ sont des foncteurs $t$-exacts et que ${ }^{p} \mathcal{H} S$ est uniquement déterminé par le diagramme commutatif:

$$
\begin{aligned}
& { }^{p} \tau_{\leqq-j} K \otimes{ }^{p} \tau_{\leqq j} L \rightarrow{ }^{p} \mathscr{H}^{-j} K \otimes^{p} \mathscr{H}^{j} L
\end{aligned}
$$

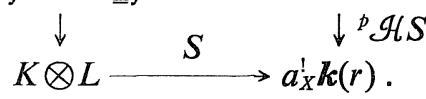

5.2.8. Lemme. Avec les notations de 5.2.3, supposons que supp $K$, supp $L \subset X_{0}$, alors $S: K \otimes L \rightarrow a_{X}^{!} k(r)$ se factorise canoniquement par

$$
S^{\prime}: K \otimes L \rightarrow \underline{\Gamma}_{X_{0}} a_{X}^{!} \boldsymbol{k}(r) \quad\left(=i_{*} i^{!} a_{X}^{!} k(r)\right)
$$


qui s'identifie à l'image directe $\left(\right.$ par $\left.i_{*}\right)$ de

$$
i^{!} S: i^{*} K \otimes i^{!} L \rightarrow i^{!} a_{X}^{!} \boldsymbol{k}(r)
$$

par l'isomorphisme canonique: $i_{*} i^{1} L \underset{\rightarrow}{\longrightarrow} L$, donc ${ }^{p} \phi_{1} S$ s'identifie canoniquement à $S, c f .5 .2 .3-4$.

Preuve. D'abord notons que $a_{X}^{!} \boldsymbol{k}(r)$ est représenté par un complexe de faisceaux flasques; d'où la première assertion. Les autres résultent de la définition de $i^{\prime} S$, de l'isomorphisme (5.2.1.3) pour $L$ et pour $a_{X}^{!} k(r)$, et de 5.2.4.

5.2.9. Lemme. Avec les notations de 5.2.1 et 5.2.3, soit $f: X \rightarrow Y$ un morphisme propre de variétés complexes lisses, qui factorise $p: X \rightarrow D$, i.e. $p=q f$ avec $q: Y \rightarrow D$ lisse. Soit $\operatorname{Tr}: a_{X}^{!} \boldsymbol{k} \rightarrow a_{Y}^{!} \boldsymbol{k}$ le morphisme de trace. Pour simplifier la notation, $f$ (resp. $i$ ) notera encore $f: X_{0} \rightarrow Y_{0}$ (resp. $Y_{0} \hookrightarrow Y$ ). On a alors les isomorphismes canoniques:

$$
i^{*} f_{*} K \cong f_{*} i^{*} K,{ }^{p} \psi_{(1)} f_{*} K \cong f_{*}^{p} \psi_{(1)} K, \quad i^{l} f_{*} K \cong f_{*} i^{!} K, \quad{ }^{p} \phi_{1} f_{*} K \cong f_{*}^{p} \phi_{1} K
$$

(de même pour L et $a_{X}^{!} \boldsymbol{k}$ ) et les images directes des accouplements:

$$
\begin{aligned}
& f_{*}^{p} \psi_{(1)} S: f_{*}^{p} \psi_{(1)} K \otimes f_{*}^{p} \psi_{(1)} L \rightarrow i^{!} a_{Y}^{!} \boldsymbol{k}(r+1), \\
& \left.f_{*} i^{!} S: f_{*} i^{*} K \otimes f_{*} i^{!} L \rightarrow i^{!} a_{Y}^{!} \boldsymbol{k}(r) \quad \text { (de même pour } f_{*}^{p} \phi_{1} S\right)
\end{aligned}
$$

qui s'identifient respectivement $\grave{a}^{p} \psi_{(1)} f_{*} S, i^{!} f_{*} S\left(\right.$ et $\left.{ }^{p} \phi_{1} f_{*} S\right)$, où $f_{*} S$ est définie par la composition:

$$
f_{*} K \otimes f_{*} L \rightarrow f_{*}(K \otimes L) \stackrel{S}{\rightarrow} f_{*} a_{X}^{!} \boldsymbol{k}(r) \stackrel{\operatorname{Tr}}{\rightarrow} a_{Y}^{!} k(r) .
$$

Preuve. Puisque $f$ est propre, on a la première assertion, où le foncteur $i^{!}$est representé toujours par $\left[i^{*} \rightarrow \psi_{1} \rightarrow \psi_{1}(-1)\right]$, cf. (5.2.1.3). (Ici tous les complexes $K, L, a_{X}^{!} \boldsymbol{k}$ et $a_{Y}^{!} \boldsymbol{k}$ sont représentés par des complexes de faisceaux flzasques.) Puisque

$$
\psi_{(1)} \operatorname{Tr}: \psi_{(1)} f_{*} a_{X}^{!} k(-1)[-2] \rightarrow \psi_{(1)} a_{Y}^{!} k(-1)[-2] \text { et } i^{!} \operatorname{Tr}: i^{!} f_{*} a_{X}^{!} k \rightarrow i^{!} a_{Y}^{!} k
$$

représentent le morphisme trace: $f_{*} a_{X_{0}} k \rightarrow a_{Y_{0}}^{\prime} k$ par les isomorphismes dans la première assertion et par l'identification (5.2.3.1), on obtient les images directes de ${ }^{p} \psi_{(1)} S, i^{!} S$ et de ${ }^{p} \phi_{1} S$; alors la dernière assertion est claire par la définition de ces images directes.

5.2.10. Soient $(M, F, K) \in M F_{l}\left(\mathscr{D}_{X}, \boldsymbol{k}\right)_{(0)}$, cf. 5.1.6, et $(M, F, K)=\oplus\left(M_{Z}, F, K_{Z}\right)$ la décomposition par supports stricts, cf. (5.1.3.5). Soit $S: K \otimes K \rightarrow a_{X}^{\prime} k(r)$ un accouplement, alors $S$ est compatible avec cette décomposition, i.e. $S=\oplus S_{Z}$ 
dans $D_{c}^{b}\left(\mathbb{k}_{X}\right)$, où $S_{Z}: K_{Z} \otimes K_{Z} \rightarrow a_{X}^{1} \mathbb{k}(r)$ (dit la $Z$-composante de $S$ ), car $\operatorname{Hom}\left(K_{Z}, K_{Z^{\prime}}\right)=0$ pour $Z \neq Z^{\prime}$. On dit que $S$ est compatible avec la filtration $F$, s'il existe un (unique) morphisme:

$$
(M, F, K) \rightarrow \mathbb{D}(M, F, K)(r)
$$

dont la deuxième composante: $K \rightarrow \mathbb{D K}(r)$ correspond à $S$ par l'isomorphisme canonique:

$$
\operatorname{Hom}\left(K \otimes K, a_{X}^{!} \not{k}(r)\right)=\operatorname{Hom}\left(K, \operatorname{Hom}\left(K, a_{X}^{!} \not k(r)\right)\right.
$$

Soit $(M, F, K) \in M H(X, \mathbb{R}, n)$. On dit que $(M, F, K)$ est polarisable, s'il existe un accouplement $S: K \otimes K \rightarrow a_{X}^{!} \mathbb{k}(-n)$ compatible avec la filtration $F$, dit une polarisation de $(M, F, K)$, qui satisfait les conditions suivantes (définies par récurrence sur $\operatorname{dim} \operatorname{supp} M)$ :

Pour tous $U, g$ et $Z$ dans 5.1 .8 , soit $\left(M_{Z}, F, K_{Z}\right)$ la $Z$-composante de $\left.(M, F, K)\right|_{U}$, cf. (5.1.3.5), on a alors:

(5.2.10.1) Si $Z=\{x\}$ (donc $\left(M_{Z}, F, K_{Z}\right)=i_{x^{*}}\left(H_{C}, F, H_{k}\right)$ d'après (5.1.6.1)), il existe une polarisation $S^{\prime}$ de $\left(H_{C}, F, H_{k}\right)$ au sens de [6] (i.e. $\left.S^{\prime}(x, C \bar{x}) \geqq 0\right)$ telle que $S=i_{x^{*}} S^{\prime}$, cf. 5.2.9.

(5.2.10.2) Si $\operatorname{dim} Z>0$ (donc $g^{-1}(0) \supset Z, \quad$ cf. 5.1.8), $\quad G r^{W p} \psi_{g} S_{Z} \circ\left(i d \otimes N^{i}\right)$ : $P_{N} G r_{n-1+i}^{W} \psi_{g} K_{Z} \otimes P_{N} G r_{n-1+i}^{W} \psi_{g} K_{Z} \rightarrow a_{U}^{!} k(1-n-i)$ (cf. 5.2.6) est une polarisation de la partie primitive $P_{N} G r_{n-1+i}^{W} \psi_{g}\left(M_{Z}, F, K_{Z}\right)$, cf. (5.1.17.6), pour $i \geqq 0$, où $S_{Z}: K_{Z} \otimes K_{Z} \rightarrow a_{U}^{!} \mathbb{K}(-n)$ s'identifie à $i_{g^{*}} S_{Z}$ : $i_{g^{*}} K_{Z} \otimes i_{g^{*}} K_{Z} \rightarrow a_{U \times C}^{!} k(-n)$ pour la définition de ${ }^{p} \psi_{g} S_{Z}$, cf. 5.2.3.

D'après 5.2.4, on a ${ }^{p} \phi_{g, 1}(\operatorname{can} \otimes i d)={ }^{p} \psi_{g, 1}(i d \otimes \operatorname{Var})$, donc (5.2.10.2) et (5.1.17.6) entraînent:

$$
\begin{aligned}
& G r^{W p} \phi_{g, 1} S_{Z} \circ\left(i d \otimes N^{i}\right): P_{N} G r_{n+i}^{W} \phi_{g, 1} K_{Z} \otimes P_{N} G r_{n+i}^{W} \phi_{g, 1} K_{Z} \rightarrow a_{U}^{!} \mathbb{k}(-n \\
& -i) \text { est une polarisation de } P_{N} G r_{n+i}^{W} \phi_{g, 1}\left(M_{Z}, F, K_{Z}\right) \text { pour } i \geqq 0 .
\end{aligned}
$$

Dans le cas $g^{-1}(0) \supset Z,{ }^{p} \phi_{g .1} S_{Z}$ s'identifie canoniquement à $S_{Z}$ d'après 3.2.6, 5.2.8, (car $S: K_{Z} \otimes K_{Z} \rightarrow a_{U}^{!} \mathbb{R}(-n)$ se factorise par $a_{Z}^{\vdots} \mathbb{k}(-n) \rightarrow a_{U}^{!} \mathbb{k}(-n)$ canoniquement) donc (5.2.10.3) reste valable (car $N=0$ ).

5.2.11. Lemme. Ayec les notations de 5.1.9, S est une polarisation de $(M, F, K) \in M H_{Z}(X, \mathbb{k}, n)$ sii $i_{*} S$ est une polarisation de $i_{*}(M, F, K)$.

(C'est clair.)

5.2.12. Lemme. Avec les notations et les hypothèses de 5.1.10, soit $S$ une polarisation de $(M, F, K) \in M H_{Z}(X, k, n)$. Alors $S$ est $(-1)^{n}$-symé- 
trique (i.e. $S \circ \iota=(-1)^{n} S$, où $\iota: K \otimes K \rightarrow K \otimes K$ est défini par $\iota(x \otimes y)=$

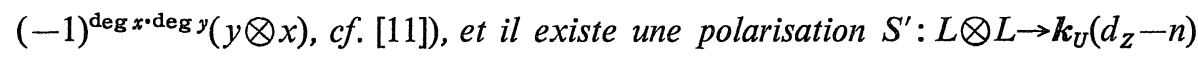
de la variation de structures de Hodge, telle que $\left.S\right|_{X \backslash(z \backslash U)}=i_{*} S^{\prime \prime}$, où

$$
S^{\prime \prime}: L\left[d_{z}\right] \otimes L\left[d_{z}\right] \rightarrow k_{U}\left(d_{z}-n\right)\left[2 d_{z}\right] \quad\left(\simeq a_{U}^{!} k(-n)\right)
$$

est défini par $(-1)^{d_{Z}\left(d_{Z}-1\right) / 2} S^{\prime}$ sur $L \otimes L=\left(L\left[d_{Z}\right] \otimes L\left[d_{Z}\right]\right)^{-2 d_{Z}}$.

Preuve. On peut supposer que $U=X$ (donc $\left.K=L\left[d_{X}\right]\right)$, alors la première assertion résulte de la dernière, car $S^{\prime}$ est $(-1)^{n-d_{X}}$-symétrique. Soient $x_{1}$ et $i$ : $X_{0} \rightarrow X$ comme dans la preuve de 5.1.10, alors

et

$$
\psi_{x_{1}} S: i^{*} L \otimes i^{*} L\left(=\left(\psi_{x_{1}} K \otimes \psi_{x_{1}} K\right)^{-2 d} x\right) \rightarrow k_{X_{0}}\left(d_{X}-n\right)
$$

$$
{ }^{p} \psi_{x_{1}} S: i^{*} L \otimes i^{*} L\left(=\left({ }^{p} \psi_{x_{1}} K \otimes{ }^{p} \psi_{x_{1}} K\right)^{2-2 d_{x}}\right) \rightarrow \boldsymbol{k}_{X_{0}}\left(d_{X_{0}}+1-n\right)
$$

coïncident au signe $(-1)^{d} x^{-1}$ près; d'où l'assertion par récurrence $\left(\operatorname{car} i * S=\psi_{x_{1}} S\right)$.

5.2.13. Corollaire. Soit $M H_{Z}(X, \boldsymbol{k}, n)^{p}$ la sous-catégorie pleine de $M H_{Z}$ $\times(X, \boldsymbol{k}, n)$ des objets polarisables, alors $M H_{Z}(X, \boldsymbol{k}, n)^{p}$ est semi-simple.

(Cela résulte de 3.2.2, 5.1.7 et de 5.1.10)

5.2.14 Proposition. Soient $(M, F, K) \in M F\left(\mathscr{D}_{X}, \mathbb{k}\right), S: K \otimes K \rightarrow a_{X}^{1} \mathbb{k}(-n)$ un accouplement, et $g$ une fonction holomorphe sur $X$. Supposons que $(M, F)$ soit quasi-unipotent et régulier le long de g, que

$$
\begin{aligned}
& \operatorname{can}\left(W_{i} \psi_{g, 1}\right) \subset W_{i} \phi_{g, 1}, \quad \operatorname{Var}\left(W_{i} \phi_{g, 1}\right) \subset\left(W_{i-2} \psi_{g, 1}\right)(-1), \\
& G r_{i}^{W} \psi_{g}(M, F, K), \quad G r_{i}^{W} \phi_{g, 1}(M, F, K) \in M H(X, k, i),
\end{aligned}
$$

où $W$ est décalée par $n-1$ ou $n$ comme dans (5.1.6.2), et que $G r^{W p} \psi_{g} S \circ\left(i d \otimes N^{i}\right)$ et $G r^{W p} \phi_{g, 1} S \circ\left(i d \otimes N^{i}\right)$ sont des polarisations sur $P_{N} G r_{n-1+i}^{W} \psi_{g}(M, F, K)$ et $P_{N} G r_{n+i}^{W} \phi_{g, 1}(M, F, K)$, alors la condition (5.1.4.2) est valable pour $(M, F, K)$ et $g$, donc on a la décomposition (5.1.4.3) pour $(M, F, K)$ de sorte que $\left(M_{2}, F, K_{2}\right)$ dans (5.1.4.3) est canoniquement isomorphe à un facteur direct de $P_{N} G r_{n}^{W} \phi_{g, 1}$ $(M, F, K)$ et que la restriction de $S$ à $K_{2}$ s'identifie à la restriction de $G r^{W{ }^{p}} \phi_{g, 1} S$; en particulier $\left(M_{2}, F, K_{2}\right) \in M H(X, k, n)$ et $\left.S\right|_{K_{2}}$ est une polarisation.

Preuve. D'après 3.2.6, 5.1.4, 5.2.4 et 5.2.8, il suffit de vérifier la condition (5.1. 4.2), mais c'est équivalente à:

$$
G r^{W} \phi_{g, 1}=\operatorname{Im} G r^{W} \operatorname{can} \oplus \operatorname{Ker} G r^{W} \text { Var }
$$

d'après 5.1.14 (et par l'hypothèse de la proposition), donc l'assertion est réduite 
au lemme suivant d'après $5.1 .10-11$ et 5.2.12.

5.2.15. Lemme (comparer à 4.2.4). Soient $H^{i}$ (resp. $\left.H^{\prime i}\right)$ des k-structures de Hodge de poids $n-1+i$ (resp. $n+i)$. Soient

$$
\text { can }: H^{i} \rightarrow H^{\prime i-1} \text { et } \operatorname{Var}: H^{\prime i} \rightarrow H^{i-1}(-1)
$$

des morphismes de structures de Hodge tels que $N^{i}: H^{i} \rightarrow H^{-i}(-i)$ et $N^{i}: H^{\prime i} \rightarrow$ $H^{\prime-i}(-i)$ sont des isomorphismes, où $N=$ Varocan ou can॰Var. Soient $S: H^{i} \otimes$ $H^{-i} \rightarrow \mathbb{k}(1-n), S^{\prime}: H^{\prime i} \otimes H^{\prime-i} \rightarrow \mathbb{k}(-n)$ des accouplements des structures de Hodge $(i \in \mathbb{Z})$ tels que

$$
\begin{aligned}
& \left.S \circ(N \otimes i d)+S \circ(i d \otimes N)=0 \quad \text { (de même pour } S^{\prime}\right) \\
& S^{\prime} \circ(\operatorname{can} \otimes i d)=S \circ(i d \otimes V a r)
\end{aligned}
$$

et que $S \circ\left(i d \otimes N^{i}\right)$ est une polarisation de structures de Hodge [6] sur la partie primitive $P_{N} H^{i}$ pour $i \geqq 0$ (de même pour $S^{\prime}$ ), on a alors

$$
S^{\prime}=\operatorname{Im} \text { can } \oplus \text { Ker Var . }
$$

Preuve. Notons que can: $H^{i} \rightarrow H^{\prime i-1}$ et Var: $H^{\prime i} \rightarrow H^{i-1}(-1)$ sont injectifs pour $i \geqq 1$, et que

$$
\begin{aligned}
& \operatorname{can}\left(P H^{i}\right) \subset P H^{i-1} \oplus N\left(P H^{i+1}(1)\right) \\
& \operatorname{Var}\left(P H^{\prime i}\right) \subset P H^{i-1}(-1) \oplus N\left(P H^{i+1}\right), \quad \text { cf. } 4.1 .2
\end{aligned}
$$

alors il suffit de vérifier:

$$
\begin{array}{ll}
\operatorname{can}\left(P H^{i}\right) \subset P H^{i-1} & \text { pour tout } i, \\
\operatorname{can}\left(P H^{i}\right)=P H^{\prime i-1} & \text { pour } i \geqq 2,
\end{array}
$$

car ces conditions entraînent que can et Var sont compatibles avec la décomposition de Lefschetz, i.e.

$$
\bigoplus_{j} N^{j} P H^{i} \underset{\text { Var }}{\stackrel{\text { can }}{\rightleftarrows}} \bigoplus_{j} N^{j} P H^{i-1}
$$

En effet, pour $i=1$ on a

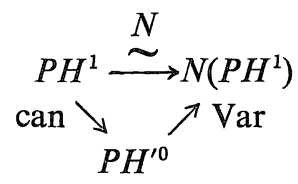

et pour $i=0, P H^{0} \subset \mathrm{Ker}$ can d'après (5.2.15.1).

D'abord on voit que (5.2.15.2) pour $i=j+2$ entraîne (5.2.15.1) pour $i=j$. 
En effet, pour $u \in P H^{j}$, posons can $u=u_{0}+N u_{1}$ avec $u_{k} \in P H^{j-1+2 k}$, alors $u_{1}=$ can $u_{1}^{\prime}$ pour $u_{1}^{\prime} \in P H^{j+2}$ par hypothèses. Puisque

$$
N u=\operatorname{Var} u_{0}+N^{2} u_{1}^{\prime}=v_{0}+N v_{1}+N^{2} u_{1}^{\prime}
$$

avec $v_{k} \in P H^{i-2+2 k}$, on a $u=v_{1}$ et $v_{0}=u_{1}^{\prime}=u_{1}=0$; d'où (5.2.15.1) pour $i=j$.

Si $i \gg 0$ on a $P H^{i}=0$, dans ce cas il faut montrer que $P H^{\prime i-1}=0$; mais cela résulte de la positivité de polarisation, car on a

$$
S \circ\left(\operatorname{Var} \otimes N^{i-2} \operatorname{Var}\right)+S^{\prime} \circ\left(i d \otimes N^{i-1}\right)=0
$$

sur $H^{\prime i-1} \otimes H^{\prime i-1}$ (i.e. si $u \in P H^{\prime i-1}$ et $\operatorname{Var} u \in P H^{i-2}(i \geqq 2)$, on a $u=0$.) Donc, par l'injectivité de Var ci-dessus, il suffit de montrer:

$$
\operatorname{Var}\left(P H^{\prime i-1}\right) \subset N\left(P H^{i}\right) \quad \text { pour } i \geqq 2
$$

en supposant (5.2.15.1) pour le même $i$. Pour $u \in P H^{\prime i-1}$, posons $\operatorname{Var} u=u_{0}+$ $N u_{1}$ avec $u_{k} \in H^{i-2+2 k}$. Si $u_{0} \neq 0$, on a $u^{\prime}:=u-\operatorname{can} u_{1} \in P H^{\prime i-1}$ tel que $\operatorname{Var} u^{\prime} \in$ $P H^{i-2}$ et que $u^{\prime} \neq 0$; mais c'est contradictoire à la positivité ci-dessus; d'où l'assertion. Ce qui achève la preuve de 5.2.14.

5.2.16. Proposition. Pour une variété complexe lisse $X$, on définit $K_{X}=$ $\boldsymbol{k}_{X}\left[d_{X}\right]$ et $S_{X}: K_{X} \otimes K_{X} \rightarrow a_{X}^{1} \boldsymbol{k}\left(-d_{X}\right)$ par

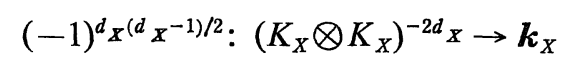

comme dans 5.2 .12 , alors $S_{X}$ est $(-1)^{d} x$-symétrique et

$$
\left(K_{X \times Y}, S_{X \times Y}\right) \cong\left(K_{X}, S_{X}\right) \bigotimes\left(K_{Y}, S_{Y}\right)
$$

i.e. $K_{X \times Y} \cong K_{X} \bigotimes K_{Y}$ et le diagramme suivant commute (cf. [11]):

$$
\begin{gathered}
K_{X \times Y} \otimes K_{X \times Y} \stackrel{S_{X \times Y}}{\longrightarrow} a_{X \times Y}^{!} \boldsymbol{k}\left(-d_{X}-d_{Y}\right) \\
\mid R \\
\left(K_{X} \otimes K_{X}\right) \bigotimes\left(K_{Y} \otimes K_{Y}\right) \stackrel{S_{X} \bigotimes S_{Y}}{\longrightarrow} a_{X}^{!} \boldsymbol{k}\left(-d_{X}\right) \bigotimes a_{Y}^{\prime} \boldsymbol{k}\left(-d_{Y}\right)
\end{gathered}
$$

Soit $g$ une fonction holomorphe sur $X$ telle que $D=g^{-1}(0)$ est un diviseur réduit à croisements normaux. Soit $W$ la filtration de ${ }^{p} \psi_{g} K_{X}$ associée à $N$ décalée par $d_{X}-1$ comme dans 5.1.6, alors la restriction de ${ }^{p} \psi_{g} S_{X} \circ\left(i d \otimes N^{i}\right)$ à la partie primitive de $\mathrm{Gr}_{d_{X^{-1+i}}}^{W}(i)$ coïncide à $\left(a_{i+1}\right)_{*} S_{\widetilde{D}^{(i+1)}}$ à un constant multiplicatif positif près par l'isomorphisme canonique:

$$
P_{N} G r_{X^{-1+i}}^{W} \psi_{g} K_{X}(i) \cong\left(a_{i+1}\right)_{*} K_{\widetilde{D}^{(i+1)}} \otimes \varepsilon^{i+1}
$$

cf. 3.6.1 pour la notation. 
Preuve. La première assertion est claire, car

$$
\left(d_{X}+d_{Y}\right)\left(d_{X}+d_{Y}-1\right) / 2=d_{X}\left(d_{X}-1\right) / 2+d_{Y}\left(d_{Y}-1\right) / 2+d_{X} d_{Y}
$$

D'après 3.4 .12 et 3.6 .10 , on a un isomorphisme canonique:

$$
P_{N} G r_{X^{-1+i}}^{W} \psi_{g}\left(K_{X} \otimes C\right) \cong\left(a_{i+1}\right)_{*} C_{\widetilde{D}^{(i+1)}}\left[d_{X}-i-1\right] \otimes \varepsilon^{i+1}
$$

(en particulier, $\left.{ }^{p} \psi_{g, 1}={ }^{p} \psi_{g}\right)$. D'après 5.1.12, can: $\left(\psi_{g, 1}, W\right) \rightarrow\left(\phi_{g, 1}, W\right)$ est strict et

$$
\operatorname{Ker}\left(G r_{d^{-1-i}}^{W} \text { can }\right) \cong N^{i}\left(P_{N} G r_{d^{-1+i}}^{W} \psi_{g, 1}\right)(i)
$$

par la décomposition de Lefschetz. D'autre part, on a une suite exacte:

$$
0 \rightarrow K_{D} \rightarrow{ }^{p} \psi_{g, 1} K \rightarrow{ }^{p} \phi_{g, 1} K \rightarrow 0
$$

où $K_{D}=\mathbb{k}_{D}\left[d_{X}-1\right]$, et une résolution canonique de $K_{D}$ par le complexe:

$$
\left[\left(a_{1}\right)_{*} \mathbb{R}_{\tilde{D}}^{(1)} \otimes \varepsilon^{1} \rightarrow \cdots \rightarrow\left(a_{d_{X}}\right)_{*} \mathbb{E}_{\tilde{D}}\left(d_{X}\right) \otimes \varepsilon^{d} x\right]
$$

muni d'une filtration $W$ définie par la filtration bête, i.e. $W_{i}=\sigma_{\geqq-i}$. Alors $W$ est la unique filtration de $K_{D}$ dans $\operatorname{Perv}\left(k_{X}\right)$, telle que $G r_{d_{X^{-i}}}^{W}$ soit le plus grand sous-objet de $K_{D} / W_{d_{X^{-i-1}}}$, à support dans $D^{(i)}$ pour tout $i$. D'après (5.2.16.2-3), on vérifie par récurrence que cette filtration $W$ coïncide à la filtration induite par la filtration $W$ de $\psi_{g, 1} K$ par la suite exacte (5.2.16.4); d'où (5.2.16.1).

On démontre 1'assertion pour la polarisation par récurrence sur $i$. Puisque l'assertion est locale et compatible avec le produit $\bigotimes$, on peut supposer que $X$ est un polydisque et que $g=x_{1} \cdots x_{n}$ avec $n=d_{X}=i+1$. On a alors la trivialisation: $\varepsilon^{j} \cong \mathbb{Z}$ pour $j \geqq 1$ par l'ordre des coordonnées. Le cas $i=0$ est clair par définition (cf. la preuve de 5.2.12). Supposons que $i>0$. Soient $\bar{n}=$ $\{1, \cdots, n\}$ et $\mathbb{I}$ un sous-ensemble de $\bar{n}$. On définit:

$$
\left.\Psi_{I}=\Psi_{x_{1}} \ldots \Psi_{x_{n}}^{p} \psi_{g} K_{X} \text {, où } \Psi_{x_{j}}={ }^{p} \phi_{x_{j, 1}} \text { (si } j \in I\right) \text { et }{ }^{p} \psi_{x_{j, 1}} \text { (sinon), }
$$

on a alors les morphismes:

$$
\operatorname{can}_{j}: \Psi_{I} \rightarrow \Psi_{I \cup\{j\}} \quad(j \notin I) \quad \text { et } \quad \operatorname{Var}_{j}: \Psi_{I} \rightarrow \Psi_{I \backslash j\}}(-1) \quad(j \in I)
$$

induits par les foncteurs ${ }^{p} \psi_{x_{j, 1}}$ et ${ }^{p} \phi_{x_{j, 1}}$, et la filtration $W$ de $\psi_{I}$ induite par celle de ${ }^{p} \psi_{g} K_{X}$, car les foncteurs $\psi, \phi$ sont exacts. On a de plus les accouplements:

$$
S_{I}: \Psi_{I} \otimes \Psi_{I} \rightarrow \mathbb{R}(1-|\mathbb{I}|)
$$

d'après 5.2.3-4. Donc il suffit de vérifier que l'image de 


$$
S_{\bar{n}} \circ\left(i d \otimes N^{i}\right): G r_{2 i}^{W} \Psi_{\bar{n}}(i) \otimes G r_{2 i}^{W} \Psi_{\bar{n}}(i) \rightarrow \boldsymbol{k}
$$

est non nulle et contenue dans $\boldsymbol{R}_{\geq 0}$ (où $i=d_{X}-1$ ) d'après 5.2.8. Soit

$$
\widetilde{K}={ }^{p} \phi_{x_{2}, 1} \cdots{ }^{p} \phi_{x_{n}, 1} \psi_{g} K_{X} \in \operatorname{Perv}\left(k_{c}\right)
$$

où $C=\left\{x_{2}=\cdots=x_{n}=0\right\}$, alors ${ }^{p} \psi_{x_{1}, 1} \tilde{K}=\Psi_{\bar{n} \backslash\{1}$ et ${ }^{p} \phi_{x_{1}, 1} \tilde{K}=\Psi_{\bar{n}}$. (Notons que $\Psi_{\bar{n}} \cong \boldsymbol{k}[N] /\left(N^{i+1}\right)$ et $\Psi_{\bar{n} \backslash\{1} \cong \boldsymbol{k}[N] /\left(N^{i}\right)$ comme $\boldsymbol{k}[N]$-module (non-canoniquement) d'après 3.6.8, où $N$ est toujours associé au foncteur ${ }^{p} \psi_{g, 1}$.) Soit

$$
\tilde{S}: \tilde{K} \otimes \tilde{K} \rightarrow \mathbb{k}(-i)
$$

l'accouplement induit, alors ${ }^{p} \psi_{x_{1}, 1} \tilde{S}=S_{\bar{n} \backslash\{1\}}$ et ${ }^{p} \phi_{x_{1}, 1} \tilde{S}=S_{\bar{n}}$. Par hypothèse de récurrence,

$$
\tilde{S \circ}\left(i d \otimes N^{i-1}\right): G r_{2 i-1}^{W} \widetilde{K}(i-1) \otimes G r_{2 i-1}^{W} \widetilde{K}(i-1) \rightarrow a_{c}^{!} k(-1)
$$

coïncide à $S_{C}: K_{C} \otimes K_{C} \rightarrow a_{C}^{!} \mathbb{k}(-1)$ à un constant multiplicatif positif près (car $G r^{W}$ commute à $\phi_{x_{j}}$ ), donc l'image de

$$
S_{\bar{n} \backslash\{1\}} \circ\left(i d \otimes N^{i-1}\right): G r_{2 i-1}^{W} \Psi_{\bar{n} \backslash\{1\}}(i-1) \otimes G r_{2 i-1}^{W} \Psi_{\bar{n} \backslash\{1\}}(i-1) \rightarrow k
$$

est non nulle et contenue dans $\boldsymbol{R}_{\geqq 0}$. D'après 3.4 .12 et 3.6 .8 , on a

et

$$
\begin{aligned}
& \operatorname{Var}_{1}: G r_{2 i}^{W} \Psi_{\bar{n}} \underset{\rightarrow}{\rightarrow} G r_{2 i-1}^{W} \Psi_{\bar{n} \backslash\{1\}}(-1) \\
& N^{i-1}: G r_{2 i-1}^{W} \Psi_{\bar{n} \backslash\{1\}} \stackrel{G}{\rightarrow} G r_{1}^{W} \Psi_{\bar{n} \backslash\{1}(1-i) \\
& N=-\operatorname{can}_{1} \operatorname{Var}_{1}\left(\operatorname{car}(d x \otimes 1)\left(x_{1} \partial_{1}+s+1\right)=0\right) .
\end{aligned}
$$

D'après 5.2 .4 , on a

$$
S_{\bar{n}} \circ\left(\operatorname{can}_{1} \otimes i d\right)=S_{\bar{n} \backslash\{1\}} \circ\left(i d \otimes \operatorname{Var}_{1}\right): G r_{1}^{W} \Psi_{\bar{n} \backslash\{1\}} \otimes G r_{2 i}^{W} \Psi_{\bar{n}} \rightarrow k(-i),
$$

donc

$$
S_{\bar{n}} \circ\left(i d \otimes N^{i}\right)=S_{\bar{n} \backslash[1]} \circ\left(\operatorname{Var}_{1} \otimes N^{i-1} \operatorname{Var}_{1}\right): G r_{2 i}^{W} \Psi_{\bar{n}} \otimes G r_{2 i}^{W} \Psi_{\bar{n}} \rightarrow \boldsymbol{k}(-2 i),
$$

$\operatorname{car} N^{i-1} \operatorname{Var}_{1}=\operatorname{Var}_{1} N^{i-1}$ et $\widetilde{S}(N \otimes i d)=-\tilde{S}(i d \otimes N)$; d'où l'assertion.

5.2.17. Soit $\mathcal{C}$ une catégorie triangulée. Soit $\boldsymbol{A}=\left\{(p, q) \in(\boldsymbol{Z} \cup\{ \pm \infty\})^{2}\right.$ : $p \leqq q\}$. Pour $\alpha=(p, q)$, posons $\alpha_{1}=p$ et $\alpha_{2}=q$. On définit un ordre de $\Lambda$ par: $\alpha>\beta$ sii $\alpha_{i} \geqq \beta_{i}$ pour $i=1,2$. Alors un objet quasi-filtré de $\mathcal{C}$ consiste en des objets $K(\alpha) \operatorname{de} C$ pour $\alpha \in \Lambda$, munis de morphismes:

$$
\begin{array}{lll}
u_{\beta \alpha}: K(\alpha) \rightarrow K(\beta) & \text { pour } & \alpha>\beta \\
v_{\beta \alpha}: K(\alpha) \rightarrow K(\beta)[1] & \text { pour } & \alpha_{2}=\beta_{1}
\end{array}
$$


satisfaisant les conditions:

$$
\begin{aligned}
& u_{\alpha \infty}=i d \quad \text { pour } \quad \alpha \in \Lambda \\
& u_{\gamma_{\alpha}}=u_{\gamma_{\beta}} u_{\beta \alpha} \quad \text { pour } \quad \alpha>\beta>\gamma \\
& u_{\delta \beta} v_{\beta \alpha}=v_{\delta \gamma} u_{\gamma_{\alpha}} \quad \text { pour } \alpha>\gamma, \beta>\delta \text {, } \\
& \alpha_{2}=\beta_{1}, \gamma_{2}=\delta_{1}
\end{aligned}
$$

(5.2.17.2) il existe $n, m \in \mathbb{Z}$ tels que $K(\alpha)=0$ si $\alpha_{2} \leqq n$ ou $\alpha_{1} \geqq m$.

(5.2.17.3) pour $\alpha, \beta, \gamma \in \Lambda$ tels que $\alpha_{1}=\gamma_{2}, \alpha_{2}=\beta_{2}, \beta_{1}=\gamma_{1}$,

$$
K(\alpha) \stackrel{u_{\beta \alpha}}{\longrightarrow} K(\beta) \stackrel{u_{\gamma \beta}}{\longrightarrow} K(\gamma) \stackrel{v_{\alpha \gamma}}{\longrightarrow} K(\alpha)[1]
$$

est un triangle distingué de $\mathcal{C}$.

Posons $K(p /)=K(p,+\infty), K=K(-\infty,+\infty), K(/ p)=K(-\infty, p), K(p / q)=K(p, q)$ et $G r^{p} K=K(p / p+1)$, on a alors

$$
K(p /) \cong K \quad \text { si } p \leqq n \text { et nul si } p \geqq m .
$$

Pour $-\infty \leqq p<q<r<s \leqq+\infty$ on a un diagramme de l'octaèdre:

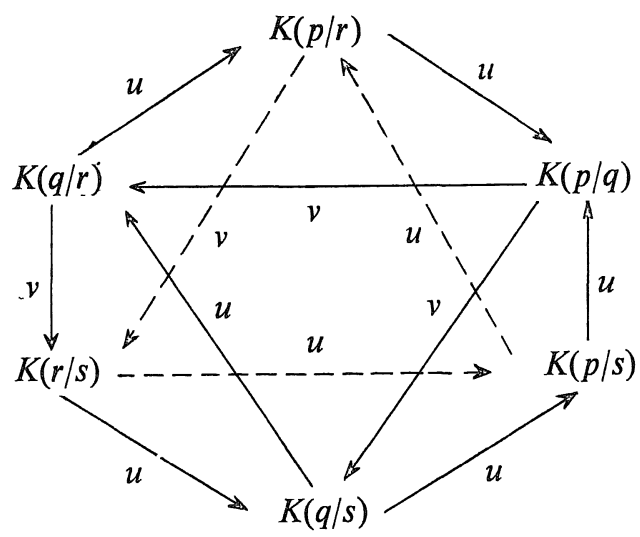

Notons que les objets quasi-filtrés sont stables par foncteurs exacts de catégories triangulées. Si $\mathcal{C}=D_{c}^{b}\left(\boldsymbol{k}_{X}\right)$ (resp. $\mathcal{C}$ est la catégorie dérivée d'une catégorie abélienne), un faisceau pervers (resp. un complexe) $K$ muni d'une filtration décroissante finie $F$ définit uniquement un objet quasi-filtré $\{K(\alpha) ; u, v\}$ tel que $K(p / q) \cong F^{p} K / F^{q} K$ d'après [1]. De même, si $C$ est une $t$-catégorie on associe à un objet $K$ de $\mathcal{C}^{\geqq a} \cap \mathcal{C}^{\leqq b}$ un objet quasi-filtré $\{K(\alpha) ; u, v\}$ tel que $K(p / q)=$ $\tau_{\leqq-p} \tau_{>-q} K$, cf. [loc. cit]. Alors la suite spectrale de Leray (à un décalage près) est associée à (l'image par un foncteur exact de) cet objet quasi-filtré par le lemme ci-dessous (cf. [6]). Notons que si $\mathcal{C}=D_{c}^{b}\left(\boldsymbol{k}_{X}\right)$, la $t$-structure perverse est 
auto-duale [1].

5.2.18. Lemme. (Verdier). Avec les notations de 5.2.17, soient $\mathcal{A}$ une catégorie abélienne et $H^{i}: \mathcal{C} \rightarrow \mathcal{A}(i \in Z)$ un foncteur cohomologique. Alors on a une suite spectrale dans $\mathcal{A}$ :

$$
E_{1}^{p q}=H^{p+q} G r^{p} K \Rightarrow H^{p+q} K
$$

telle que la filtration $F$ de $H^{i} K$ soit définie par

$$
F^{p} H^{i} K=\operatorname{Im}\left(H^{i} K(p /) \rightarrow H^{i} K\right)=\operatorname{Ker}\left(H^{i} K \rightarrow H^{i} K(/ p)\right) .
$$

Preuve (d'après [37]). On définit pour $p, q, i \in \boldsymbol{Z}$ tels que $p+q=i$ et pour $1 \leqq r \leqq \infty$ :

$$
\begin{aligned}
Z_{r}^{p q} & =\operatorname{Im}\left(H^{i} K(p / p+r) \stackrel{u}{\rightarrow} H^{i} G r^{p} K\right) \\
& =\operatorname{Ker}\left(H^{i} G r^{p} K \stackrel{v}{\rightarrow} H^{i+1} K(p+1 / p+r)\right) \\
H^{i} G r^{p} K / B_{r}^{p q} & =\operatorname{Coim}\left(H^{i} G r^{p} K \stackrel{u}{\rightarrow} H^{i} K(p-r+1 / p+1)\right) \\
& =\operatorname{Coker}\left(H^{i-1}(p-r+1 / p) \stackrel{v}{\rightarrow} H^{i} G r^{p} K\right) \\
E_{r}^{p q} & =\operatorname{Im}\left(Z_{r}^{p q} \rightarrow H^{i} G r^{p} K / B_{r}^{p q}\right) .
\end{aligned}
$$

Notons que $Z_{r}^{p q} \supset B_{r}^{p q}$ car la composition:

$$
K(p / q) \stackrel{v}{\rightarrow} K(q / r)[1] \stackrel{v}{\rightarrow} K(r / s)[2]
$$

est nulle pour $p<q<r<s$ d'après (5.2.17.4). On a de plus les isomorphismes:

$$
\begin{aligned}
G r_{F}^{p} H^{i} K & \cong \operatorname{Im}\left(H^{i} K(p /) \stackrel{u}{\rightarrow} H^{i} K(/ p+1)\right) \cong E_{\infty}^{p q} \\
Z_{r}^{p q} / Z_{r+1}^{p q} & \cong \operatorname{Im}\left(H^{i} K(p / p+r) \stackrel{v u}{\rightarrow} H^{i+1}(p+1 / p+r+1)\right) \\
& \cong B_{r+1}^{p+r, q-r+1} / B_{r}^{p+r, q-r+1}
\end{aligned}
$$

par le diagramme de l'octaèdre (5.2.17.4) pour $(p, q, r, s)=(-\infty, p, p+1,+\infty)$, $(p, p+1, p+r, p+r+1)$ et par la commutativité (5.2.17.1). On définit les différentielles $d_{r}^{p q}: E_{r}^{p q} \rightarrow E_{r}^{p+r, q-r+1}$ par la composition:

$$
\begin{aligned}
E_{r}^{p q} & =Z_{r}^{p q} / B_{r}^{p q} \rightarrow Z_{r}^{p q} / Z_{r+1}^{p q} \cong B_{r+1}^{p+r, q-r+1} / B_{r}^{p+r, q-r+1} \\
& \hookrightarrow Z_{r}^{p+r, q-r+1} / B_{r}^{p+r, q-r+1}=E_{r}^{p+r, q-r+1} .
\end{aligned}
$$

On a alors $d_{r}^{p q} d_{r}^{p-r, q+r-1}=0$ et

$\operatorname{Ker} d_{r}^{p q} / \operatorname{Im} d_{r}^{p-r, q+r-1} \cong H\left(B_{r+1}^{p q} / B_{r}^{p q} \rightarrow Z_{r}^{p q} / B_{r}^{p q} \rightarrow Z_{r}^{p q} / Z_{r+1}^{p q}\right) \cong E_{r+1}^{p q}$, 
d'où 1'assertion.

5.2.19. Remarque. La construction de la suite spectrale (5.2.18.1) est autoduale (cf. [6]). En effet, on a l'objet quasi-filtré dual: $\{\stackrel{\circ}{K}(\alpha) ; \stackrel{\circ}{\text {, }}, \stackrel{\circ}{\}}\}$ par $\stackrel{\circ}{K}(p / q)=$ $K(-q+1 /-p+1)^{\circ} \in \mathcal{C}^{\circ}$, et $\stackrel{\circ}{H}^{i}: \mathcal{C}^{\circ} \rightarrow \mathcal{A}^{\circ}$ par $\stackrel{\circ}{H}^{i}=\left(H^{-i}\right)^{\circ}$; donc onobtient une suite spectrale dans $\mathcal{A}^{\circ}$ :

$$
\stackrel{\circ}{E}_{1}^{p q}=\stackrel{\circ}{H}^{p+q} G r^{p} \stackrel{\circ}{K} \Rightarrow \stackrel{\circ}{H}^{p+q} \stackrel{\circ}{K}
$$

en appliquant 5.2.18 à $\{\stackrel{\circ}{K}(\alpha) ; \stackrel{\circ}{u}, \stackrel{o}{v}\}$. Par la construction, (5.2.19.1) est canoniquement isomorphe à la suite spectrale duale de $(5.2 .18 .1)$, i.e. on a les isomorphismes canoniques:

$$
\stackrel{\circ}{E}_{r}^{p q} \cong\left(E_{r}^{-p,-q}\right)^{\circ}, \quad G r_{F}^{p} \stackrel{\circ}{H}^{i} \stackrel{\circ}{K} \cong\left(G r_{F}^{-p} H^{-i} K\right)^{\circ}
$$

compatibles avec les différentielles: $d_{r}^{p q}, \stackrel{\circ}{d}_{r}^{p q}$ et avec les isomorphismes: $E^{p q} \cong$ $G r_{F}^{p} H^{p+q} K$, etc.

5.2.20. Remarque. Si $\mathcal{C}=D_{c}^{b}\left(\boldsymbol{k}_{X}\right), \mathcal{A}=\operatorname{Perv}\left(\mathbb{k}_{X}\right)$ et $H^{0}$ est le foncteur cohomologique pervers (cf. [1]), on a le foncteur dual:

$$
\mathbb{D}: \mathcal{C}^{\circ} \rightarrow C, \mathbb{D}: \mathscr{A}^{\circ} \rightarrow \mathcal{A}
$$

compatible avec les foncteurs $\stackrel{\circ}{H}^{i}, H^{i}$. En appliquant 5.2.18 à $\left\{\mathbb{D} \dot{K}^{\circ}(\alpha) ; \mathbb{D} \dot{u}, \mathbb{D} \nu\right.$, , on obtient:

$$
\mathbb{D} \dot{E}_{1}^{p q}=H^{p+q} \mathbb{D} G r_{F}^{p} \stackrel{\circ}{K} \Rightarrow H^{p+q} \mathbb{D} \stackrel{\circ}{ }
$$

Cette suite spectrale est canoniquement isomorphe à (5.2.19.1) par le foncteur ID.

Soit $\left\{K^{\prime}(\alpha) ; u^{\prime}, v^{\prime}\right\}$ un objet quasi-filtré, et soit

$$
w:\left\{K^{\prime}(\alpha) ; u^{\prime}, v^{\prime}\right\} \rightarrow\{\mathbb{D} K(\alpha) ; \mathbb{D} \dot{0}, \mathbb{D} \dot{v}\}
$$

un morphisme des objets quasi-filtrés, i.e. $w$ est une famille de morphismes $w_{\alpha}: K^{\prime}(\alpha) \rightarrow \stackrel{0}{K}(\alpha)$ compatibles avec $u^{\prime}, v^{\prime}$, etc. Alors on obtient un morphisme de la suite spectrale (5.2.18.1) pour $\left\{K^{\prime}(\alpha) ; u^{\prime}, v^{\prime}\right\}$ dans (5.2.20.1), i.e. on a des accouplements canoniques:

$$
\begin{aligned}
& E_{r}^{\prime p q} \otimes E_{r}^{-p,-q} \rightarrow a_{X}^{!} \not e \\
& \left(H^{i} K^{\prime}, F\right) \otimes\left(H^{-i} K, F\right) \rightarrow a_{X}^{!} k
\end{aligned}
$$

compatibles avec les différentielles $d_{r}^{\prime p q}, d_{r}^{p q}$ et avec les isomorphismes: $E_{\infty}^{\prime p q} \cong G r_{F}^{p} H^{p+q} K^{\prime}$, etc. 
5.2.21. Remarque. Si $\mathcal{C}=D(\mathcal{A})$ pour une catégorie abélienne $\mathcal{A},\left\{K^{*} ; u, v\right\}$ est associé à un complexe filtré $\left(K^{\circ}, F\right)$ (cf. 5.2.17) et $H$ est le foncteur cohomologique usuel, alors (5.2.18.1) coïncide à la suite spectrale usuelle associée à $\left(K^{*}, F\right)$ comme dans [6] [37].

\subsection{Stabilité par image directe par un morphisme projectif}

Dans ce paragraphe on démontre

5.3.1. Théorème. Soit $f: X \rightarrow Y$ un morphisme projectif de variétés complexes lisses, et soit l la première classe de Chern d'un faisceau inversible relativement ample pour $f$. Soit $(M, F, K) \in M H_{Z}(X, k, n)$ muni d'une polarisation $S$, alors

(5.3.1.1) $f_{*}(M, F)$ est strict et $\mathscr{H}^{i} f_{*}(M, F, K) \in M H(Y, k, n+i), \quad c f$. (5.1.3.2).

(5.3.1.2) $l^{i}: \mathscr{H}^{-i} f_{*}(M, F, K) \underset{\rightarrow}{\rightarrow} \mathscr{H}^{i} f_{*}(M, F, K)(i)$ pour $i \geq 0$

(Lefschetz vache),

(5.3.1.3) $\quad(-1)^{i(i-1) / 2 p} \mathscr{H} f_{*} S \circ\left(i d \otimes l^{i}\right): P_{l}^{p} \mathcal{H}^{-i} f_{*} K \otimes P_{l}^{p} \mathcal{H}^{-i} f_{*} K \rightarrow a_{Y}^{!} \mathbb{k}(-n+i)$ est une polarisation de la partie l-primitive $P_{l} \mathcal{H}^{-i} f_{*}(M, F, K)$ $\left(:=\operatorname{Ker} l^{i+1} \subset \mathcal{H}^{-i} f_{*}(M, F, K)\right)$ pour $i \geq 0$.

La démonstration est par récurrence $\operatorname{sur} \operatorname{dim} Z$ : le cas $\operatorname{dim} f(Z)>0$ sera traité en 5.3.4-6, et $\operatorname{dim} f(Z)=0$ en 5.3.8-11. D'abord on définit l'action de $l$ sur $\mathscr{H}^{*} f_{*}(M, F)$ (ou plutôt $\operatorname{sur}(M, F)$ dans $D F\left(\mathscr{D}_{X}\right)$ ).

5.3.2. Lemme. Soit $X$ une variété complexe lisse. Soit $\omega$ une forme $C^{\infty}$ fermée de type (1.1), on a alors un morphisme fonctoriel pour $(M, F) \in$ $\operatorname{DF}\left(\mathscr{D}_{X}\right)$ :

$$
\omega_{\wedge}:(M, F) \rightarrow(M, F)(1)[2]
$$

compatible avec l'action naturelle de $l=[\omega] \in H^{2}(X, C)$ :

$$
l: D R M \rightarrow D R M[2] \text { dans } D\left(\boldsymbol{C}_{X}\right) .
$$

Si de plus $\omega=d \pi$ avec $\pi$ une forme $C^{\infty}$ de type $(1,0)$, le morphisme (5.3.2.1) est nul.

Preuve. Soit ${ }^{\infty} \Omega_{X}^{*}$ le complexe double des formes $C^{\infty}$ complexes, on définit une filtration $F$ par:

$$
F_{p}^{\infty} \Omega_{X}^{i j}={ }^{\infty} \Omega_{X}^{i j} \text { si } p \geq-i \text { et } 0 \text { sinon. }
$$

On a alors un quasi-isomorphisme filtré: $\left(\Omega_{X}^{\circ}, F\right) \rightarrow\left({ }^{\infty} \Omega_{X}^{\circ}, F\right)$ par le lemme de 
Dolbeault. Soit $d=\operatorname{dim} X$, on a alors des quasi-isomorphismes filtrés:

$$
\left({ }^{\infty} \Omega_{X}^{*}, F\right) \otimes_{\mathcal{O}}\left(\mathscr{D}_{X}, F\right)[d] \leftarrow\left(\Omega_{X}^{\circ}, F\right) \otimes_{\mathcal{O}}\left(\mathscr{D}_{X}, F\right)[d] \rightarrow\left(\Omega_{X}^{d}, F\right)
$$

Soit $(L, F)$ le premier complexe de (5.3.2.2), alors on obtient

$$
\omega_{\wedge}:(L, F) \rightarrow(L, F)(1)[2]
$$

et une homotopie de $\omega_{\wedge}$ :

$$
h=\pi_{\wedge}:(L, F) \rightarrow(L, F)(1)[1]
$$

(i.e. $d h+h d=\omega_{\wedge}$ ) si $\pi$ est comme ci-dessus. Donc pour définir (5.3.2.1) (et son homotopie si $\omega=d \pi$, etc.)., il suffit de vérifier que les morphismes dans (5.3.2.2), tensorisés par un $\mathscr{D}_{X}$-Module à gauche filtré $(M, F) \otimes_{\mathcal{O}}\left(\Omega_{X}^{d}, F\right)^{-1}$ sont des quasiisomorphismes filtrés; mais cela résulte du fait que ${ }^{\infty} \Omega_{X}^{i j}$ sont plats sur $\mathcal{O}_{X}$, cf. [39], et de 2.1.6. Il reste à démontrer la compatibilité avec l'action de $l$. Par définition, l'action de $l$ s'écrit:

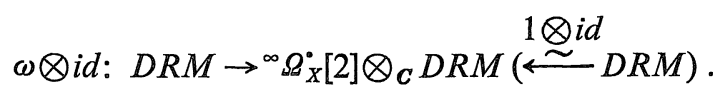

Posons

$$
K:={ }^{\infty} \Omega_{X}^{\cdot} \otimes_{\mathcal{O}}\left(M \otimes\left(\Omega_{X}^{d}\right)^{-1}\right)\left(=D R\left(L \otimes_{\mathcal{O}}\left(M \otimes\left(\Omega_{X}^{d}\right)^{-1}\right)\right) \simeq D R M\right)
$$

alors la composition de $1 \otimes i d: K \rightarrow{ }^{\infty} \Omega_{X}^{\circ} \otimes_{C} K$ et de $\wedge:{ }^{\infty} \Omega_{X}^{\circ} \otimes_{C} K \rightarrow K$ est identique, i.e. $\wedge$ est l'inverse de $1 \otimes i d$; d'où l'assertion.

5.3.3. Remarque. Soit $L$ un faisceau inversible, i.e. un fibré véctoriel de rang 1. Soit $u$ une métrique hermitienne de $L$. Si $L$ est représenté par un 1-cycle $\left\{f_{\alpha \beta}\right\}$ associé à un recouvrement $\left\{U_{\alpha}\right\}$ de $X, u$ est représenté par $\left\{u_{\alpha}\right\}$ tel que $u_{\alpha}=\left|f_{\alpha \beta}\right|^{-2} u_{\beta}$, où $u_{\alpha}$ est une fonction $C^{\infty}$ réele sur $U_{\alpha}$. Alors c'est bien connu que $c_{1}(L)$ est représentée par une forme $\omega$ telle que $\omega=\partial \bar{\partial} \log u_{\alpha}$ sur $U_{\alpha}$. Si on change la métrique $u$, la différence de $\omega$ est donnée par $\partial \partial \bar{\partial} \log g$, où $g$ est une fonction $C^{\infty}$ réelle non nulle.

Preuve de 5.3.1 (dans le cas $\operatorname{dim} f(Z)>0$ ).

D'abord on réduit l'assertion à:

5.3.4. Proposition. Soient $f: X \rightarrow Y$ et $l$ comme dans 5.3.1. Soit $g$ une fonction holomorphe sur $Y$, posons $h=g f$. Soit $(M, F, K) \in M F_{h}\left(\mathscr{D}_{X}, k\right)$ à support strict $Z$ (cf. 5.1.3) tel que $h^{-1}(0) \nsupseteq Z$, que $(M, F)$ soit quasi-unipotent et régulier le long de $h$, et que $G r_{i}^{W} \psi_{h}(M, F, K), G r_{i}^{W} \phi_{h, 1}(M, F, K) \in M H(X, \mathbb{R}, i)$, où $W$ est décalée par $n-1$ ou $n$. Soit $S: K \otimes K \rightarrow a_{X}^{!} k(-n)$ un accouplement. Supposons que les conclusions de 5.3.1 soient vérifiées pour $f, l, P_{N} G r_{n-1+i}^{W} \psi_{h}(M, F, K)$ et $G r^{W} \psi_{h} S \circ\left(i d \otimes N^{i}\right)$ pour $i \geq 0$, alors 
(5.3.4.1) $f_{*}(M, F)$ est strict dans un voisinage de $g^{-1}(0), \mathscr{H}^{i} f_{*}(M, F)$ sont quasi-unipotents et réguliers le long de $\mathrm{g}$,

(5.3.4.2) la filtration $W$ de $\psi_{g} \mathscr{H}^{j} f_{*}(M, F, K) \simeq \mathscr{H}^{j} f_{*} \psi_{h}(M, F, K)$ induite par la filtration $W[j]$ de $\psi_{h}(M, F, K)$ est la filtration associée à $N$ décalée par $n-1+j(c f .1 .3 .9)$ telle que $G r_{i}^{W} \psi_{g} \mathscr{H}^{j} f_{*}(M, F, K) \in M H(Y, \boldsymbol{k}, i)$ (de même pour $\phi_{1}$, en remplacant $n-1$ par $n$ ).

(5.3.4.3) $l^{i}: \mathscr{H}^{-j} f_{*}(M, F, K) \rightarrow \mathcal{H}^{j} f_{*}(M, F, K)(i)$ est un isomorphisme au voisinage de $g^{-1}(0)(j \geq 0)$, $(-1)^{j(j-1) / 2} G r^{W p} \psi_{g}^{p} \mathscr{H} f_{*} S \circ\left(i d \otimes N^{i} l^{j}\right)$ est une polarisation sur la partie bi-primitive $P_{l} P_{N} G r_{n-1-j+i}^{W} \psi_{g} \mathcal{H}^{-j} f_{*}(M, F, K)\left(:=\operatorname{Ker} l^{j+1} \cap\right.$ $\operatorname{Ker} N^{i+1}$ ) pour $i, j \geq 0$ (de même pour $\phi_{g, 1}$ en remplaçant $n-1$ par $n$ ).

5.3.4 $\Rightarrow 5.3 .1$ (dans le cas $\operatorname{dim} f(Z)>0$ )

Puisque l'assertion est locale, on peut supposer que $f(Z)$ soit irréductible. Par hypothèse de récurrence, toutes les conditions de 5.3.4 sont satisfaites. On a donc (5.3.1.2) d'après (5.3.4.3) et $f_{*}(M, F)$ est strict d'après (5.3.4.1). Alors l'accouplement ${ }^{p} \mathscr{H} f_{*} S \circ\left(i d \otimes l^{i}\right)$ de ${ }^{p} \mathscr{H}^{-i} f_{*} K$ est compatible avec la filtration $F$ (cf. 5.2.9) d'après 2.5.6. On va vérifier que $\mathcal{H}^{j} f_{*}(M, F, K)$ sont quasiunipotents et réguliers le long de toute fonction holomorphe $g$ localement définie sur $Y$. Si $g^{-1}(0) \triangleright f(Z)$, l'assertion résulte de (5.3.4.1). $\quad$ Si $g^{-1}(0) \supset f(Z)$, elle est équivalente à l'isomorphisme:

$$
i_{g *} \mathcal{H}^{j} f_{*}(M, F) \simeq i_{0 *} \mathcal{H}^{j} f_{*}(M, F)
$$

d'après 3.2.6, où $i_{0}: Y=Y \times\{0\} \rightarrow Y \times C$. Mais c'est valable pour $(M, F)$ et pour $h$, donc cela résulte de la commutativité $(f \times i d) \circ i_{h}=i_{g} \circ f$ et du fait que $f_{*}(M, F)$ est strict.

D'après (5.3.4.1-2), (5.3.4.4) et 5.1.12, toutes les conditions de 5.2.14 sont satisfaites pour $P_{l} \mathcal{H}^{-j} f_{*}(M, F, K)$ et $(-1)^{j(j-1) / 2} p \mathcal{H} f_{*} S \circ\left(i d \otimes l^{j}\right)$ (car can, Var et $N$ sont compatibles avec l'image directe $f_{*}$ ); on a donc (5.3.1.1) et (5.3.1.3) d'après 5.1.5, 5.1.8 et (5.2.10.2), car la décomposition de Lefschetz (pour l'action de $l$ ) est compatible avec la filtration de Hodge $F$ d'après (5.3.1.2); d'où 5.3.1 (dans le cas $\operatorname{dim} f(Z)>0$ ).

Preuve de 5.3.4.

Soit $\tilde{f}=f \times i d: X \times C \rightarrow Y \times C$. Soient $i_{g}$ et $i_{h}$ comme dans 3.2.1. alors $\tilde{f} \circ i_{h}=i_{g} \circ f$. Posons $(\tilde{M}, F)=i_{h *}(M, F)$. Si $G r^{V} \tilde{f}_{*}(\tilde{M}, F)\left(\simeq f_{*} G r^{V}(\tilde{M}, F)\right)$ est strict, on obtient (5.3.4.1) et l'isomorphisme 


$$
\left.\psi_{g} \mathscr{H}^{j} f_{*}(M, F, K) \simeq \mathscr{H}^{j} f_{*} \psi_{h}(M, F, K) \text { (de même pour } \phi_{1}\right)
$$

en appliquant 3.3 .17 à $(\tilde{M}, F)$ et $\tilde{f}$. On a de même (5.3.4.3), si $G r^{V} l^{i}$ sont des isomorphismes. Donc l'assertion résulte de la proposition 5.3.5 ci-dessous (appliquée à $\psi_{h}(M, F, K)$, etc.); en fait $f_{*} \psi_{h}(M, F)$ est strict d'après 1.3.5-6 (appliqué à la projection de (5.3.5.1) dans $M G_{h}(\mathscr{B})$ ) et d'après 5.2.21, 5.1.14 (de même pour $\left.\phi_{1}\right)$, et (5.3.4.4) résulte de (5.3.5.3), 5.2.13, 5.2.7, 5.2.9 et de 5.2.19, car $(-1)^{j(j-1) / 2 p} \mathcal{H} f_{*} G r^{W}{ }^{p} \psi_{h} S \circ\left(i d \otimes N^{i} l^{j}\right)$ est une polarisation de $P_{N} P_{l 1} C_{i}^{-j}$ par hypothèse.

5.3.5. Proposition. Soient $f$ et $l$ comme ci-dessus. Soit $(M, F, K, W) \in$ $M H W(X, \mathbb{R})(c f .5 .1 .14)$ muni d'un morphisme

$$
N:(M, F, K, W) \rightarrow(M, F, K, W)(-1) \quad(=(M(-1), F[-1], K(-1), W[2]))
$$

tel que $N^{j}: G r_{r+j}^{W} \underset{\rightarrow}{\rightarrow} G r_{r-j}^{W}(-j)$. Soit $S: K \otimes K \rightarrow a_{X}^{!} \mathbb{k}(-r)$ un accouplement tel que $S(i d \otimes N)+S(N \otimes i d)=0$. Supposons que les conclusion de 5.3.1 soient satisfaites pour $f, l, P_{N} G r_{r+i}^{W}(M, F, K)$ et $G r^{W} S \circ\left(i d \otimes N^{i}\right)$ pour $i \geq 0($ ò̀ $n=r+i)$. On a alors une suite spectrale dans $M G_{h}(\mathscr{B}, \mathbb{k})$ (cf. 5.1.1):

$$
E_{1}^{p q}=\mathscr{H}^{p+q} f_{*} G r_{-p}^{W}(M, F, K) \Rightarrow \mathcal{H}^{p+q} f_{*}(M, F, K)
$$

qui dégénère en $E_{2}$, et telle que $E_{k}^{p q} \in M H(X, \mathbb{k}, q)^{p}(c f .5 .2 .12)$ et que $d_{1}$ est strict. Posons ${ }_{k} C_{i}^{j}=E_{k}^{-i-r_{0} i+j+r}$, alors:

$$
\begin{aligned}
& l^{j}:{ }_{k} C_{i}^{-j} \stackrel{\sim}{\rightarrow}{ }_{k} C_{i}^{j}(j) \quad \text { pour } j \geq 0 \\
& N^{i}:{ }_{k} C_{i}^{j} \underset{{ }_{k}}{ } C_{-i}^{j}(-i) \text { pour } i \geq 0
\end{aligned}
$$

(5.3.5.3) le morphisme naturel: $\mathbb{K e r} d_{1} \cap P_{N} P_{l 1} C_{i}^{-j} \rightarrow P_{N} P_{l 2} C_{i}^{-j}$ est un épimorphisme pour $i, j \geq 0$, où $P_{N} P_{l k} C_{i}^{-j}$ est la partie biprimitive $\left(=\operatorname{Ker} l^{j+1} \cap \operatorname{Ker} N^{i+1}\right) d e{ }_{k} C_{i}^{-j}$.

Preuve. Puisque $\mathcal{H}^{j} f_{*} G r_{i}^{W}(M, F, K) \in M H(Y, \mathbb{R}, i+j)^{p}$ par hypothèse (et par la décomposition de Lefschetz), on obtient la suite spectrale (5.3.5.1), en appliquant 5.2.18 à $f_{*}((M, F), W)$ (cf. 2.1.12), $f_{*}(K, W)$ et $D R f_{*}(M, W) \simeq C \otimes$ $f_{*}(K, W)$, car $D R \circ \mathscr{H}^{j}={ }^{p} \mathcal{H}^{j} \circ D R$. Puisque $d_{r}$ sont des morphismes de $M G_{h}(\mathscr{B}, \mathbb{R}), d_{1}$ est strict, $E_{2}^{p q} \in M H(X, \mathbb{k}, q)^{p}$ et $d_{r}=0$ pour $r \geq 2$ d'après 5.1.14, 5.1.11. Par hypothèse, on a (5.3.5.2) pour $k=1$ D'après $5.1 .10,5.1 .11,5.1 .14$ (et par la décomposition par support strict), les autres assertions sont réduites à:

5.3.6. Lemme. Soient ${ }_{1} C_{i}^{j}$ des $\mathbb{k}$-structures de Hodge de poids $i+j+r$ pour $i, j \in \mathbb{Z}$, telles que ${ }_{1} C_{i}^{j}=0$ si $|i| \gg 0$ ou $|j| \gg 0$. Soient

$$
d_{1}:{ }_{1} C_{i}^{j} \rightarrow{ }_{1} C_{i-1}^{j+1}, N:{ }_{1} C_{i}^{j} \rightarrow{ }_{1} C_{i-2}^{j}(-1), l:{ }_{1} C_{i}^{j} \rightarrow{ }_{1} C_{i}^{j+2}(1)
$$


des morphismes de structures de Hodge, qui commutent mutuellement, et tels que $d_{1}^{2}=0$ et que (5.3.5.2) est satisfaite (pour $k=1$ ). Posons

$$
{ }_{2} C_{i}^{j}=\operatorname{Ker}\left(d_{1}:{ }_{1} C_{i}^{j} \rightarrow{ }_{1} C_{i-1}^{j+1}\right) / \operatorname{Im}\left(d_{1}:{ }_{1} C_{i+1}^{j-1} \rightarrow{ }_{1} C_{i}^{j}\right)
$$

Soient $S: C_{i}^{j} \otimes C_{-i}^{-j} \rightarrow k(-r)$ des accouplements de structures de Hodge tels que

$$
\begin{aligned}
& S(N x \otimes y)+S(x \otimes N y)=0, \quad S(l x \otimes y)=S(x \otimes l y) \\
& S(d x \otimes y)+(-1)^{j} S(x \otimes d y)=0 \quad\left(\text { où } x \in{ }_{1} C_{i}^{j}\right)
\end{aligned}
$$

et que $(-1)^{j(j-1) / 2} S \circ\left(i d \otimes N^{i} l^{j}\right)$ est une polarisation de structures de Hodge sur la partie bi-primitive de ${ }_{1} C_{i}^{-j}$ pour $i, j \geq 0$ (cf. (5.3.5.3)). Alors (5.3.5.2) pour $k=2$ et (5.3.5.3) sont valables pour ${ }_{k} C_{i}^{j}$ définies ci-dessus.

Preuve. Cela résulte de 4.2.2 et de la preuve de 4.1.10, 4.2.2. (On peut aussi utiliser [11, II].) Ce qui achève la démonstration de 5.3.1 (dans le cas $\operatorname{dim} f(Z)>0)$.

5.3.7. Remarque. Lorsqu'on a démontré, 4.2 .2 et 4.2 .4 , les signes n'étaient pas encore déterminés (i.e. 5.2.4 et 5.2.16 n'étaient pas obtenus). Les convensions de signe dans le lemme ci-dessus sont dues à [11, II]. Notons que ce sont compatibles avec (5.2.10.2), (5.3.1.3) et 5.2.16, etc. (et aussi avec un résultat de Schmid).

5.3.8. Preuve de 5.3.1 dans le cas $\operatorname{dim} f(Z)=0$.

D'après 5.1.9 et 5.2.11, on peut supposer que $X=\boldsymbol{P}^{N}, Y=p t$ et $l=c_{1}\left(\Theta_{X}(1)\right)$. (Désormais $Y$ désignera un hyperplan de $X$.) Soit $A$ un sous-espace $\boldsymbol{P}^{N-2}$ de $X$, strictement non-caractéristique pour $(M, F)$, cf. 3.5.1. Soit $\pi: \widetilde{X} \rightarrow X$ l'éclatement le long de $A$, et soit $p: \widetilde{X} \rightarrow P^{1}$ la projection telle que $p^{-1}(t)$ sont les hyperplans contenant $A$. On a alors une immersion fermée $\pi \times p: \widetilde{X} \rightarrow X \times \boldsymbol{P}^{1}$. Posons

$$
\begin{aligned}
& (\tilde{M}, F, \tilde{K})=\pi^{*}(M, F, K)\left(=(\pi \times p)^{*} p r_{1}^{*}(M, F, K)\right) \quad \text { (cf. 3.5.1) } \\
& \tilde{S}=\pi^{*} S: \tilde{K} \otimes \tilde{K} \rightarrow a_{\tilde{X}}^{!} k(-n),
\end{aligned}
$$

alors $\tilde{\boldsymbol{S}}$ est compatible avec la filtration de Hodge (cf. 5.2.10), car $\pi \times p$ est strictement non-caractéristique pour $\operatorname{pr}_{1}^{*}(M, F)$ [1]. D'abord admettons:

$$
\pi_{*}(\tilde{M}, F, \tilde{K})=(M, F, K) \oplus\left(i_{A}\right)_{*} i_{A}^{*}(M, F, K)(-1)[-2]
$$

où $i_{A}: A \rightarrow X$. (La démonstration sera donnée en 5.3.9), Alors $\pi_{*} S$ est compatible avec la decomposition par support strict (5.3.8.1), et la restriction de $\pi_{*} \tilde{S}$ à $K$ coïncide avec $S$, car ils coïncident sur $X \backslash A$. Soit $Y$ un fibre de $p$, 
soient $\tilde{i}_{Y}: Y \rightarrow \tilde{X}$ et $i_{Y}: Y \rightarrow X$ les inclusions naturelles, alors $i_{Y}=\pi \circ \tilde{i}_{Y}$ et donc $i_{Y}^{*}((M, F, K), S) \simeq i_{Y}^{*}((\tilde{M}, F, \tilde{K}), \tilde{S})$. Soit $t$ une coordonnée locale de $\mathbb{P}^{1}$ telle que $Y=p^{-1} t^{-1}(0)$, posons $h=t \circ p$. Soit $g$ une équation locale de $Y$ dans $X$. Puisque $Y$ est strictement non-caractéristique pour $(\tilde{M}, F)$ (resp. $(M, F)$ ) dans un voisinage de $A$, ce Module filtré est quasi-unipotent et régulier le long de $h$ (resp. $g$ ) et on a

$$
\psi_{h}((\tilde{M}, F, \tilde{K}), \tilde{S}) \simeq \tilde{i}_{Y}^{*}((\tilde{M}, F, \tilde{K}), \tilde{S}) \simeq i_{Y}^{*}((M, F, K), S) \underset{\rightarrow}{\rightarrow} \psi_{g}((M, F, K), S)
$$

et $\phi_{h, 1}(\tilde{M}, F, \widetilde{K})=0$ (en particulier $N=0$ et $\psi_{h}=\psi_{h, 1}$ ) au voisinage de $A$ d'après 3.5.6. Alors toutes les hypothèses de 5.3.4 sont satisfaites pour $p: \tilde{X} \rightarrow \mathbb{P}^{1}, \tilde{l}=$ $\pi^{*} l, t, \quad(\tilde{M}, F, \tilde{K})$ et $\tilde{S}$ par hypothèse de récurrence, car $((\tilde{M}, F, \tilde{K}), \tilde{S})$ et ((M,F,K),S) coïncident sur $\widetilde{X} \backslash \pi^{-1}(A)=X \backslash A$. On a donc (5.3.4.1-4) pour toute coordonnée locale de $\mathbb{P}^{1}$; en particulier, $p_{*}(\tilde{M}, F)$ est strict, $\mathcal{H}^{j} p_{*}(\tilde{M}, F, \tilde{K})$ admet la décomposition par support strict d'après 5.2.14 et toutes les hypothèse dans l'assertion suivante (dont la preuve sera donnée en 5.3.10) sont satisfaites pour la $\boldsymbol{P}^{1}$-composante de $P_{\tilde{l}} \mathscr{H}^{-j} p_{*}(\tilde{M}, F, \tilde{K})$ et de ${ }^{p} \mathscr{H} p_{*} \tilde{S} \circ\left(i d \otimes \tilde{l}^{j}\right)$ :

(5.3.8.2) Soient $X$ une variété complexe lisse compacte kählérienne de dimension $1,(M, F, K) \in M F_{h}\left(\mathscr{D}_{X}, k\right)$ à support strict $X$, et $S: K \otimes K \rightarrow$ $a_{X}^{1} k(-n)$ un accouplement. $\mathrm{Si}(M, F)$ est quasi-unipotent et régulier le long de toute coordonnée locale $t$, de sorte que $\operatorname{Gr}_{i}^{W} \psi_{t}(M, F, K)$, $G r_{i}^{W} \phi_{t, 1}(M, F, K) \in M H(p t, k, i)$ (où $W$ est décalée par $n-1$ ou $n$, cf. (5.1.6.2) et que $G r^{W} \psi_{t} S\left(i d \otimes N^{j}\right)$ est une polarisation sur $P_{N} G r_{n-1+j}^{W} \psi_{t}(M, F, K)$ pour $j \geq 0$, alors l'assertion (5.3.1.1) et l'assertion (5.3.1.3) pour $i=0$ sont valables pour $f: X \rightarrow p t$.

(Notons que c'est le unique point où on utilise la théorie de Hodge classique (cf. [36]) dans la preuve de 5.3.1.) D'après [8], 2.1.12 et (5.3.4.3), on a la décomposition non-canonique:

$$
p_{*}(\tilde{M}, F) \simeq \oplus \mathscr{H}^{j} p_{*}(\tilde{M}, F)[-j] \text { dans } D^{b} F\left(\mathscr{D}_{\boldsymbol{P}^{1}}\right),
$$

donc la suite spectrale de Leray pour $a_{\tilde{X}}=a_{P^{10}} p$ dégénère en $E_{2}$ et on a (5.3.4.1) pour $(\tilde{M}, F, \tilde{K})$ et $a_{\tilde{X}}: \tilde{X} \rightarrow p t$ (et de même pour $(M, F, K)$ et $a_{X}: X \rightarrow p t$ d'après (5.3.8.1)), car $M H(p t, k, i)$ est stable par extensions dans $M F_{h}(p t, k)$, cf. [6].

Soit $Y$ un fibre générique de $p$, alors $Y$ est strictement non-caractéristique pour $(M, F)$, donc

$$
\left(M^{\prime}, F, K^{\prime}\right):=i_{Y}^{*}(M, F, K)[-1] \in M H(Y, k, n-1)
$$

et $S^{\prime}:={ }^{p} i \frac{*}{Y} S$ est une polarisation par le même argument que ci-dessus où ${ }^{p} i_{Y}^{*} S$ 
est définie comme ${ }^{p} \psi S$ dans 5.2.3. On a de plus:

$$
\begin{aligned}
& \left(H\left(a_{X}\right)_{*} S\right)\left(u, l^{j} v\right)=(-1)^{j-1}\left(H\left(a_{Y}\right)_{*} S^{\prime}\right)\left(i_{Y}^{*} u, l^{j-1} i_{Y}^{*} v\right) \\
& \text { pour } u, v \in H^{-j}(X, K)\left(=H^{-j}\left(a_{X}\right)_{*} K\right)
\end{aligned}
$$

(La démonstration sera donnée en 5.3.11.) Par hypothèse de récurrence, les assertions (5.3.1.1-3) sont valables pour $a_{Y}: Y \rightarrow p t, l^{\prime}:=i * \frac{*}{Y} l,\left(M^{\prime}, F, K^{\prime}\right)$ et $S^{\prime}$. D'après 3.5.9-10, on a les morphismes compatibles avec les structures de Hodge:

$$
\begin{aligned}
& i_{Y}^{*}: H^{j}(X, K) \rightarrow H^{j+1}\left(Y, K^{\prime}\right) \\
& \left(i_{Y}\right)_{*}: H^{j-1}\left(Y, K^{\prime}\right) \rightarrow H^{j}(X, K)(1) .
\end{aligned}
$$

On a de plus $\left(i_{Y}\right)_{*} i_{Y}^{*}=l: H^{j}(X, K) \rightarrow H^{j+2}(X, K)(1)$, car $\left.K \rightarrow K\right|_{Y \rightarrow \boldsymbol{R}} \Gamma_{Y} K(1)$ [2] $\rightarrow K(1)$ [2] s'identifie au même morphisme pour $K=\boldsymbol{Z}_{X}$, tensorisé par $K$. D'après 2.1 .18 (voir aussi [1]), $i_{Y}^{*}$ (resp. $\left(i_{Y}\right)_{*}$ ) est bijectif pour $j \leq-2$ (resp. $j \geq 2$ ) et injectif pour $j=-1$ (resp. surjectif pour $j=1$ ), donc (5.3.1.2) pour $(M, F, K)$ et pour $j \geq 2$ résulte de la même pour $\left(M^{\prime}, F, K^{\prime}\right)$ et pour $j-1$, de plus on a (5.3.1.3) pour $((M, F, K), S)$ et pour $j \geq 1$ d'après (5.3.8.3) et (5.3.1.3) pour $\left(\left(M^{\prime}, F, K^{\prime}\right), S^{\prime}\right)$, car $i_{Y}^{*}\left(P_{l} H^{-j}(X, K)\right) \subset P_{l} H^{1-j}\left(Y, K^{\prime}\right)$ pour $j \geq 0$ par l'injectivité de $\left(i_{Y}\right)_{*}$ ci-dessus pour $j \geq 2$. D'après (5.3.1.2) pour $j=3$, on a des décompositions:

$$
\begin{aligned}
& H^{-1}(X, K)=l\left(H^{-3}(X, K)(-1)\right) \oplus P_{l} H^{-1}(X, K) \\
& H^{1}(X, K)=l^{2}\left(H^{-3}(X, K)(-2)\right) \oplus \operatorname{Ker} l
\end{aligned}
$$

compatibles avec les structures de Hodge et avec l'action de $l$, alors l'injectivité de

$$
l: P_{l} H^{-1}(X, K) \rightarrow \operatorname{Ker} l
$$

résulte de (5.3.8.3) pour $j=1$ et de la positivité, i.e. de (5.3.1.3) pour $\left(\left(M^{\prime}, F, K^{\prime}\right), S^{\prime}\right)$ et pour $j=0$; d'où (5.3.1.2) pour $((M, F, K), S)$ et pour $j=1$, car $\operatorname{dim} H^{-1}(X, K)=\operatorname{dim} H^{1}(X, K)$ par dualité. Donc il reste à démontrer (5.3.1.3) pour ( $(M, F, K), S)$ et pour $j=0$. On va la réduire à (5.3.8.2) par la suite spectrale de Leray.

Soit $L$ la filtration décroissante de $H^{j}(X, K)$ (compatible avec la structure de Hodge) associée à la suite spectrale de Leray (perverse) ci-dessus, i.e. $G r_{L}^{i} H^{i+j}(\tilde{X}, \tilde{K})=H^{i}\left(\boldsymbol{P}^{1},{ }^{p} \mathcal{H}^{j} p_{*} \tilde{K}\right)$, on a alors

$$
\pi^{*}\left(P_{l} H^{0}(X, K)\right) \subset L^{0} H^{0}(\tilde{X}, \tilde{K})
$$

car 


$$
\begin{aligned}
& L^{0} H^{0}(\tilde{X}, \tilde{K})=\operatorname{Ker}\left(\tilde{i} \tilde{Y}_{Y}^{*}: H^{0}(\tilde{X}, \tilde{K}) \rightarrow H^{1}\left(Y, K^{\prime}\right)\right) \\
& P_{l} H^{0}(X, K)=\operatorname{Ker}\left(i_{Y}^{*}: H^{0}(X, K) \rightarrow H^{1}\left(Y, K^{\prime}\right)\right)
\end{aligned}
$$

pour $Y$ comme ci-dessus, i.e., $Y$ est strictement non-caractéristique pour $(M, F)$. (En fait, $\left({ }^{p} \mathscr{H}^{1} p_{*} \tilde{K}\right)[-1]$ est un système local dans un voisinage du point correspondant à $Y$, dont le fibre à ce point est $H^{1}\left(Y, K^{\prime}\right)$; donc $G r_{L}^{-1} H^{0}(\tilde{X}, \tilde{K})$ est la partie invariante par le groupe de monodromie globale de $H^{1}\left(Y, K^{\prime}\right)$.) De plus

$$
\pi^{*}: P_{l} H^{0}(X, K) \rightarrow G r_{L}^{0} H^{0}(\tilde{X}, \tilde{K})
$$

est injectif et

$$
\pi^{*}\left(P_{l} H^{0}(X, K)\right)\left(\bmod L^{1}\right) \subset H^{0}\left(\mathbb{P}^{1}, P_{\tau}^{p} \mathscr{H}^{0} p_{*} \tilde{K}\right)
$$

où on a la décomposition compatible avec les structures de Hodge:

$$
G r_{L}^{0} H^{0}(\tilde{X}, \tilde{K})=\oplus \tilde{l^{k}}\left(H^{0}\left(\mathbb{P}^{1}, P_{\tilde{l}}^{p} \mathscr{H}^{-2 k} p_{*} \tilde{K}\right)(-k)\right)
$$

par l'action de $\tilde{l}=\pi * l$, car

$$
\begin{aligned}
& \tilde{l}\left(L^{i} H^{i+j}(\tilde{X}, \tilde{K})\right) \subset L^{i} H^{i+j+2}(\tilde{X}, \tilde{K}) \text { pour } i, j \in \mathbb{Z}, \\
& \tilde{l}^{j}: G r_{L}^{i} H^{i-j}(\tilde{X}, \tilde{K}) \stackrel{\sim}{\rightarrow} G r_{L}^{i} H^{i+j}(\tilde{X}, \tilde{K})(j) \text { pour } j \geq 0
\end{aligned}
$$

d'après (5.3.4.3). (En fait, la première assertion résulte du dernier isomorphisme pour $(i, j)=(1,1)$ (et de $L^{1}=G r_{L}^{1}$ ) et la deuxième de cet isomorphisme pour $(i, j)=(0,2)$, car $\pi^{*} \circ l=\tilde{l} \circ \pi^{*}$.) D'après le remarque après (5.3.8.1), on a

$$
\left(H\left(a_{X}\right)_{*} S\right)(u, v)=\left(H\left(a_{\tilde{X}}\right)_{*} \tilde{S}\right)\left(\pi * u, \pi^{*} v\right)
$$

pour $u, v \in H^{0}(X, K)$ et $\pi^{*}$ est un morphisme de structures de Hodge, car $\pi^{*}$ : $H^{0}(X, K) \rightarrow H^{0}(\tilde{X}, \tilde{K})$ est induit aussi par la décomposition canonique (5.3.8.1). (Par définition, $\pi^{*}$ est induit par l'adjonction: $\operatorname{Hom}(\tilde{K}, \tilde{K}) \simeq \operatorname{Hom}\left(K, \pi_{*} \tilde{K}\right)$.) Si de plus $u, v \in P_{l} H^{0}(X, K)$, on a

$$
\left(H\left(a_{\tilde{X}}\right)_{*} \widetilde{S}\right)\left(\pi^{*} u, \pi^{*} v\right)=\left(H\left(a_{P^{1}}\right)_{*}^{p} \mathcal{H} p_{*} \tilde{S}\right)\left(\left[\pi^{*} u\right],\left[\pi^{*} v\right]\right)
$$

où $\left[\pi^{*} u\right]:=\pi^{*} u\left(\bmod L^{1}\right) \in H^{0}\left(\mathbb{P}^{1},{ }^{p} \mathscr{H}^{0} p_{*} \tilde{K}\right)$, car la suite spectrale de Leray perverse est auto-duale d'après 5.2.17-20. Donc d'après 5.3.4 pour $((\tilde{M}, F, \tilde{K})$, $\widetilde{S})$ et $p: \widetilde{X} \rightarrow \mathbb{P}^{1}$, l'assertion (5.3.1.3) pour ((M,F,K),S), $a_{X}: X \rightarrow p t$ et pour $j=0$ est réduite à (5.3.8.2) (appliquée à la $\mathbb{P}^{1}$-composante de $P_{\tilde{l}} \mathcal{H}^{0} p_{*}(\tilde{M}, F, \tilde{K})$ ), car la restriction de ${ }^{p} \mathcal{H} p_{*} \tilde{S}$ aux composantes de $P_{\tilde{l}} \mathcal{H}^{0} p_{*}(\tilde{M}, F, \tilde{K})$ à supports ponctuels est une polarisation d'après (5.3.4.4) et 5.2.14. (Notons que c'est le seul point où la compatibilité de signes est essentielle.) Pour terminer la preuve de 5.3.1 (dans le cas $\operatorname{dim} f(Z)=0$ ), il reste à démontrer (5.3.8.1-3). 
5.3.9. Preuve de (5.3.8.1). D'abord on vérifie que $\pi_{*}(\tilde{M}, F, \tilde{K}) \in M F_{h}\left(\mathscr{D}_{X}, \boldsymbol{k}\right)$ (en particulier, $\pi_{*}(M, F)$ est strict) et qu'on a la décomposition canonique:

$$
\pi_{*}(\tilde{M}, F, \tilde{K})=\left(M_{1}, F, K_{1}\right) \oplus\left(M_{2}, F, K_{2}\right)
$$

telle que $\left(M_{1}, F\right)$ (resp. $\left.\left(M_{2}, F\right)\right)$ est localement isomorphe à $(M, F)$ (resp. $\left.\left(i_{A}\right)_{*} i_{A}^{*}(M, F)(-1)[-2]\right)$; alors $(M, F, K)=\left(M_{1}, F, K_{1}\right)$ est clair, car ils coïncident sur $X \backslash A$.

Soient $Y$ un fibre de $p$ et $i_{Y}, \tilde{i}_{Y}$ comme ci-dessus. Soient $\tilde{A}:=\pi^{-1}(A)$ $\left(=A \times \boldsymbol{P}^{1}\right)$ et $\tilde{i_{\tilde{A}}}: \tilde{A} \rightarrow \tilde{X}, \tilde{i}_{A}: A=\tilde{A} \cap Y \rightarrow \tilde{X}$ les inclusions naturelles. Soit $g$ une équation locale de $Y$, posons $h=g \pi$. Alors $h^{-1}(0)=Y \cup \tilde{A}$, et $Y, \tilde{A}$ et $A=Y \cap \tilde{A}$ sont strictement non-caractéristiques pour $(\tilde{M}, F)$ au voisinage de $\tilde{A}$. Donc d'après 3.5.18, $(\tilde{M}, F)$ est quasi-unipotent et régulier le long de $h$ (au voisinage de $\tilde{A}$ ). Soit $W$ la filtration associée à l'action de $N=t \partial_{t}-\alpha \operatorname{sur} G r_{a}^{V}$, cf. 1.3.9. Posons $\left(M^{\prime}, F\right)=i_{Y}^{*}(M, F)[-1],\left(M^{\prime \prime}, F\right)=i_{A}^{*}(M, F)[-2]$ et $\left(M^{\prime \prime \prime}, F\right)=p r_{1}^{*} i_{A}^{*}$ $(M, F)[-1]$, où $p r_{1}: A \times \boldsymbol{P}^{1} \rightarrow A$. Alors d'après 3.5 .15 on a pour $-1 \leq \alpha \leq 0$ :

$G r_{i}^{W} G r_{a}^{V} i_{h *}(\tilde{M}, F)= \begin{cases}\tilde{i}_{A *}\left(M^{\prime \prime}, F\right)(-1) & \text { si } \alpha=i=-1 \\ \tilde{i}_{Y *}\left(M^{\prime}, F\right)(-1) \oplus \tilde{i}_{\tilde{A} *}\left(M^{\prime \prime \prime}, F\right)(-1) & \text { ou } \alpha=i=0 \\ \tilde{i}_{A *}\left(M^{\prime \prime}, F\right)(-2) & \text { si } \alpha=-1, i=0 \\ 0 & \text { si } \alpha=-1, i=1\end{cases}$

On vérifie directement que $\left(a_{\boldsymbol{P}^{1}}\right)_{*}\left(\Omega_{\boldsymbol{P}^{1}}^{1}, F\right)$ est strict et que

$$
H^{j}\left(a_{\boldsymbol{P}^{1}}\right)_{*}\left(\Omega_{\boldsymbol{P}^{1}}^{1}, F\right)= \begin{cases}(\boldsymbol{C}, F) & \text { si } j=-1 \\ (\boldsymbol{C}, F[-1]) & \text { si } j=1 \\ 0 & \text { sinon }\end{cases}
$$

où $G r_{i}^{F} \Omega_{P^{1}}^{1}=0$ pour $i \neq-1$ et $G r_{i}^{F} C=0$ pour $i \neq 0$. Puisque $\left(M^{\prime \prime \prime}, F\right)=\left(M^{\prime \prime}\right.$, $F) \widehat{\bigotimes}\left(\Omega_{P^{1}}^{1}, F\right)$, on voit que $\left(p r_{1}\right)_{*}\left(M^{\prime \prime \prime}, F\right)$ est strict et que

$$
\mathcal{H}^{j}\left(p r_{1}\right)_{*}\left(M^{\prime \prime \prime}, F\right)= \begin{cases}\left(M^{\prime \prime}, F\right) & \text { si } j=-1 \\ \left(M^{\prime \prime}, F\right)(-1) & \text { si } j=1 \\ 0 & \text { sinon. }\end{cases}
$$

Considéront la suite spectrale pour $\alpha=-1,0$ :

$$
{ }_{\alpha} E_{1}^{-i, i+j}=\mathcal{H}^{j} \pi_{*} G r_{i}^{W} G r_{a}^{V} i_{h *}(\tilde{M}, F) \Rightarrow \mathcal{H}^{j} \pi_{*} G r_{a}^{V} i_{h *}(\tilde{M}, F),
$$

on a alors 


$$
\begin{gathered}
{ }_{-1} E_{1}^{p q}=\left\{\begin{array}{lll}
i_{Y *}\left(M^{\prime}, F\right)(-1) & \text { si }(p, q)=(0,0) \\
i_{A *}\left(M^{\prime \prime}, F\right)(-1) & \text { si }(p, q)=(0,-1),(1,-1) \\
i_{A *}\left(M^{\prime \prime}, F\right)(-2) & \text { si }(p, q)=(0,1),(-1,1) \\
0 & \text { sinon, }
\end{array}\right. \\
{ }_{0} E_{1}^{p q}= \begin{cases}i_{A *}\left(M^{\prime \prime}, F\right)(-1) & \text { si }(p, q)=(0,0) \\
0 & \text { sinon. }\end{cases}
\end{gathered}
$$

Donc $d_{r}=0$ pour $r \geq 2$, i.e. ces suites spectrales dégénèrent en $E_{2}$. On a de plus $\mathcal{H}^{j} \pi_{*} G r_{-1}^{V} i_{h *} \tilde{M}=0$ pour $j \neq 0$, car

$$
\begin{aligned}
D R\left(\mathcal{H}^{j} \pi_{*} G r_{-1}^{V} i_{h *} \tilde{M}\right) & \simeq{ }^{p} \mathcal{H}^{j} \pi_{*} \psi_{h, 1} D R \tilde{M} \quad \text { (d'après 3.4.12) } \\
& \simeq \psi_{g, 1}{ }^{p} \mathcal{H}^{j} \pi_{*} D R \tilde{M}=\psi_{g, 1}{ }^{p} \mathscr{H}^{j}(D R M) .
\end{aligned}
$$

Donc $d_{1}:{ }_{-1} E_{1}^{0,-1} \rightarrow{ }_{-1} E_{1}^{1,-1}$ et $d_{1}:{ }_{-1} E_{1}^{-1,1} \rightarrow{ }_{-1} E_{1}^{0,1}$ sont des isomorphismes en oubliant la filtration. Mais ce sont des isomorphismes filtrés d'après 3.2.2 et 5.1.10 (car un endomorphisme d'un espace vectoriel filtré de dimension finie est strict, s'il est bijectif en oubliant la filtration.) Donc $\pi_{*} G r^{V} i_{h *}(M, F)$ est strict d'après 1.3.5-6, alors d'après 3.3.17 $\pi_{*}(\tilde{M}, F)$ est strict, $\mathcal{H}^{j} \pi_{*}(\tilde{M}, F)$ est quasiunipotent et régulier le long de $g$ et

$$
G r_{a}^{V} i_{g *} \mathcal{H}^{j} \pi_{*}(\tilde{M}, F)=\left\{\begin{array}{lll}
i_{Y *}\left(M^{\prime}, F\right)(-1) & \text { si } & \alpha=-1, j=0 \\
i_{A *}\left(M^{\prime \prime}, F\right)(-1) & \text { si } & \alpha=0, j=0 \\
0 & \text { si } & \alpha \notin \mathbb{Z} \text { ou } j \neq 0,
\end{array}\right.
$$

d'où $\pi_{*}(\tilde{M}, F, \widetilde{K}) \in M F_{h}\left(\mathscr{D}_{X}, \mathbb{R}\right.$ ) et la décomposition (5.3.9.1) d'après 5.1.4. Donc il reste à démontrer que $\left(M_{2}, F, K_{2}\right) \simeq i_{A *} i_{A}^{*}(M, F, K)(-1)[-2]$. Puisque on n'utilise pas ce résultat dans la preuve de 5.3.1, on donne seulement l'esquisse de la preuve. On a le changement de base: $\mathbb{R} \Gamma_{[A]} \pi_{*} \tilde{M}=\pi_{*} \mathbb{R} \Gamma_{[\tilde{A}]} \tilde{M}$ compatible avec $i_{\tilde{A}}^{!} \pi_{*} \tilde{K}=p r_{1 *} i_{A}^{!} \tilde{K}$, par l'image directe de 3.5.9; d'où $\left(M_{2}, K_{2}\right) \simeq i_{A *} i_{A}^{*}(M, K)$ $(-1)$ [-2]. De plus cet isomorphisme est compatible avec $F$ sur un ouvert de Zariski de $A \cap Z$, alors l'assertion résulte de 3.2.2; d'où (5.3.8.1).

5.3.10. Preuve de (5.3.8.2). Soit $j: U \rightarrow X$ un ouvert de Zariski tel que $L=$ $j^{*} K[-1]$ soit un système local, alors $K=\left(j_{*} L\right)[1]$, et $j^{*}((M, F, K), S)$ correspond à une variation de structures de Hodge polarisée de poids $n-1$ comme dans 5.1.10 et 5.2.12. Soit $V$ la filtration de $M$, qui est la filtration de MalgrangeKashiwara (cf. 3.1) à tout point de $X \backslash U$, et telle que $j^{*} V_{a} M=j^{*} M$. D'après (3.2.1.3), on a un quasi-isomorphisme filtré:

$$
a_{X *}(M, F)[-1] \leftarrow a_{X *} C\left(\left(V_{-1} M, F[1]\right) \otimes \omega_{X}^{-1} \rightarrow\left(V_{0} M, F\right)\right)
$$




$$
\left(=\left(K^{\bullet}, F\right) \quad \text { dans }[36,(9.1)]\right)
$$

Donc d'après $[36, \S 7], a_{X} *(M, F, K)$ est un complexe de Hodge de poids $n$; d'où (5.3.1.1) pour $X \rightarrow p t$.

Soit $S^{\prime}: L \otimes L \rightarrow k_{U}(1-n)$ comme dans 5.2.12, alors $S^{\prime}$ est une polarisation au sens de Deligne [6], donc $S^{\prime \prime}=(-2 \pi i)^{n-1} S^{\prime}$ l'est au sens de Griffiths (cf. [36]), i.e.

$$
S^{\prime \prime}\left(C_{x} u, \bar{u}\right) \geq 0 \quad \text { pour } \quad u \in C \otimes L_{x}
$$

où $x \in U$. (Ici, on choisit $i$.) D'après $[36, \S 10(\mathrm{~b})]$, on a

$$
\int_{X} j_{*} S^{\prime \prime}(C u, \bar{u})>0 \quad \text { pour } \quad u \in H^{1}\left(X, \boldsymbol{C} \otimes j_{*} L\right) \text { non nul }
$$

car

$$
j_{*} S^{\prime \prime}: j_{*} L \otimes j_{*} L \rightarrow j_{*}(L \otimes L) \rightarrow j_{*} \boldsymbol{k}_{U}=\boldsymbol{k}_{X}
$$

et

$$
\int_{X} j_{*} S^{\prime \prime}: H^{1}\left(X, j_{*} L\right) \otimes H^{1}\left(X, j_{*} L\right) \stackrel{j_{*} S^{\prime \prime}}{\longrightarrow} H^{2}(X, \boldsymbol{k}) \stackrel{\int_{X}}{\longrightarrow} \boldsymbol{k}
$$

correspondent au produit intérieure $\langle$,$\rangle dans [loc. cit]. Par définition (cf.$ [11], voir aussi la définition de ${ }^{p} \psi$ dans 5.2.3) le diagramme

$$
\begin{aligned}
& H^{1}\left(X, j_{*} L\right) \otimes H^{1}\left(X, j_{*} L\right) \stackrel{j_{*} S^{\prime}}{\rightarrow} H^{2}(X, \boldsymbol{k}(1-n)) \\
& H^{0}(X, K) \otimes H^{0}(X, K) \stackrel{S}{\rightarrow} H^{0}\left(X, a_{X}^{!} \boldsymbol{k}(-n)\right)
\end{aligned}
$$

anti-commute. Puisque $\operatorname{Tr}=(2 \pi i)^{-1} \int_{X}$, on obtient (5.3.1.3) pour $i=0$ et pour $X \rightarrow p t$; d'où (5.3.8.2).

5.3.11. Preuve de (5.3.8.3). Puisque $S \circ(l \otimes i d)=S \circ(i d \otimes l)\left(\right.$ car $\left.l \in H^{2}(X)\right)$, il suffit de vérifier la commutativité du diagramme:

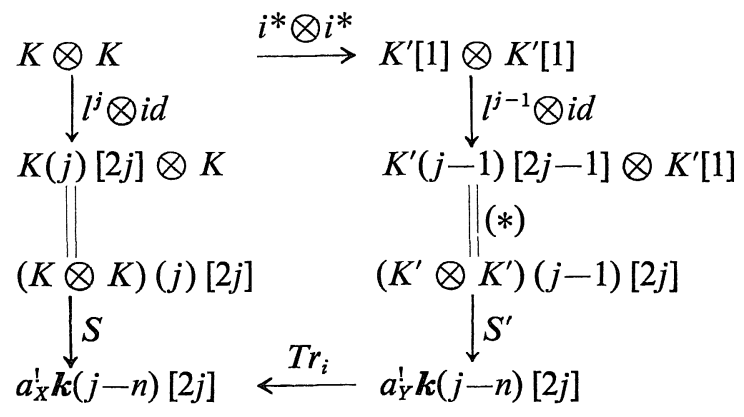


où $i=i_{Y}$, car le signe $(-1)^{j-1}$ résulte de l'isomorphisme $\left(^{*}\right)$ dans le diagramme ci-dessus. (Notons que $K[m]=\mathbb{Z}[m] \otimes K$ par définition [11].) Puisque $i * \circ=$ $l \circ i^{*}$, il suffit de la vérifier pour $j=1$. Par définition de $S^{\prime}$ on a le diagramme commutatif:

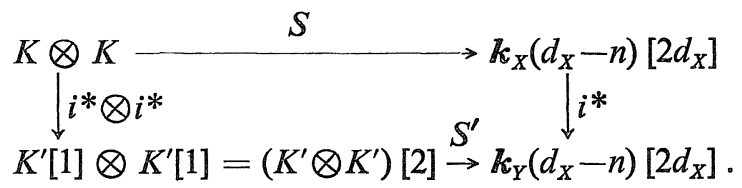

De plus le diagramme

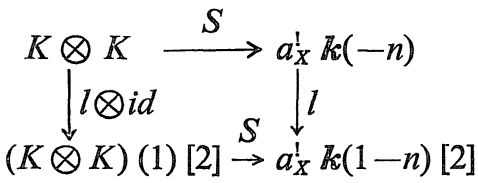

commute, donc l'assertion est réduite à la commutativité de

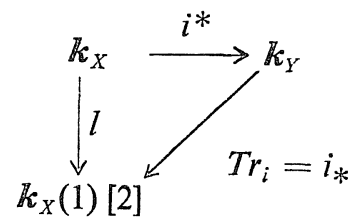

mais c'est la définition (du signe) de $l$; d'où (5.3.8.3). Ce qui achève la démonstration de 5.3.1.

5.3.12. Remarque. Si $X$ n'est pas lisse, on définit $M H(X, \mathbb{k}, n)^{p}$ comme dans 2.1.20, en utilisant $U_{i}$ et $V_{i}$. Alors cette définition est indépendante de $U_{i}, V_{i}$ d'après 5.1.9 et 5.2.11, et $M H_{Z}(X, k, n)^{p}$ sera noté $M H_{Z}(\mathbb{k}, n)^{p}$. De plus 5.3.1 reste valable si $X$ et $Y$ ne sont pas lisses.

\subsection{Variations de structures de Hodge polarisables}

5.4.1. Soit $X$ une variété complexe lisse de dimension $d_{X}$, soit $(L \mathcal{O}, F, L)$ une variation de $\mathbb{k}$-structures de Hodge de poids $n-d_{X}$ (où $L \mathcal{O}=\mathcal{O}_{X} \otimes L$ ), munie d'une polarisation

$$
S^{\prime}: L \otimes L \rightarrow \mathbb{k}\left(d_{X}-n\right) \text {. }
$$

On définit $(M, F, K)$ et $S$ par la réciproque de 5.1 .10 et de 5.2.12, i.e.

$$
\begin{gathered}
(M, F)=\left(\Omega_{X}^{d X}, F\right) \otimes \mathcal{O}(L O, F), \quad K=L\left[d_{X}\right] \\
\mathscr{H}^{-2 d_{X}}(K \otimes K) \stackrel{S}{\longrightarrow} H^{-2 d_{X}}\left(a_{X}^{\prime} \not k(-n)\right) \\
\stackrel{(-1)^{\left.d_{X}{ }^{\left(d_{X}\right.}-1\right) / 2} S^{\prime}}{\longrightarrow} \mathbb{k}\left(d_{X}-n\right)
\end{gathered}
$$


où $G r_{i}^{F} \Omega_{X}^{d_{X}}=0$ pour $i \neq-d_{X}$.

5.4.2. Lemme. S est compatible avec la filtration de Hodge, i.e. $S$ induit la dualité: $(M, F) \underset{\rightarrow}{\sim} \mathbb{D}(M, F)(-n)$

Preuve. Par définition on a

$$
\widetilde{D R}(M, F)\left[-d_{X}\right]=\left[(L \mathcal{O}, F) \rightarrow \Omega_{X}^{1} \otimes(L \mathcal{O}, F[-1]) \rightarrow \cdots \rightarrow \Omega_{X}^{d_{X}} \otimes\left(L \mathcal{O}, F\left[-d_{X}\right]\right)\right]
$$

où le dernier complexe est le complexe de de Rham usuel. Par le produit extérieure, l'accouplement $S$ induit un morphisme de complexes filtrés:

$$
\widetilde{D R}(M, F) \otimes_{C} \widetilde{D R}(M, R)(n) \rightarrow\left(\Omega_{X}^{\cdot}, F\right)\left[2 d_{X}\right]
$$

où $\left(\Omega_{Y}^{\circ}, F\right)=\left(\Omega_{X}^{\circ}, \sigma\right)$, cf. [6]. On vérifie que le morphisme correspondant:

$$
\widetilde{D R}(M, F) \rightarrow \operatorname{Ham}_{c}\left(\widetilde{D R}(M, F),\left(\Omega_{X}^{*}, F\right)\left[2 d_{X}\right]\right)(-n)
$$

induit un morphisme de complexes différentiels filtrés (cf. §2):

$$
\begin{aligned}
\widetilde{D R}(M, F) & \rightarrow \operatorname{Ham}_{\text {Diff }}^{F}\left(\widetilde{D R}(M, F),\left(\Omega_{X}^{\cdot}, F\right)\left[2 d_{X}\right]\right)(-n) \\
& \rightarrow \operatorname{Ham}_{\mathcal{O}}^{F}\left(\widetilde{D R}(M, F),\left(\Omega_{X}^{d_{X}}, F\right)\left[d_{X}\right]\right)(-n) \\
& (\simeq \boldsymbol{D}(\widetilde{D R}(M, F))(-n))
\end{aligned}
$$

et que la composition est un isomorphisme filtré; d'où l'assertion.

5.4.3. Théorème. Soit $((M, F, K), S)$ comme dans 5.4.1, alors c'est un Module de Hodge polarisé de poids $n$.

Preuve. Par récurrence sur $d_{X}$. L'assertion est claire si $d_{X}=0$. En général, il faut vérifier les conditions dans 5.1 .8 et la condition (5.2.10.2) pour tout $g$ (localement définie sur $X$ ). D'abord on ramène au cas où $g^{-1}(0)$ est à croisements normaux.

Soit $f: \tilde{X} \rightarrow X$ une résolution de $g$, i.e. $f^{-1} g^{-1}(0)$ est à croisements normaux, cf. [13]. (Ici, $X$ est locale.) Posons $(\tilde{M}, F, \tilde{K}, \widetilde{S})=f^{*}(M, F, K, S)$, alors cela correspond à la variation de structures de Hodge polarisée $f^{*}\left(L \mathcal{O}, F, L, S^{\prime}\right)$. Si les conditions ci-dessus sont satisfaites pour $g f$ et pour $(\tilde{M}, F, \tilde{K}, \tilde{S})$, on a de même pour $g$ et pour $P_{l} \mathcal{H}^{0} f_{*}(\tilde{M}, F, \tilde{K}, \tilde{S})$ d'après 5.3 .4 et 5.3 .1 , où $l$ est comme dans 5.3.1. De plus $P_{l} \mathcal{H}^{0} f_{*}(\tilde{M}, F, \tilde{K}, \tilde{S})$ admet la décomposition (5.1.4.3) pour la même $g$ d'après 5.2.14. Donc il suffit de vérifier que sa $X$ composante (i.e. $\left(M_{1}, F, K_{1}\right)$ dans (5.1.4.3)) est isomorphe à $(M, F, K)$. Mais on a un morphisme canonique:

$$
f^{*}:(M, K) \rightarrow f_{*}(\tilde{M}, \tilde{K})
$$


qui induit un isomorphisme $(M, K) \simeq\left(M_{1}, K_{1}\right)$ et un isomorphisme filtré $(M, F, K) \simeq\left(M_{1}, F, K_{1}\right)$ en dehors de $g^{-1}(0)$, donc l'assertion résulte de (3.2.2.1) appliqué à $\left(M_{1}, F\right)$, et on peut supposer que $g=\Pi x_{i}^{m}$.

Par le même argument que ci-dessus, on peut de plus supposer que $g=$ $\left(x_{1} \cdots x_{l}\right)^{m}$. En fait, soit $f: \tilde{X} \rightarrow X$ un revêtement ramifié tel que $g f=\left(x_{1} \cdots x_{l}\right)^{m}$, et soient $(\tilde{M}, F, \tilde{K}, \tilde{S})$ et $\left(M_{1}, F, K_{1}\right) \subset \mathcal{H}^{0} f_{*}(\tilde{M}, F, \tilde{K})$ comme ci-dessus, alors on a de même un monomorphisme canonique $f^{*}:(M, K) \rightarrow\left(M_{1}, K_{1}\right)$, qui scinde $\operatorname{par} \mathcal{H}^{0} f_{*} \widetilde{S}$, car elle est une polarisation de variation de structures de Hodge sur $X \backslash g^{-1}(0)$. D'après 3.2.2 ce scindage est compatible avec $F$ et un facteur direct coïncide avec $(M, F, K)$. Donc on peut appliquer 3.6.10 à $(M, F)$ et à $g=\left(x_{1} \cdots x_{l}\right)^{m}$, et on obtient:

(5.4.3.1) $(M, F)$ est quasi-unipotent et régulier le long de $g$,

(5.4.3.2) can: $\psi_{g, 1}(M, F) \rightarrow \phi_{g, 1}(M, F)$ est un épimorphisme strict,

(5.4.3.3) $\quad N^{i}: G r_{n-1+i}^{W} \psi_{g}(M, F) \underset{\rightarrow}{\rightarrow} G r_{n-1-i}^{W} \psi_{g}(M, F)(-i)$ pour $i \geq 0$,

(5.4.3.4) $\quad P_{N} G r_{n-1+i}^{W} \psi_{g}(M, F) \simeq\left(\mathbb{C}^{m}\right) \otimes\left(a_{i+1}\right)_{*} a_{i+1}^{*}(M, F)(-i)[-i-1]$ pour $i \geq 0$ (où $\varepsilon^{i+1}$ se trivialise par l'ordre des coordonnées).

Ici $\mathbb{C}^{m}$ est un espace vectoriel muni de l'action de $T_{s}$ définie par $T_{s} e_{k}=$ $\exp (2 \pi i k / m) e_{k}$, où $\left\{e_{k} ; k \in \mathbb{Z} / m \mathbb{Z}\right\}$ est la base canonique de $\mathbb{C}^{m}$. Notons que l'isomorphisme (5.4.3.4) est compatible avec l'action de $T_{s}$ (où elle est identique sur $\left.\left(a_{i+1}\right) * a_{i+1}^{*}(M, F)\right)$, et qu'il est canonique (à l'action d'une matrice diagonale sur $\boldsymbol{C}^{m}$ près). Donc d'après 5.1.7, il suffit de vérifier qu'il existe un isomorphisme (5.4.3.4) compatible avec la $\mathbb{R}$-structure, tel que l'accouplement $G r^{W} \psi_{g} S \circ\left(i d \otimes N^{i}\right)$ de $P_{N} G r_{n-1+i}^{W} \psi_{g} K$ coïncide, à un constant multiplicatif positif près, à la polarisation induite ${ }^{p} a_{i+1}^{*} S$ de $a_{i+1}^{*}(M, F, K)$ (qui est reliée à $a_{i+1}^{*} S^{\prime}$ comme dans 5.4.1), tensorisé par l'accouplement $S_{m}$ de $\mathbb{Z}^{m}$ définie par la matrice d'identité. (Ici, l'action de $T_{s}$ sur $\mathbb{Z}^{m}$ est définie par $T_{s} e_{i}^{\prime}=e_{i+1}^{\prime}$, où $\left\{e_{i}^{\prime} ; i \in\right.$ $\boldsymbol{Z} / m \boldsymbol{Z}$ \} est la base canonique de $\mathbb{Z}^{m}$.) Mais pour cela on peut supposer que $(M, F, K, S)=\left(\Omega_{X}^{d_{X}}, F, K_{X}, S_{X}\right)$ (donc $\left.n=d_{X}\right)$, cf. 5.2.16, car on a des isomorphismes non-canoniques:

$$
(M, K) \simeq \stackrel{r}{\oplus}\left(\Omega_{X}^{d_{X}}, K_{X}\right), \boldsymbol{C} \otimes(K, S) \simeq \stackrel{r}{\oplus}\left(\mathbb{C} \otimes\left(K_{X}, S_{X}\right)\right)
$$

(En fait, la dernière assertion ne dépend que de $\boldsymbol{C} \otimes(K, S)$.) Alors on peut oublier la filtration $F$, car elle est triviale. Puisque l'ambiguité de (5.4.3.4) ci-dessus est donnée par un endomorphisme du $\mathbb{C}\left[T_{s}\right]$-module $\mathbb{C}^{m}$, il suffit de trouver un isomorphisme: 


$$
P_{N} G r_{n-1+i}^{W} \psi_{g} K \simeq\left(\mathbb{Z}^{m}\right) \otimes\left(a_{i+1}\right)_{*} a_{i+1}^{*} K(-i)[-i-1]
$$

compatible avec l'action de $T_{s}$, tel que la condition de polarisation ci-dessus soit satisfaite. Donc l'assertion résulte de 5.2.16, si $m=1$. Pour $m>1$, on va ramener à ce cas.

Soit $\rho: C \rightarrow C$ un revêtement ramifié tel que $\pi^{*} t=t^{m}$, où $t$ est la coordonnée de $\boldsymbol{C}$. Soit

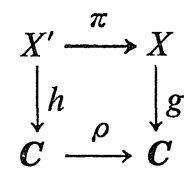

un diagramme cartésien, soit $\sigma: Y \rightarrow X^{\prime}$ la normalisation, posons $U=h^{-1} C^{*}$, $\tilde{\pi}=\pi \sigma, f=h \sigma$, on a alors des isomorphismes canoniques:

$$
{ }^{p} \psi_{g}\left(K_{X}, S_{X}\right) \simeq \pi_{*}^{p} \psi_{h}\left(K_{U}, S_{U}\right) \simeq \widetilde{\pi}_{*}^{p} \psi_{f}\left(K_{Y}, S_{Y}\right)
$$

par définition de ${ }^{p} \psi$; de plus ce sont compatibles avec l'action de $T_{s}$ (où sur les deux derniers elle est induite par un générateur du groupe de transformation) et celle de $N$ (à un constant multiplicatif positif près). Puisque $(Y, f)=$ $\amalg\left(Y_{i}, f_{i}\right)$ et chaque $\left(Y_{i}, f_{i}\right)$ s'identifie à $\left(X, g^{1 / m}\right)$ par $\tilde{\pi}$, on obtient l'assertion. Ce qui achève la démonstration de 5.4.3.

5.4.4. Soient $Z$ une variété analytique complexe irréductible, $U$ un ouvert de Zariski lisse de $Z$, soit $(M, F, K) \in M H(U, \boldsymbol{k}, n)$ muni d'une polarisation $S$. Supposons que $K$ soit lisse, i.e. $L:=K[-\operatorname{dim} U]$ soit un système local, alors $(M, F, K)$ correspond à $(L \mathcal{O}, F, L)$ une variation de $A$-structures de Hodge polarisée d'après 3.2.7 et vice versa d'après 5.4 .3 ; donc on dit que $(M, F, K)$ est une variation de structures de Hodge si $K$ est lisse. Soit $((M, F, K), S)$ une variation de structure de Hodge polarisée, on dit que $((M, F, K), S)$ est semigéométrique rel. à $Z$, s'il existe un morphisme projectif $f: X \rightarrow Z$ tel que $X$ soit lisse, et s'il existe $\left(\left(M^{\prime}, F, K^{\prime}\right), S^{\prime}\right)$ une variation de structures de Hodge polarisée sur $X$ telle que $(M, F, K)$ soit un facteur direct de $\mathscr{H}^{i} f_{*}^{\prime}\left(M^{\prime}, F, K^{\prime}\right)(m)$ pour quelques $i, m \in \mathbb{Z}$ (où $f^{\prime}$ est la restriction de $f$ à $f^{-1}(U)$ ) et que $S$ coïncide avec la restriction de la polarisation induite par $f_{*} S^{\prime}$ et de $l$. (Notons que les assertions (5.3.1.2-3) induisent une polarisation de $\mathcal{H}^{i} f_{*}(M, F, K)$.) On dit que $((M, F, K), S)$ est géométrique rel. à $Z$, si on peut prendre $\boldsymbol{k}_{X}\left[d_{X}\right]$ pour $K^{\prime}$. On dit que $((M, F, K), S)$ est localement (semi-) gêométrique rel. à $Z$, si pour tout $z \in Z$, il existe un voisinage $W$ de $z$ tel que la restriction de $((M, F, K), S)$ 
à $W \cap U$ soit (semi-) géométrique rel à $W$.

5.4.5. Corollaire. Avec les notations de 5.3.12 et 5.4.4, si $((M, F, K), S)$ est localement semi-géométrique rel. à $Z$, il existe uniquement $(\tilde{M}, F, \tilde{K}) \in$ $M H_{Z}(\mathbb{k}, n)^{p}$ avec la polarisation $\tilde{\mathcal{S}}$, dont la restriction d̀ $U$ coïncide à $((M, F, K), S)$.

Preuve. Puisque l'assertion est locale, on peut supposer que $((M, F, K), S)$ est semi-géométrique, alors $((M, F, K), S)$ est un facteur direct de la restriction à $U$ de $\mathcal{H}^{i} f_{*}\left(\left(M^{\prime}, F, K^{\prime}\right), S^{\prime}\right)(m) \in M H(Z, \mathbb{R}, n)^{p}$, cf. 5.3.12. Mais celui-ci se décompose par supports stricts. Soit $\left(\left(M_{Z}, F, K_{Z}\right), S_{Z}\right)$ sa $Z$-composante, alors $((M, F, K), S)$ est un facteur direct de $\left.\left(\left(M_{Z}, F, K_{Z}\right), S_{Z}\right)\right|_{U}$, donc il existe un facteur direct $((\tilde{M}, F, \tilde{K}), \tilde{S})$ de $\left(\left(M_{Z}, F, K_{Z}\right), S_{Z}\right)$, dont la restriction à $U$ coïncide à $((M, F, K), S)$ d'après $3.2 .2-3$ et 5.1 .7 ; d'où l'assertion.

5.4.6. Remarque. Dans le cas algébrique, on dit que $(M, F, K) \in M H_{Z}(\mathbb{k}, n)^{p}$ (polarisé par $S$ ) est (localement) géométrique, si sa restriction à un ouvert lisse non vide de $Z$ soit une variation de structures de Hodge (localement) géométrique. Notons que "localement géométrique" coïncide avec "géométrique" par définition, et qu'on peut éliminer "rel. à $Z$ " d'après Nagata, Hironaka. Alors 5.4.5 entraîne que les Modules de Hodge géométriques sont stables par image directe intermédiaire (et nécessairement polarisables).

5.4.7. Cor@llaire. Soient $Z$ une variété projective irréductible, $U$ un ouvert lisse non vide de $Z$, $(L O, F, L)$ une variation de structures de Hodge géométrique (algébriquement) de poids $n-\operatorname{dim} Z$ sur $U$, alors les cohomologies d'intersection $I H^{i}(Z, L)$ sont munies d'une structure de Hodge canonique polarisable de poids $n+i$, qui ne dépend que de et, est fonctorielle pour, $(L O, F, L)$, ùo $I H^{i}(Z, L)=$ $H^{i}\left(Z, \mathcal{G C}_{Z}(L)\right)$ et $\mathcal{G C}_{Z}(L)=i_{1 *} L[\operatorname{dim} Z]$ avec $i: U \rightarrow Z$. En particulier, les cohomologies d'intersection $\mathrm{IH}^{i}(Z)$ de $Z$ sont munies d'une structures de Hodge canonique polarisable de poids $\operatorname{dim} Z+i$.

(Cela résulte de 5.3.1 et 5.4.5.)

5.4.8. Corollaire. Soient $f: Z \rightarrow Y$ un morphisme projectif de variétés complexes analytiques, $L$ un système local sur un ouvert de Zariski lisse de $Z$, sousjacent à une variation de structures de Hodge polarisée localement semi-géométrique rel. à $Z$; posons $K=\mathcal{G C}_{Z}(L)$, on a alors le théorème de décomposition:

$$
f_{*} K=\oplus\left({ }^{p} \mathcal{H}^{i} f_{*} K\right)[-i] \text { dans } D_{c}^{b}\left(\mathbb{R}_{Y}\right)
$$


(5.4.8.2) ${ }^{p} \mathcal{H}^{i} f_{*} K$ est une somme directe de complexes d'intersection (associés à un système local).

Preuve. Puisque (5.4.8.1) résulte de Lefschetz vache d'après [8], on voit que les assertions sont locales; on peut donc supposer que $Y$ est lisse et que $Z$ est une sous-variété fermée d'une variété complexe lisse $X$, alors 5.4 .8 résulte de 5.3.1 et 5.4.5.

5.4.9. Remarque. Soient $X$ une variété complexe lisse, $Z$ une sous-variété fermée irréductible de $X, U$ un ouvert de Zariski lisse de $Z$, et $(L, F)$ un $\mathscr{D}_{U^{-}}$ Module filtré sous-jacent à une variation de structures de Hodge polarisable. Soient $i: U \rightarrow X^{\prime}:=X \backslash(Z \backslash U)$ et $j: X^{\prime} \rightarrow X$ les inclusions naturelles. Posons $\left(M^{\prime}, F\right)=i_{*}(L, F)$ et $M=j_{1 *} M^{\prime}$, i.e. $M$ est un $\mathscr{D}_{X}$-Module holonome régulier tel que $\left.M\right|_{X^{\prime}} \simeq M^{\prime}$ et que $\mathcal{H}_{X \backslash X^{\prime}}^{0}(M)=\mathcal{H}_{X \backslash X^{\prime}}^{0}(\boldsymbol{D} M)=0$. Soit $M(0)$ le $\mathcal{O}_{X^{-}}$-sousModule de $M$ défini par:

(5.4.9.1) L'ordre modifié au sens de [2,(1.2.1)] est $\leqslant 0$ à tout point lisse de la variété caractéristique de $M$.

Alors Brylinski a défini sa filtration de Hodge:

$$
F_{p}^{\prime} M=\Sigma\left(M(0) \cap j_{*} F_{p-i} M^{\prime}\right)\left(F_{i} \mathscr{D}_{X}\right),
$$

et conjecturé que le théorème de décomposition est valable avec cette filtration de Hodge: pour $f: X \rightarrow Y$ un morphisme projectif et pour $(M, F)$ comme cidessus, $f_{*}(M, F)$ est une somme directe de $\mathscr{D}_{Y}$-Modules filtrés de même type [loc. cit.]. Malheureusement il y a un contre-exemple plutôt trivial à cette conjecture. D'abord on observe:

Si $G r_{p}^{F} L=0$ pour $p \neq 0$, on a $F_{p}^{\prime} M=M(0)\left(F_{p} \mathscr{D}_{X}\right)$

i.e. $G r^{F^{\prime}} M$ est engendré par $G r_{0}^{F^{\prime}} M$ sur $G r^{F} \mathscr{D}_{X}$,

car on a localement $M^{\prime} \simeq L \otimes C[\partial]$ avec $\left.M(0)\right|_{U}=L \otimes 1$. Soient $X=C^{3}, Z=$ $\{P=0\}, U=Z \backslash\{0\}$ avec $P$ un polynôme homogène de degré $d \geqslant 4$. Soit $Y \rightarrow Z$ l'éclatement de 0 , posons $f: Y \rightarrow Z \rightarrow X$, on a alors:

$$
\begin{aligned}
& f_{*}\left(\omega_{Y}, F\right)=(M, F) \oplus\left(\mathscr{B}_{\{0 \mid X}, F[1]\right) \text {, où } M \text { est défini comme ci-dessus } \\
& \text { pour } L=\omega_{U} \text {, mais } G r_{1}^{F} M \neq\left(G r_{0}^{F} M\right)\left(G r_{1}^{F} \mathscr{D}_{X}\right) .
\end{aligned}
$$

On a donc $(M, F) \neq\left(M, F^{\prime}\right)$. Pour éviter ce contre-exemple, on peut ajouter à la fin de (5.4.9.1) la condition "sauf $T_{U}^{*} X$ ". (Mais dans ce cas $M(0)$ n'est plus cohérent sur $\mathcal{O}_{X}$.) Grâce à la difficulté du calcul de l'ordre de KashiwaraKawai [18], je ne connais pas de contre-exemple à cette version modifiée. Mais pour la conjecturer, il y en a trop peu d'évidence (sauf le cas à croisements 
normaux).

5.4.10. Remarque. Dans le cas algébrique, on peut construire la catégorie des Modules de Hodge mixtes géométriques, qui correspond a celle des faisceaux pervers mixtes d'origine géométrique [1] (en particulier, la filtration de monodromie relative existe toujours). Les détails seront donnés dans l'article suivant.

\section{Bibliographie}

[1] A.A. Beilinson, J. Bernstein, P. Deligne, Faisceaux pervers, Astérisque, 100 (1982), 5-171.

[2] J-L. Brylinski, Modules holonomes à singularités régulières et filtration de Hodge II, Astérisque, 101-102 (1983), 75-117.

[3] , - (Co)homologie d'intersection et faisceaux pervers. Séminaire Bourbaki, $n^{\circ}$ 585, Astérisque, 92-93 (1982), 129-157.

[4] J. Cheeger, M. Goresky, R. MacPherson, L2 -cohomology and intersection homology, of singular algebraic varieties, in Seminar on Differential Geometry, Ann. Math. Studies, 102 (1982), 303-340.

[5] H. Clemens, Degeneration of Kähler manifolds, Duke Math. J., 44 (1977), 215-290.

[6] P. Deligne, Théorie de Hodge, I, Actes du Congrès international des Mathématiciens (Nice, 1970), 425-430, II et III, Publ. Math. IHES, 40 (1971), 5-57 et 44 (1974), 5-77.

[7] — La conjecture de Weil II, Publ. Math. IHES, 52 (1980), 137-252.

[8] — Théorème de Lefschetz et critères de dégénérescence de suites spectrales, Publ. Math. IHES, 35 (1968), 107-126.

[9] - Cohomologie à supports propres, SGA4 XVII, Springer Lecture Note, 305 (1973), 250-461.

[10] — Le formalisme des cycles évanescents, SGA7 XIII, Springer Lecture Note, 340 (1973), 82-115.

[11] _ Positivité: signe, I (manuscrit, 16-2-84), II (manuscrit, 6-11-85).

[12] O. Gabber, The integrability of the characteristic variety, Amer. J. Math., 103 (1981), 445-468.

[13] H. Hironaka: Resolution of singularities of an algebraic variety over a field of characteristic zero. I, II, Ann. Math., 79 (1964), 109-203.

[14] - Stratification and flatness, in Real and complex singularities (Proc. Ninth Nordic Summer School/NA VF Sympos. Math., Oslo, 1976), 199-265. Sijthoff and Noordhoff Alphen aan den Rijn, 1977.

[15] C. Houzel, P. Schapira, Images directes des modules différentiels, C.R. Acad. Sci., 298 (1984), 461-464.

[16] M. Kashiwara, On the maximally overdetermined system of linear differential equations, I, Publ. RIMS, Kyoto Univ., 10 (1974/75), 563-579.

[17] — Vanishing cycle sheaves and holonomic systems of differential equations, Springer Lecture Note, 1016 (1983), 134-142.

[18] M. Kashiwara, T. Kawai, On the holonomic systems of micro-differential equations, III, Publ. RIMS, Kyoto Univ., 17 (1981), 813-979.

[19] M. Kashiwara, P. Schapira, Microlocal study of sheaves, Astérisque, 128 (1985), 1 235.

[20] R. Kiehl, J-L. Verdier, Ein einfacher Beweis des Kohärenzsatzes von Grauert, Math. Ann., 195 (1971), 24-50.

[21] G. Laumon, Sur la catégorie dérivée des $\mathscr{D}$-Modules filtrés, Springer Lecture Note, 
1016 (1983), 151-237.

[22] B. Malgrange, Polynôme de Bernstein-Sato et cohomologie évanescente, Astérisque, 101-102 (1983) 243-267.

[23] Z. Mebkhout, Théorème de bidualité pour les $\mathscr{D}_{\boldsymbol{X}}$-Modules bolonomes, Arkiv für Math., 20 (1982), 111-124.

[24] D. Quillen, Higher algebraic K-theory. I, Springer Lecture Note, 341 (1973), 85-147.

[25] J-P. Ramis, G. Ruget, J-L. Verdier, Dualité relative en géométrie analytique complexe, Inv. Math., 13 (1971), 261-283.

[26] M. Saito, Hodge filtration on Gauss-Manin systems. I, J. Fac. Sci., Univ. Tokyo, Sect. I A 30 (1984), 489-498, II, Proc. Japan Acad., Ser. A 59 (1983), 37-40.

[27] — , D-Modules filtrés et structures de Hodge mixtes (manuscrit, oct. 1983).

[28] — Hodge structure via filtered D-Modules, Astérisque, 130 (1985), 342-351.

[29] J.H.M. Steenbrink, Limits of Hodge structures, Inv. Math., 31 (1975/76), 229-257.

[30] — Mixed Hodge structure on the vanishing cohomology, in Rael and complex singularities (Proc. Ninth Nordic Summer School/NAVF Sympos. Math., Oslo, 1976). 525-563. Stijhoff and Noordhoff, Alphen aan den Rijn, 1977.

[31] J-L. Verdier, Catégories dérivées, Etat 0 . in SGA 4 1/2, Springer Lecture Note 569 (1977), 262-308.

[32] — Dualité dans les espaces localement compacts, Séminaire Bourbaki $n^{\circ} \mathbf{3 0 0}$ $(1965 / 66)$.

[33] — Dimension des espaces localement compacts. C.R. Acad. Sci. Paris 261 (1965), 5293-5296.

[34] - Extension of a perverse sheaf over a closed subspace, Asterisque 130 (1985), 210-217.

[35] A.N. Varchenko, The asymptotics of holomorphic forms define a mixed Hodge structure, Soviet. Math. Dokl., 22 (1980), 772-775.

[36] S. Zucker, Hodge theory with degenerating coefficients, $L_{2}$ cohomology in the Poincaré metric, Ann. Math., 109 (1979), 415-476.

[37] H. Cartan, S. Eilenberg, Homological Algebra, Princeton University Press, 1956.

[38] A. Grothendieck, J. Dieudonné, Elément de géométrie algébrique III, Publ. Math. IHES, 11 (1961).

[39] B. Malgrange, Ideals of differentiable functions, Oxford University Press, 1966. 
